\title{
LOW-FLOW FREQUENCY DATA FOR SELECTED STREAM-GAGING STATIONS IN FLORIDA
}

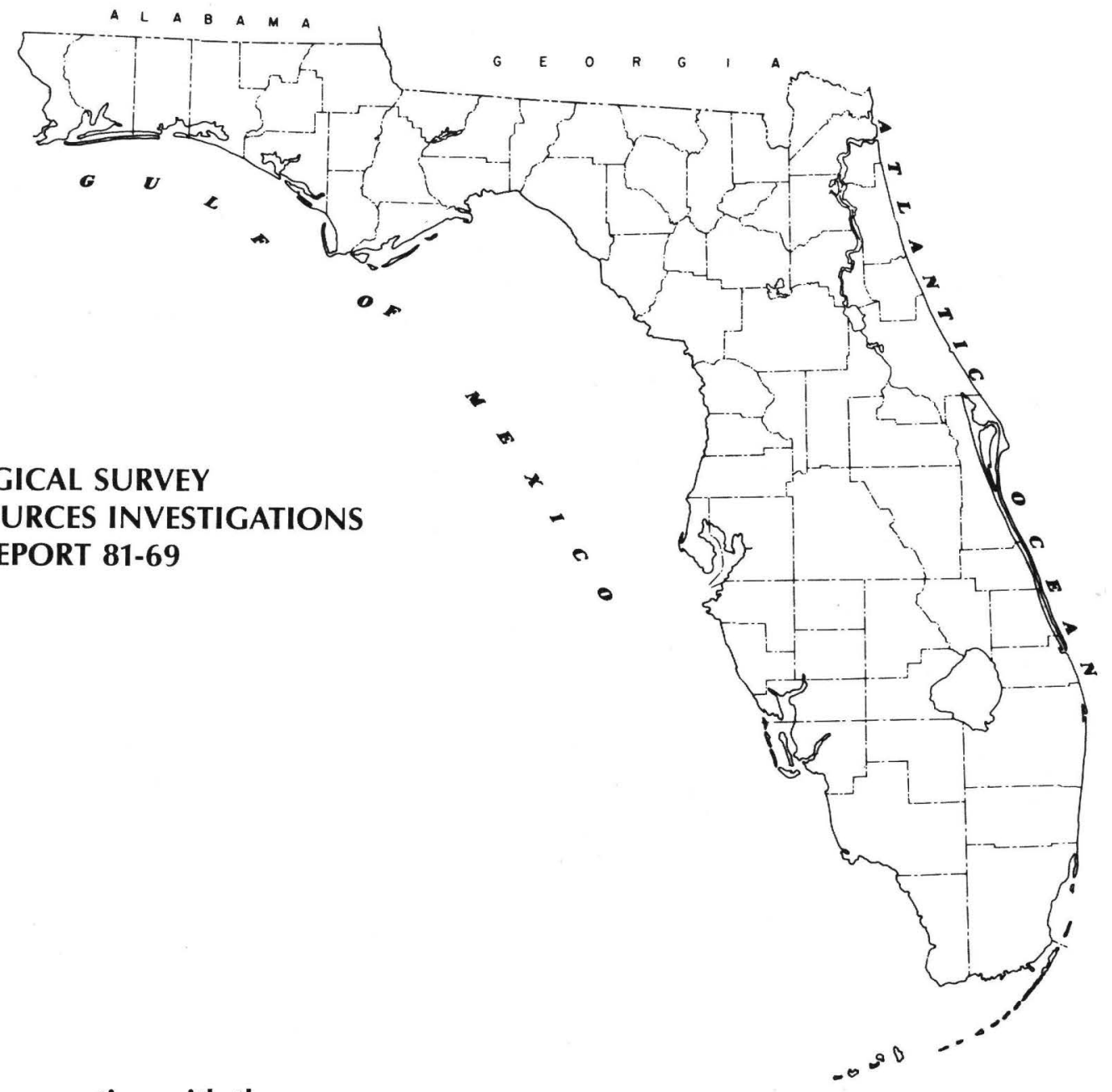

Prepared in cooperation with the

U.S. GEOLOGICAL SURVEY WATER-RESOURCES INVESTIGATIONS OPEN-FILE REPORT 81-69

FLORIDA DEPARTMENT OF ENVIRONMENTAL REGULATION BUREAU OF WATER RESOURCES MANAGEMENT

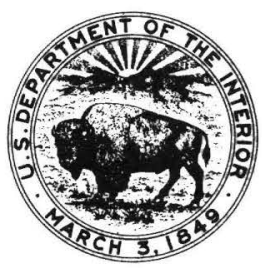




\section{CONVERSION FACTORS}

Factors for converting inch-pound units to International System (SI) units. Multiply inch-pound units

$\underline{B y}$

To obtain SI units

feet (ft)

miles (mi)

square miles $\left(\mathrm{mi}^{2}\right)$

cubic feet per second $\left(\mathrm{ft}^{3} / \mathrm{s}\right)$
0.3048

1.609

2.590

.02832 meters (m)

kilometers $(\mathrm{km})$

kilometers $\left(\mathrm{km}^{2}\right)$

cubic meters per second $\left(\mathrm{m}^{3} / \mathrm{s}\right)$ 


\section{UNITED STATES \\ DEPARTMENT OF THE INTERIOR \\ GEOLOGICAL SURVEY}

LOW-FLOW FREQUENCY DATA FOR SELECTED

STREAM-GAGING STATIONS IN FLORIDA

By G. H. Hughes

U.S. GEOLOGICAL SURVEY

WATER-RESOURCES INVESTIGATIONS

OPEN-FILE REPORT 81-69

Prepared in cooperation with the

FLORIDA DEPARTMENT OF ENVIRONMENTAL REGULATION

BUREAU OF WATER RESOURCES MANAGEMENT

Tallahassee, Florida

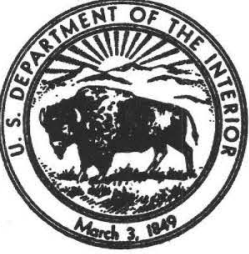

1981 
UNITED STATES DEPARTMENT OF THE INTERIOR

CECIL D. ANDRUS, Secretary

GEOLOGICAL SURVEY

H. William Menard, Director

For additional information write to:

U.S. Geological Survey

325 John Knox Road, F-240

Tallahassee, FL 32303 
Abstract-0.

Introduction--

Methods of analysis-

Frequency curves-

Climatic year-

Extension of frequency curves-

Adjustment of data for short-term stations-

Regulation, diversions, and tidal effects--...

Use of the data--

References

\section{ILLUSTRATIONS}

Figure 1. Location of stream-gaging stations in the St. Marys River basin and the coastal area between the St. Marys and St. Johns Rivers--

2. Location of stream-gaging stations in the St. Johns River basin above the Oklawaha River-...-

3. Location of stream-gaging stations in the Oklawaha River basin--.-.-

4. Location of stream-gaging stations in the St. Johns River basin below the Oklawaha River-...

5. Location of stream-gaging stations in the coastal area between the St. Johns and St. Lucie Rivers-_-

6. Location of stream-gaging stations in the Kissimmee River, Taylor Creek, and Fisheating Creek basins------

7. Location of stream-gaging stations in the Everglades and southeastern coastal area-...

8. Location of stream-gaging stations in the Big Cypress Swamp and southwestern coastal area, including the Caloosahatchee River-..-

9. Location of stream-gaging stations in the Peace and Myakka River basins-_.

10. Location of stream-gaging stations in the Manatee, Little Manatee, Alafia and Hillsborough River basins,

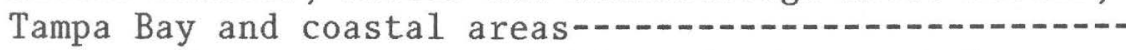

11. Location of stream-gaging stations in the coastal area from Tampa Bay to Withlacoochee River and the Withlacoochee River basin--...-

12. Location of stream-gaging stations in the Waccasassa and Suwannee River basins-1.-.

13. Location of stream-gaging stations in the coastal area between the Suwannee and Aucilla Rivers, and the Aucilla River basin-_...

14. Location of stream-gaging stations in the St. Marks and Ochlockonee River basins and the coastal area between the 0chlockonee and Apalachicola Rivers-.-...-.

15. Location of stream-gaging stations in the Apalachicola River basin including the Chipola River basin-....-.-

16. Location of stream-gaging stations in Choctawhatchee River basin below Pea River, and inflow areas for St. Andrew and Choctawhatchee Bays--

17. Location of stream-gaging stations in the Yellow, Blackwater, Escambia and Perdido River basins-....-.- 
St. Marys River basin

North Prong St. Marys River at Moniac, Ga. 7

Middle Prong St. Marys River at Taylor, Fla.. 7

South Prong St. Marys River:

Turkey Creek at Macclenny, Fla. 8

South Prong St. Marys River at Glen St. Mary, Fla.- 8

St. Marys River near Macclenny, Fla.

Coastal area between St. Marys and St. Johns Rivers

Nassau River:

Thomas Creek near Crawford, Fla.

St. Johns River basin above Oklawaha River

St. Johns River:

Jane Green Creek near Deer Park, Fla. 12

St. Johns River near Melbourne, Fla.

Wolf Creek near Deer Park, Fla.. 13

St. Johns River near Cocoa, Fla. 13

St. Johns River near Christmas, Fla.

Econlockhatchee River:

Little Econlockhatchee River near Union Park, Fla.. 14

Econlockhatchee River near Chuluota, Fla.- 15

Wekiva River near Sanford, Fla.. 15

St. Johns River near De Land, Fla. 16

Oklawaha River basin

Big Creek near Clermont, Fla. 18

Oklawaha River near Ocala, Fla., 18

Silver Springs (head of Silver River) near Ocala, Fla...... 19

Oklawaha River near Conner, Fla.. 19

Oklawaha River at Eureka, Fla. 20

Orange Creek at Orange Springs, Fla. 20

Oklawaha River near Orange Springs, Fla._. 21

Oklawaha River at Riverside Landing, near Orange Springs, Fla.-... 21

St. Johns River basin below Oklawaha River

St. Johns River below Oklawaha River:

Haw Creek:

Little Haw Creek near Seville, Fla.. 23

South Fork Black Creek (head of Black Creek) near Penney Farms,

Fla.

North Fork Black Creek near Middleburg, Fla.- 24

Ortega River at Jacksonville, Fla. 24 
Moultrie Creek at State Highway 207 near St. Augustine, Fla.--.-- 26

Moultrie Creek near St. Augustine, Fla.-_e 26

Tomoka River near Holly Hill, Fla._. 27

Spruce Creek near Samsula, Fla.- 27

Crane Creek at Melbourne, Fla.- 28

Turkey Creek near Palm Bay, Fla.. 28

Fisheating Creek basin

Fisheating Creek near Venus, Fla.. 30

Fisheating Creek at Palmdale, Fla.. 30

Kissimmee River basin

Kissimmee River:

Boggy Creek near Taft, Fla.- 31

Shingle Creek at airport, near Kissimmee, Fla..- 31

Bonnet Creek:
Cypress Creek at Vineland, Fla. 32

Bonnet Creek near Vineland, Fla. 32

Reedy Creek:

Whittenhorse Creek near Vineland, Fla. 33

Reedy Creek near Vineland, Fla.. 33

Davenport Creek near Loughman, Fla.. 34

Catfish Creek near Lake Wales, Fla.- 34

Reedy Creek near Frostproof, Fla..- 35

Arbuckle Creek:
Carter Creek near Sebring, Fla..- 35

Arbuckle Creek near De Soto City, Fla.. 36

Josephine Creek near De Soto City, Fla.. 36

Taylor Creek basin

Taylor Creek near Basinger, Fla. 37

Everglades and southeastern coastal area

Tamiami Canal outlets, levee 67A to 40-mile bend, near Miami, Fla.-- 39

Tamiami Canal outlets, levee 30 to levee 67A, near Miami, Fla.-.-- 39

Big Cypress Swamp and southwestern coastal area

Tamiami Canal outlets, Monroe to Carnestown, Fla.- 41

Tamiami Canal outlets, 40-mile bend to Monroe, Fla.- 41

Barron River Canal near Everglades, Fla._- 42

Faka Union Canal near Copeland, Fla... 42

Henderson Creek Canal near Naples, Fla.. 43

Gordon River:

Golden Gate Canal at Naples, Fla.. 43

Cocohatchee River Canal near Naples Park, Fla._- 44

Imperial River near Bonita Springs, Fla._ 44 
Caloosahatchee River

Page

Caloosahatchee River:

Orange River near Fort Myers, Fla.

Peace River basin

Peace River:

Saddle Creek at structure P-11, near Bartow, Fla.-.

Peace River at Bartow, Fla..

Peace River at Zolfo Springs, Fla. Charlie Creek:

Little Charley Bowlegs Creek near Sebring, Fla.Charlie Creek near Gardner, Fla.-

Peace River at Arcadia, Fla. Joshua Creek at Nocatee, Fla..Horse Creek near Arcadia, Fla..Shell Creek near Punta Gorda, Fla..

Myakka River basin

Myakka River near Sarasota, Fla.

Big Slough near Murdock, Fla..-

Manatee River basin

Manatee River near Myakka Head, Fla.

Manatee River near Bradenton, Fla...

Little Manatee River basin

Little Manatee River near Fort Lonesome, Fla..- 55

Little Manatee River near Wimauma, Fla.

Alafia River basin

North Prong Alafia River at Keysville, Fla.

South Prong Alafia River near Lithia, Fla..--

Little Alafia River near Hopewell, Fla.

Alafia River at Lithia, Fla..

\section{Hillsborough River basin}

Hillsborough River:

Fox Branch near Socrum, Fla.. 58

Blackwater Creek near Knights, Fla. 58

Hillsborough River near Zephyrhills, Fla. 59

New River near Zephyrhills, Fla. 59

Flint Creek near Thonotosassa, Fla. 60

Cypress Creek near San Antonio, Fla. 60

Cypress Creek near Sulphur Springs, Fla.. 61 
Sweetwater Creek near Sulphur Springs, Fla. 62

Rocky Creek near Sulphur Springs, Fla.. 62

Brooker Creek near Odessa, Fla._ 63

Brooker Creek near Tarpon Springs, Fla... 63

Alligator Creek at Safety Harbor, Fla. 64

Coastal area from Tampa Bay to Withlacoochee River

Seminole Lake outlet near Largo, Fla.. 66

Anclote River:

South Branch Anclote River near Odessa, Fla._. 66

Anclote River near Elfers, Fla.. 67

Pithlachascotee River:

Jumping Gully at Loyce, Fla.. 67

Pithlachascotee River near New Port Richey, Fla.. 68

Lighter Bayou:

Bear Creek at Plaza Drive near Hudson, Fla._. 68

Crystal River near Crystal River, Fla.. 69

Withlacoochee River basin

Withlacoochee River near Eva, Fla. 70

Withlacoochee River near Cumpressco, Fla._. 70

Withlacoochee River near Trilby, Fla.. 71

Little Withlacoochee River near Tarrytown, Fla..- 71

Little Withlacoochee River at Rerdell, Fla._. 72

Withlacoochee River at Croom, Fla. 72

Jumper Creek Canal (head of Jumper Creek) near Bushnel1, Fla..... 73

Outlet River at Panacoochee Retreats, Fla.. 73

Withlacoochee River at Wysong Dam, at Carlson, Fla. 74

Withlacoochee River near Holder, Fla. 74

Rainbow Springs (head of Blue Run) near Dunnellon, Fla....... 75

Waccasassa River basin

Waccasassa River near Gulf Hammock, Fla._ 77

Waccasassa River near Otter Creek, Fla. 77

Otter Creek at Otter Creek, Fla. 78

Cow Creek:

Tenmile Creek near Lebanon Station, Fla. 78

Suwannee River basin

Suwannee River at White Springs, Fla. 79

Withlacoochee River near Pinetta, Fla. 79

Suwannee River at Ellaville, Fla.

Suwannee River at Luraville, Fla. 80 
Suwannee River at Branford, Fla. 81

Santa Fe River near Graham, Fla.. 81

Santa Fe River at Worthington Springs, Fla._ 82

Santa Fe River near High Springs, Fla.. 82

Santa Fe River near Fort White, Fla._a 83

Suwannee River near Bell, Fla.. 83

Suwannee River near Wilcox, Fla.. 84

Coastal area between Suwannee and Aucilla Rivers

Steinhatchee River near Cross City, Fla. 86

Fenholloway River near Foley, Fla.. 86

Econfina River near Perry, Fla.. 87

Aucilla River basin

Aucilla River at Lamont, Fla. 88

St. Marks River basin

St. Marks River near Newport, Fla. 90

Ochlockonee River basin

Ochlockonee River:

Sopchoppy River near Sopchoppy, Fla. 91

Ochlockonee River near Havana, Fla. 91

Little River near Quincy, Fla._a

Rocky Comfort Creek near Quincy, Fla.. 92

Telogia Creek near Bristo1, Fla.- 93

Coastal area between Ochlockonee and Apalachicola Rivers

Carrabelle River:

New River near Wilma, Fla. 94

Apalachicola River basin

Apalachicola River at Chattahoochee, Fla. 96

Apalachicola River near Blountstown, Fla.- 96

Chipola River near Altha, Fla.. 97

St. Andrew Bay, inflow area

Econfina Creek near Fountain, Fla.. 99

Econfina Creek near Bennett, Fla.. 99 


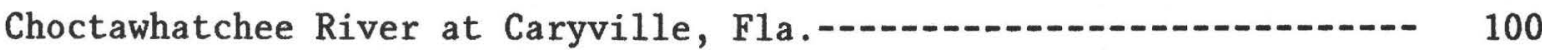

Holmes Creek at Vernon, Fla.-- 100

Choctawhatchee River near Bruce, Fla.-- 101

Choctawhatchee Bay, inflow area

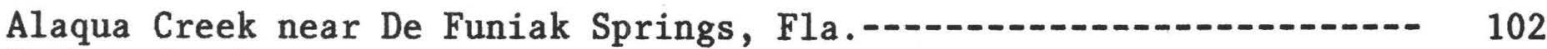
Turkey Creek:

Juniper Creek at State Highway 85, near Niceville, Fla.-.-.-.-- 102

Yellow River basin

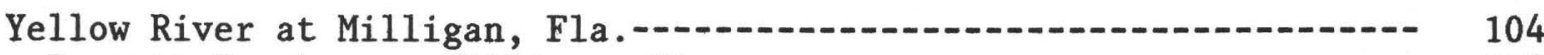

Baggett Creek near Milligan, Fla.-_-_- 104

Shoal River near Mossy Head, Fla.-_- 105

Shoal River near Crestview, Fla.-_- 105

Yellow River near Holt, Fla.- 106

Blackwater River basin

Blackwater River near Baker, Fla.--_-_-_-- 107

Big Juniper Creek near Munson, Fla.-_-_.-.-. 107

Big Coldwater Creek near Milton, Fla.-_- 108

Pond Creek near Milton, Fla.- 108

Escambia River basin

Escambia River near Century, Fla.--_-_-- 109

Pine Barren Creek near Barth, Fla.- 109

Perdido River basin

Perdido River:

Brushy Creek near Walnut Hill, Fla.-_-_.-- 110

Perdido River at Barrineau Park, Fla.-- 110 

LOW-FLOW FREQUENCY DATA FOR SELECTED

STREAM-GAGING STATIONS IN FLORIDA

By G. H. Hughes

\section{ABSTRACT}

This report contains tables of low-flow frequency data for 161 stream-gaging stations in Florida. The selected stations represent those having suitable flow records spanning 7 or more years. Flow values representing the annual minimum average flow for selected periods ranging from 1 to 183 days are presented for recurrence intervals ranging from 2 to 50 years. The low-flow frequency data of relatively short-term stations were adjusted where possible on basis of records of long-term stations to reduce the possible bias introduced by short-term climatic trends.

\section{INTRODUCTION}

Estimates of the probable magnitude and frequency of future low streamflows are required for many uses. Among these are appraisals of the adequacy of streamflow for dilution and transport of wastes and as a source of water for municipal, industrial, and agricultural uses, especially during critical dry periods. Low-flow frequency data are also useful in the administration of water laws.

This report represents an initial effort to compile statewide low-flow frequency data for streams in Florida. The report presents low-flow frequency data for 161 stream-gaging stations which have continuous records spanning 7 years or more as of 1977 . Flow values representing the annual minimum average flow for various time periods are presented in tabular form for recurrence intervals of 2, 5, 10, 20, 30, and 50 years. The recurrence interval is the average number of years between occurrences of annual low flows equal to or less than a specified magnitude.

The scope of this report does not encompass efforts to provide estimates of recurrence intervals of low flows at partial record stations or at stations having less than 7 years continuous record, or to extrapolate low-flow frequency data of gaged sites to ungaged sites, or to establish relations between low streamflows and physiographic factors or features that influence streamflow. A treatment of these topics would require a much more comprehensive study than is provided by this report. 
The procedures of frequency analysis used in this report are basically those described by Riggs (1972). Criteria used by Carter and Putnam (1978) were adopted herein for the extension of frequency curves and for adjustment of low-flow frequency data of relatively short-term stations.

\section{Frequency Curves}

In this report frequency analyses were made of the annual minimum average flow for periods of $1,7,14,30,60,90,120$, and 183 days. The computation of annual low flows and related frequency analyses were done by digital computer using records in the U.S. Geological Survey files in Reston, Va.

The computer output included the annual minimum average flow and its corresponding ranking order number for each time period of concern and each year of record. The computer program provided for a mathematical fit of the logarithms of the annual flow data to curves representing the Pearson Type III frequency distribution. Results of the frequency analyses were obtained in both tables and graphs. In the graphs, the annual flow data were plotted against the apparent recurrence interval which is computed as the ratio: $N+1 / M$, where $N$ is the number of annual flow values and $M$ is the order number.

The computer generated log-Pearson frequency curve provided a satisfactory fit of the annual flow data for many stations, primarily those having long periods of record, but in many other instances provided a poor fit of the data, particularly in the lower range of flows . Consequently, in accordance with the suggestion by Riggs (1972, p. 6), a graphical interpretation of low-flow data was used in instances where the log-Pearson Type III curve appeared to be unsatisfactory.

\section{Climatic Year}

Because the seasonal distribution of rainfall in north Florida differs appreciably from that in the rest of the State, different climatic years were used in the determination of the annual minimum average flows of streams in different parts of the State. The 12-month period ending March 31 was used for streams in north Florida and the 12-month period ending June 30 was used for the rest of the State. The climatic year used for each station is evident from the period of record analyzed as given in the station description.

\section{Extension of Frequency Curves}

The criteria used by Carter and Putnam (1978, p. 13) for the extension of frequency curves was followed in this report. Frequency curves for stations having 7 to 9 years record were extended to the 10-year recurrence interval; those for stations having 10 to 19 years record were extended to the 20-year recurrence interval; those for stations having 20 to 29 years were extended to the 30-year recurrence interval; and those for stations having 30 or more years record were extended to the 50-year recurrence interval. 


\section{Adjustment of Data for Short-Term Stations}

The low-flow frequency data of relatively short-term stream-gaging stations were adjusted where possible to minimize the chance of bias stemming from short-term climatic trends. This was accomplished by use of regression methods wherein the annual 7-day minimum flows of neighboring long- and short-term stations were correlated. Criteria for adjusting the frequency data of short-term stations in this report was adopted from Carter and Putnam (1978, p. 15). These were: (1) that the correlation coefficient of the regression must be 0.8 or greater; (2) that the length of record of the long-term station must be at least twice that of the short-term station; and, (3) that the length of the concurrent flow record must be at least 9 years.

For those stations that met the criteria, the entire array of frequency data of the short-term station was estimated on the basis of the regression equation and the low-flow frequency data of the long-term station. However, the extension of the frequency curve of the short-term station, which as previously explained was varied on the basis of the number of years of available record, remained unchanged. In other words, the frequency curve of a short-term station having 10 to 19 years of continuous record, for example, was extended only to the 20-year recurrence interval even though the frequency data may have been adjusted on the basis of the record of a station having a considerably longer record. Stations for which adjustments were made are noted in the "Remarks" paragraph of the station description.

\section{Regulation, Diversions, and Tidal Effects}

Many of the streams in Florida are to some extent affected by regulation and diversions. Low-flow frequency data were included in this report for gaging stations on such streams if the degree of regulation or diversion appeared to be relatively consistent throughout the period of record analyzed or was of relatively minor importance.

At some of the gaging stations in Florida the flow occasionally reverses as a result of tide and wind effect. In the analysis of the low-flow data for these stations, negative discharges (representing flow in the upstream direction) were treated as zero flow. Stations at which the low-flow data are affected by regulation, diversions, or tide and wind effects are noted in the "Remarks" paragraph of the station description.

\section{USE OF THE DATA}

The low-flow data that follow are applicable with certainty only to the flow at the gaging stations represented. The data for those sites that are subject to appreciable regulation probably are useful only at the sites for which they were determined, and only so long as the pattern and extent of the regulation remains similar to that for period of analysis. The extent to which the data for unregulated streams can be applied to ungaged sites on the same or neighboring streams depends largely on the uniformity of the geologic and meteorologic conditions in the area of 
concern. If conditions are basically the same within an area, the low-flow frequency data for gaged sites sometimes bear a consistent relation to the size of drainage area, and hence, can be related to the flow at ungaged sites by use of drainage areas.

Unfortunately, according to Riggs (1972, p. 11), "Geologic homogeneity with respect to base flow usually cannot be identified from field or geologic-map examination." Furthermore, the "...evapotranspiration loss, which may be a significant factor, is not easily described by an index." Given the complex interrelation between ground and surface water in many parts of Florida, therefore, attempts to estimate low-flow frequencies for ungaged sites involve considerable risk of large errors unless the hydrology of the area is generally defined and at least a few measurements of base flow are obtained at the ungaged site.

\section{REFERENCES}

Carter, R. F., and Putnam, S. A., 1978, Low-flow frequency of Georgia streams: U.S. Geological Survey, Water-Resources Investigations Open-File Report 77-127, 104 p.

Riggs, H. C., 1972, Low-flow investigations: Techniques of water-resources investigations of the United States Geological Survey, Chapter B-1, Book 4, 18 p. 
CONTINUOUS-RECORD STREAM-GAGING STATIONS FOR WHICH

LOW-FLOW FREQUENCY DATA ARE INCLUDED 


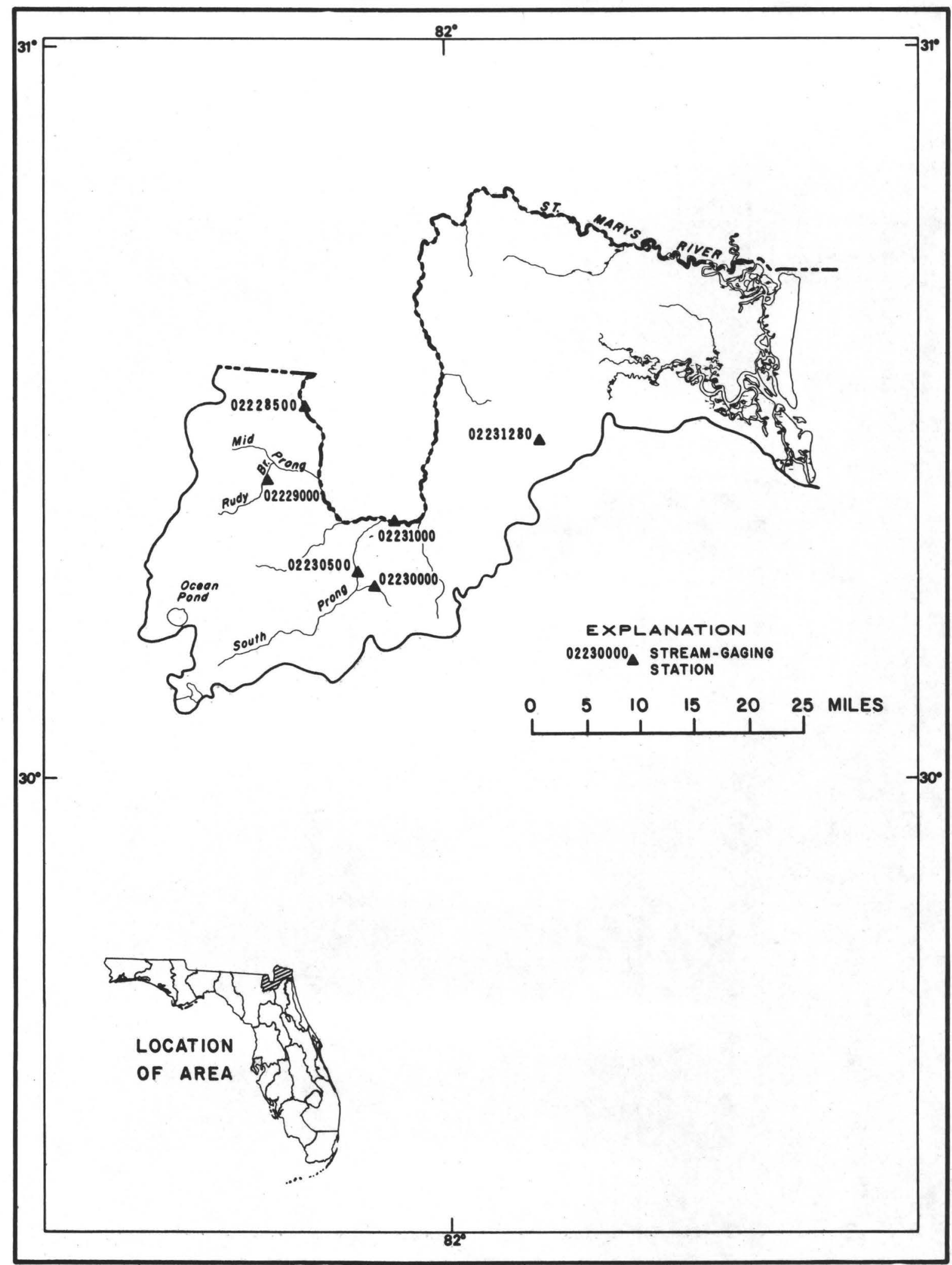

Figure 1.--Location of stream-gaging stations in the St. Marys River basin and the coastal area between the St. Marys and St. Johns River. 
LOCATION.--Lat $30^{\circ} 31^{\prime} 03^{\prime \prime}$, long $82^{\circ} 13^{\prime} 50^{\prime \prime}$, in NW3 sec.8, T.1 N., R.21 E., Baker County, Fla., Hydrologic Unit 03070204, near right bank at upstream side of bridge on State Highways 2 and $94,0.2$ mi upstream from Georgia Southern \& Florida Railway bridge, $0.4 \mathrm{mi}$ west of Moniac, $1.0 \mathrm{mi}$ downstream from Moccasin Creek, and $122 \mathrm{mi}$ upstream from mouth of St. Marys River.

DRAINAGE AREA.--160 $\mathrm{mi}^{2}$, approximately, includes part of watershed in Okefenokee Swamp which is indeterminate.

PERIOD OF RECORD ANALYZED.--April 1921 to March 1923, April 1927 to March 1930, April 1933 to March 1934 , April 1951 to March 1977.

\begin{tabular}{|c|c|c|c|c|c|c|c|c|}
\hline $\begin{array}{l}\text { Recurrence } \\
\text { interval }\end{array}$ & Lلأ & $\begin{array}{l}\text { est av } \\
\text { for in }\end{array}$ & cage & $\begin{array}{l}\text { ow, in } \\
\text { number }\end{array}$ & $\begin{array}{l}\text { cubic } f \\
\text { of cons }\end{array}$ & $\begin{array}{l}\text { eet per } \\
\text { ecutive }\end{array}$ & $\begin{array}{l}\text { secono } \\
\text { days }\end{array}$ & \\
\hline in years & 1 & 7 & 14 & 30 & 60 & 90 & $120^{\circ}$ & 183 \\
\hline 2 & 0.20 & 0.48 & 1.0 & 2.5 & 5.8 & 15 & 29 & 65 \\
\hline 5 & 0 & 0 & 0 & .10 & .60 & 1.5 & 3.9 & 15 \\
\hline 10 & 0 & 0 & 0 & 0 & .15 & .26 & .97 & 8.0 \\
\hline 20 & 0 & 0 & 0 & 0 & $(*)$ & .10 & .25 & .30 \\
\hline 30 & 0 & 0 & 0 & 0 & 0 & 0 & (*) & (*) \\
\hline 50 & 0 & 0 & 0 & 0 & 0 & 0 & 0 & 0 \\
\hline
\end{tabular}

02229000 Middle Prong St. Marys River at Taylor, Fla.

LOCATION.--Lat $30^{\circ} 26^{\prime} 10^{\prime \prime}$, Iong $82^{\circ} 17^{\prime} 15^{\prime \prime}$, in SW sec.2, T.1 S., R.20 E., Baker County, Hydrologic Unit 03070204, near center of span on State Highway 125, $0.5 \mathrm{mi}$ southeast of Taylor, $0.9 \mathrm{mi}$ upstream from Little River, and 7.4 mi upstream from mouth.

DRAINAGE AREA. $--125 \mathrm{mi}^{2}$, approximately .

PERIOD OF RECORD ANALYZED.--April 1956 to March 1967, April 1976 to March 1977.

Recurrence
interval
in years
2
5
10
20

MAGNITUDE AND FREQUENCY OF ANNUAL LOW FLOWS

Lowest average flow, in cubic feet per second, for indicated number of consecutive days

$\begin{array}{llllllll}1 & 7 & 14 & 30 & 60 & 90 & 120 & 183\end{array}$

$\begin{array}{llllllll}1.2 & 1.4 & 1.6 & 2.3 & 4.9 & 11 & 18 & 37\end{array}$

* Less than $0.05 \mathrm{ft}^{3} / \mathrm{s}$. 
LOCATION.--Lat $30^{\circ} 16^{\prime}, 08^{\prime \prime}$, long $82^{\circ} 07^{\prime} 21^{\prime \prime}$, in NE光 sec.5, T.3 S., R.22 E., Baker County, Hydrologic Unit 03070204, near left bank at downstream side of bridge on State Highway $121,0.9 \mathrm{mi}$ south of Macclenny and $1.8 \mathrm{mi}$ upstream from mouth.

DRAINAGE AREA. $--20.9 \mathrm{sq}$ mi.

PERIOD OF RECORD ANALYZED.--April 1956 to March 1969.

\begin{tabular}{|c|c|c|c|c|c|c|c|c|}
\hline $\begin{array}{l}\text { Recurrence } \\
\text { interval }\end{array}$ & MAGNITUDE & $\begin{array}{l}\text { D FREQL } \\
\text { west at } \\
\text { for ir }\end{array}$ & $\begin{array}{l}\text { JCY OF } \\
\text { cage fl } \\
\text { icated }\end{array}$ & $\begin{array}{l}\text { ANNUAL } \\
\text { ow, in } \\
\text { number }\end{array}$ & $\begin{array}{l}\text { LOW FLO } \\
\text { cubic } \\
\text { of cons }\end{array}$ & $\begin{array}{l}\text { WS } \\
\text { eet p } \\
\text { ecuti }\end{array}$ & $\begin{array}{l}r \text { secon } \\
\text { e days }\end{array}$ & \\
\hline in years & 1 & 7 & 14 & 30 & 60 & 90 & $120^{\circ}$ & 183 \\
\hline 2 & 0.51 & 0.61 & 0.79 & 1.1 & 1.7 & 3.0 & 5.3 & 15 \\
\hline 5 & .28 & .39 & .53 & .66 & 1.1 & 1.9 & 2.6 & 7.0 \\
\hline 10 & .15 & .31 & .38 & .52 & .94 & 1.5 & 1.8 & 4.2 \\
\hline 20 & .07 & .23 & .28 & .42 & .79 & 1.3 & 1.4 & 2.6 \\
\hline
\end{tabular}

02230500 South Prong St. Marys River at Glen St. Mary, Fla.

LOCATION.--Lat $30^{\circ} 16^{\prime} 43^{\prime \prime}$, long $82^{\circ} 08^{\prime} 40^{\prime \prime}$, in SWz sec.31, T.2 S., R.22 E., Baker County, Hydrologic Unit 03070204, on right bank $65 \mathrm{ft}$ upstream from bridge on U.S. Highway $90,1.0$ mi east of Glen St. Mary, and 8.2 mi upstream from mouth.

DRAINAGE AREA. $--130 \mathrm{sq}$ mi approximately.

PERIOD OF RECORD ANALYZED.--April 1950 to March 1971.

Recurrence
interval
in years
2
5
10
20
30

\begin{tabular}{|c|c|c|c|c|c|c|c|}
\hline MAGNITUDE & $\begin{array}{c}\text { AND FREC } \\
\text { Lowest } \\
\text { for }\end{array}$ & $\begin{array}{l}\text { QUENCY OF } \\
\text { average } f \\
\text { indicated }\end{array}$ & $\begin{array}{l}\text { ANNUAL } \\
\text { low, in } \\
\text { number }\end{array}$ & $\begin{array}{l}\text { LOW FI } \\
\text { cubic } \\
\text { of cor }\end{array}$ & $\begin{array}{l}\text { OWS } \\
\text { feet pe } \\
\text { secutiv }\end{array}$ & $\begin{array}{l}\text { er secon } \\
\text { ve days }\end{array}$ & \\
\hline 1 & 7 & 14 & 30 & 60 & 90 & $120^{\circ}$ & 183 \\
\hline 2.9 & 3.1 & 3.6 & 4.6 & 7.7 & 13 & 24 & 60 \\
\hline 1.8 & 2.1 & 2.4 & 2.9 & 4.3 & 5.9 & 9.6 & 20 \\
\hline 1.3 & 1.8 & 2.0 & 2.3 & 2.9 & 4.1 & 6.0 & 10 \\
\hline 1.0 & 1.5 & 1.6 & 1.9 & 2.2 & 3.1 & 4.1 & 5.8 \\
\hline .90 & 1.3 & 1.4 & 1.6 & 1.8 & 2.5 & 3.0 & 4.2 \\
\hline
\end{tabular}


02231000 St. Marys River near Macclenny, Fla.

LOCATION.--Lat $30^{\circ} 21^{\prime} 31^{\prime \prime}$, long $82^{\circ} 04^{\prime} 54^{\prime \prime}$, in NW sec.2, T.2 S., R.22 E., Baker County, Hydrologic Unit 03070204, on right bank $200 \mathrm{ft}$ downstream from site of former Stokes Bridge, 1 mi downstream from confluence of North and South Prongs, 6 mi northeast of Macclenny, and $1^{\prime}$ mi upstream from mouth.

DRAINAGE AREA.--700 $\mathrm{mi}^{2}$, approximately, includes part of watershed in Okefenokee Swamp which is indeterminate.

PERIOD OF RECORD ANALYZED.--April 1927 to March 1978.

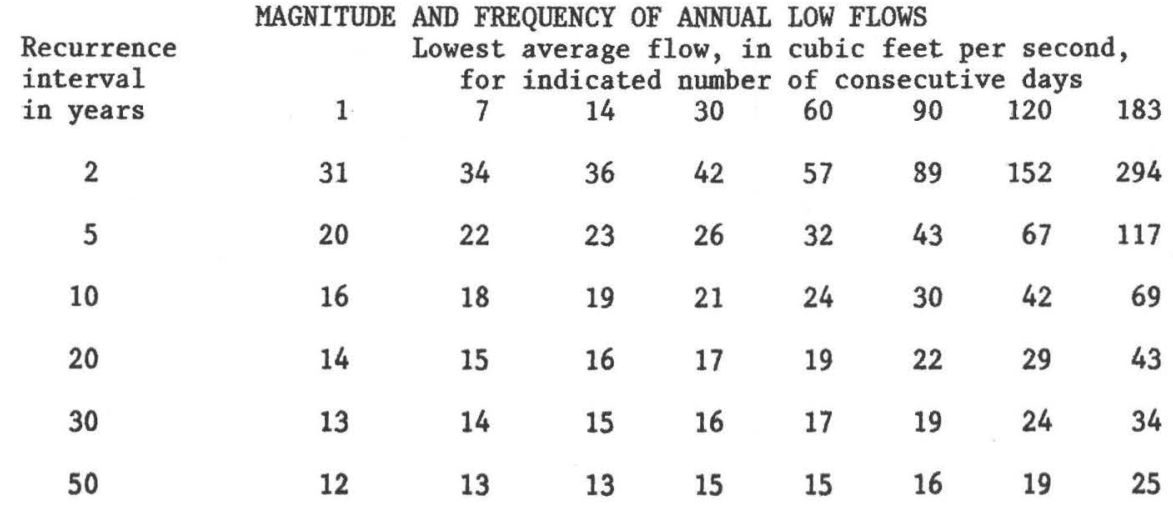


COASTAL AREA BETWEEN ST. MARYS AND ST. JOHNS RIVERS

02231280 Thomas Creek near Crawford, Fla.

LOCATION.--Lat $30^{\circ} 27^{\prime} 39^{\prime \prime}$, long $81^{\circ} 49^{\prime} 57^{\prime \prime}$, in NW $\frac{1}{4}$ sec.32, T.1 N., R.25 E., Duval County, Hydrologic Unit 03070205, on downstream side of bridge on Acree Road, $4.4 \mathrm{mi}$ southeast of Crawford, 4.4 mi northwest of Dinsmore, $7.1 \mathrm{mi}$ south of Callahan, and $24 \mathrm{mi}$ upstream from mouth.

DRAINAGE AREA. $--29.9 \mathrm{mi}^{2}$.

PERIOD OF RECORD ANALYZED.--April 1965 to March 1977.

Recurrence
interval
in years
2
5
10
20

\section{MAGNITUDE AND FREQUENCY OF ANNUAL LOW FLOWS}

Lowest average flow, in cubic feet per second, for indicated number of consecutive days

$\begin{array}{llllllll}1 & 7 & 14 & 30 & 60 & 90 & 120 & 183\end{array}$

$\begin{array}{rrrrrrrr}1.9 & 2.1 & 2.6 & 3.3 & 4.5 & 8.0 & 11 & 17 \\ 1.2 & 1.4 & 1.6 & 2.3 & 3.3 & 5.3 & 7.4 & 11 \\ .90 & 1.1 & 1.2 & 1.9 & 2.6 & 4.3 & 6.2 & 9.0 \\ .70 & .90 & 1.0 & 1.6 & 2.2 & 3.6 & 5.3 & 7.5\end{array}$




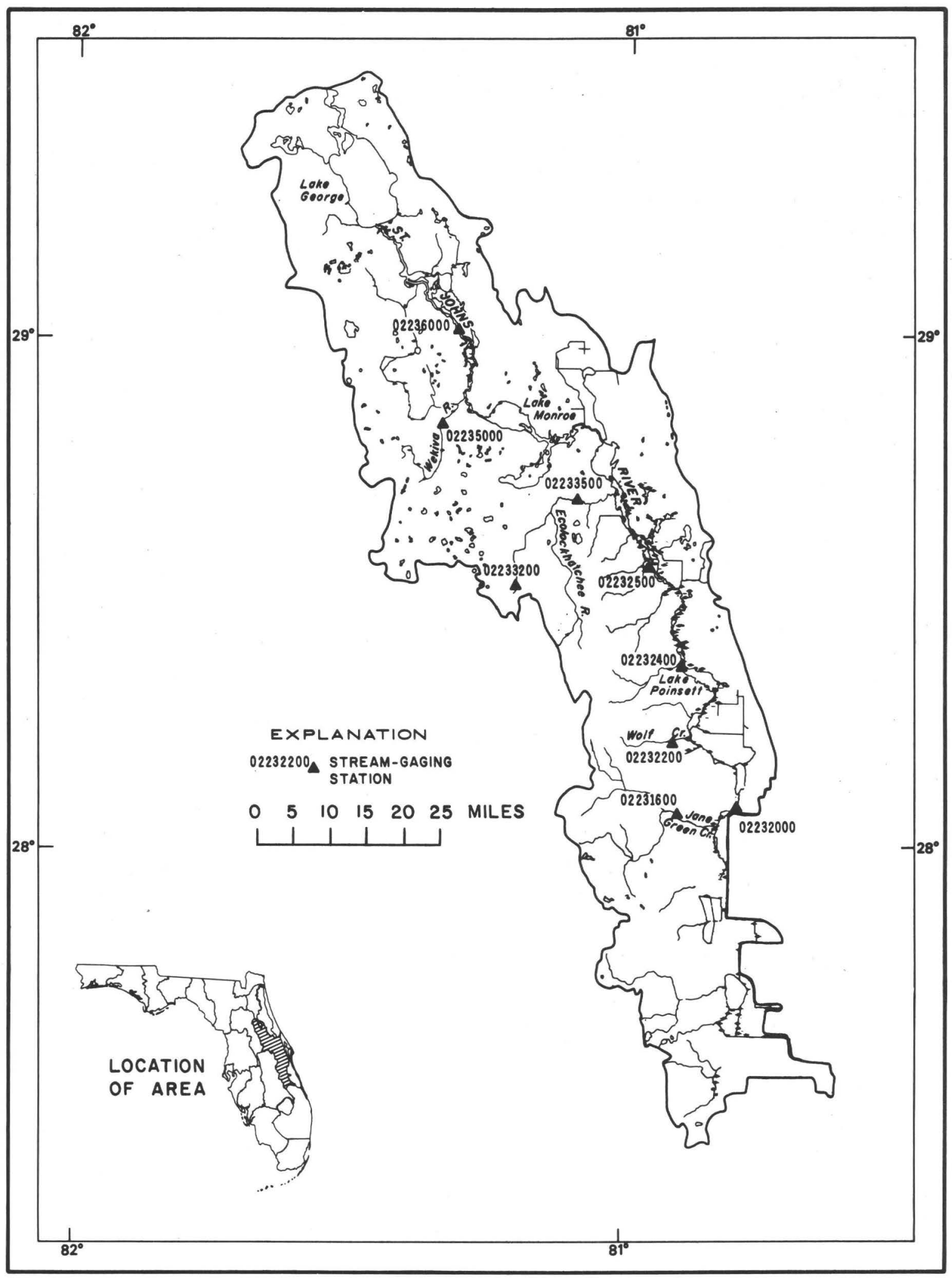

Figure 2.--Location of stream-gaging stations in the St. Johns River basin above the Oklawaha River. 
ST. JOHNS RIVER

ST. JOHNS RIVER BASIN ABOVE OKLAWAHA RIVER

02231600 Jane Green Creek near Deer Park, Fla.

LOCATION.--Lat $28^{\circ} 04^{\prime} 27^{\prime \prime}$, long $80^{\circ} 53^{\prime} 18^{\prime \prime}$, in SE⿺ sec.2, T.28 S., R.34 E., Osceola County, Hydrolgic Unit 03080101, near right bank of leftmost of five channels on upstream side of bridge on county road, $1.2 \mathrm{mi}$ southeast of Deer Park, 2 mi downstream from confluence of Crabgrass and Bull Creeks, and 5.8 mi upstream from mouth.

DRAINAGE AREA. $--248 \mathrm{mi}^{2}$.

PERIOD OF RECORD ANALYZED.--July 1954 to June 1977.

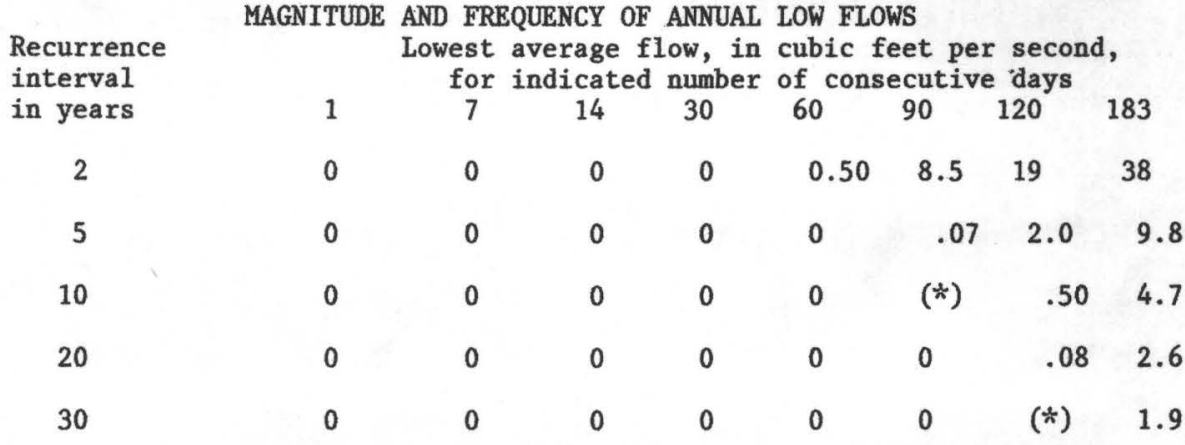

02232000 St. Johns River near Melbourne, Fla.

LOCATION.--Lat $28^{\circ} 05^{\prime} 04^{\prime \prime}$, long $80^{\circ} 45^{\prime} 08^{\prime \prime}$, in NW/ sec.5, T.28 S., R.36 E., Brevard County, Hydrologic Unit 03080101, near center of span on upstream side of bridge on U.S. Highway 192, 1.1 mi downstream from Sawgrass Lake, 1.7 mi upstream from Lake Washington, $9.2 \mathrm{mi}$ west of Melbourne and $262 \mathrm{mi}$ upstream from mouth.

DRAINAGE AREA. $--968 \mathrm{mi}^{2}$.

PERIOD OF RECORD ANALYZED.---July 1940 to June 1977.

REMARKS.--Flow occasionally reverses as a result of tide and wind effect. Negative discharges treated as zero values.

\begin{tabular}{|c|c|c|c|c|c|c|c|c|}
\hline $\begin{array}{l}\text { Recurrence } \\
\text { interval }\end{array}$ & MAGNIT & $\begin{array}{l}\text { AND FREQ } \\
\text { Lowest a } \\
\text { for } i\end{array}$ & $\begin{array}{l}\text { NCY } \\
\text { rag } \\
\text { ica }\end{array}$ & $\begin{array}{l}\text { ANNí } \\
\text { low, } \\
\text { numb }\end{array}$ & $\begin{array}{l}\text { LOW FI } \\
\text { cubic } \\
\text { of con }\end{array}$ & WS & $\begin{array}{l}r \text { secon } \\
\text { e days }\end{array}$ & \\
\hline in years & 1 & 7 & 14 & 30 & 60 & 90 & $120^{\circ}$ & 183 \\
\hline 2 & 30 & 35 & 39 & 50 & 64 & 92 & 143 & 221 \\
\hline 5 & 0 & 3.0 & 9 & 11 & 20 & 31 & 47 & 8 \\
\hline 10 & 0 & 0 & 0 & 0 & 8.0 & 15 & 22 & \\
\hline 20 & 0 & 0 & 0 & 0 & .40 & 8.2 & 11 & \\
\hline 30 & 0 & 0 & 0 & 0 & 0 & 5.2 & 6.7 & \\
\hline 50 & 0 & 0 & 0 & 0 & 0 & 3.7 & 4.7 & \\
\hline
\end{tabular}

* Less than $0.05 \mathrm{ft}^{3} / \mathrm{s}$. 
LOCATION.--Lat $28^{\circ} 12^{\prime} 46^{\prime \prime}$, long $80^{\circ} 54^{\prime} 40^{\prime \prime}$, in NW sec.22, T.26 S., R.34 E., Osceola County, Hydrologic Unit

03080101 , near right bank on upstream side of bridge on State Highway 419, 2.9 mi upstream from mouth and

8.5 mi north of Deer Park.

DRAINAGE AREA. $--25.7 \mathrm{mi}^{2}$.

PERIOD OF RECORD ANALYZED.--July 1956 to June 1977.

REMARKS.--Flow regulated to some extent since October 1970 by the construction of Jane Green Reservoir; levees were constructed and an interconnecting canal was dug joining the watershed areas of Taylor Creek, Pennywash Creek, Cox Creek and Wolf Creek.

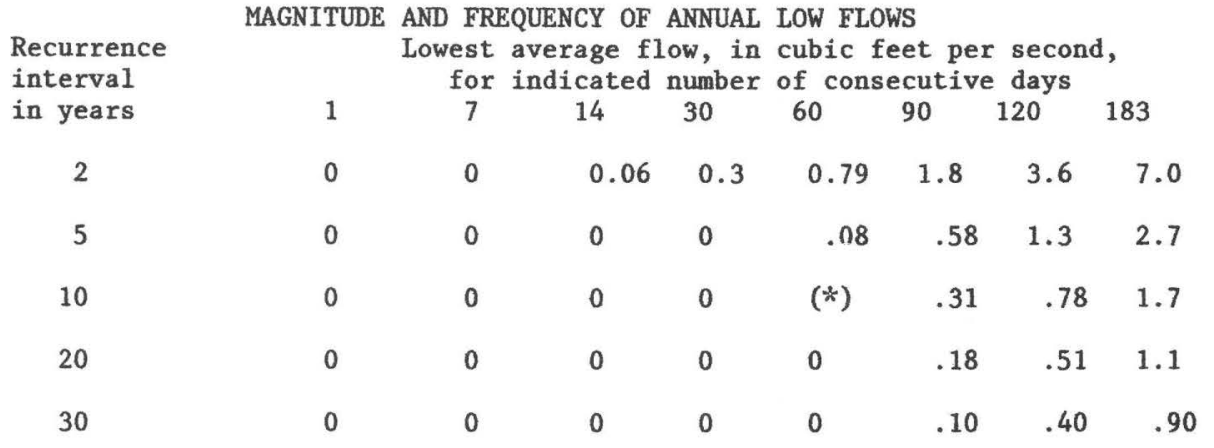

02232400 St. Johns River near Cocoa, Fla.

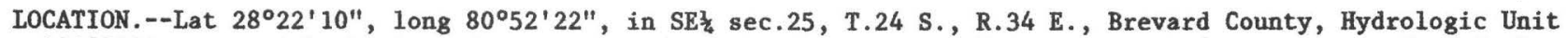

03080101 , near right bank on downstream side of bridge on State Highway 520, 0.6 mi upstream from Taylor Creek, 0.7 mi downstream from outlet of Lake Poinsett, $8.8 \mathrm{mi}$ west of Cocoa, and 232 mi upstream from mouth.

DRAINAGE AREA. $--1,331 \mathrm{mi}^{2}$.

PERIOD OF RECORD ANALYZED.--July 1954 to June 1977.

REMARKS.--Records include inflow from Taylor Creek.

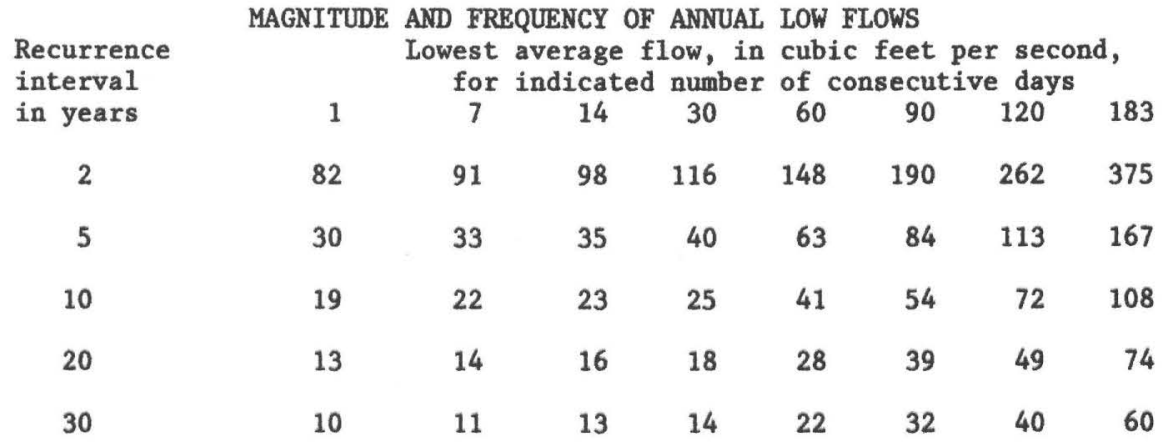

* Less than $0.05 \mathrm{ft}^{3} / \mathrm{s}$. 
ST. JOHNS RIVER

ST. JOHNS RIVER BASIN ABOVE OKLAWAHA RIVER

02232500 St. Johns River near Christmas, Fla.

LOCATION.--Lat $28^{\circ} 32^{\prime} 34^{\prime \prime}$, long $80^{\circ} 56^{\prime} 37^{\prime \prime}$, in SW/ sec.29, T.22 S., R.34 E., Orange County, Hydrologic Unit 03080101, on downstream side of bridge on State Highway $50,0.3 \mathrm{mi}$ upstream from Tootoosahatchee Creek, 2 mi upstream from Lake Cone, $4.5 \mathrm{mi}$ east of Christmas, and $209 \mathrm{mi}$ upstream from mouth. Prior to Sept. 1, 1976, at site 150 ft downstream.

DRAINAGE AREA.--1,539 $\mathrm{mi}^{2}$, includes that of Tootoosahatchee Creek.

PERIOD OF RECORD ANALYZED.--July 1934 to June 1977.

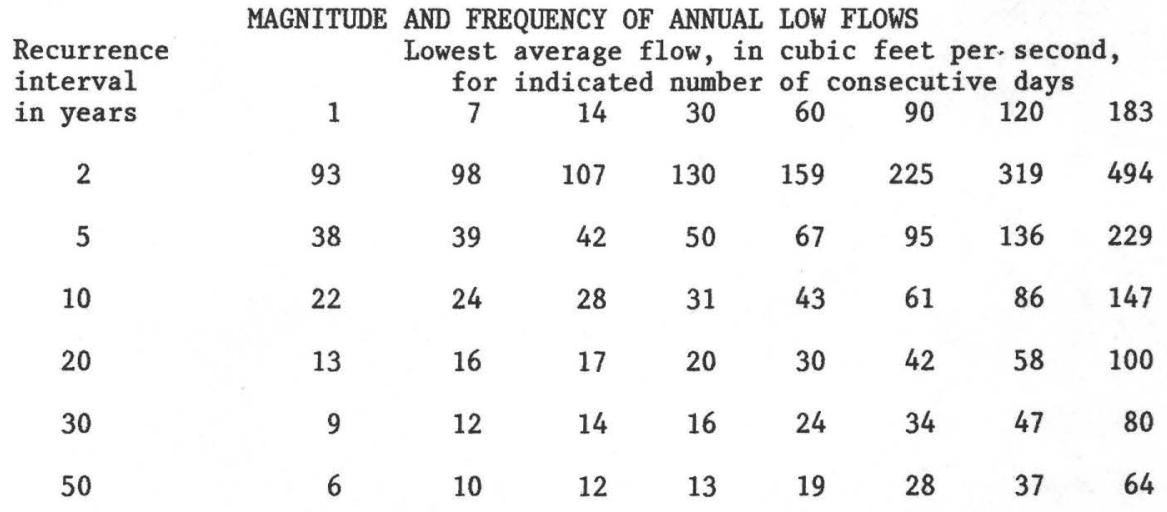

02233200 Little Econlockhatchee River near Union Park, Fla.

LOCATION.--Lat $28^{\circ} 31^{\prime} 29^{\prime \prime}$, long $81^{\circ} 1^{\prime} 39^{\prime \prime}$, in SW near right bank on downstream side of bridge on Berry-Deese Road, 3,300 ft upstream from a tributary, $3 \mathrm{mi}$ south of Union Park, $8.5 \mathrm{mi}$ east of Orlando, and $13 \mathrm{mi}$ upstream from mouth.

DRAINAGE AREA. - - $27.1 \mathrm{mi}^{2}$.

PERIOD OF RECORD ANALYZED.---July 1960 to June 1977.

\begin{tabular}{|c|c|c|c|c|c|c|c|c|}
\hline \multirow{2}{*}{$\begin{array}{l}\text { Recurrence } \\
\text { interval } \\
\text { in years }\end{array}$} & MAGNITUDE & $\begin{array}{l}\text { D FREQU } \\
\text { west av } \\
\text { for in }\end{array}$ & $\begin{array}{l}\text { ICY OF } \\
\text { rage } f \\
\text { icated }\end{array}$ & $\begin{array}{l}\text { ANNUAL } \\
\text { ow, in } \\
\text { number }\end{array}$ & $\begin{array}{l}\text { LOW FLC } \\
\text { cubic } t \\
\text { of cons }\end{array}$ & $\begin{array}{l}\text { WS } \\
\text { eet pe } \\
\text { ecutiv }\end{array}$ & $\begin{array}{l}\text { I secon } \\
\text { days }\end{array}$ & \\
\hline & 1 & 7 & 14 & 30 & 60 & 90 & 120 & 183 \\
\hline 2 & 1.5 & 1.8 & 1.9 & 2.4 & 3.2 & 4.5 & 6.8 & 9.0 \\
\hline 5 & .52 & .78 & .89 & 1.1 & 1.5 & 2.1 & 3.3 & 4.9 \\
\hline 10 & .27 & .49 & .57 & .71 & 1.0 & 1.4 & 2.3 & 3.6 \\
\hline 20 & .13 & .30 & .40 & .49 & .71 & 1.0 & 1.9 & 2.7 \\
\hline
\end{tabular}


ST. JOHNS RIVER

ST. JOHNS RIVER BASIN ABOVE OKLAWAHA RIVER

02233500 Econlockhatchee River near Chuluota, Fla.

LOCATION.--Lat $28^{\circ} 40^{\prime} 40^{\prime \prime}$, long $81^{\circ} 06^{\prime} 51^{\prime \prime}$, in SW $\frac{1}{4}$ sec.10, T.21 S., R.32 E., Seminole County, Hydrologic Unit 03080101 , near right bank on downstream side of bridge on State Highway 13, 2.6 mi northeast of Chuluota and 10 mi upstream from mouth.

DRAINAGE AREA. - - $241 \mathrm{mi}^{2}$.

PERIOD OF RECORD ANALYZED.--July 1936 to June 1977.

REMARKS.--Records include some flow diverted from Lake Mary Jane in the Kissimmee River Basin through Disston Canal.

\begin{tabular}{|c|c|c|c|c|c|c|c|c|}
\hline \multirow{2}{*}{$\begin{array}{l}\text { Recurrence } \\
\text { interval } \\
\text { in years }\end{array}$} & \multirow{2}{*}{$\begin{array}{c}\text { MAGNITUDE } \\
1\end{array}$} & $\begin{array}{l}\text { ND FREQ } \\
\text { owest a } \\
\text { for } i\end{array}$ & $\begin{array}{l}\text { NCY OF } \\
\text { rage f } \\
\text { licated }\end{array}$ & $\begin{array}{l}\text { ANNUAL } \\
\text { Low, in } \\
\text { number }\end{array}$ & $\begin{array}{l}\text { LOW } \\
\text { cub: } \\
\text { of }\end{array}$ & $\begin{array}{l}\text { OWS } \\
\text { feet } \\
\text { secut }\end{array}$ & $\begin{array}{l}\text { r sec } \\
\text { e day }\end{array}$ & \\
\hline & & 7 & 14 & 30 & 60 & 90 & 120 & 183 \\
\hline 2 & 19 & 21 & 22 & 25 & 32 & 43 & 56 & 85 \\
\hline 5 & 13 & 14 & 15 & 17 & 20 & 25 & 31 & 46 \\
\hline 10 & 10 & 11 & 12 & 13 & 16 & 18 & 22 & 34 \\
\hline 20 & 8.6 & 9.1 & 9.8 & 11 & 13 & 14 & 17 & 27 \\
\hline 30 & 7.8 & 8.2 & 8.6 & 9.5 & 11 & 12 & 15 & 23 \\
\hline 50 & 7.0 & 7.2 & 7.4 & 8.0 & 10 & 11 & 13 & 20 \\
\hline
\end{tabular}

02235000 Wekiva River near Sanford, Fla.

LOCATION.--Lat $28^{\circ} 48^{\prime} 54^{\prime \prime}$, long $81^{\circ} 25^{\prime} 10^{\prime \prime}$, in SE $\frac{1}{4}$ sec.21, T.19 S., R.29 E., Seminole County, Hydrologic Unit 03080101 , near right bank at downstream side of bridge on State Highway $46,4.5$ mi downstream from Little Wekiva River, $6.7 \mathrm{mi}$ upstream from mouth, and $8.9 \mathrm{mi}$ west of Sanford.

DRAINAGE AREA. $--189 \mathrm{mi}^{2}$.

PERIOD OF RECORD ANALYZED.--July 1936 to June 1977.

REMARKS.--Flow includes large ground-water inflow.

\begin{tabular}{|c|c|c|c|c|c|c|c|c|}
\hline $\begin{array}{l}\text { Recurrence } \\
\text { interval }\end{array}$ & MAGNIT & $\begin{array}{r}\text { ND } \\
\text { owes } \\
\text { fo }\end{array}$ & $\begin{array}{l}\text { UENCY } \\
\text { verag } \\
\text { ndica }\end{array}$ & $\begin{array}{l}\text { ANN } \\
\text { low, } \\
\text { numl }\end{array}$ & $\begin{array}{l}\text { LOW } \\
\text { cub } \\
\text { of }\end{array}$ & $\begin{array}{l}\text { OWS } \\
\text { feet } \\
\text { secu }\end{array}$ & $\begin{array}{l}r \mathrm{se} \\
\mathrm{e} \mathrm{da}\end{array}$ & \\
\hline in years & 1 & 7 & 14 & 30 & 60 & 90 & 120 & 183 \\
\hline 2 & 188 & 195 & 199 & 201 & 209 & 218 & 227 & 240 \\
\hline 5 & 164 & 171 & 174 & 178 & 187 & 194 & 201 & 212 \\
\hline 10 & 150 & 156 & 159 & 166 & 178 & 184 & 189 & 201 \\
\hline 20 & 138 & 143 & 146 & 156 & 171 & 176 & 180 & 194 \\
\hline 30 & 130 & 137 & 139 & 150 & 167 & 171 & 175 & 190 \\
\hline 50 & 124 & 126 & 131 & 145 & 163 & 167 & 171 & 187 \\
\hline
\end{tabular}


ST. JOHNS RIVER

ST. JOHNS RIVER BASIN ABOVE OKLAWAHA RIVER

02236000 St. Johns River near De Land, Fla.

LOCATION.--Lat $29^{\circ} 00^{\prime} 29^{\prime \prime}$, long $81^{\circ} 22^{\prime} 58^{\prime \prime}$, in land grant 38 , T.17 S., R.29 E., Lake County,

Hydrologic Unit 03080101 , near left bank on downstream side of Francis P. Whitehair Bridge on State Highway 44, 5 mi west of De Land and $142 \mathrm{mi}$ upstream from mouth.

DRAINAGE AREA. $--3,066 \mathrm{mi}^{2}$.

PERIOD OF RECORD ANALYZED.--July 1934 to June 1977.

REMARKS.--Flow occasionally reversed as a result of tide and wind effect. Negative discharges treated as zero values.

Recurrence
interval
in years
2
5
10
20
30
50

MAGNTTUDE AND FREQUENCY OF ANNUAL LOW FLOWS

Lowest average flow, in cubic feet per second, for indicated number of consecutive days

interval

$7 \quad 14$

60

$90 \quad 120$

183

$\begin{array}{llllllll}90 & 450 & 660 & 880 & 1060 & 1260 & 1490 & 1840\end{array}$

$\begin{array}{llllllll}0 & 0 & 45 & 430 & 581 & 777 & 936 & 1210\end{array}$

$\begin{array}{llllllll}0 & 0 & 0 & 140 & 380 & 550 & 724 & 960\end{array}$

$\begin{array}{llllllll}0 & 0 & 0 & 10 & 280 & 400 & 540 & 788\end{array}$

$\begin{array}{llllllll}0 & 0 & 0 & 0 & 150 & 320 & 430 & 670\end{array}$

0

0

0

$\begin{array}{lllll}0 & 110 & 280 & 380 & 600\end{array}$ 


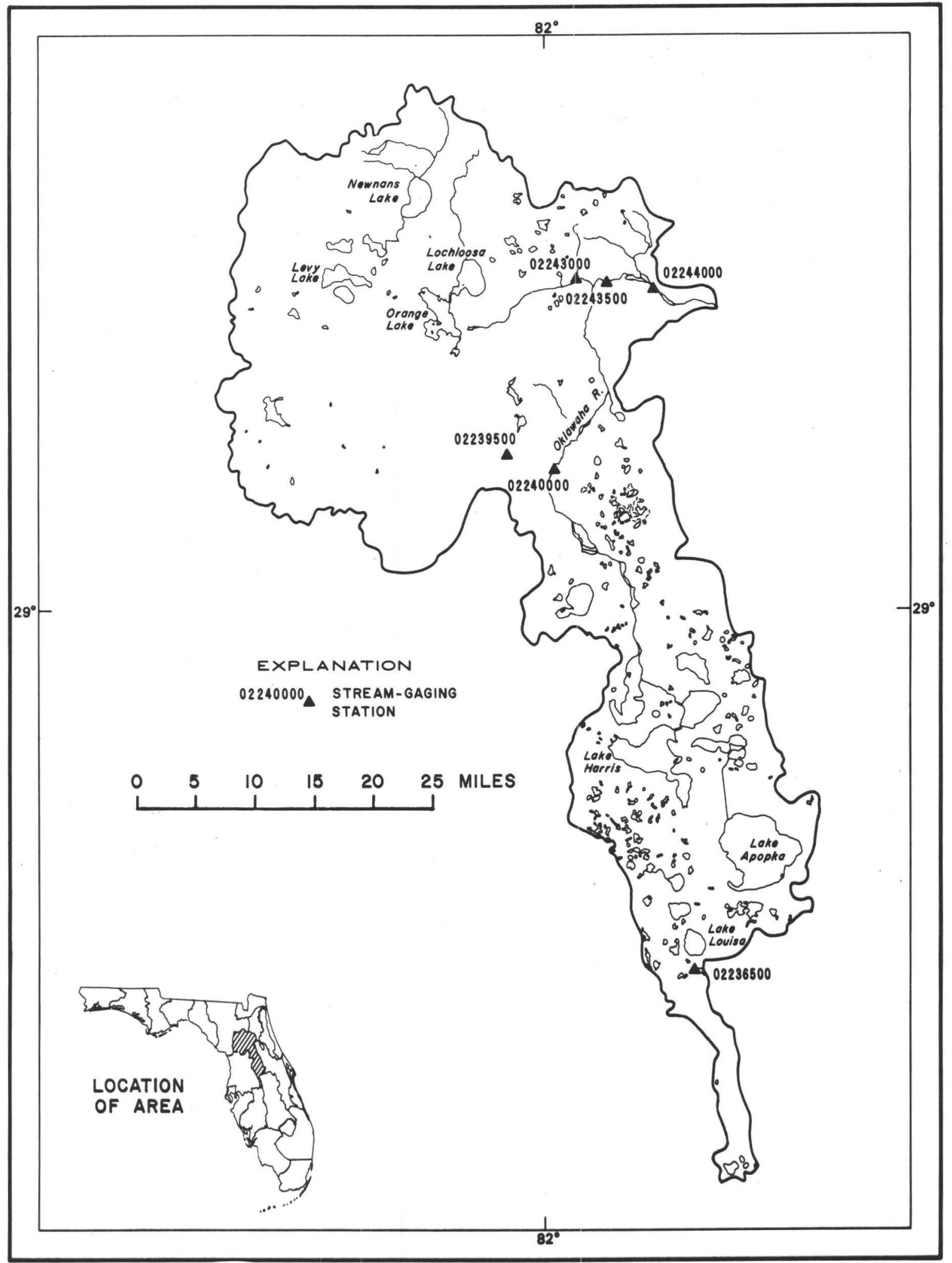

Figure 3.--Location of stream-gaging stations in the Oklawaha River basin. 
ST. JOHNS RIVER

OKLAWAHA RIVER BASIN

02236500

Big Creek near Clermont, Fla.

LOCATION.--Lat $28^{\circ} 26^{\prime} 51^{\prime \prime}$, long $81^{\circ} 4^{\prime} 4^{\prime \prime}$, in NE $\frac{1}{4}$ sec.31, T.23 S., R.26 E. Lake County, Hydrologic Unit 03080102, near left bank $40 \mathrm{ft}$ downstream from $\log$ bridge, $1 \mathrm{mi}$ upstream from Lake Louisa, 7.5 mi southeast of $\mathrm{Clermont,}$ and $131 \mathrm{mi}$ upstream from mouth.

DRAINAGE AREA. $--68 \mathrm{mi}^{2}$, approximately.

PERIOD OF RECORD ANALYZED.--July 1959 to June 1977.

\begin{tabular}{|c|c|c|c|c|c|c|c|c|}
\hline $\begin{array}{l}\text { Recurrence } \\
\text { interval }\end{array}$ & MAGNITUDE AN & $\begin{array}{l}D \text { FREQL } \\
\text { west av } \\
\text { for in }\end{array}$ & $\begin{array}{l}\text { JCY of } \\
\text { cage } f 1 \\
\text { icated }\end{array}$ & $\begin{array}{l}\text { ANNUAL } \\
\text { ow, in } \\
\text { number }\end{array}$ & $\begin{array}{l}\text { LOW FLO } \\
\text { cubic } f \\
\text { of cons }\end{array}$ & $\begin{array}{l}\text { WS } \\
\text { eet per } \\
\text { ecutive }\end{array}$ & $\begin{array}{l}\text { second } \\
\text { e days }\end{array}$ & \\
\hline in years & 1 & 7 & 14 & 30 & 60 & 90 & 120 & 183 \\
\hline 2 & 0 & 0.08 & 0.10 & 0.19 & 0.43 & 1.3 & 3.4 & 6.2 \\
\hline 5 & 0 & 0 & 0 & $(*)$ & .09 & .31 & .83 & 1.7 \\
\hline 10 & 0 & 0 & 0 & 0 & $(*)$ & .16 & .40 & .83 \\
\hline 20 & 0 & 0 & 0 & 0 & 0 & .09 & .22 & .46 \\
\hline
\end{tabular}

LOCATION.--Lat $29^{\circ} 11^{\prime}$, long $82^{\circ} 00^{\prime}$, in sec.15, T.15 S., R.23 E., Hydrologic Unit 03080102, on left bank about $15 \mathrm{ft}$ upstream from highway bridge known as Sharpes Ferry, 2 mi upstream from Silver River, and 9 mi east of Ocala, Marion County.

DRAINAGE AREA.--1,070 $\mathrm{mi}^{2}$, approximately .

PERIOD OF RECORD ANALYZED.--July 1930 to June 1968.

REMARKS.--Low flow regulated at Moss Bluff Dam 12 miles above station.

\begin{tabular}{|c|c|c|c|c|c|c|c|c|}
\hline $\begin{array}{l}\text { Recurrence } \\
\text { interval }\end{array}$ & MAGNITUDE & $\begin{array}{l}\text { ND FREC } \\
\text { owest } \\
\text { for }\end{array}$ & $\begin{array}{l}\text { JENCY OF } \\
\text { gerage } f \\
\text { dicated }\end{array}$ & $\begin{array}{l}\text { ANNUAL } \\
\text { low, in } \\
\text { number }\end{array}$ & $\begin{array}{l}\text { LOW F } \\
\text { cubic } \\
\text { of co }\end{array}$ & $\begin{array}{l}\text { OWS } \\
\text { feet p } \\
\text { secut i }\end{array}$ & $\begin{array}{l}\text { seco } \\
\text { days }\end{array}$ & \\
\hline in years & 1 & 7 & 14 & 30 & 60 & 90 & 120 & 183 \\
\hline 2 & 86 & 102 & 109 & 125 & 152 & 190 & 226 & 272 \\
\hline 5 & 30 & 36 & 40 & 47 & 61 & 79 & 97 & 115 \\
\hline 10 & 15 & 18 & 20 & 23 & 30 & 36 & 48 & 53 \\
\hline 20 & 7.6 & 9.0 & 10 & 13 & 16 & 19 & 22 & 25 \\
\hline 30 & 5.6 & 6.0 & 7.0 & 9.0 & 11 & 13 & 14 & 16 \\
\hline 50 & 3.6 & 4.5 & 5.0 & 6.4 & 7.6 & 8.8 & 9.2 & 10 \\
\hline
\end{tabular}

* Less than $0.05 \mathrm{ft}^{3} / \mathrm{s}$. 
ST. JOHNS RIVER

OKLAWAHA RIVER BASIN

02239500 Silver Springs near Ocala, Fla.

LOCATION.--Lat $29^{\circ} 12^{\prime} 44^{\prime \prime}$, long $82^{\circ} 03^{\prime} 15^{\prime \prime}$, in SE $\frac{1}{4}$ sec.1, T.15 S., R.23 E., Marion County, Hydrologic Unit 03080102, in canal at glass-bottom boat docking shed, 1,400 ft downstream from head of springs and $5.3 \mathrm{mi}$ northeast of Ocala.

PER-IOD OF RECORD ANALYZED.--July 1933 to June 1977.

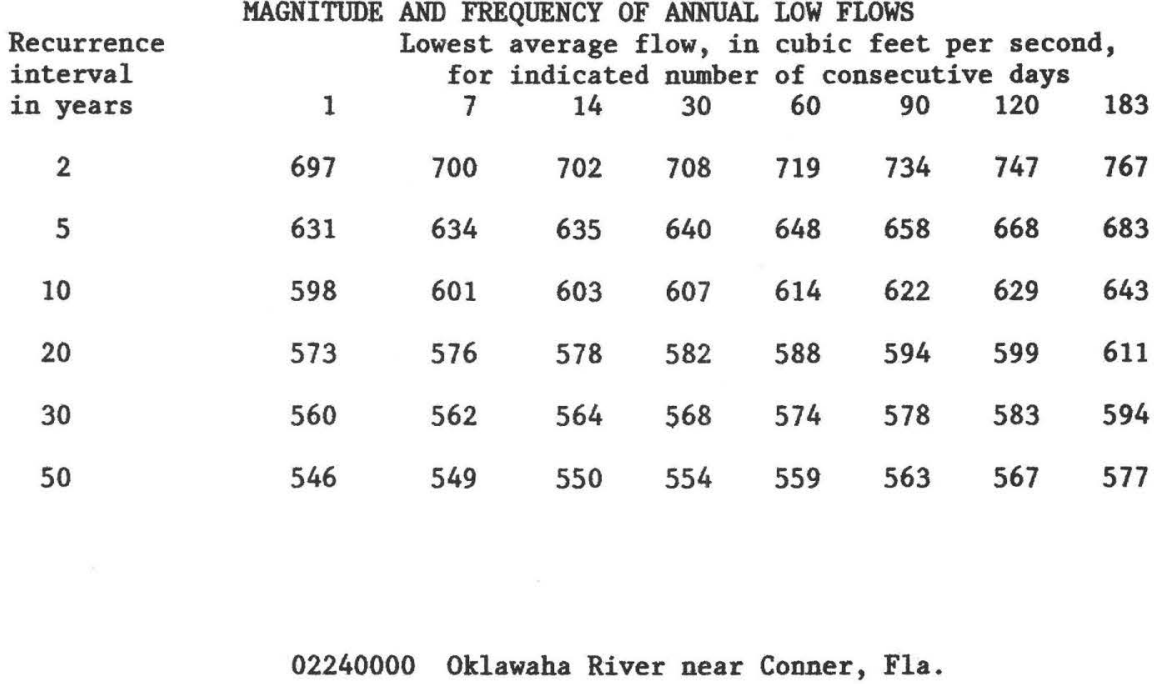

LOCATION.--Lat $29^{\circ} 13^{\prime}$, long $81^{\circ} 59^{\prime}$, in sec.3, T.15 S., R.23 E., Hydrologic Unit 03080102 , on right bank 15 ft downstream from bridge on State Highway 40, a quarter of a mile downstream from Silver River, about $1 \frac{1}{2}$ mi southwest of Conner, and $8 \mathrm{mi}$ east of Ocala.

DRAINAGE AREA. --1,180 $\mathrm{mi}^{2}$, approximately.

PERIOD OF RECORD ANALYZED.--July 1930 to June 1946.

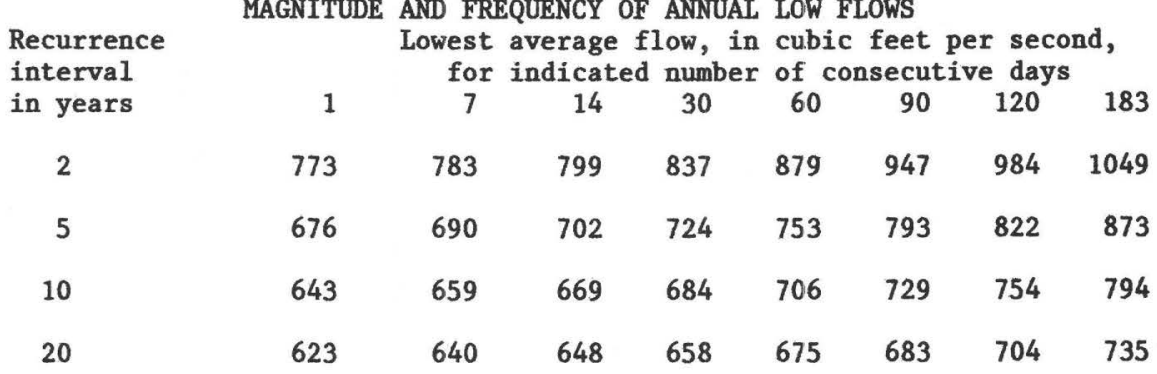


ST. JOHNS RIVER

OKLAWAHA RIVER BASIN

02240500 Oklawaha River at Eureka, Fla.

LOCATION.--Lat $29^{\circ} 22^{\prime}$ long $81^{\circ} 54^{\prime}$, in sec.9, T.13 S., R.24 E., Hydrologic Unit 03080102, on right bank $20 \mathrm{ft}$ upstream from bridge on State Highway 316 in Eureka, Marion County, and 3 mi downstream from Eaton Creek.

DRAINAGE AREA. $--1,420 \mathrm{mi}^{2}$, approximately.

PERIOD OF RECORD ANALYZED.--July 1930 to June 1934, July 1944 to June 1952.

\begin{tabular}{|c|c|c|c|c|c|c|c|c|}
\hline $\begin{array}{l}\text { Recurrence } \\
\text { interval }\end{array}$ & MAGNITUDE & $\begin{array}{l}\text { AND FR } \\
\text { Lowest } \\
\text { for }\end{array}$ & $\begin{array}{l}\text { EQUENCY } \\
\text { average } \\
\text { indicat }\end{array}$ & $\begin{array}{l}\text { F ANNU } \\
\text { flow, } \\
\text { d numb }\end{array}$ & $\begin{array}{l}\text { L LOW } \\
\text { in cub } \\
\text { r of }\end{array}$ & $\begin{array}{l}\text { FLOWS } \\
\text { c feet } \\
\text { onsecut }\end{array}$ & $\begin{array}{l}\text { per sec } \\
\text { ive day }\end{array}$ & nd, \\
\hline in years & 1 & 7 & 14 & 30 & 60 & 90 & 120 & 183 \\
\hline 2 & 946 & 968 & 983 & 1040 & 1080 & 1150 & $1200^{\circ}$ & 1300 \\
\hline 5 & 781 & 802 & 815 & 856 & 892 & 934 & 966 & 1040 \\
\hline 10 & 702 & 724 & 736 & 767 & 796 & 823 & 846 & 896 \\
\hline 20 & 642 & 664 & 675 & 697 & 720 & 734 & 751 & 783 \\
\hline
\end{tabular}

02243000 Orange Creek at Orange Springs, Fla.

LOCATION.--Lat $29^{\circ} 30^{\prime} 34^{\prime \prime}$, long $81^{\circ} 56^{\prime} 47^{\prime \prime}$, in NE sec.25, T.11 S., R.23 E., Marion County, Hydrologic Unit 03080102, near right bank at downstream side of bridge on State Highway 21, 0.2 mi northwest of Orange Springs and 1.2 mi upstream from Little Orange Creek.

DRAINAGE AREA.--1,067 $\mathrm{mi}^{2}$, includes Paynes Prairie, a diked sinkhole area of $650 \mathrm{mi}^{2}$, approximately, which is noncontributing except by pumpage.

PERIOD OF RECORD ANALYZED.--July 1943 to June 1952, July 1956 to June 1971, July 1975 to June 1977.

Recurrence
interval

in years

2

5

10

20

30
MAGNITUDE AND FREQUENCY OF ANNUAL LOW FLOWS

Lowest average flow, in cubic feet per second, for indicated number of consecutive days

$\begin{array}{llllllll}1 & 7 & 14 & 30 & 60 & 90 & 120 & 183\end{array}$

$\begin{array}{llllllll}12 & 13 & 15 & 18 & 25 & 36 & 49 & 72\end{array}$

$\begin{array}{llllllll}4.1 & 4.8 & 5.2 & 5.7 & 9.0 & 11 & 15 & 29\end{array}$

$\begin{array}{llllllll}3.1 & 3.5 & 3.8 & 4.3 & 6.0 & 6.8 & 11 & 18\end{array}$

$\begin{array}{llllllll}2.3 & 2.5 & 2.7 & 3.4 & 4.1 & 4.7 & 8.2 & 12\end{array}$

$\begin{array}{llllllll}1.9 & 2.0 & 2.1 & 2.9 & 3.1 & 3.5 & 6.6 & 9.0\end{array}$


ST. JOHIS RIVER

OKLAWAHA RIVER BASIN

02243500 Oklawaha River near Orange Springs, Fla.

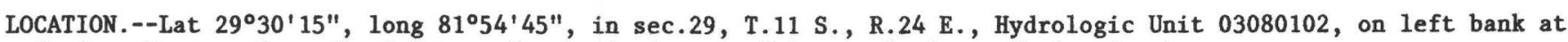
Jordans Ferry and mouth of Orange Creek, 2 mi east of Orange Springs, Marion County.

DRAINAGE AREA.--2,010 $\mathrm{mi}^{2}$, approximately (includes that of Orange Creek).

PERIOD OF RECORD ANALYZED.--July 1930 to June 1952.

\begin{tabular}{|c|c|c|c|c|c|c|c|c|}
\hline \multirow{2}{*}{$\begin{array}{l}\text { Recurrence } \\
\text { interval } \\
\text { in years }\end{array}$} & MAGNITUD & $\begin{array}{l}\text { AND FR } \\
\text { Lowest } \\
\text { for }\end{array}$ & $\begin{array}{l}\text { QUENCY } \\
\text { average } \\
\text { indicat }\end{array}$ & $\begin{array}{l}\text { F ANNT } \\
\text { flow, } \\
\text { d numb }\end{array}$ & $\begin{array}{l}\text { L LOW } \\
\text { n cub } \\
\mathbf{r} \text { of }\end{array}$ & $\begin{array}{l}\text { LOWS } \\
\text { feet } \\
\text { nsecut }\end{array}$ & $\begin{array}{l}\text { er se } \\
\text { ve day }\end{array}$ & nd, \\
\hline & 1 & 7 & 14 & 30 & 60 & 90 & 120 & 183 \\
\hline 2 & 956 & 981 & 998 & 1060 & 1120 & 1210 & 1280 & 1400 \\
\hline 5 & 801 & 819 & 831 & 870 & 909 & 962 & 1010 & 1100 \\
\hline 10 & 738 & 752 & 763 & 789 & 821 & 859 & 899 & 973 \\
\hline 20 & 694 & 705 & 714 & 732 & 756 & 784 & 820 & 881 \\
\hline 30 & 670 & 680 & 690 & 700 & 720 & 745 & 780 & 830 \\
\hline
\end{tabular}

02244000 Oklawaha River at Riverside Landing, near Orange Springs, Fla.

LOCATION.--Lat $29^{\circ} 30^{\prime}$, long $81^{\circ} 48^{\prime}$, in sec.33, T.11 S., R.25 E., Hydrologic Unit 03080102, on right bank near boat dock at Riverside Landing, $8 \frac{1}{4}$ mi east of Orange Springs, Marion County.

DRAINAGE AREA. $--2,100 \mathrm{mi}^{2}$, approximately.

PERIOD OF RECORD ANALYZED.--July 1944 to June 1968.

\begin{tabular}{|c|c|c|c|c|c|c|c|c|}
\hline $\begin{array}{l}\text { Recurrence } \\
\text { interval }\end{array}$ & MAGNITU & $\begin{array}{l}\text { AND FR } \\
\text { Lowest } \\
\text { for }\end{array}$ & $\begin{array}{l}\text { QUENCY } \\
\text { average } \\
\text { indicat }\end{array}$ & $\begin{array}{l}\text { F ANN } \\
\text { flow, } \\
\text { d numl }\end{array}$ & $\begin{array}{l}\text { AL LOW } \\
\text { in cub } \\
\text { er of }\end{array}$ & $\begin{array}{l}\text { LOWS } \\
\text { feet } \\
\text { nsecut }\end{array}$ & $\begin{array}{l}\text { er sec } \\
\text { ve day }\end{array}$ & \\
\hline in years & 1 & 7 & 14 & 30 & 60 & 90 & 120 & 183 \\
\hline 2 & 1100 & 1120 & 1150 & 1190 & 1270 & 1370 & 1460 & 1620 \\
\hline 5 & 863 & 888 & 900 & 929 & 973 & 1030 & 1070 & 1160 \\
\hline 10 & 767 & 788 & 796 & 818 & 849 & 884 & 914 & 976 \\
\hline 20 & 696 & 716 & 721 & 738 & 760 & 781 & 800 & 842 \\
\hline 30 & 660 & 679 & 684 & 698 & 71.6 & 730 & 740 & 780 \\
\hline
\end{tabular}




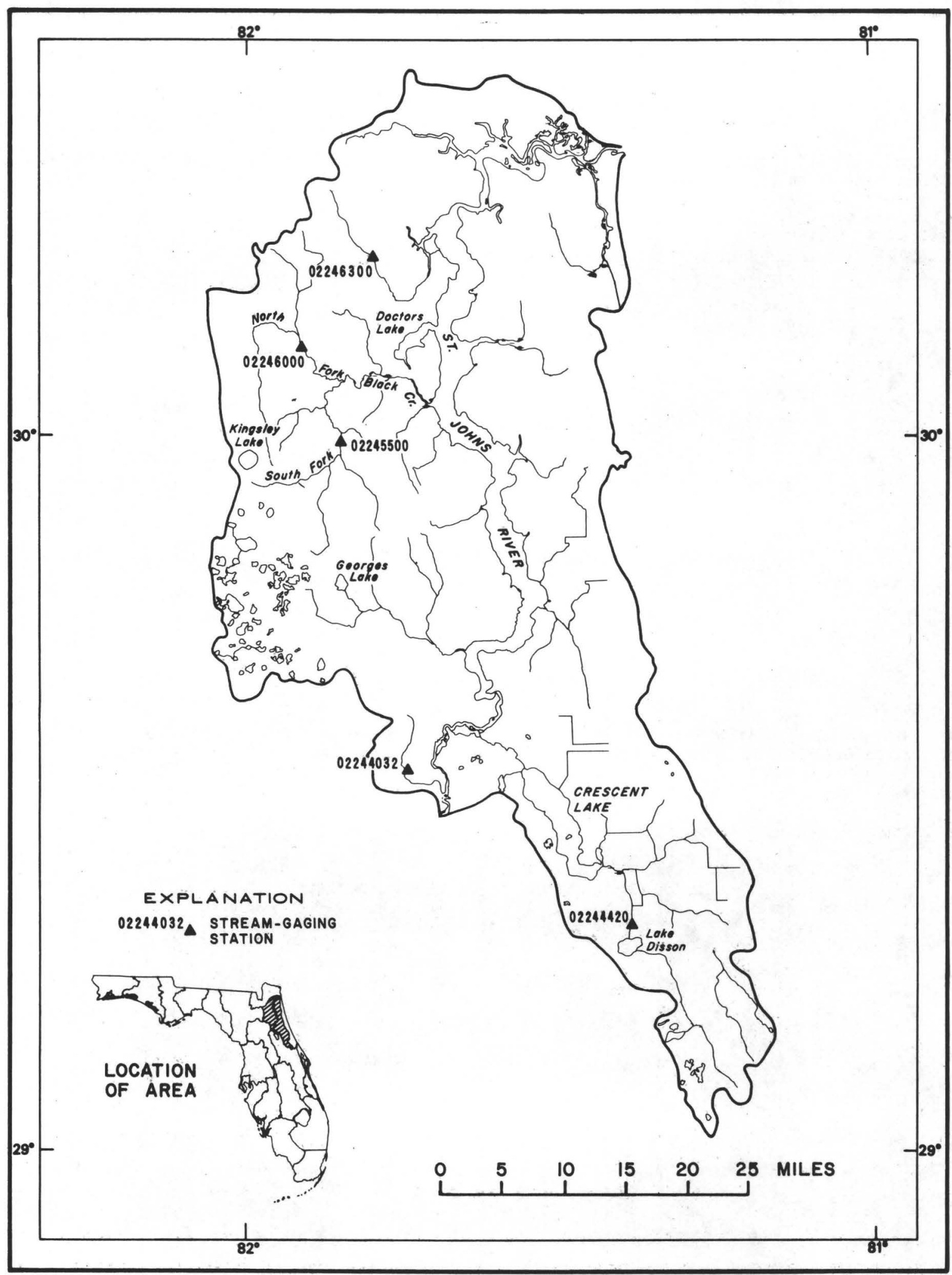

Figure 4.--Location of stream-gaging stations in the St. Johns River basin below the Oklawaha River. 
LOCATION.--Lat $29^{\circ} 19^{\prime} 20^{\prime \prime}$, long $81^{\circ} 23^{\prime} 10^{\prime \prime}$, in SE⿺ sec. 32, T.13 S., R. 29 E., Flagler County, Hydrologic Unit 03080103, on right bank $600 \mathrm{ft}$ downstream from bridge on State Highway 305, 1.4 mi downstream from Lake Disston, and $6.4 \mathrm{mi}$ east of Seville.

DRAINAGE AREA. $--93.0 \mathrm{mi}^{2}$.

PERIOD OF RECORD ANALYZED.--July 1952 to June 1978.

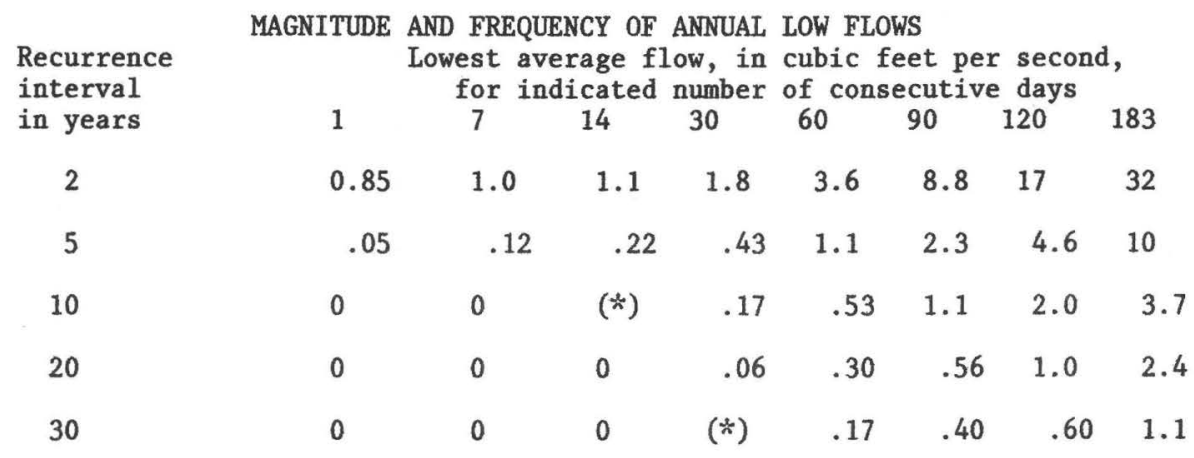

02245500 South Fork Black Creek near Penney Farms, Fla.

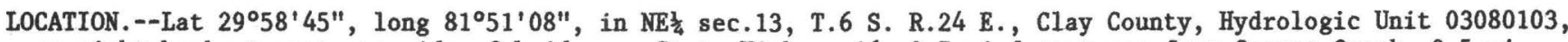
on right bank at upstream side of bridge on State Highway 16, 0.7 mi downstream from Greens Creek, $2.5 \mathrm{mi}$ west of Penney Farms, 9.5 mi west of Green Cove Springs, and 24 mi upstream from mouth of Black Creek.

DRAINAGE AREA. $--134 \mathrm{mi}^{2}$.

PERIOD OF RECORD ANALYZED.--April 1940 to March 1978.

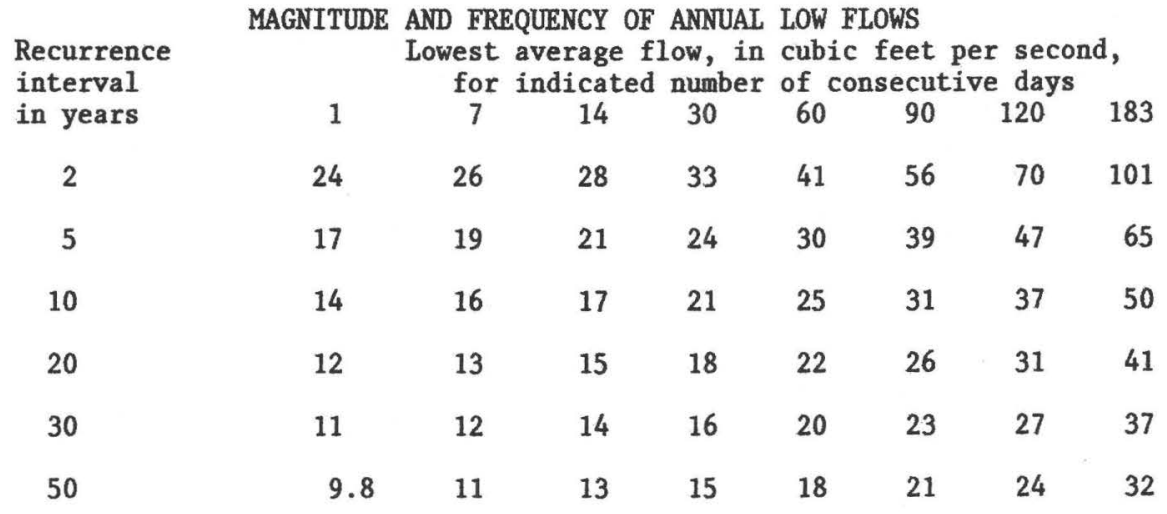

* Less than $0.05 \mathrm{ft}^{3} / \mathrm{s}$. 
ST. JOHNS RIVER

ST. JOHNS RIVER BASIN BELOW OKLAWAHA RIVER

02246000 North Fork Black Creek near Middleburg, Fla.

LOCATION.--Lat $30^{\circ} 06^{\prime} 47^{\prime \prime}$, long $81^{\circ} 54^{\prime} 24^{\prime \prime}$, in NE $\frac{1}{4}$ sec.33, T.4 S., R.24 E., Clay County, Hydrologic Unit 03080103, on left bank $0.3 \mathrm{mi}$ upstream from Big Branch, $4 \mathrm{mi}$ northwest of Middleburg, and 7.5 mi upstream from confluence with South Fork.

DRAINAGE AREA. --177 $\mathrm{mi}^{2}$.

PERIOD OF RECORD ANALYZED.--April 1932 to March 1977.

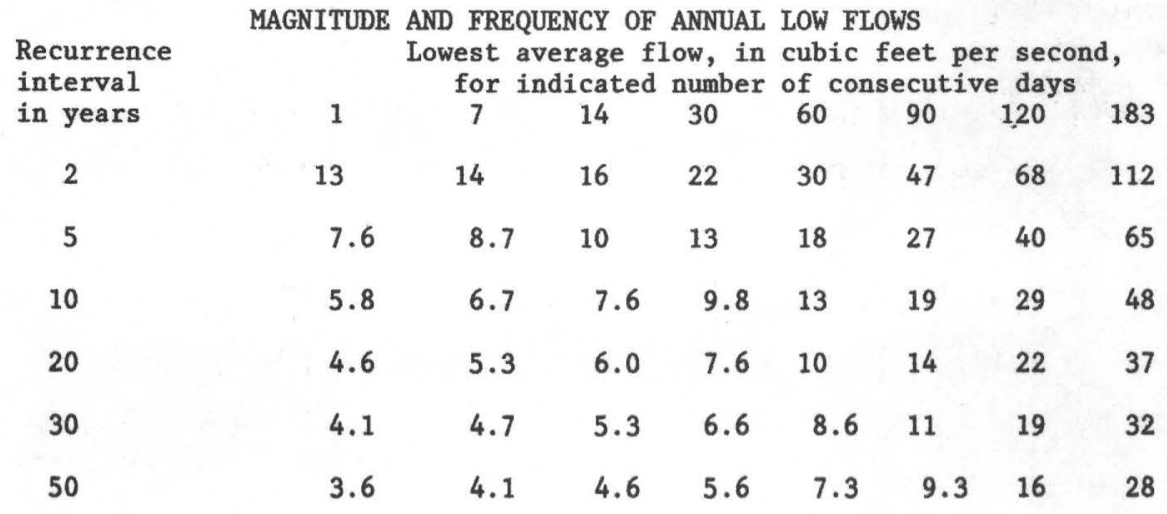

02246300 Ortega River at Jacksonville, Fla.

LOCATION.--Lat $30^{\circ} 14^{\prime} 50^{\prime \prime}$, long $81^{\circ} 47^{\prime} 49^{\prime \prime}$, in NWh sec.15, T.3 S., R.25 E., Duval County, Hydrologic Unit 03080103, near center of span on downstream side of bridge on 103rd Street in Jacksonville, 15 mi upstream from mouth.

DRAINAGE AREA. $--30.9 \mathrm{mi}^{2}$.

PERIOD OF RECORD ANALYZED.--April 1965 to March 1978.

\begin{tabular}{|c|c|c|c|c|c|c|c|c|}
\hline $\begin{array}{l}\text { Recurrence } \\
\text { interval }\end{array}$ & MAGNITUDE & $\begin{array}{c}\text { AND FREQL } \\
\text { Lowest av } \\
\text { for in }\end{array}$ & $\begin{array}{l}\text { CY of } \\
\text { age fl } \\
\text { cated }\end{array}$ & $\begin{array}{l}\text { ANNUAL } \\
\text { ow, in } \\
\text { number }\end{array}$ & $\begin{array}{l}\text { LOW FI } \\
\text { cubic } \\
\text { of con }\end{array}$ & $\begin{array}{l}\text { WS } \\
\text { feet pe } \\
\text { ecutiv }\end{array}$ & $\begin{array}{l}r \text { secon } \\
\text { e days }\end{array}$ & \\
\hline in years & 1 & & & 30 & & 90 & 120 & 183 \\
\hline 2 & 1.1 & 1.3 & 1.6 & 2.4 & 3.6 & 6.5 & 10 & 17 \\
\hline 5 & .56 & .68 & .86 & 1.2 & 2.2 & 3.7 & 5.7 & 11 \\
\hline 10 & .40 & .47 & .58 & .90 & 1.6 & 2.5 & 3.8 & 9.1 \\
\hline 20 & .30 & .34 & .41 & .67 & 1.3 & 1.8 & 2.5 & 7.7 \\
\hline
\end{tabular}




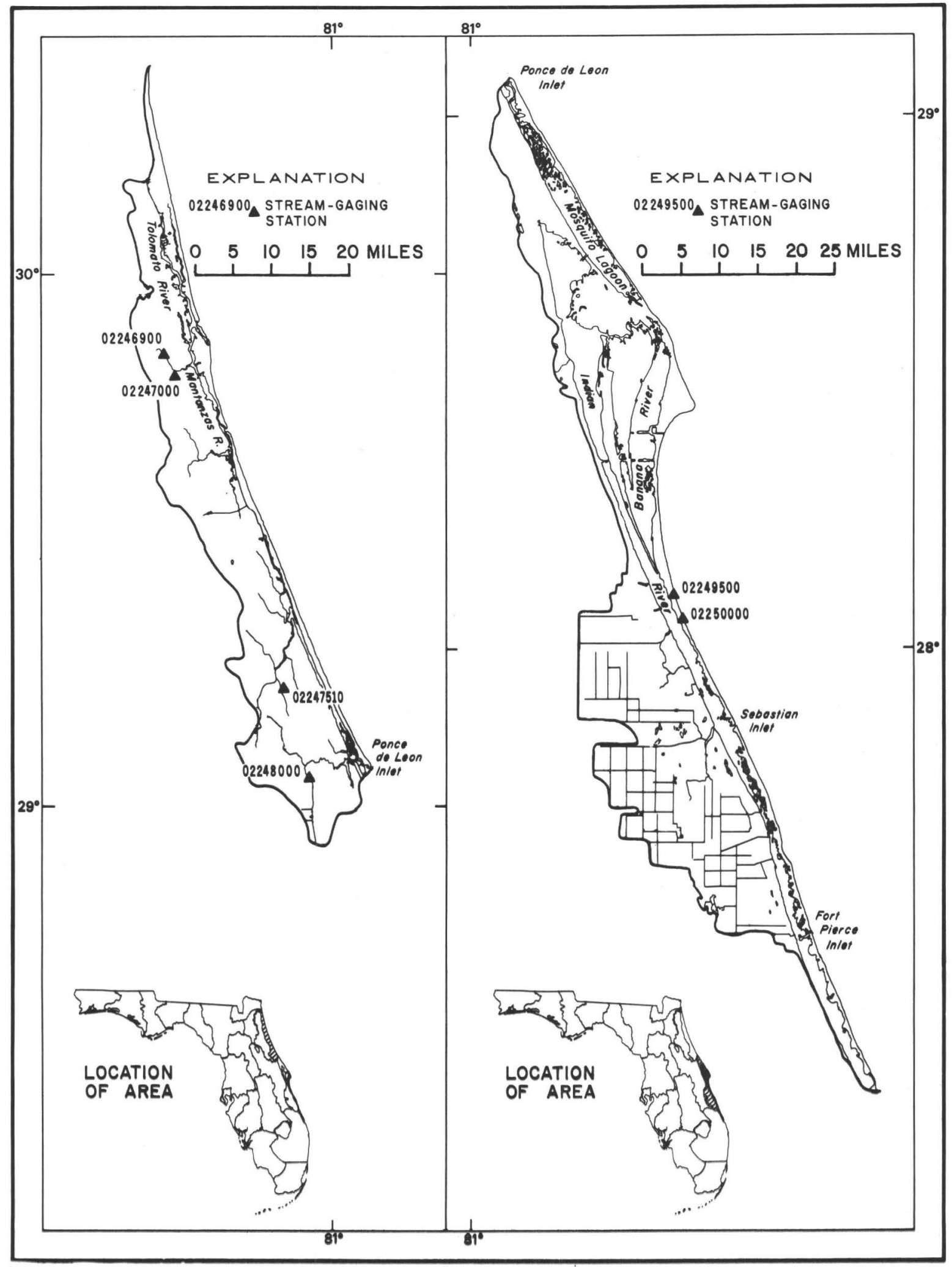

Figure 5.--Location of stream-gaging stations in the coastal area between the St. Johns and St. Lucie Rivers. 
COASTAL AREA BETWEEN ST. JOHNS AND ST. LUCIE RIVERS

02246900 Moultrie Creek at State Highway 207, near St. Augustine, Fla.

LOCATION.--Lat $29^{\circ} 50^{\prime} 50^{\prime \prime}$, long $81^{\circ} 21^{\prime} 39^{\prime \prime}$, in SE $\frac{1}{4}$ sec.34, T.7 S., R.29 E., St. Johns County, Hyrologic Unit 03080201 , at center on downstream side of box culverts on State Highway 207, 2.0 mi upstream from Fort Peyton Branch, $4.2 \mathrm{mi}$ southwest of $\mathrm{St}$. Augustine, and $6.3 \mathrm{mi}$ upstream from mouth.

DRAINAGE AREA. $--19.8 \mathrm{mi}^{2}$.

PERIOD OF RECORD ANALYZED.--July 1962 to June 1978.

Recurrence
interval
in years
2
5
10
20

MAGNITUDE AND FREQUENCY OF ANNUAL LOW FLOWS

Lowest average flow, in cubic feet per second, for indicated number of consecutive days

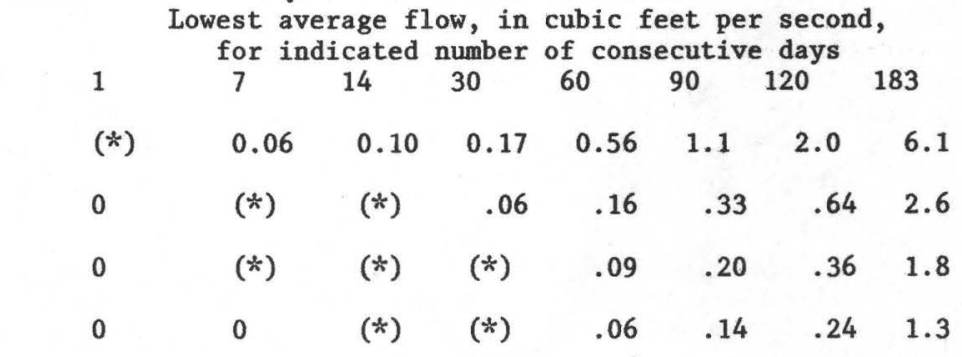

02247000 Moultrie Creek near St. Augustine, Fla.

LOCATION.--Lat $29^{\circ} 49^{\prime} 40^{\prime \prime}$, long $81^{\circ} 20^{\prime} 57^{\prime \prime}$, in sec.11, T.8 S., R.29 E., Hydrologic Unit 03080201, on right bank $0.4 \mathrm{mi}$ upstream from Fort Peyton Branch, $1.6 \mathrm{mi}$ downstream from bridge on State Highway 207 , and $4.9 \mathrm{mi}$ southwest of St. Augustine, St. Johns County.

DRAINAGE AREA. $--23.3 \mathrm{mi}^{2}$.

PERIOD OF RECORD ANALYZED.--July 1940 to June 1964.

\begin{tabular}{|c|c|c|c|c|c|c|c|c|}
\hline $\begin{array}{l}\text { Recurrence } \\
\text { interval }\end{array}$ & MAGNITUDE & $\begin{array}{l}\text { D FREQL } \\
\text { west a } \\
\text { for in }\end{array}$ & $\begin{array}{l}\text { CY OF } \\
\text { age } f 1 \\
\text { cated }\end{array}$ & $\begin{array}{l}\text { ANNUAL } \\
\text { ow, in } \\
\text { number }\end{array}$ & $\begin{array}{l}\text { LOW FLC } \\
\text { cubic } \\
\text { of cons }\end{array}$ & $\begin{array}{l}\text { WS } \\
\text { eet per } \\
\text { ecutive }\end{array}$ & $\begin{array}{l}\text { seconc } \\
\text { days }\end{array}$ & \\
\hline in years & 1 & 7 & 14 & 30 & 60 & 90 & 120 & 183 \\
\hline 2 & 0.42 & 0.45 & 0.60 & 0.75 & 0.89 & 1.8 & 3.2 & 7.4 \\
\hline 5 & .31 & .40 & .46 & .52 & .56 & .80 & 1.3 & 2.8 \\
\hline 10 & .27 & .30 & .36 & .43 & .47 & .54 & .84 & 1.7 \\
\hline 20 & .22 & .28 & .30 & .32 & .39 & .42 & .61 & 1.1 \\
\hline 30 & .18 & .25 & .28 & .29 & .33 & .36 & .52 & .80 \\
\hline
\end{tabular}

\footnotetext{
* Less than $0.05 \mathrm{ft}^{3} / \mathrm{s}$.
} 
LOCATION.--Lat $29^{\circ} 13^{\prime} 02^{\prime \prime}$, long $81^{\circ} 06^{\prime} 32^{\prime \prime}$, in NW/ sec.9, T.15 S., R.32 E., Volusia County, Hydrologic Unit 03080201 , near center of span on downstream side of bridge on 11th Street extension, 0.3 mi southwest of Interstate 95, 2 mi upstream from Priest Branch, $4.5 \mathrm{mi}$ southwest of Holly Hill, and 12 mi upstream from mouth.

DRAINAGE AREA. $--76.8 \mathrm{mi}^{2}$.

PERIOD OF RECORD ANALYZED.--July 1966 to June 1977.

\begin{tabular}{|c|c|c|c|c|c|c|c|c|}
\hline $\begin{array}{l}\text { Recurrence } \\
\text { interval }\end{array}$ & MAGNITUDE & $\begin{array}{l}\text { D FREQ } \\
\text { west a } \\
\text { for i. }\end{array}$ & $\begin{array}{l}\text { NCY OF } \\
\text { rage } f \\
\text { icated }\end{array}$ & $\begin{array}{l}\text { ANNUAL } \\
\text { low, in } \\
\text { number }\end{array}$ & $\begin{array}{l}\text { LOW FL } \\
\text { cubic } \\
\text { of con }\end{array}$ & $\begin{array}{l}\text { WS } \\
\text { eet p } \\
\text { ecuti }\end{array}$ & $\begin{array}{l}r \text { seco } \\
\text { e days }\end{array}$ & \\
\hline in years & 1 & 7 & 14 & 30 & 60 & 90 & 120 & 183 \\
\hline 2 & 0.80 & 1.0 & 1.1 & 1.4 & 2.8 & 4.8 & 8.7 & 16 \\
\hline 5 & .23 & .40 & .45 & .62 & 1.4 & 2.4 & 3.7 & 6.0 \\
\hline 10 & .11 & .20 & .28 & .42 & 1.0 & 1.7 & 2.3 & 3.5 \\
\hline 20 & 0 & .10 & .18 & .30 & .72 & 1.3 & 1.5 & 2.2 \\
\hline
\end{tabular}

LOCATION.--Lat $29^{\circ} 03^{\prime} 01^{\prime \prime}$, long $81^{\circ} 02^{\prime} 49^{\prime \prime}$, in SE $\frac{3}{4}$ sec.1, T.17 S., R.32 E., Volusia County, Hydrologic Unit 03080201 , on left bank $50 \mathrm{ft}$ downstream from bridge on State Highway 40A, $1.8 \mathrm{mi}$ north of Samsula, $8 \mathrm{mi}$ west of New Symrna, $10 \mathrm{mi}$ upstream from Turnbull Bay, and $13 \mathrm{mi}$ upstream from mouth.

DRAINAGE AREA. $--33.4 \mathrm{mi}^{2}$.

PERIOD OF RECORD ANALYZED.--July 1951 to June 1977.

\begin{tabular}{|c|c|c|c|c|c|c|c|c|}
\hline $\begin{array}{l}\text { Recurrence } \\
\text { interval }\end{array}$ & MAGNITUDE & $\begin{array}{l}\text { fort av } \\
\text { for in }\end{array}$ & $\begin{array}{l}\text { age } f \text {. } \\
\text { cated }\end{array}$ & $\begin{array}{l}\text { ANNUAL } \\
\text { ow, in } \\
\text { number }\end{array}$ & $\begin{array}{l}\text { LOW FL } \\
\text { cubic } \\
\text { of cons }\end{array}$ & $\begin{array}{l}\text { NS } \\
\text { eet per } \\
\text { ecutive }\end{array}$ & $\begin{array}{l}\text { secon } \\
\text { e days }\end{array}$ & \\
\hline in years & 1 & 7 & 14 & 30 & 60 & 90 & 120 & 183 \\
\hline 2 & 0.54 & 0.64 & 0.70 & 0.94 & 1.5 & 2.7 & 4.4 & 8.2 \\
\hline 5 & .22 & .28 & .34 & .48 & .65 & 1.1 & 1.5 & 2.8 \\
\hline 10 & .10 & .16 & .22 & .34 & .44 & .68 & .87 & 1.5 \\
\hline 20 & $\left(\frac{*}{*}\right)$ & .10 & .15 & .26 & .32 & .44 & .53 & .90 \\
\hline 30 & 0 & .08 & .12 & .23 & .27 & .36 & .40 & .70 \\
\hline
\end{tabular}

* Less than $0.05 \mathrm{ft}^{3} / \mathrm{s}$. 
LOCATION.--Lat $28^{\circ} 04^{\prime} 42^{\prime \prime}$, long $80^{\circ} 37^{\prime} 48^{\prime \prime}$, in NE $\frac{1}{4}$ sec.4, T.28 S., R.37 E., Hydrologic Unit 03080202, on right bank $24 \mathrm{ft}$ upstream from bridge on U.S. Highway 192, $1 \frac{1}{2} \mathrm{mi}$ west of the city hall in Melbourne, Brevard County, and $2.7 \mathrm{mi}$ upstream for mouth.

DRAINAGE AREA. $--12.6 \mathrm{mi}^{2}$.

PERIOD OF RECORD ANALYZED.--July 1951 to June 1968.

Recurrence
interval
in years
2
5
10
20

MAGNITUDE AND FREQUENCY OF ANNUAL LOW FLOWS Lowest average flow, in cubic feet per second, \begin{tabular}{lccccccc}
\multicolumn{1}{c}{ for indicated number } & of consecutive days & & \\
1 & 7 & 14 & 30 & 60 & 90 & 120 & 183
\end{tabular} $\begin{array}{llllllll}4.2 & 4.9 & 5.2 & 5.8 & 6.4 & 7.0 & 7.7 & 8.9\end{array}$ $\begin{array}{llllllll}3.2 & 3.8 & 4.1 & 4.4 & 5.0 & 5.6 & 6.1 & 6.9\end{array}$

$\begin{array}{llllllll}2.7 & 3.3 & 3.5 & 3.8 & 4.4 & 5.0 & 5.4 & 6.1\end{array}$

02250000 Turkey Creek near Palm Bay, Fla.

LOCATION.--Lat $28^{\circ} 00^{\prime} 46^{\prime \prime}$, long $80^{\circ} 37^{\prime} 20^{\prime \prime}$, in SW $\frac{7}{4}$ sec.27, T.28 S., R.37 E., Hydrologic Unit 03080202, near left bank at downstream side of bridge on State Highway 507, 2.8 mi southwest of Palm Bay, Brevard County, $3.5 \mathrm{mi}$ upstream from mouth.

DRAINAGE AREA. $--95.5 \mathrm{mi}^{2}$.

PERIOD OF RECORD ANALYZED.--July 1956 to June 1968.

Recurrence
interval
in years
2
5
10
20

MAGNITUDE AND FREQUENCY OF ANNUAL LOW FLOWS

Lowest average flow, in cubic feet per second, for indicated number of consecutive days

interval

2.43 .0

3.1

3.3

3.9

$4.5 \quad 5.0$

5.5

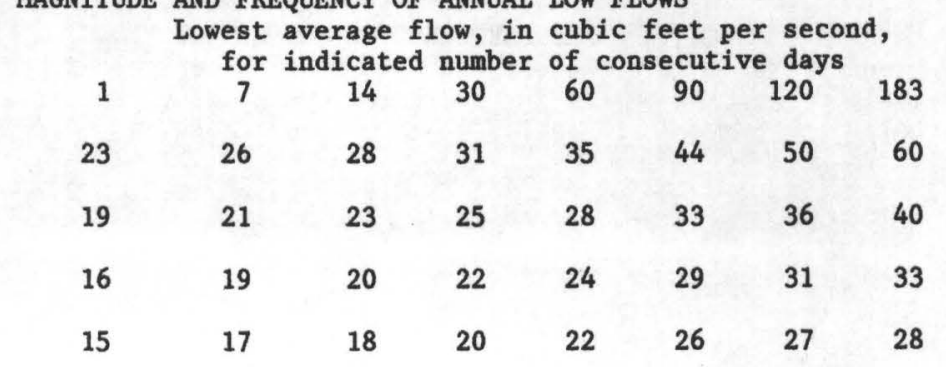




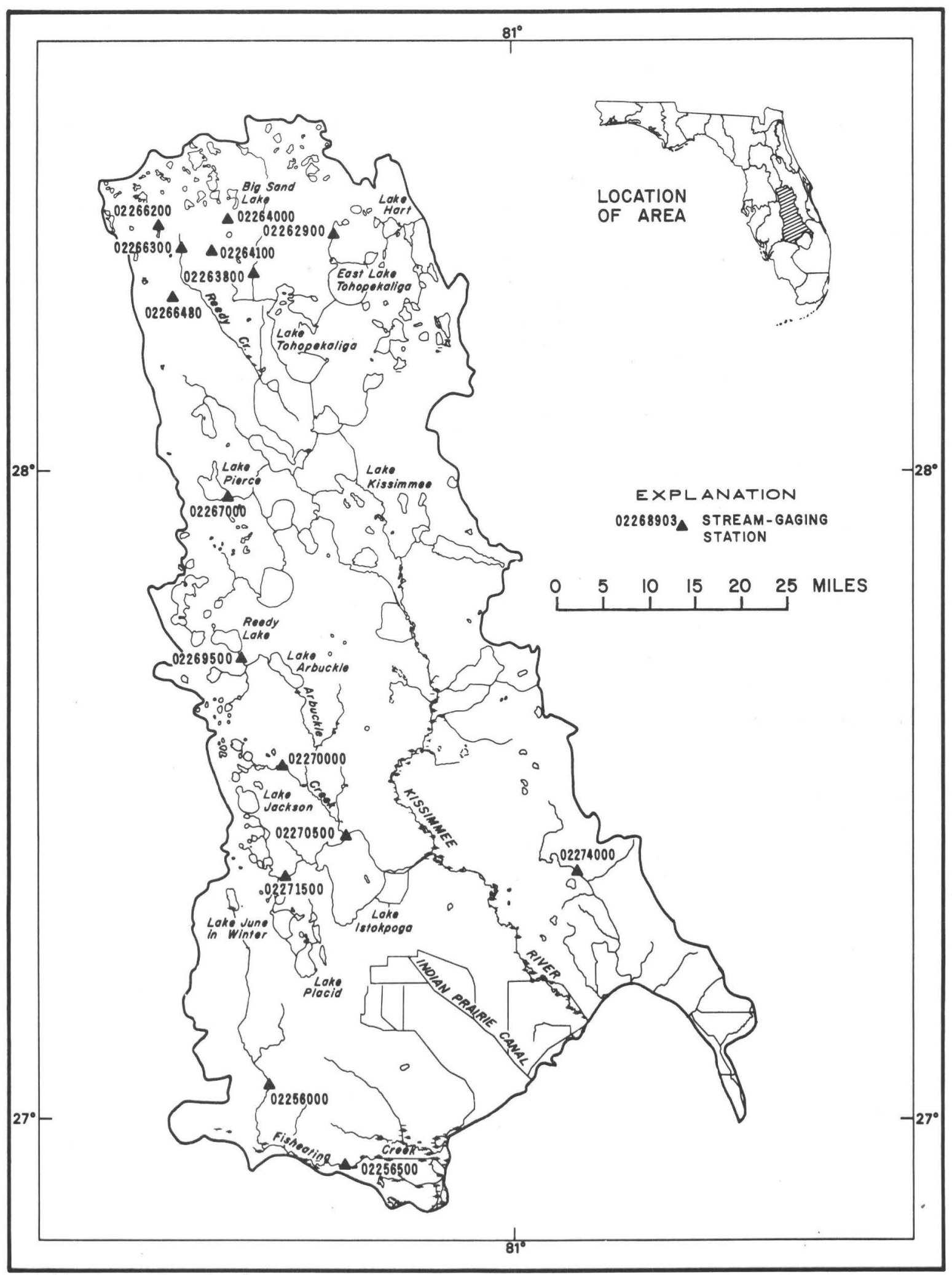

Figure 6.--Location of stream-gaging stations in the Kissimmee River, Taylor Creek, and Fisheating Creek basins. 


\section{KISSIMMEE RIVER BASIN}

02264000 Cypress Creek at Vineland, Fla.

LOCATION.--Lat $28^{\circ} 23^{\prime} 25^{\prime \prime}$, long $81^{\circ} 31^{\prime} 11^{\prime \prime}$, in NW/ sec.21, T.24 S., R.28 E., Orange County, Hydrologic Unit 03090101, at upstream side of culverts on State Highway 535, 1.0 mi west of Vineland.

DRAINAGE AREA. $--30.3 \mathrm{mi}^{2}$.

PERIOD OF RECORD ANALYZED.--July 1946 to June 1977.

REMARKS.--Some diversions by pumping above station for irrigation.

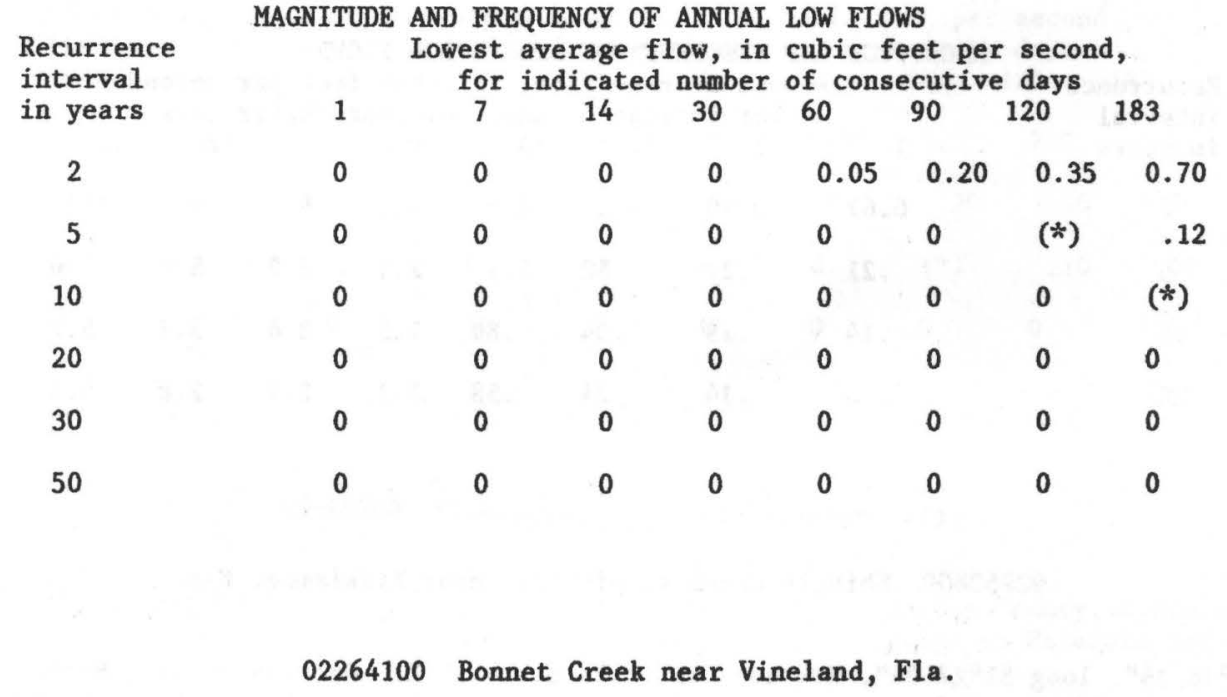

LOCATION.--Lat $28^{\circ} 19^{\prime} 58^{\prime \prime}$, long $81^{\circ} 31^{\prime} 20^{\prime \prime}$, in NW sec.9, T.25 S., R.28 E., Osceola County, Hydrologic Unit 0309101, on downstream side of bridge on State Highway 530, about 1 mi upstream from Reedy Creek Swamp and $4.5 \mathrm{mi}$ south of Vineland.

DRAINAGE AREA. --56.1 $\mathrm{mi}^{2}$.

PERIOD OF RECORD ANALYZED.--July 1966 to June 1977.

REMARKS.--Since October 1968, flow regulated by automatic gates upstream and since December 1970 , by control structure S-11-temporary $0.5 \mathrm{mi}$ downstream. Natural flow of stream affected by canals and control structures above station which divert an undertermined amount of water into the Reedy Creek basin.

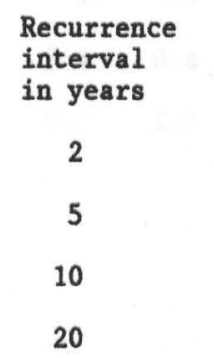

\begin{tabular}{|c|c|c|c|c|c|c|c|}
\hline TUDE & $\begin{array}{c}\text { AND FRE } \\
\text { Lowest } \\
\text { for } \\
7\end{array}$ & $\begin{array}{c}\text { QUENCY OF } \\
\text { average } \\
\text { indicated } \\
14\end{array}$ & $\begin{array}{l}\text { ANNUAL } \\
\text { low, in } \\
\text { number } \\
30\end{array}$ & $\begin{array}{l}\text { LOW FLC } \\
\text { cubic } \\
\text { of cons } \\
60\end{array}$ & $\begin{array}{l}\text { ws } \\
\text { eet pe } \\
\text { ecutiv } \\
90\end{array}$ & $\begin{array}{l}\text { secon } \\
\text { days } \\
120\end{array}$ & 183 \\
\hline 1.2 & 3.3 & 4.1 & 5.6 & 6.6 & 7.4 & 8.3 & 10 \\
\hline 0 & 1.0 & 1.3 & .88 & 1.7 & 2.3 & 3.3 & 4.7 \\
\hline 0 & 0 & $(*)$ & .20 & .30 & .60 & 1.2 & 2.5 \\
\hline 0 & 0 & 0 & (*) & .10 & .20 & .60 & 1.3 \\
\hline
\end{tabular}

* Less than $0.05 \mathrm{ft}^{3} / \mathrm{s}$. 
LOCATION.--Lat $28^{\circ} 23^{\prime} 05^{\prime \prime}$, long $81^{\circ} 37^{\prime} 00^{\prime \prime}$, in NW/ sec.21, T.24 S., R.27 E., Orange County, Hydrologic Unit 03090101 , near center of channel $12 \mathrm{ft}$ downstream from culverts on Hartzog Road, 7 mi west of Vineland.

DRAINAGE AREA. $--12.4 \mathrm{mi}^{2}$.

PERIOD OF RECORD ANALYZED.--July 1966 to June 1977.

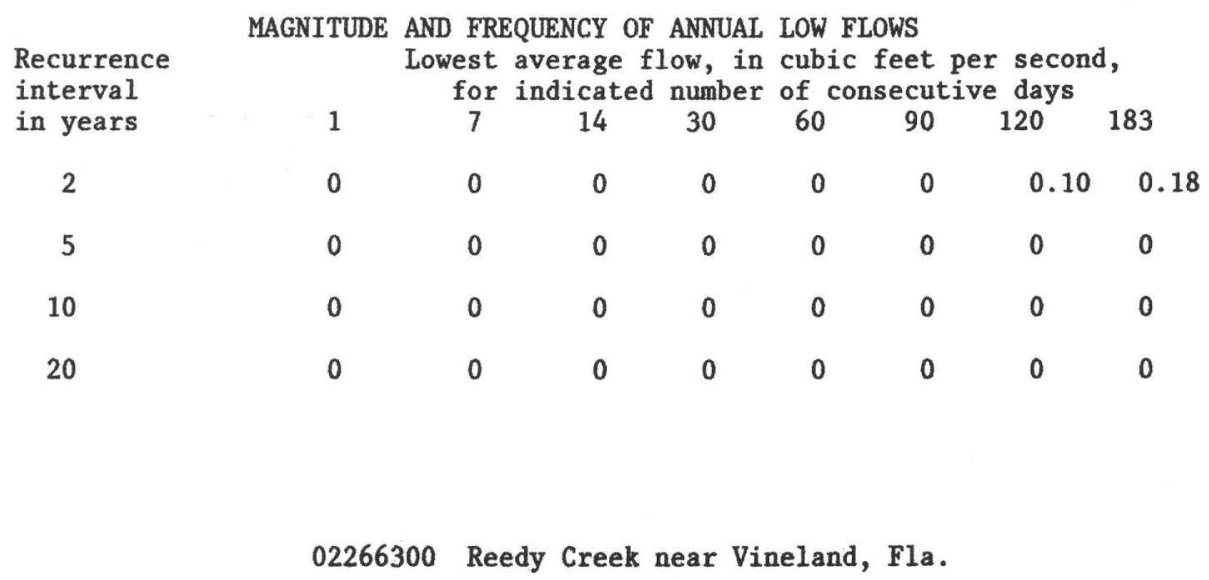

LOCATION.--Lat $28^{\circ} 19^{\prime} 57^{\prime \prime}$, long $81^{\circ} 34^{\prime} 48^{\prime \prime}$, in NE $\frac{z_{4}}{4}$ sec.11, T.25 S., R.27 E., Osceola County, Hydrologic Unit 03090101 , on downstream side of bridge on U.S. Highway 192 about 2.5 mi upstream from bridge on Interstate Highway 4, $6.5 \mathrm{mi}$ southwest of Vineland, and $28 \mathrm{mi}$ upstream from mouth.

DRAINAGE AREA. $--75 \mathrm{mi}^{2}$, approximately.

PERIOD OF RECORD ANALYZED.--July 1966 to June 1971, July 1973 to June 1977.

\begin{tabular}{|c|c|c|c|c|c|c|c|c|}
\hline $\begin{array}{l}\text { Recurrence } \\
\text { interval }\end{array}$ & MAGNITUDE & $\begin{array}{c}\text { AND FREC } \\
\text { Lowest } \\
\text { for }\end{array}$ & $\begin{array}{l}\text { QUENCY OF } \\
\text { average } f \\
\text { indicated }\end{array}$ & $\begin{array}{l}\text { ANNUAL } \\
\text { low, in } \\
\text { number }\end{array}$ & $\begin{array}{l}\text { LOW FL } \\
\text { cubic } \\
\text { of con }\end{array}$ & $\begin{array}{l}\text { WS } \\
\text { eet pe } \\
\text { ecutiv }\end{array}$ & $\begin{array}{l}r \text { secor } \\
\text { e days }\end{array}$ & d, \\
\hline in years & 1 & 7 & 14 & 30 & 60 & 90 & 120 & 183 \\
\hline 2 & 4.4 & 5.2 & 5.8 & 7.8 & 8.5 & 9.0 & 9.5 & 10 \\
\hline 5 & 0 & 0 & 0 & $(*)$ & .70 & 2.0 & 3.7 & 5.0 \\
\hline 10 & 0 & 0 & 0 & 0 & 0 & $(*)$ & 1.5 & 3.0 \\
\hline
\end{tabular}

* Less than $0.05 \mathrm{ft}^{3} / \mathrm{s}$. 
LOCATION.--Lat $28^{\circ} 16^{\prime} 15^{\prime \prime}$, long $81^{\circ} 35^{\prime} 28^{\prime \prime}$, in NW/ sec.35, T.25 S., R.26 E., Osceola County, Hydrologic Unit 03090101, at downstream side of culverts on State Highway 545, 2.0 mi upstream from mouth and 2.5 mi northwest of Loughman.

DRAINAGE AREA. $--23 \mathrm{mi}^{2}$, approximately.

PERIOD OF RECORD ANALYZED.--July 1969 to June 1977.

\begin{tabular}{|c|c|c|c|c|c|c|c|c|}
\hline $\begin{array}{l}\text { Recurrence } \\
\text { interval }\end{array}$ & MAGNITUDE & $\begin{array}{l}\text { FREQU } \\
\text { vest av } \\
\text { for in }\end{array}$ & $\begin{array}{l}\text { ICY OF } \\
\text { age f } \\
\text { cated }\end{array}$ & $\begin{array}{l}\text { ANNUAL } \\
\text { ow, in } \\
\text { number }\end{array}$ & $\begin{array}{l}\text { LOW FL } \\
\text { cubic } \\
\text { of con }\end{array}$ & $\begin{array}{l}\text { WS } \\
\text { eet pe } \\
\text { ecutiv }\end{array}$ & $\begin{array}{l}\text { r secon } \\
\text { e days }\end{array}$ & \\
\hline in years & 1 & 7 & 14 & 30 & 60 & 90 & 120 & 183 \\
\hline 2 & 0.83 & 0.97 & 1.1 & 1.2 & 1.5 & 1.8 & 2.3 & 3.7 \\
\hline 5 & .56 & .65 & .74 & .88 & 1.0 & 1.2 & 1.6 & 2.3 \\
\hline 10 & .47 & .54 & .61 & .75 & .85 & 1.1 & 1.4 & 1.9 \\
\hline
\end{tabular}

02267000 Catfish Creek near Lake Wales, Fla.

LOCATION.--Lat $27^{\circ} 57^{\prime} 40^{\prime \prime}$, long $81^{\circ} 29^{\prime} 48^{\prime \prime}$, in sec.14, T.29 S., R.28 E., Polk County, Hydrologic Unit 0309101, on left bank, 0.2 mi downstream from Lake Pierce, 7 mi northeast of city of Lake Wales, and 9.3 mi upstream from mouth.

DRAINAGE AREA. $--58.9 \mathrm{mi}^{2}$.

PERIOD OF RECORD ANALYZED.--July 1948 to June 1977.

\begin{tabular}{|c|c|c|c|c|c|c|c|c|}
\hline $\begin{array}{l}\text { Recurrence } \\
\text { interval }\end{array}$ & MAGNITUDE & $\begin{array}{l}\text { ND FRE } \\
\text { owest } \\
\text { for }\end{array}$ & $\begin{array}{l}\text { NCY OF } \\
\text { rage f } \\
\text { icated }\end{array}$ & $\begin{array}{l}\text { ANNUAL } \\
\text { ow, in } \\
\text { number }\end{array}$ & $\begin{array}{l}\text { LOW FL } \\
\text { cubic } \\
\text { of con }\end{array}$ & $\begin{array}{l}\text { WS } \\
\text { eet pe } \\
\text { ecuti }\end{array}$ & $\begin{array}{l}\text { r sec } \\
\text { e day }\end{array}$ & \\
\hline in years & 1 & 7 & 14 & 30 & 60 & 90 & 120 & 183 \\
\hline 2 & 14 & 15 & 16 & 18 & 20 & 23 & 23 & 31 \\
\hline 5 & 6.6 & 7.3 & 7.9 & 9.1 & 11 & 14 & 17 & 22 \\
\hline 10 & 4.3 & 4.8 & 5.2 & 6.1 & 8.1 & 11 & 14 & 18 \\
\hline 20 & 2.9 & 3.2 & 3.6 & 4.2 & 6.1 & 8.7 & 11 & 16 \\
\hline 30 & 2.3 & 2.6 & 3.0 & 3.5 & 5.1 & 7.7 & 10 & 15 \\
\hline
\end{tabular}


LOCATION.--Lat $27^{\circ} 43^{\prime} 1^{\prime \prime}$, long $81^{\circ} 28^{\prime} 40^{\prime \prime}$, in SW sec.1, T.32 S., R.28 E., Polk County, Hydrologic Unit 03090101, on left bank $15 \mathrm{ft}$ upstream from highway bridge, $100 \mathrm{ft}$ downstream from Reedy Lake, $1.9 \mathrm{mi}$ upstream from mouth, and $3.5 \mathrm{mi}$ southeast of Frostproof.

DRAINAGE AREA. $--60.9 \mathrm{mi}^{2}$.

PERIOD OF RECORD ANALYZED.--July 1947 to June 1971.

\begin{tabular}{|c|c|c|c|c|c|c|c|c|}
\hline $\begin{array}{l}\text { Recurrence } \\
\text { interval }\end{array}$ & MAGNITUDE & $\begin{array}{l}\text { ID FREQ } \\
\text { west a } \\
\text { for i }\end{array}$ & $\begin{array}{l}\text { UENCY OF } \\
\text { verage } f] \\
\text { ndicated }\end{array}$ & $\begin{array}{l}\text { ANNUAL } \\
\text { low, in } \\
\text { number }\end{array}$ & $\begin{array}{l}\text { LOW FL } \\
\text { cubic } \\
\text { of con }\end{array}$ & $\begin{array}{l}\text { WS } \\
\text { eet pe } \\
\text { ecutiv }\end{array}$ & $\begin{array}{l}\text { r secor } \\
\text { e days }\end{array}$ & \\
\hline in years & 1 & 7 & 14 & 30 & 60 & 90 & $120^{\circ}$ & 183 \\
\hline 2 & 8.5 & 10.5 & 12 & 13 & 16 & 20 & 22 & 25 \\
\hline 5 & .80 & 1.3 & 2.0 & 2.7 & 5.0 & 7.2 & 10 & 14 \\
\hline 10 & .15 & .35 & .40 & .65 & 1.6 & 3.0 & 6.0 & 9.9 \\
\hline 20 & (*) & (*) & .06 & .08 & .55 & 1.5 & 3.8 & 7.3 \\
\hline 30 & 0 & 0 & $(*)$ & $(*)$ & .30 & .90 & 2.8 & 6.0 \\
\hline
\end{tabular}

02270000 Carter Creek near Sebring, Fla.

LOCATION.--Lat $27^{\circ} 32^{\prime} 00^{\prime \prime}$, long $81^{\circ} 23^{\prime} 25^{\prime \prime}$, in SE光 sec.11, T.34 S., R.29 E., Hydrologic Unit 03090101, at left bank $1,100 \mathrm{ft}$ upstream from bridge on county road, $2 \frac{1}{2}$ mi upstream from Arbuckle Creek, 4 mi downstream from Bonnet

Lake, and $4 \frac{1}{4} \mathrm{mi}$ northeast of Sebring, Highlands County. Prior to Oct. 1, 1963, at site 1,100 ft downstream.

DRAINAGE AREA. $--38.8 \mathrm{mi}^{2}$.

PERIOD OF RECORD ANALYZED.--July 1955 to June 1966.

REMARKS.--Regulation by Bonnet Lake control above station.

\begin{tabular}{|c|c|c|c|c|c|c|c|c|}
\hline $\begin{array}{l}\text { Recurrence } \\
\text { interval }\end{array}$ & MAGNITUDE & $\begin{array}{c}\text { AND FRE } \\
\text { Lowest } \\
\text { for }\end{array}$ & $\begin{array}{l}\text { TCY or } \\
\text { cage } \\
\text { icated }\end{array}$ & $\begin{array}{l}\text { ANNUAL } \\
\text { Low, in } \\
\text { number }\end{array}$ & $\begin{array}{l}\text { LOW FI } \\
\text { cubic } \\
\text { of con }\end{array}$ & $\begin{array}{l}\text { WS } \\
\text { eet } p \\
\text { ecuti }\end{array}$ & $\begin{array}{l}\text { I secor } \\
\text { e days }\end{array}$ & \\
\hline in years & 1 & 7 & 14 & 30 & 60 & 90 & 120 & 183 \\
\hline 2 & 7.3 & 8.7 & 9.8 & 11 & 15 & 17 & 18 & 20 \\
\hline 5 & 4.2 & 5.1 & 5.8 & 7.1 & 9.6 & 11 & 11 & 13 \\
\hline 10 & 3.1 & 3.9 & 4.4 & 5.6 & 7.7 & 8.5 & 9.0 & 10 \\
\hline 20 & 2.4 & 3.1 & 3.5 & 4.6 & 6.5 & 7.0 & 7.4 & 8.1 \\
\hline
\end{tabular}

* Less than $0.05 \mathrm{ft}^{3} / \mathrm{s}$. 
LOCATION.--Lat $27^{\circ} 26^{\prime} 32^{\prime \prime}$, long $81^{\circ} 17^{\prime} 51^{\prime \prime}$, in SE $\frac{1}{4}$ sec.11, T.35 S., R.30 E., Highlands County, Hydrologic Unit 03090101, on right bank $20 \mathrm{ft}$ downstream from bridge on U.S. Highway 98, $1.3 \mathrm{mi}$ upstream from mouth, and 7 mi east of De Soto City.

DRAINAGE AREA.--379 $\mathrm{mi}^{2}$, excludes area drained by Lake Weohyakapka and includes area drained by Lake Sebring. PERIOD OF RECORD ANALYZED.--July 1939 to June 1977.

REMARKS.--Records include small diversions into Lake Arbuckle from Lake Weohyakapka through Blue Jordan Swamp.

\begin{tabular}{|c|c|c|c|c|c|c|c|c|}
\hline $\begin{array}{l}\text { Recurrence } \\
\text { interval }\end{array}$ & MAGNITUDE & $\begin{array}{l}\text { D FRE } \\
\text { west } \\
\text { for }\end{array}$ & $\begin{array}{l}\text { CY OF } \\
\text { rage f } \\
\text { icated }\end{array}$ & $\begin{array}{l}\text { ANNUAL } \\
\text { ow, in } \\
\text { number }\end{array}$ & $\begin{array}{l}\text { LOW FI } \\
\text { cubic } \\
\text { of cor }\end{array}$ & $\begin{array}{l}\text { WS } \\
\text { eet } \\
\text { ecut }\end{array}$ & $\begin{array}{l}\text { r se } \\
\text { e da }\end{array}$ & \\
\hline in years & 1 & 7 & 14 & 30 & 60 & 90 & 120 & 183 \\
\hline 2 & 31 & 36 & 41 & 51 & 66 & 84 & 98 & 127 \\
\hline 5 & 9.0 & 13 & 15 & 21 & 29 & 40 & 49 & 66 \\
\hline 10 & 4.1 & 6.7 & 7.6 & 13 & 18 & 26 & 33 & 46 \\
\hline 20 & 7.0 & 3.8 & 4.2 & 7.9 & 11 & 18 & 24 & 34 \\
\hline 30 & 1.4 & 2.8 & 3.0 & 6.2 & 8.0 & 14 & 20 & 29 \\
\hline 50 & .84 & 1.9 & 2.2 & 4.5 & 6.4 & 11 & 17 & 24 \\
\hline
\end{tabular}

02271500 Josephine Creek near De Soto City, Fla.

LOCATION.--Lat $27^{\circ} 22^{\prime} 26^{\prime \prime}$, long $81^{\circ} 23^{\prime} 37^{\prime \prime}$, in SE $\frac{3}{4}$ sec.2, T.36 S., R.29 E., Highlands County, Hydrologic Unit 03090101 , on left bank $320 \mathrm{ft}$ downstream from bridge on State Highway $17,1.0 \mathrm{mi}$ downstream from Jack Creek, 4 mi south of De Soto City, and 4.9 mi upstream from mouth.

DRAINAGE AREA.--109 $\mathrm{mi}^{2}$, includes area drained by Lake Sebring.

PERIOD OF RECORD ANALYZED.--July 1947 to June 1975.

REMARKS.--Diversions for irrigation of citrus groves above station during dry periods.

\begin{tabular}{|c|c|c|c|c|c|c|c|c|}
\hline $\begin{array}{l}\text { Recurrence } \\
\text { interval }\end{array}$ & MAGNITUDE & $\begin{array}{l}\text { D FREQ } \\
\text { west a } \\
\text { for } i\end{array}$ & $\begin{array}{l}\text { ICY a } \\
\text { cage } \\
\text { icated }\end{array}$ & $\begin{array}{l}\text { ANNUAL } \\
\text { ow, in } \\
\text { number }\end{array}$ & $\begin{array}{l}\text { LOW F. } \\
\text { cubic } \\
\text { of col }\end{array}$ & $\begin{array}{l}\text { WS } \\
\text { eet p } \\
\text { ecut } i\end{array}$ & $\begin{array}{l}r \text { secor } \\
\text { e days }\end{array}$ & \\
\hline in years & 1 & 7 & 14 & 30 & 60 & 90 & $120^{\circ}$ & 183 \\
\hline 2 & 6.4 & 7.4 & 8.2 & 10 & 13 & 17 & 21 & 29 \\
\hline 5 & 2.5 & 3.0 & 3.4 & 4.0 & 5.3 & 6.9 & 8.9 & 13 \\
\hline 10 & 1.5 & 1.9 & 2.1 & 2.5 & 3.3 & 4.3 & 5.6 & 8.6 \\
\hline 20 & .98 & 1.2 & 1.4 & 1.6 & 2.2 & 2.8 & 3.8 & 6.0 \\
\hline 30 & .77 & 1.0 & 1.1 & 1.3 & 1.7 & 2.3 & 3.1 & 4.9 \\
\hline
\end{tabular}


LOCATION.--Lat $27^{\circ} 23^{\prime} 39^{\prime \prime}$, long $80^{\circ} 53^{\prime} 44^{\prime \prime}$, in SE $\frac{3}{4}$ sec.26, T.35 S., R.34 E., Okeechobee County, Hydrologic Unit 03090102, near center of channel on downstream side of bridge on State Highway 68 , $800 \mathrm{ft}$ upstream from control structure 3, $0.8 \mathrm{mi}$ downstream from small tributary, $8.5 \mathrm{mi}$ east of Basinger, and $17 \mathrm{mi}$ upstream from mouth.

DRAINAGE AREA. $--15.7 \mathrm{mi}^{2}$.

PERIOD OF RECORD ANALYZED.--July 1965 to June 1977.

REMARKS.--Some diversion during low flow for irrigation. Flow regulated at station by operation of control structure 3 since February 1965.

\section{Recurrence interval}

in years

2

5

10

20

MAGNITUDE AND FREQUENCY OF ANNUAL LOW FLOWS

Lowest average flow, in cubic feet per second, for indicated number of consecutive days

1

$7 \quad 14$

$14 \quad 30$

60

90

120

183

0

0

0

0.05

0.65

0.72

1.2

1.5

0

0

0

(*)

.15

.25

.47

0

0

0

0

0

(*)

.10

.33

0

0

0

00

.01

(*) .20

* Less than $0.05 \mathrm{ft}^{3} / \mathrm{s}$. 


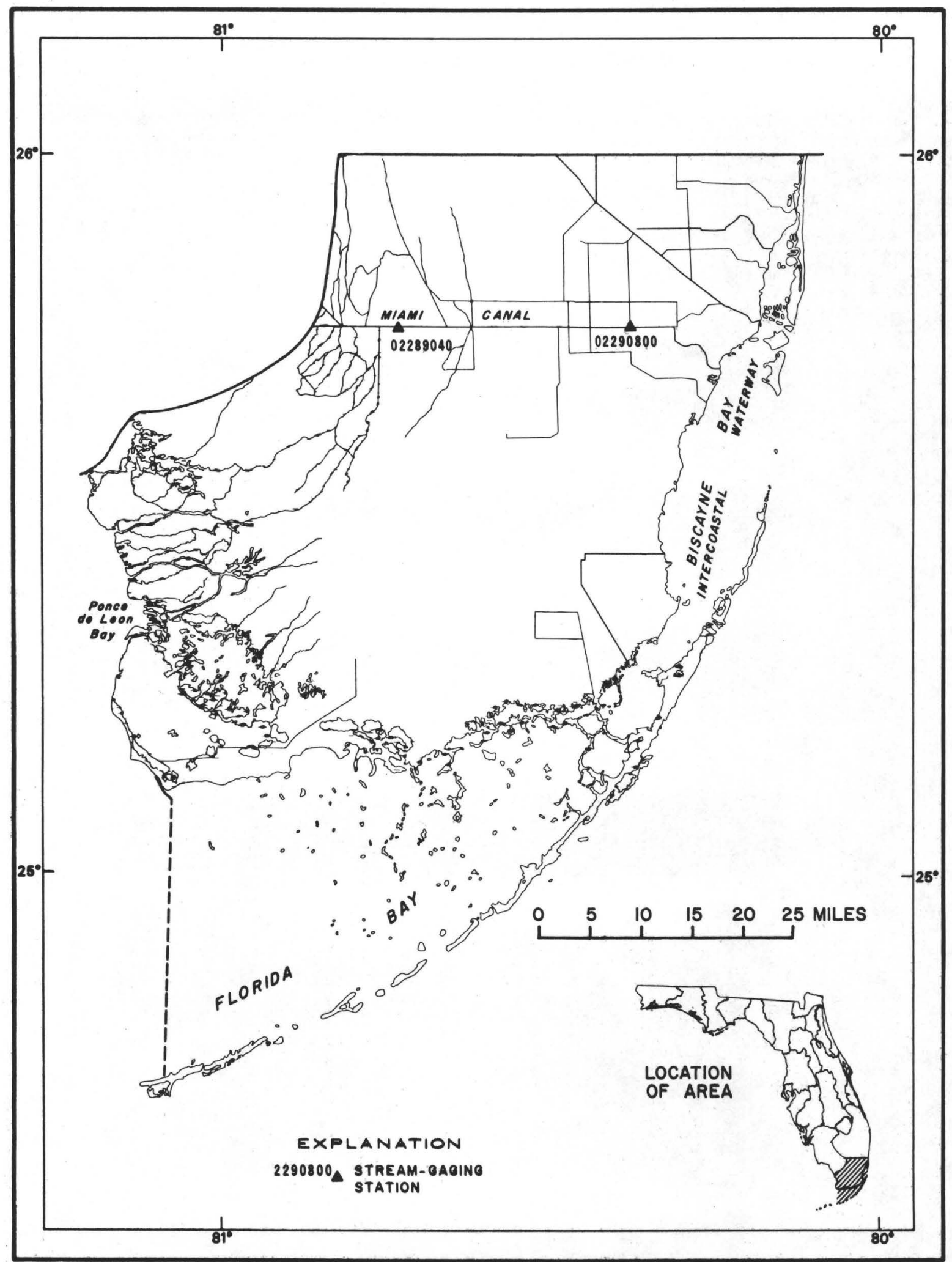

Figure 7.--Location of stream-gaging stations in the Everglades and southeastern coastal area. 


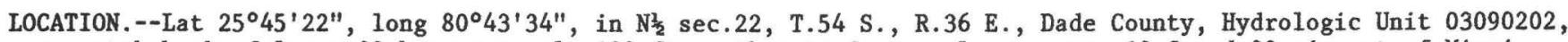
on south bank of levee 29 borrow canal, $100 \mathrm{ft}$ northwest of control structure $12-\mathrm{C}$ and $33 \mathrm{mi}$ west of Miami.

DRAINAGE AREA.--Indeterminate.

PERIOD OF RECORD ANALYZED.--July 1964 to June 1978.

REMARKS.--Discharge is the total discharge through the S-12 structure A, B, C, and D from Conservation Area 3-A.

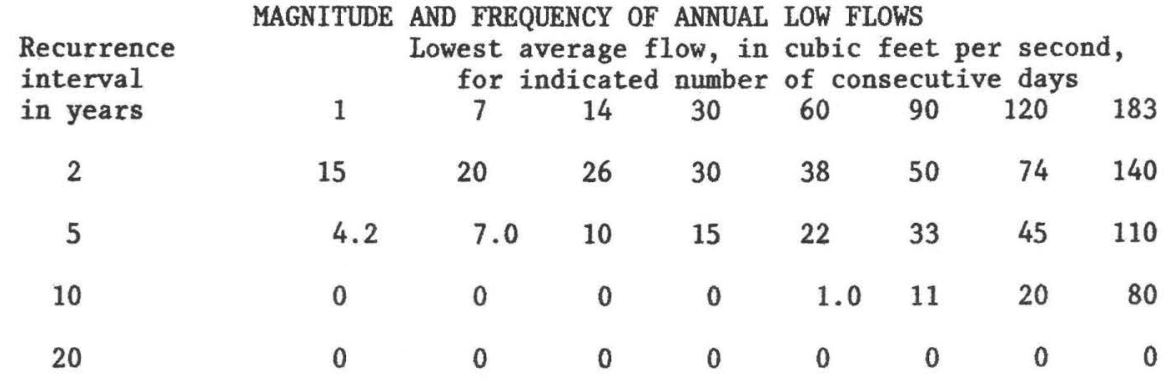

02289060 Tamiami Canal Outlets, Levee 30 to Levee 67A, near Miami, Fla.

LOCATION.--Lat $25^{\circ} 45^{\prime} 40^{\prime \prime}$, long $80^{\circ} 33^{\prime} 40^{\prime \prime}$, in SE $\frac{3}{4}$ sec.6, T.54 S., R.38 E., Dade County, Hydrologic Unit 03090202, on south bank, $50 \mathrm{ft}$ west of bridge 53 on U.S. Highway 41 and $22.8 \mathrm{mi}$ west of Miami.

DRAINAGE AREA.--Indeterminate.

PERIOD OF RECORD ANALYZED.--July 1964 to June 1977.

REMARKS.--Discharge consists entirely of seepage through levee 29 from Conservation Area $3 B$ as represented by flow through all the outlets of Tamiami Canal from levee 30 to levee 67A.

Recurrence
interval
in years
2
5
10
20

MAGNITUDE AND FREQUENCY OF ANNUAL LOW FLOWS

Lowest average flow, in cubic feet per second, for indicated number of consecutive days

$$
\begin{aligned}
& \text { interval } \\
& \text { in years }
\end{aligned}
$$

MAGNITUDE AND FREQUENCY OF ANNUAL LOW FLOWS
Lowest average flow, in cubic feet per second,
for indicated number of consecutive days




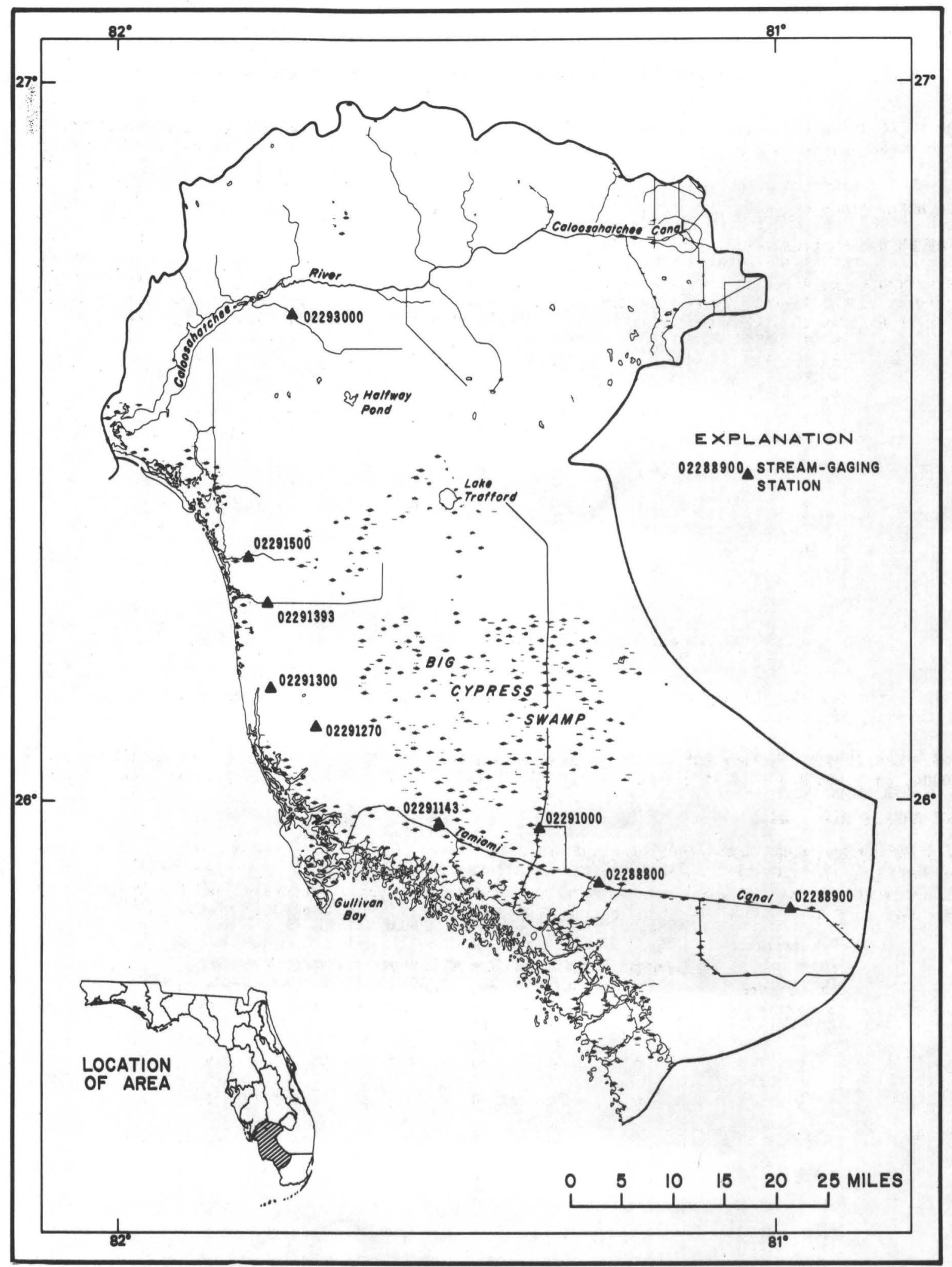

Figure 8.--Location of stream-gaging stations in the Big Cypress Swamp and southwestern coastal area, including the Caloosahatchee River. 
LOCATION.--Lat $25^{\circ} 53^{\prime} 10^{\prime \prime}$, long $81^{\circ} 15^{\prime} 30^{\prime \prime}$, in NW/ sec.6, T.53 S., R.31 E., Collier County, Hydrologic Unit 03090204 , on downstream side of bridge' 84 on U.S. Highway 41,7 mi east of Carnestown, and 10 mi west of Monroe.

DRAINAGE AREA.--Indeterminate.

PERIOD OF RECORD ANALYZED.--July 1961 to July 1977.

REMARKS.--Figures of discharge consist of runoff from Big Cypress Watershed as represented by flow through all the outlets of the Tamiami Canal from Monroe, $55 \mathrm{mi}$ west of Miami, to a point 1 mi east of the intersection with State Highway 29 at Carnestown. Flow at westernmost outlets slightly affected by tide.

\begin{tabular}{|c|}
\hline $\begin{array}{l}\text { Recurrence } \\
\text { interval } \\
\text { in years }\end{array}$ \\
\hline 2 \\
\hline 5 \\
\hline 10 \\
\hline \\
\hline
\end{tabular}

MAGNITUDE AND FREQUENCY OF ANNUAL LOW FLOWS

Lowest average flow, in cubic feet per second, for indicated number of consecutive days

\section{interval}

Lowest average flow, in cubic feet per second,
for indicated number

02288900 Tamiami Canal Outlets, 40-Mile Bend to Monroe, Fla.

LOCATION.--Lat $25^{\circ} 51^{\prime} 05^{\prime \prime}$, long $80^{\circ} 58^{\prime} 50^{\prime \prime}$, in SW sec.13, T.53 S., R.33 E., Collier County, Hydrologic Unit 03090204, on south bank, $25 \mathrm{ft}$ east of bridge 105 on U.S. Highway 41 and 54 mi west of Miami, Dade County.

DRAINAGE AREA.--Indeterminate.

PERIOD OF RECORD ANALYZED.--July 1964 to June 1977.

REMARKS.--Figures of daily discharge consist of runoff from Big Cypress Watershed and the Everglades as represented by flow through all 29 bridges from bridge 28 to 22 and bridge 117 to 96.

\begin{tabular}{ccccccccccc}
$\begin{array}{l}\text { Recurrence } \\
\text { interval } \\
\text { in years }\end{array}$ & 1 & \multicolumn{7}{c}{$\begin{array}{c}\text { Lowest average flow, in cubic feet per second, } \\
\text { for indicated number of consecutive days }\end{array}$} \\
2 & 0 & 7 & 14 & 30 & 60 & 90 & 120 & 183 \\
5 & 0 & 0 & 0.17 & 0.75 & 2.2 & 5.7 & 17 \\
10 & 0 & 0 & 0 & 0 & 0 & 0.12 & 1.3 & 6.7 \\
20 & 0 & 0 & 0 & 0 & $(*)$ & .62 & 3.7 \\
& 0 & 0 & 0 & 0 & 0 & $(*)$ & .36 & 2.2
\end{tabular}

MAGNITUDE AND FREQUENCY OF ANNUAL LOW FLOWS

Lowest average flow, in cubic feet per second, $\begin{array}{ccccccc}\text { for } & \text { indicated number of consecutive days } & & \\ 7 & 14 & 30 & 60 & 90 & 120 & 183\end{array}$

\footnotetext{
* Less than $0.05 \mathrm{ft}^{3} / \mathrm{s}$.
} 
LOCATION.--Lat $25^{\circ} 57^{\prime} 28^{\prime \prime}$, long $81^{\circ} 21^{\prime} 19^{\prime \prime}$, in NW/ sec.7, T.52 S., R.30 E., Collier County, Hydrologic Unit 03090204, on right bank $40 \mathrm{ft}$ upstream from control structure, $0.7 \mathrm{mi}$ north of Copeland, $7 \mathrm{mi}$ north of town of Everglades, and $7.5 \mathrm{mi}$ upstream from mouth.

DRAINAGE AREA.--Indeterminate.

PERIOD OF RECORD ANALYZED.--July 1952 to June 1977.

REMARKS.--Flow regulated by operation of control structure at, above, and below station, and is occasionally affected by tide.

\begin{tabular}{|c|c|c|c|c|c|c|c|c|}
\hline $\begin{array}{l}\text { Recurrence } \\
\text { interval }\end{array}$ & MAGNITUDE & $\begin{array}{l}\text { FREQU } \\
\text { vest av } \\
\text { for in }\end{array}$ & $\begin{array}{l}\text { ICY OF } \\
\text { age } f \\
\text { cated }\end{array}$ & $\begin{array}{l}\text { ANNUAL } \\
\text { ow, in } \\
\text { number }\end{array}$ & $\begin{array}{l}\text { LOW FLC } \\
\text { cubic } \\
\text { of cons }\end{array}$ & $\begin{array}{l}\text { WS } \\
\text { eet pe } \\
\text { ecuti }\end{array}$ & $\begin{array}{l}\text { r secon } \\
\text { e days }\end{array}$ & \\
\hline in years & 1 & 7 & 14 & 30 & 60 & 90 & 120 & 183 \\
\hline 2 & 7.0 & 8.0 & 8.6 & 11 & 16 & 21 & 28 & 42 \\
\hline 5 & 1.8 & 2.2 & 2.5 & 3.2 & 3.9 & 7.5 & 11 & 20 \\
\hline 10 & .21 & .30 & .50 & .70 & 1.6 & 4.2 & 6.0 & 12 \\
\hline 20 & $(*)$ & .05 & .08 & .10 & .72 & 2.2 & 3.5 & 7.5 \\
\hline 30 & 0 & 0 & 0 & (*) & .46 & 1.4 & 2.4 & 5.2 \\
\hline
\end{tabular}

02291143 Faka Union Canal near Copeland, Fla.

LOCATION.--Lat $25^{\circ} 57^{\prime} 59^{\prime \prime}$, long $81^{\circ} 30^{\prime} 23^{\prime \prime}$, in SW sec.3, T.52 S., R.28 E., Collier County, Hydrologic Unit 03090204 , on left bank, $0.5 \mathrm{mi}$ from U.S. Highway 41 , and $9.3 \mathrm{mi}$ west of Copeland.

DRAINAGE AREA.--Indeterminate.

PERIOD OF RECORD ANALYZED.--July 1970 to June 1977.

\begin{tabular}{|c|c|c|c|c|c|c|c|c|}
\hline $\begin{array}{l}\text { Recurrence } \\
\text { interva1 }\end{array}$ & MAGNITUDE & $\begin{array}{c}\text { AND FRE } \\
\text { Lowest } \\
\text { for }\end{array}$ & $\begin{array}{l}\text { EQUENCY OF } \\
\text { average } \\
\text { indicated }\end{array}$ & $\begin{array}{l}\text { ANNUAL } \\
\text { low, in } \\
\text { number }\end{array}$ & $\begin{array}{l}\text { LOW } \\
\text { cubi } \\
\text { of } c\end{array}$ & $\begin{array}{l}\text { FLOWS } \\
\text { c feet per } \\
\text { onsecutive }\end{array}$ & $\begin{array}{l}\text { second } \\
\text { days }\end{array}$ & \\
\hline in years & 1 & 7 & 14 & 30 & 60 & 90 & $120^{\circ}$ & 183 \\
\hline 2 & 0 & 0 & 0 & 0 & 0 & 2.3 & 5.2 & 26 \\
\hline 5 & 0 & 0 & 0 & 0 & 0 & .15 & .40 & 5.4 \\
\hline 10 & 0 & 0 & 0 & 0 & 0 & 0 & 0 & 2.6 \\
\hline
\end{tabular}

* Less than $0.05 \mathrm{ft}^{3} / \mathrm{s}$. 
LOCATION.--Lat $26^{\circ} 05^{\prime} 59^{\prime \prime}$, long $81^{\circ} 4^{\prime} 1^{\prime \prime}$, in SW sec.23, T.50 S., R.26 E., Collier County, Hydrologic Unit 030902024, on right bank $5 \mathrm{ft}$ downstream from private bridge, $3.8 \mathrm{mi}$ south of Everglades Parkway and $7 \mathrm{mi}$ southeast of Naples.

DRAINAGE AREA.--Indeterminate.

PERIOD OF RECORD ANALYZED.--July 1969 to June 1977.

\begin{tabular}{|c|c|c|c|c|c|c|c|c|}
\hline $\begin{array}{l}\text { Recurrence } \\
\text { interval }\end{array}$ & MAGNITUDE & $\begin{array}{l}\text { FREQU } \\
\text { vest av } \\
\text { for in }\end{array}$ & $\begin{array}{l}\text { JCY OF } \\
\text { cage } f \\
\text { cated }\end{array}$ & $\begin{array}{l}\text { ANNUAL } \\
\text { ow, in } \\
\text { number }\end{array}$ & $\begin{array}{l}\text { LOW FLO } \\
\text { cubic } f \\
\text { of cons }\end{array}$ & $\begin{array}{l}\text { IS } \\
\text { et pe } \\
\text { cutiv }\end{array}$ & $\begin{array}{l}\text { I secor } \\
\text { e days }\end{array}$ & \\
\hline in years & 1 & 7 & 14 & 30 & 60 & 90 & $120^{\circ}$ & 183 \\
\hline 2 & 0.22 & 0.40 & 0.50 & 0.90 & 1.4 & 2.1 & 2.8 & 5.0 \\
\hline 5 & 0 & 0 & $(\stackrel{2}{n})$ & .10 & .18 & .46 & 1.4 & 2.9 \\
\hline 10 & 0 & 0 & 0 & $(\%)$ & .05 & .17 & 1.1 & 2.2 \\
\hline
\end{tabular}

LOCATION.--Lat $26^{\circ} 10^{\prime} 01^{\prime \prime}$, long $81^{\circ} 46^{\prime} 02^{\prime \prime}$, in NE玄 sec.35, T.49 S., R.25 E., Collier County, Hydrologic Unit 03090204 , near right bank on downstream side of bridge on Airport Road, $0.5 \mathrm{mi}$ upstream from dam, $1.4 \mathrm{mi}$ upstream from Gordon River, and $1.5 \mathrm{mi}$ east of Naples city limits.

DRAINAGE AREA.--Indeterminate.

PERIOD OF RECORD ANALYZED.--July 1965 to June 1977.

\begin{tabular}{|c|c|c|c|c|c|c|c|c|}
\hline $\begin{array}{l}\text { Recurrence } \\
\text { interval }\end{array}$ & MAGNITUDE & $\begin{array}{l}\text { ND FRE } \\
\text { owest } \\
\text { for }\end{array}$ & $\begin{array}{l}\text { NCY of } \\
\text { rage fI } \\
\text { licated }\end{array}$ & $\begin{array}{l}\text { ANNUAL } \\
\text { ow, in } \\
\text { number }\end{array}$ & $\begin{array}{l}\text { LOW FL } \\
\text { cubic } \\
\text { of con }\end{array}$ & $\begin{array}{l}\text { WS } \\
\text { eet pe } \\
\text { ecuti }\end{array}$ & $\begin{array}{l}\text { secon } \\
\text { days }\end{array}$ & \\
\hline in years & 1 & 7 & 14 & 30 & 60 & 90 & 120 & 183 \\
\hline 2 & 13 & 25 & 29 & 35 & 50 & 64 & 80 & 95 \\
\hline 5 & $(\stackrel{*}{*})$ & $(\hbar)$ & .08 & .20 & 3.5 & 11 & 23 & 49 \\
\hline 10 & 0 & 0 & 0 & 0 & .85 & 4.6 & 13 & 30 \\
\hline 20 & 0 & 0 & 0 & 0 & .22 & 2.1 & 7.4 & 20 \\
\hline
\end{tabular}

* Less than $0.05 \mathrm{ft}^{3} / \mathrm{s}$. 
LOCATION.--Lat $26^{\circ} 16^{\prime} 21^{\prime \prime}$, long $81^{\circ} 45^{\prime} 53^{\prime \prime}$, in SW sec.24, T.48 S., R.25 E., Collier County, Hydrologic Unit 03090204, near center span on downstream side of Willoughby Acres bridge, 2.3 mi east of intersection of U.S. Highway 41 and State Highway 846 at Naples Park.

DRAINAGE AREA.--Indeterminate.

PERIOD OF RECORD ANALYZED.--July 1969 to June 1977.

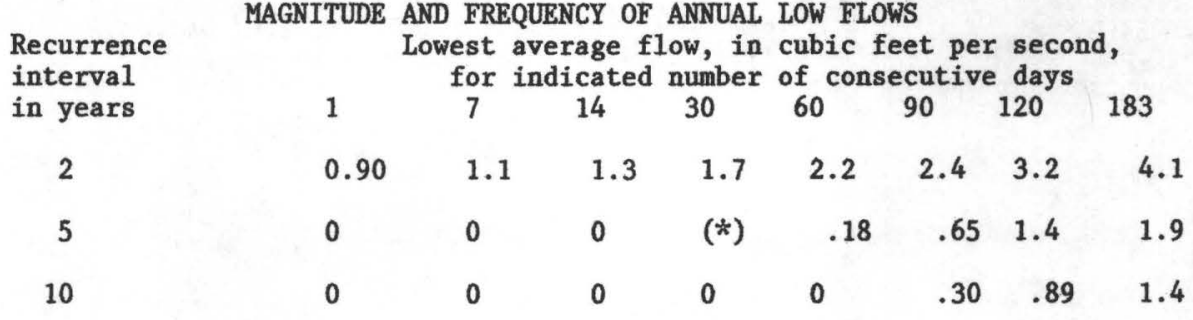

02291500 Imperial River near Bonita Springs, Fla.

LOCATION.--Lat $26^{\circ} 20^{\prime} 05^{\prime \prime}$, long $81^{\circ} 4^{\prime} 20^{\prime \prime}$, in SE $\frac{3}{4}$ sec.36, T.47 S., R.25 E., Hydrologic Unit 03090204, on right bank $1 \frac{1}{2}$ mi east of Bonita Springs, Lee County.

DRAINAGE AREA.--Indeterminate.

PERIOD OF RECORD ANALYZED.--July 1940 to June 1954.

\begin{tabular}{|c|c|c|c|c|c|c|c|c|}
\hline $\begin{array}{l}\text { Recurrence } \\
\text { interval }\end{array}$ & MAGNITUDE & $\begin{array}{l}\text { FREQ } \\
\text { west av } \\
\text { for in }\end{array}$ & $\begin{array}{l}C Y \text { OF } \\
\text { age } f 1 \\
\text { cated }\end{array}$ & $\begin{array}{l}\text { INNUAL } \\
\text { W, in } \\
\text { aumber }\end{array}$ & $\begin{array}{l}\text { LOW FLO } \\
\text { cubic } f \\
\text { of cons }\end{array}$ & $\begin{array}{l}\text { WS } \\
\text { eet per } \\
\text { ecutive }\end{array}$ & $\begin{array}{l}r \text { secono } \\
\text { e days }\end{array}$ & \\
\hline in years & 1 & 7 & 14 & 30 & 60 & 90 & 120 & 183 \\
\hline 2 & 0.75 & 0.83 & 0.90 & 0.97 & 1.1 & 1.2 & 1.3 & 1.4 \\
\hline 5 & .55 & .62 & .66 & .71 & .80 & .95 & .98 & 1.1 \\
\hline 10 & .35 & .56 & .59 & .63 & .73 & .87 & .90 & 1.0 \\
\hline 20 & 0 & .52 & .54 & .58 & .70 & .78 & .82 & .90 \\
\hline
\end{tabular}

* Less than $0.05 \mathrm{ft}^{3} / \mathrm{s}$. 
LOCATION.--Lat $26^{\circ} 40^{\prime}$, long $81^{\circ} 43^{\prime}$, in sec.9, T.44 S., R.26 E., Lee County Hydrologic Unit 03090205, $1 \frac{1}{2} \mathrm{mi}$ southeast of Buckingham and $8 \mathrm{mi}$ northeast of Fort Myers.

DRAINAGE AREA. $--83.4 \mathrm{mi}^{2}$, approximately.

PERIOD OF RECORD ANALYZED.--July 1936 to June 1946.

Recurrence

in years

2

5

10

20

MAGNITUDE AND FREQUENCY OF ANNUAL LOW FLOWS

Lowest average flow, in cubic feet per second, for indicated number of consecutive days

1

$$
7
$$

14

30

60

90

120

183

0

0

0

(*)

0.07

0.25

0.75

2.2

0

0

0

(*) (*)

(*) .52

0

0

0

0

0

(*) .20

0

0

0

0

0

0

$0 \quad .08$

* Less than $0.05 \mathrm{ft}^{3} / \mathrm{s}$. 


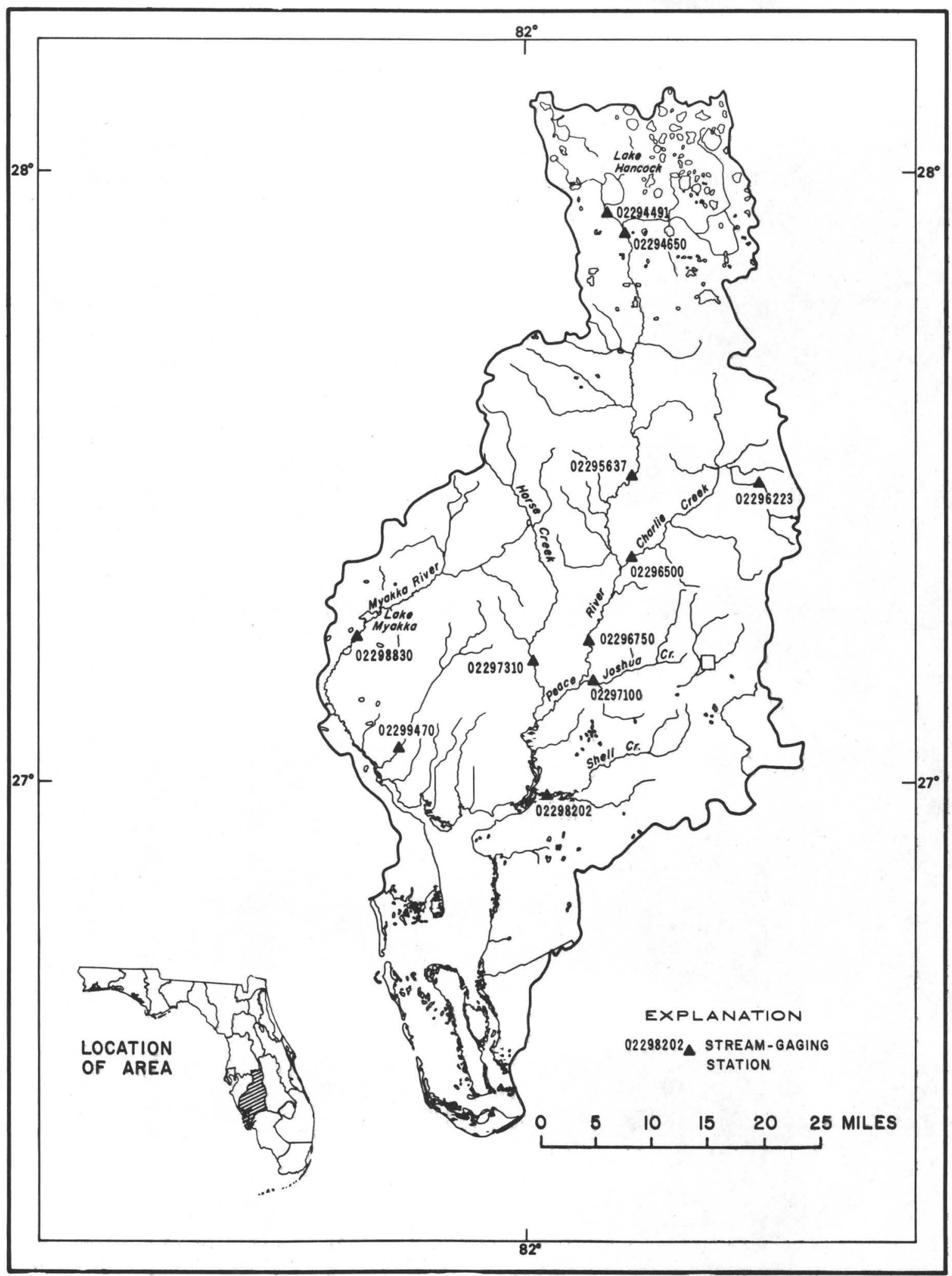

Figure 9.--Location of stream-gaging stations in the Peace and Myakka River basins. 


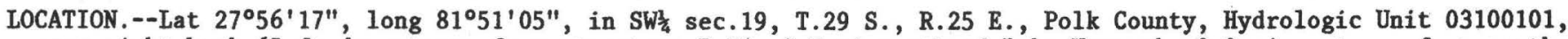
near right bank $65 \mathrm{ft}$ downstream from structure P-11, $0.7 \mathrm{mi}$ south of Lake Hancock, $2.3 \mathrm{mi}$ upstream from mouth, and $3.0 \mathrm{mi}$ north of post office in Bartow.

DRAINAGE AREA. $--135 \mathrm{mi}^{2}$.

PERIOD OF RECORD ANALYZED.--July 1964 to June 1977.

REMARKS.--Flow regulated by structure $\mathrm{P}-11$.

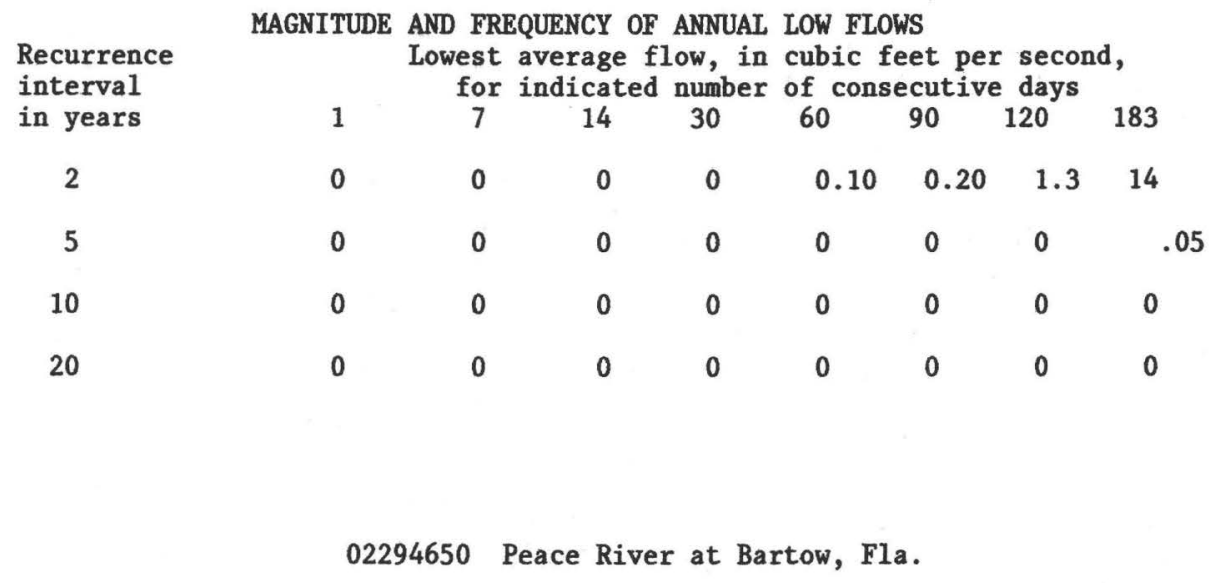

LOCATION.--Lat $27^{\circ} 54^{\prime} 07^{\prime \prime}$, long $81^{\circ} 49^{\prime} 03^{\prime \prime}$, in NE妾 sec.4, T.30 S., R.25 E., Polk County, Hydrologic Unit 03100101, near center of span on downstream side of bridge on State Highway $60,500 \mathrm{ft}$ downstream from McKinney Branch, $0.6 \mathrm{mi}$ east of Bartow, and $105 \mathrm{mi}$ upstream from mouth.

DRAINAGE AREA. $--390 \mathrm{mi}^{2}$.

PERIOD OF RECORD ANALYZED.--July 1940 to June 1977.

REMARKS.--Since 1949, records include an appreciable amount of waste water diverted from ground-water supplies into McKinney Branch by chemical plants and phosphate mines; since July 1963, some regulation by control structure P-11 on Saddle Creek.

\begin{tabular}{|c|c|c|c|c|c|c|c|c|}
\hline $\begin{array}{l}\text { Recurrence } \\
\text { interval }\end{array}$ & & $\begin{array}{l}\text { vest } \\
\text { for }\end{array}$ & $\begin{array}{l}\text { average } f \\
\text { indicated }\end{array}$ & $\begin{array}{l}\text { low, in } \\
\text { number }\end{array}$ & $\begin{array}{l}\text { cubic } \\
\text { of con }\end{array}$ & $\begin{array}{l}\text { eet } \\
\text { ecut }\end{array}$ & $\begin{array}{l}\text { r se } \\
\text { e day }\end{array}$ & \\
\hline in years & 1 & 7 & 14 & 30 & 60 & 90 & 120 & 183 \\
\hline 2 & 24 & 28 & 31 & 35 & 44 & 59 & 74 & 103 \\
\hline 5 & 9.5 & 12 & 14 & 17 & 22 & 25 & 32 & 42 \\
\hline 10 & 5.3 & 6.6 & 7.8 & 11 & 14 & 18 & 22 & 32 \\
\hline 20 & 2.0 & 3.7 & 4.5 & 7.4 & 10 & 13 & 17 & 21 \\
\hline 30 & 1.3 & 2.8 & 3.5 & 5.8 & 8.4 & 11 & 14 & 18 \\
\hline 50 & .78 & 2.0 & 2.6 & 4.8 & 7.2 & 10 & 12 & 16 \\
\hline
\end{tabular}

MAGNITUDE AND FREQUENCY OF ANNUAL LOW FLOWS

Lowest average flow, in cubic feet per second, for indicated number of consecutive days 
LOCATION.--Lat $27^{\circ} 30^{\prime} 15^{\prime \prime}$, long $81^{\circ} 48^{\prime} 04^{\prime \prime}$, in SE $\frac{1}{4}$ sec.22, T.34 S., R.25 E., Hardee County, Hydrologic Unit 03100101 , near center of span on downstream side of bridge on U.S. Highway 17, $0.8 \mathrm{mi}$ north of Zolfo Springs, and $69 \mathrm{mi}$ upstream from mouth.

DRAINAGE AREA. $--826 \mathrm{mi}^{2}$.

PERIOD OF RECORD ANALYZED.--July 1934 to June 1977.

\begin{tabular}{|c|c|c|c|c|c|c|c|c|}
\hline $\begin{array}{l}\text { Recurrence } \\
\text { interval }\end{array}$ & MAGNITL & $\begin{array}{l}\text { AND FH } \\
\text { Lowest } \\
\text { for }\end{array}$ & $\begin{array}{l}\text { QQUENCY } \\
\text { average } \\
\text { indicat }\end{array}$ & $\begin{array}{l}\text { E ANNU } \\
\text { flow, } \\
\text { d numb }\end{array}$ & $\begin{array}{l}\text { Low } \\
\text { cubi } \\
\text { of }\end{array}$ & $\begin{array}{l}\text { OWS } \\
\text { feet } \\
\text { isecut }\end{array}$ & $\begin{array}{l}\text { sec sec } \\
\text { re day }\end{array}$ & \\
\hline in years & 1 & 7 & 14 & 30 & 60 & 90 & $120^{\circ}$ & 183 \\
\hline 2 & 94 & 102 & 111 & 125 & 151 & 190 & 227 & 301 \\
\hline 5 & 54 & 64 & 73 & 80 & 101 & 115 & 140 & 160 \\
\hline 10 & 44 & 53 & 60 & 70 & 86 & 99 & 110 & 135 \\
\hline 20 & 37 & 44 & 54 & 65 & 76 & 82 & 91 & $\approx 15$ \\
\hline 30 & 34 & 40 & 49 & 61 & 70 & 76 & 82 & 107 \\
\hline 50 & 32 & 36 & 45 & 58 & 66 & 70 & 73 & 100 \\
\hline
\end{tabular}

02296223 Little Charley Bowlegs Creek near Sebring, Fla.

LOCATION.--Lat $27^{\circ} 28^{\prime} 40^{\prime \prime}$, long $81^{\circ} 33^{\prime} 2^{\prime \prime}$, in NW/ sec.31, T.34 S., R.28 E., Highlands County, Hydrologic Unit 03100101 , on right bank $160 \mathrm{ft}$ downstream from control structure, $900 \mathrm{ft}$ north of county road in Highlands Hammock State Park, 0.8 mi upstream from unnamed creek, 7.1 mi southwest of Sebring, and 7.3 mi upstream from mouth.

DRAINAGE AREA. $--41.9 \mathrm{mi}^{2}$.

PERIOD OF RECORD ANALYZED.--July 1952 to June 1977.

REMARKS.--Flow affected for short periods by operation of control structure $160 \mathrm{ft}$ upstream from station.

\begin{tabular}{|c|c|c|c|c|c|c|c|c|}
\hline $\begin{array}{l}\text { Recurrence } \\
\text { interval }\end{array}$ & MAGNITU & $\begin{array}{l}\text { AND FI } \\
\text { Lowest } \\
\text { for }\end{array}$ & $\begin{array}{l}\text { EQUENCY OF } \\
\text { average } \\
\text { indicated }\end{array}$ & $\begin{array}{l}\text { ANNUAL } \\
\text { low, in } \\
\text { number }\end{array}$ & $\begin{array}{l}\text { LOW FL } \\
\text { cubic } \\
\text { of con }\end{array}$ & $\begin{array}{l}\text { DWS } \\
\text { feet pe: } \\
\text { secutiv }\end{array}$ & $\begin{array}{l}r \text { secon } \\
\text { e days }\end{array}$ & \\
\hline in years & 1 & 7 & 14 & 30 & 60 & 90 & $120^{\circ}$ & 183 \\
\hline 2 & 0 & 0 & (*) & 0.24 & 0.50 & 2.5 & 3.7 & 7.0 \\
\hline 5 & 0 & 0 & 0 & $(*)$ & .06 & .28 & .50 & 1.0 \\
\hline 10 & 0 & 0 & 0 & 0 & $(*)$ & .13 & .24 & .45 \\
\hline 20 & 0 & 0 & 0 & 0 & $(*)$ & .06 & .12 & .27 \\
\hline 30 & 0 & 0 & 0 & 0 & 0 & (*) & .07 & .22 \\
\hline
\end{tabular}

* Less than $0.05 \mathrm{ft}^{3} / \mathrm{s}$. 
LOCATION.--Lat $27^{\circ} 22^{\prime} 29^{\prime \prime}$, Iong $81^{\circ} 47^{\prime} 48^{\prime \prime}$, in SE $\frac{3}{4}$ sec.3, T.36 S., R.25 E., Hardee County, Hydrologic Unit 03100101 , near center of span on downstream side of bridge on U.S. Highway $17,1.6$ mi north of Gardner, and 4.9 mi upstream from mouth.

DRAINAGE AREA. $--330 \mathrm{mi}^{2}$.

PERIOD OF RECORD ANALYZED.--July 1950 to June 1977.

\begin{tabular}{|c|c|c|c|c|c|c|c|c|}
\hline $\begin{array}{l}\text { Recurrence } \\
\text { interval }\end{array}$ & MAGNITUDE & $\begin{array}{l}\text { FREQ } \\
\text { est a } \\
\text { for ir }\end{array}$ & $\begin{array}{l}\text { ICY OF } \\
\text { age } f] \\
\text { cated }\end{array}$ & $\begin{array}{l}\text { ANNUAL } \\
\text { ow, in } \\
\text { number }\end{array}$ & $\begin{array}{l}\text { LOW FLC } \\
\text { cubic } \\
\text { of cons }\end{array}$ & $\begin{array}{l}\text { WS } \\
\text { eet pe } \\
\text { ecutiv }\end{array}$ & $\begin{array}{l}\text { secor } \\
\text { days }\end{array}$ & \\
\hline in years & 1 & 7 & 14 & 30 & 60 & 90 & 120 & 183 \\
\hline 2 & 2.3 & 2.8 & 3.4 & 5.1 & 11 & 20 & 29 & 75 \\
\hline 5 & .79 & 1.0 & 1.3 & 1.9 & 2.5 & 4.3 & 7.0 & 10 \\
\hline 10 & .44 & .58 & 74 & 1.0 & 1.3 & 2.2 & 4.1 & 7.0 \\
\hline 20 & .26 & .37 & .47 & .61 & .92 & 1.8 & 2.8 & 5.2 \\
\hline 30 & .20 & .30 & .38 & .44 & .68 & 1.4 & 2.1 & 4.2 \\
\hline
\end{tabular}

02296750 Peace River at Arcadia, Fla.

LOCATION.--Lat $27^{\circ} 13^{\prime} 19^{\prime \prime}$, long $81^{\circ} 52^{\prime} 34^{\prime \prime}$, in SE衣 sec.26, T.37 S., R.24 E., De Soto County, Hydrologic Unit 03100101, on left bank $500 \mathrm{ft}$ upstream from bridge on State Highway $70,1.0$ mi west of post office in Arcadia, 6.1 mi upstream from Joshua Creek, and 36 mi upstream from mouth.

DRAINAGE AREA. $--1,367 \mathrm{mi}^{2}$.

PERIOD OF RECORD ANALYZED.--July 1931 to June 1977.

\begin{tabular}{|c|c|c|c|c|c|c|c|c|}
\hline $\begin{array}{l}\text { Recurrence } \\
\text { interval }\end{array}$ & MAGNITO & $\begin{array}{l}\text { WD FT } \\
\text { owest } \\
\text { for }\end{array}$ & $\begin{array}{l}\text { BQUENCY OF } \\
\text { average } f \\
\text { indicated }\end{array}$ & $\begin{array}{l}\text { ANNUAL } \\
\text { low, in } \\
\text { number }\end{array}$ & $\begin{array}{l}\text { LOW } \\
\text { cub: } \\
\text { of }\end{array}$ & $\begin{array}{l}\text { OWS } \\
\text { feet } \\
\text { secut }\end{array}$ & $\begin{array}{l}\text { ir sec } \\
\text { e day }\end{array}$ & \\
\hline in years & 1 & 7 & 14 & 30 & 60 & 90 & 120 & 183 \\
\hline 2 & 97 & 111 & 120 & 135 & 172 & 222 & 272 & 377 \\
\hline 5 & 63 & 71 & 77 & 88 & 106 & 130 & 150 & 196 \\
\hline 10 & 50 & 57 & 61 & 71 & 84 & 99 & 111 & 140 \\
\hline 20 & 42 & 47 & 51 & 60 & 70 & 80 & 88 & 106 \\
\hline 30 & 38 & 42 & 46 & 55 & 63 & 71 & 78 & 92 \\
\hline 50 & 34 & 38 & 42 & 51 & 57 & 63 & 68 & 78 \\
\hline
\end{tabular}




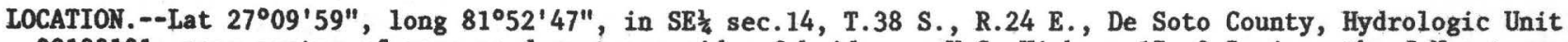
03100101 , near center of span on downstream side of bridge on U.S. Highway $17,0.5$ mi north of Nocatee, and 2.2 mi upstream from mouth.

DRAINAGE AREA. $--132 \mathrm{mi}^{2}$.

PERIOD OF RECORD ANALYZED.--July 1950 to June 1977.

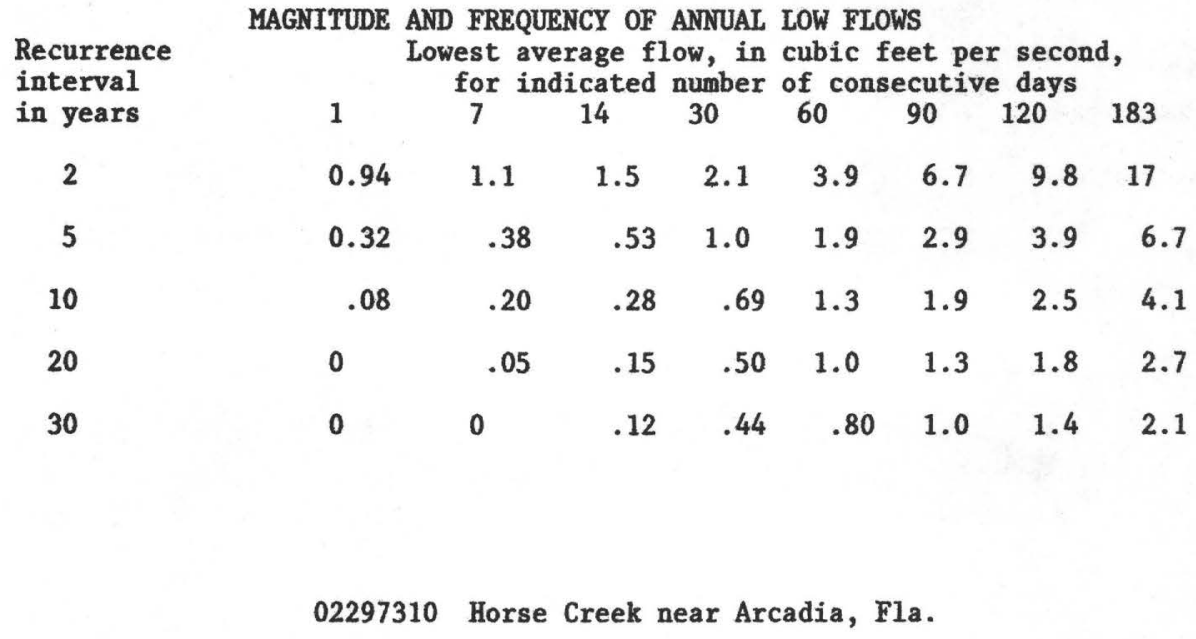

LOCATION.--Lat $27^{\circ} 11^{\prime} 57^{\prime \prime}$, long $81^{\circ} 59^{\prime} 19^{\prime \prime}$, in NW/ sec.2, T.38 S., R.23 E., De Soto County, Hydrologic Unit 03100101 , near center of span on downstream side of bridge on State Highway $72,7.9$ mi west of Arcadia, and 10 mi upstream from mouth.

DRAINAGE AREA. $--218 \mathrm{mi}^{2}$.

PERIOD OF RECORD ANALYZED.--July 1950 to June 1977.

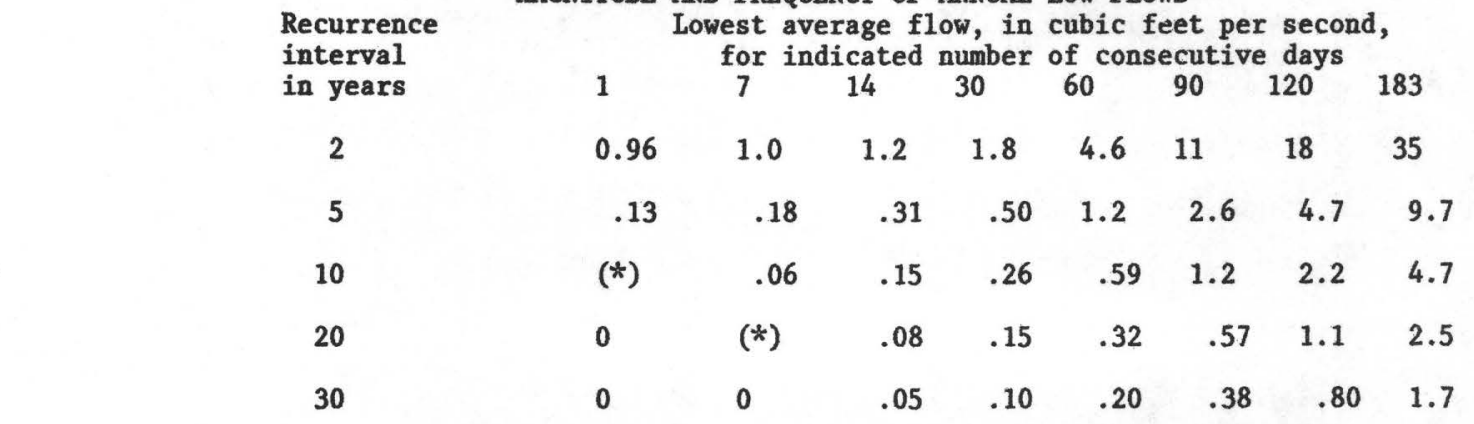

MAGNITUDE AND FREQUENCY OF ANNUAL LOW FLOWS

owest average flow, in cubic feet per second,

$\begin{array}{cccccccc}\text { for indicated number } & \text { of consecutive days } & & \\ 1 & 7 & 14 & 30 & 60 & 90 & 120 & 183\end{array}$

* Less than $0.05 \mathrm{ft}^{3} / \mathrm{s}$. 


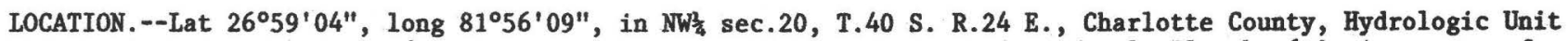
03100101 , near left bank $60 \mathrm{ft}$ upstream from dam, $1.0 \mathrm{mi}$ upstream from Myrtle Slough, $6.0 \mathrm{mi}$ upstream from mouth, and 7.7 mi northeast of Punta Gorda.

DRAINAGE AREA. $--373 \mathrm{mi}^{2}$.

PERIOD OF RECORD ANALYZED.--July 1965 to June 1977.

REMARKS.--Flow regulated by concrete dam. Diversion by city of Punta Gorda for water supply.

Recurrence
interval
in years
2
5
10
20

MAGNITUDE AND FREQUENCY OF ANNUAL LOW FLOWS

Lowest average flow, in cubic feet per second, for indicated number of consecutive days

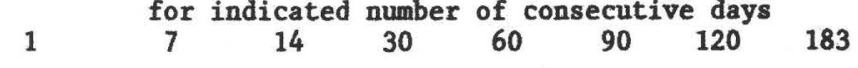

$\begin{array}{llllllll}0 & 0 & 0 & 3.5 & 14 & 27 & 43 & 61\end{array}$

$\begin{array}{llllllll}0 & 0 & 0 & 0 & 2.8 & 10 & 21 & 36\end{array}$

$\begin{array}{llllllll}0 & 0 & 0 & 0 & .65 & 5.0 & 13 & 30\end{array}$

20

0

0

$0 \quad 0$

$.10 \quad 2$.

22 
LOCATION.--Lat $27^{\circ} 14^{\prime} 25^{\prime \prime}$, long $82^{\circ} 18^{\prime} 50^{\prime \prime}$, in SW sec.21, T.37 S., R.20 E., Sarasota County, Hydrologic Unit 03100102 , on right bank 0.5 mi upstream from bridge on State Highway 72 , 1.9 mi upstream from Lower Myakka Lake, $14 \mathrm{mi}$ southeast of Sarasota, and $36 \mathrm{mi}$ upstream from mouth.

DRAINAGE AREA. $--229 \mathrm{mi}^{2}$.

PERIOD OF RECORD ANALYZED.--July 1937 to June 1977.

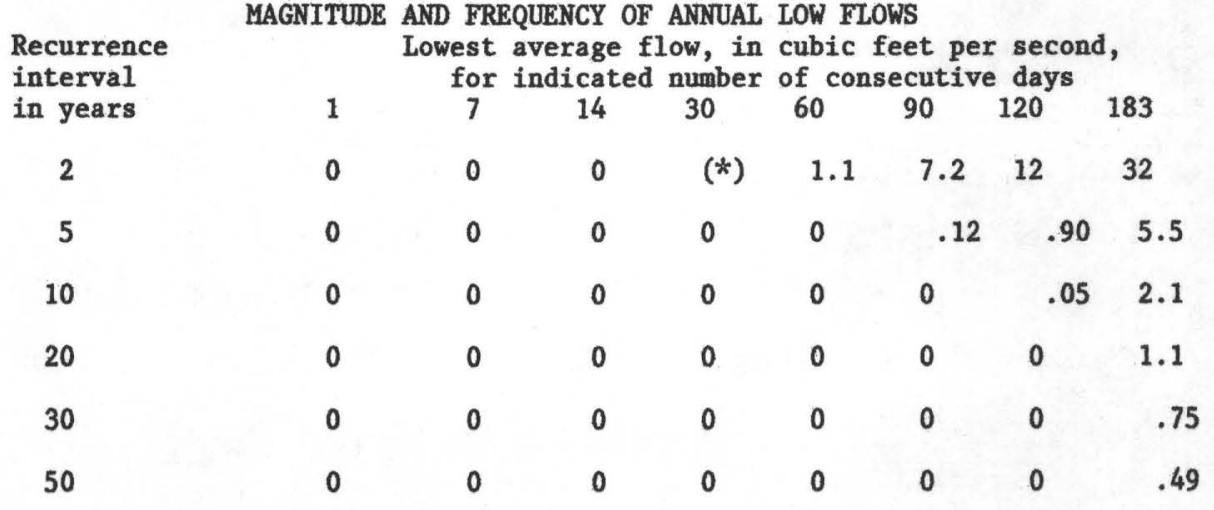

02299470 Big Slough near Murdock, Fla.

LOCATION.--Lat $27^{\circ} 04^{\prime} 15^{\prime \prime}$, long $82^{\circ} 13^{\prime} 05^{\prime \prime}$, in NW sec.21, T.39 S., R.21 R., Sarasota County, Hydrologic Unit 03100102 , near left bank $3 \mathrm{mi}$ upstream from bridge on U.S. Highway $41,5.9$ mi northwest of Murdock, and 7.1 mi upstream from mouth.

DRAINAGE AREA. $--87.5 \mathrm{mi}^{2}$.

PERIOD OF RECORD ANALYZED.--July 1963 to June 1972.

Recurrence
interval
in years
2
5
10

MAGNITUDE AND FREQUENCY OF ANNUAL LOW FLOWS

Lowest average flow, in cubic feet per second,

$\begin{array}{llllllll}\text { for } & \text { fondicated number } & \text { of consecutive days } & & \\ 7 & 7 & 14 & 30 & 60 & 90 & 120 & 183\end{array}$

$\begin{array}{llllllll}0.51 & 0.71 & 1.2 & 1.7 & 2.4 & 4.3 & 8.7 & 13\end{array}$

$\begin{array}{llllllll}.20 & .30 & .44 & .65 & .92 & 1.6 & 2.7 & 3.8\end{array}$

$\begin{array}{llllllll}.12 & .20 & .25 & .38 & .62 & 1.04 & 1.4 & 2.0\end{array}$

* Less than $0.05 \mathrm{ft}^{3} / \mathrm{s}$. 


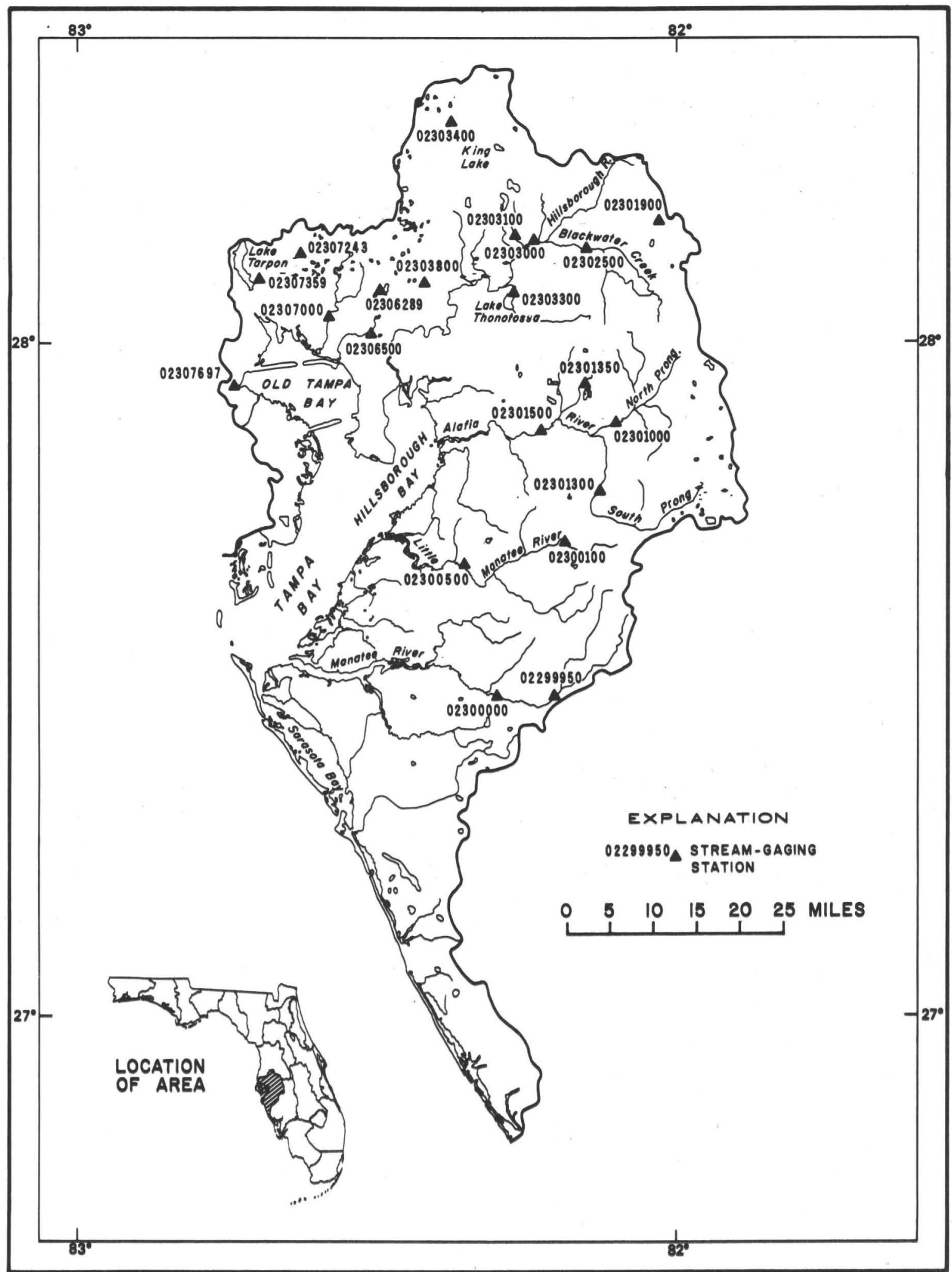

Figure 10.--Location of stream-gaging stations in the Manatee, Little Manatee, Alafia and Hillsborough River basins; Tampa Bay and coastal areas. 
LOCATION.--Lat $27^{\circ} 28^{\prime} 24^{\prime \prime}$, long $82^{\circ} 12^{\prime} 41^{\prime \prime}$, in SE立 sec.33, T.34 S., R.21 E., Manatee County, Hydrologic Unit 03100202 , near center of span on upstream side of bridge on State Highway $64,2.0$ mi downstream from confluence of North and East Forks Manatee River, $6.4 \mathrm{mi}$ east of State Highway 675, $8.4 \mathrm{mi}$ west of Myakka Head, and $36 \mathrm{mi}$ upstream from mouth.

DRAINAGE AREA. $--65.3 \mathrm{mi}^{2}$.

PERIOD OF RECORD ANALYZED.--July 1966 to June 1977.

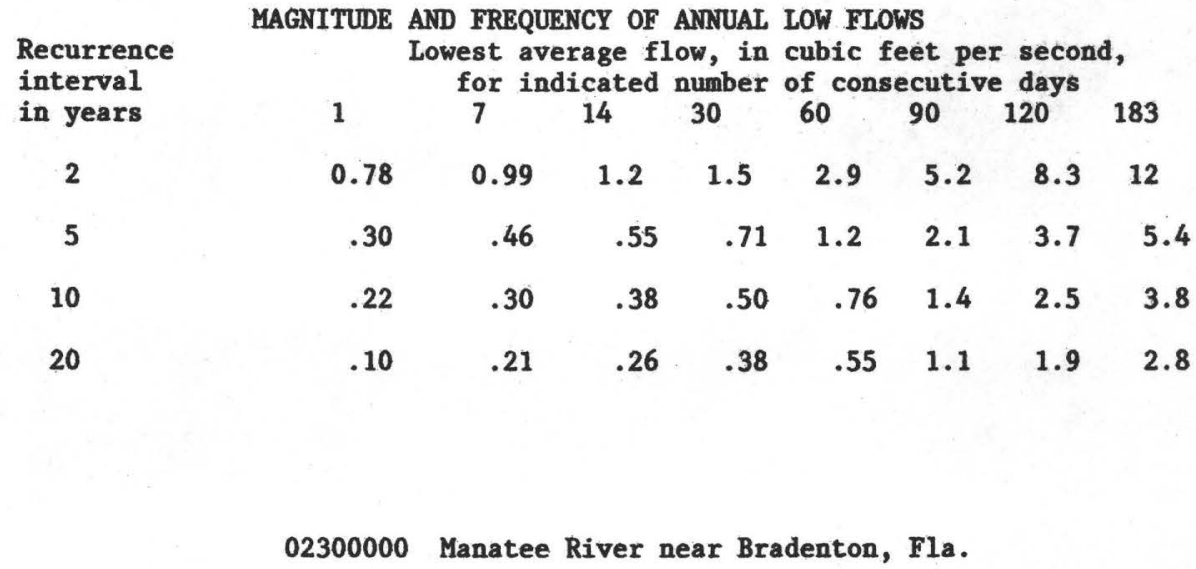

LOCATION.--Lat $27^{\circ} 28^{\prime} 30^{\prime \prime}$, long $82^{\circ} 18^{\prime} 05^{\prime \prime}$, in SWf sec.34, T.34 S., R.20 E., Manatee County, Hydrologic Unit 03100202 , on left bank $150 \mathrm{ft}$ upstream from bridge on State Highway $675,800 \mathrm{ft}$ upstream from Craig Branch, $6 \frac{1}{4} \mathrm{mi}$ northwest of Verna, and $17 \mathrm{mi}$ east of Bradenton.

DRAINAGE ARBA. $--80 \mathrm{mi}^{2}$, approximately.

PERIOD OF RECORD ANALYZED.--July 1939 to June 1965.

Recurrence
interval
in years
2
5
10
20
30

MAGNITUDE AND FREQUENCY OF ANNUAL LOW FLOWS Lowest average flow, in cubic feet per second, for indicated number of consecutive days

interval

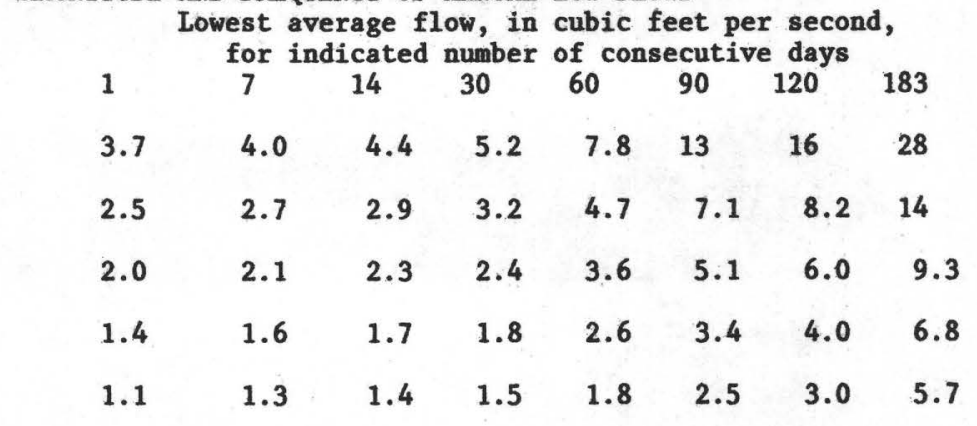




\section{LITTLE MANATEE RIVER BASIN}

02300100 Little Manatee River near Fort Ionesome, Fla.

LOCATION.--Lat $27^{\circ} 42^{\prime} 16^{\prime \prime}$, long $82^{\circ} 11^{\prime} 53^{\prime \prime}$, in NW $\frac{1}{4}$ sec.15, T.32 S., R.21 E., Hillsborough County, Hydrologic Unit 03100203, near right bank on downstream side of bridge on State Highway 674, 0.6 mi upstream from Howard

Prairie Branch, 3.2 mi west of Fort Lonesome, 6.2 mi east of Wimauma, and 30 mi upstream from mouth.

DRAINAGE AREA. $--31.4 \mathrm{mi}^{2}$.

PERIOD OF RECORD ANALYZED.--July 1964 to June 1977.

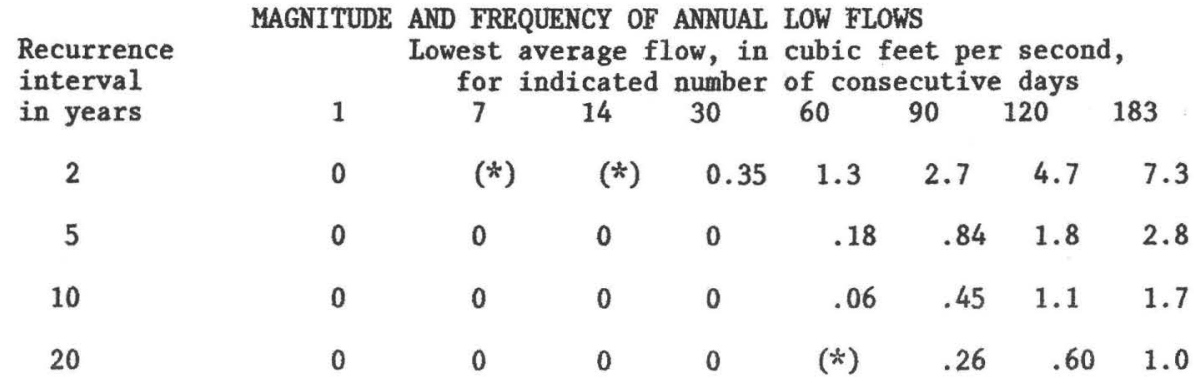

02300500 Little Manatee River near Wimauma, Fla.

LOCATION.--Lat $27^{\circ} 40^{\prime} 15^{\prime \prime}$, long $82^{\circ} 21^{\prime} 10^{\prime \prime}$, in NE $\frac{2}{4}$ sec. 25 , T.32 S., R.19 E., Hillsborough County, Hydrologic Unit 03100203 , near center of span on downstream side of bridge on U.S. Highway $301,1.6$ mi upstream from Cypress Creek, $4.2 \mathrm{mi}$ southwest of Wimauma, and $15 \mathrm{mi}$ upstream from mouth.

DRAINAGE AREA. $--149 \mathrm{mi}^{2}$.

PERIOD OF RECORD ANALYZED.--July 1939 to June 1977.

REMARKS.--Some diversion, $3.3 \mathrm{mi}$ above station by Manatee Power Plant since June 1974.

\begin{tabular}{|c|c|c|c|c|c|c|c|c|}
\hline $\begin{array}{l}\text { Recurrence } \\
\text { interval }\end{array}$ & MAGNITUDE & $\begin{array}{l}D \text { FREQ } \\
\text { west a } \\
\text { for i }\end{array}$ & $\begin{array}{l}\text { NCY OF } \\
\text { rage } f \\
\text { icated }\end{array}$ & $\begin{array}{l}\text { ANNUAL } \\
\text { ow, in } \\
\text { number }\end{array}$ & $\begin{array}{l}\text { LOW F } \\
\text { cubic } \\
\text { of co }\end{array}$ & $\begin{array}{l}\text { WS } \\
\text { eet } p \\
\text { ecuti }\end{array}$ & $\begin{array}{l}\text { r secon } \\
\text { e days }\end{array}$ & \\
\hline in years & 1 & 7 & 14 & 30 & 60 & 90 & 120 & 183 \\
\hline 2 & 7.2 & 8.2 & 9.3 & 12 & 17 & 26 & 35 & 54 \\
\hline 5 & 3.8 & 4.5 & 5.4 & 7.3 & 10 & 15 & 19 & 22 \\
\hline 10 & 2.6 & 3.1 & 3.8 & 5.7 & 8.6 & 11 & 14 & 18 \\
\hline 20 & 1.4 & 1.8 & 2.6 & 4.3 & 6.3 & 8.0 & 10 & 16 \\
\hline 30 & .95 & 1.3 & 2.0 & 3.5 & 5.0 & 6.2 & 8.4 & 15 \\
\hline 50 & .70 & 1.0 & 1.6 & 3.0 & 4.4 & 5.1 & 7.2 & 14 \\
\hline
\end{tabular}

* Less than $0.05 \mathrm{ft}^{3} / \mathrm{s}$. 
LOCATION.--Lat $27^{\circ} 52^{\prime} 59^{\prime \prime}$, long $82^{\circ} 06^{\prime} 03^{\prime \prime}$, in SW sec.10, T.30 S., R.22 E., Hillsborough County, Hydrologic Unit 03100204 , near left bank $300 \mathrm{ft}$ below highway bridge, $0.6 \mathrm{mi}$ north of Keysville, $4.0 \mathrm{mi}$ upstream from confluence with South Prong Alafia River, and $29 \mathrm{mi}$ upstream from mouth of Alafia River at Hillsborough Bay.

DRAINAGE AREA. --135 $\mathrm{mi}^{2}$, approximately.

PERIOD OF RECORD ANALYZED.--July 1950 to June 1977.

Recurrence
interval
in years
2
5
10
20
30

MAGNITUDE AND FREQUENCY OF ANNUAL LOW FLOWS

Lowest average flow, in cubic feet per second, for indicated number of consecutive days

interval

\begin{tabular}{ccccccccc}
1 & \multicolumn{2}{c}{ for } & indicated number of consecutive days & \\
38 & 7 & 14 & 30 & 60 & 90 & 120 & 183 \\
17 & 40 & 43 & 48 & 57 & 68 & 79 & 95 \\
10 & 11 & 23 & 29 & 37 & 47 & 55 & 67 \\
5.4 & 6.2 & 8.6 & 16 & 23 & 33 & 40 & 51 \\
3.6 & 4.0 & 6.3 & 13 & 20 & 30 & 37 & 47
\end{tabular}

02301300 South Prong Alafia River near Lithia, Fla.

\begin{abstract}
LOCATION.--Lat $27^{\circ} 47^{\prime} 47^{\prime \prime}$, long $82^{\circ} 07^{\prime} 04^{\prime \prime}$, in SW sec.9, T.31 S., R.22 E., Hillsborough County, Hydrologic Unit 03100204 , at left bank $12 \mathrm{ft}$ upstream from bridge on county road, 1.5 mi upstream from Halls Branch, $5.0 \mathrm{mi}$ southeast of Lithia, and $7.6 \mathrm{mi}$ upstream from mouth.
\end{abstract}

DRAINAGE AREA. $--107 \mathrm{mi}^{2}$.

PERIOD OF RECORD ANALYZED.--July 1963 to June 1977.

Recurrence
interval
in years
2
5
10
20

MAGNITUDE AND FREQUENCY OF ANNUAL LOW FLOWS

Lowest average flow, in cubic feet per second, for indicated number of consecutive days

\begin{tabular}{lcccccccc}
\multicolumn{7}{c}{ for } & indicated number of consecutive days & \multicolumn{2}{c}{ of } \\
1 & 7 & 14 & 30 & 60 & 90 & 120 & 183 \\
11 & 14 & 17 & 20 & 31 & 40 & 47 & 59 \\
3.7 & 4.8 & 5.2 & 9.0 & 14 & 19 & 23 & 32 \\
2.1 & 2.9 & 3.3 & 6.6 & 10 & 13 & 16 & 25 \\
1.3 & 1.9 & 2.2 & 5.0 & 7.0 & 9.0 & 12 & 19
\end{tabular}


LOCATION.--Lat $27^{\circ} 56^{\prime} 24^{\prime \prime}$, long $82^{\circ} 09^{\prime} 13^{\prime \prime}$, in SE $\frac{1}{4}$ sec.24, T.29 S., R.21 E., Hillsborough County, Hydrologic Unit 03100204 , near left bank, 1,200 ft above culvert on State Highway 60, 2.4 mi upstream from Pleasant Grove Reservoir control structure, and $3.0 \mathrm{mi}$ west of Hopewell.

DRAINAGE AREA. $--8.65 \mathrm{mi}^{2}$.

PERIOD OF RECORD ANALYZED.--July 1967 to June 1977.

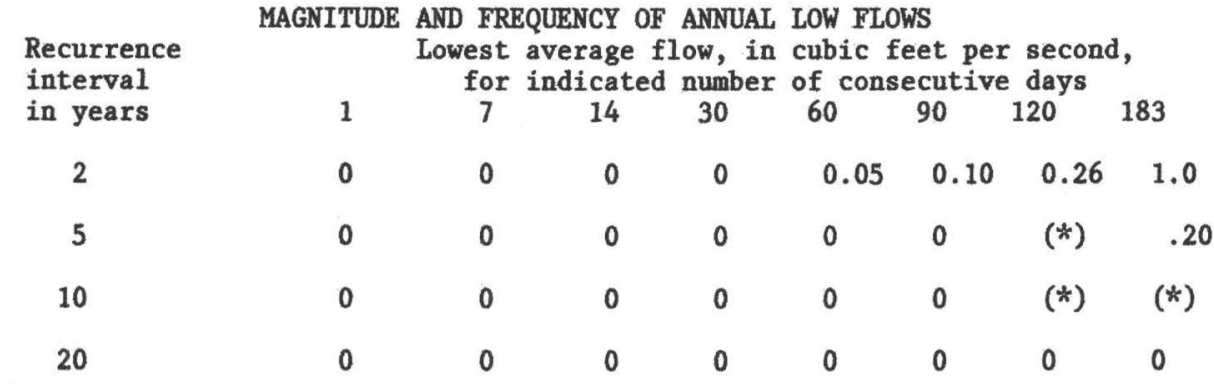

02301500 Alafia River at Lithia, Fla.

LOCATION.--Lat $27^{\circ} 52^{\prime} 19^{\prime \prime}$, long $82^{\circ} 12^{\prime} 41^{\prime \prime}$, in NE条 sec.16, T.30 S., R.21 E., Hillsborough County, Hydrologic Unit 03100204 , near center of span on downstream side of bridge on State Highway $640,2.0$ mi upstream from Little Fishhawk Creek, $4.3 \mathrm{mi}$ west of Lithia, and $16 \mathrm{mi}$ upstream from mouth.

DRAINAGE AREA. $--335 \mathrm{mi}^{2}$, approximately.

PERIOD OF RECORD ANALYZED.--July 1933 to June 1977.

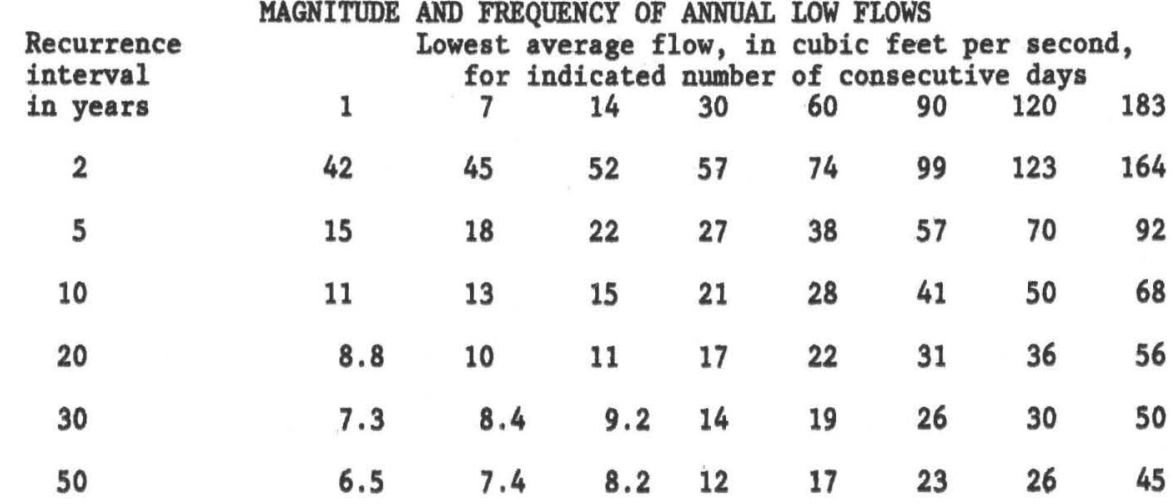

* Less than $0.05 \mathrm{ft}^{3} / \mathrm{s}$. 


\section{HILLSBOROUGH RIVER BASIN}

02301900 Fox Branch near Socrum, Fla.

LOCATION.--Lat $28^{\circ} 10^{\prime} 55^{\prime \prime}$, long $82^{\circ} 00^{\prime} 45^{\prime \prime}$, in NE $\frac{3}{4}$ sec.33, T.26 S., R.23 E., Polk County, Hydrologic Unit 03100205, near center of span on upstream side of bridge on Rock Ridge Road, 1.1 mi northeast of Socrum, 8.7 mi upstream from mouth, and $10 \mathrm{mi}$ north of Lakeland.

DRAINAGE AREA. $--9.5 \mathrm{mi}^{2}$, approximately.

PERIOD OF RECORD ANALYZED.--July 1964 to June 1977.

REMARKS.--Some diversion at times by pumpage for irrigation.

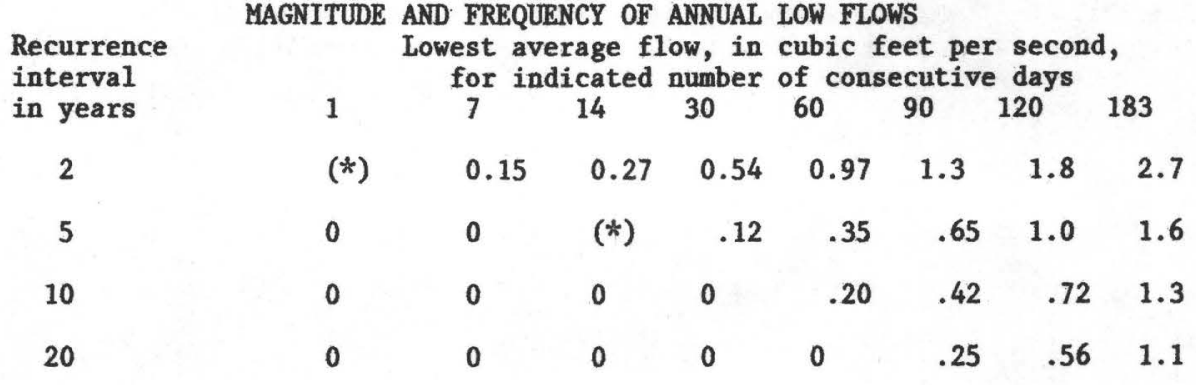

02302500 Blackwater Creek near Knights, Fla.

LOCATION.--Lat $28^{\circ} 08^{\prime} 25^{\prime \prime}$, long $82^{\circ} 09^{\prime} 00^{\prime \prime}$, in NW sec.18, T.27 S., R.22 E., Hillsborough County, Hydrologic Unit 03100205 , on downstream side of center pier of bridge on State Highway $39,2.0$ mi downstream from Itchepackesassa Creek, $4.4 \mathrm{mi}$ northwest of Knights, and $5.2 \mathrm{mi}$ upstream from mouth.

DRAINAGE AREA. $--110 \mathrm{mi}^{2}$, approximately.

PERIOD OF RECORD ANALYZED.--July 1951 to June 1978.

REMARKS.--Several diversions above station for irrigation.

\begin{tabular}{|c|c|c|c|c|c|c|c|c|}
\hline $\begin{array}{l}\text { Recurrence } \\
\text { interval }\end{array}$ & MAGNITUDE & $\begin{array}{l}\text { FREQ } \\
\text { est a } \\
\text { for ir }\end{array}$ & $\begin{array}{l}C Y \text { OF } \\
\text { age } f 1 \\
\text { cated }\end{array}$ & $\begin{array}{l}\text { ANNUAL } \\
\text { ow, in } \\
\text { number }\end{array}$ & $\begin{array}{l}\text { LOW FI } \\
\text { cubic } \\
\text { of con }\end{array}$ & $\begin{array}{l}\text { WS } \\
\text { eet pe } \\
\text { ecutiv }\end{array}$ & $\begin{array}{l}r \text { secon } \\
\text { e days }\end{array}$ & \\
\hline in years & 1 & 7 & 14 & 30 & 60 & 90 & 120 & 183 \\
\hline 2 & 3.3 & 5.2 & 7.1 & 8.0 & 11 & 15 & 19 & 29 \\
\hline 5 & .92 & 2.1 & 2.9 & 5.5 & 7.6 & 9.6 & 12 & 17 \\
\hline 10 & .62 & .94 & 1.4 & 4.5 & 6.0 & 7.6 & 9.9 & 13 \\
\hline 20 & .42 & .60 & .71 & 2.3 & 3.7 & 6.0 & 8.7 & 11 \\
\hline 30 & .20 & .35 & .48 & 1.8 & 2.3 & 4.3 & 8.1 & 10 \\
\hline
\end{tabular}

* Less than $0.05 \mathrm{ft}^{3} / \mathrm{s}$. 
LOCATION.--Lat $28^{\circ} 08^{\prime} 59^{\prime \prime}$, long $82^{\circ} 13^{\prime} 57^{\prime \prime}$, in SW sec.8, T.27 S., R.21 E., Hillsborough County, Hydrologic Unit 03100205, on left bank $30 \mathrm{ft}$ downstream from footbridge in Hillsborough River State Park, $1.2 \mathrm{mi}$ downstream from Blackwater Creek, $6.5 \mathrm{mi}$ southwest of Zephyrhills, and $40 \mathrm{mi}$ upstream from mouth.

DRAINAGE AREA. $--220 \mathrm{mi}^{2}$, approximately.

PERIOD OF RECORD ANALYZED.--July 1940 to June 1977.

\begin{tabular}{|c|c|c|c|c|c|c|c|c|}
\hline \multirow{2}{*}{$\begin{array}{l}\text { Recurrence } \\
\text { interval } \\
\text { in years }\end{array}$} & MAGNITUD & $\begin{array}{l}D \mathrm{FI} \\
\text { wes } \\
\text { fol }\end{array}$ & $\begin{array}{l}\text { JCY } \\
\text { cage } \\
\text { icat }\end{array}$ & $\begin{array}{l}\text { ANN } \\
\text { ow, } \\
\text { numl }\end{array}$ & $\begin{array}{l}\text { LOW } \\
\text { cubi } \\
\text { of }\end{array}$ & $\begin{array}{l}\text { WS } \\
\text { eet } \\
\text { ecu }\end{array}$ & $\begin{array}{l}\text { sec } \\
\text { day }\end{array}$ & \\
\hline & 1 & 7 & 14 & 30 & 60 & 90 & $120^{\circ}$ & 183 \\
\hline 2 & 68 & 70 & 72 & 75 & 81 & 88 & 97 & 120 \\
\hline 5 & 57 & 59 & 60 & 62 & 66 & 70 & 74 & 84 \\
\hline 10 & 52 & 53 & 55 & 57 & 59 & 63 & 65 & 72 \\
\hline 20 & 48 & 49 & 51 & 52 & 55 & 58 & 60 & 65 \\
\hline 30 & 46 & 47 & 48 & 50 & 52 & 55 & 57 & 61 \\
\hline 50 & 44 & 45 & 46 & 48 & 50 & 53 & 55 & 58 \\
\hline
\end{tabular}

02303100 New River near Zephyrhills, Fla.

LOCATION.--Lat $28^{\circ} 09^{\prime} 55^{\prime \prime}$, long $82^{\circ} 15^{\prime} 55^{\prime \prime}$, in NWz sec.1, T.27 S., R.20 E., Hillsborough County, Hydrologic Unit 03100205 , near left bank $100 \mathrm{ft}$ upstream from bridge on State Highway $579,1.4$ mi upstream from small tributary, $1.8 \mathrm{mi}$ upstream from mouth, and $7 \mathrm{mi}$ southwest of Zephyrhills.

DRAINAGE AREA. $--15 \mathrm{mi}^{2}$, approximately.

PERIOD OF RECORD ANALYZED.--July 1964 to June 1974.

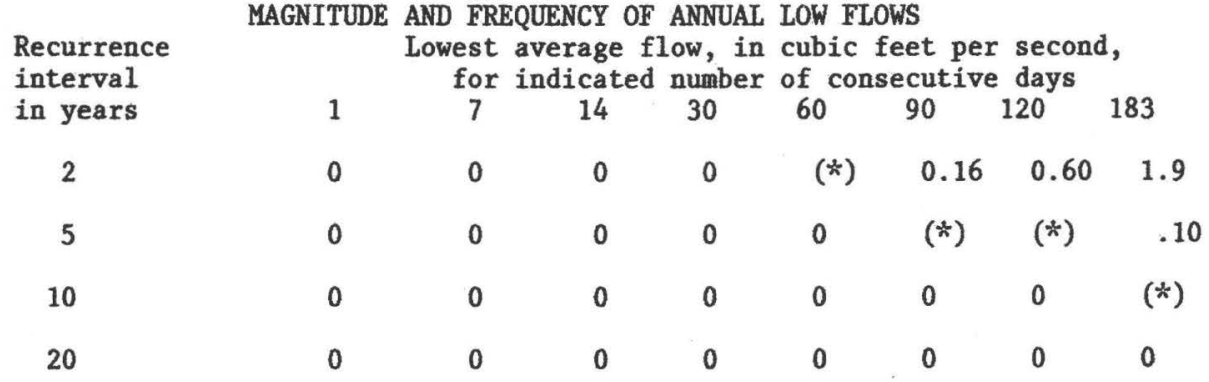

* Less than $0.05 \mathrm{ft}^{3} / \mathrm{s}$. 
LOCATION.--Lat $28^{\circ} 04^{\prime} 04^{\prime \prime}$, long $82^{\circ} 16^{\prime} 04^{\prime \prime}$, in NWt sec.12, T.28 S., R.20 E., Hillsborough County, Hydrologic Unit 03100205, on right bank $40 \mathrm{ft}$ upstream from control, $50 \mathrm{ft}$ upstream from bridge, $500 \mathrm{ft}$ downstream from Lake Thonotosassa, $2.0 \mathrm{mi}$ northeast of Thonotosassa, and $2.8 \mathrm{mi}$ upstream from mouth.

DRAINAGE AREA. - -60 mi ${ }^{2}$, approximately.

PERIOD OF RECORD ANALYZED.--July 1957 to June 1958, July 1971 to June 1977.

REMARKS.--Flow regulated by manipulation of stoplogs and vertical lift gates in control. Prior to December 1975, flow regulated by manipulation of stoplogs in control.

$\begin{aligned} & \text { Recurrence } \\ & \text { interval } \\ & \text { in years }\end{aligned}$
2
5
10

MAGNITUDE AND FREQUENCY OF ANDUAL LOW FLOWS

Lowest average flow, in cubic feet per second, for indicated number of consecutive days

\section{interval}

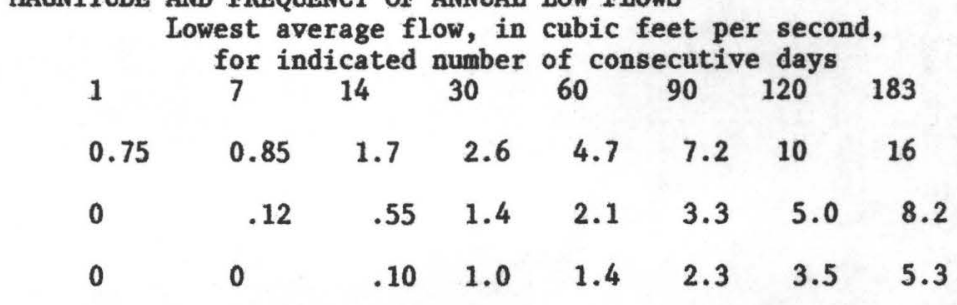

02303400 Cypress Creek near San Antonio, Fla.

LOCATION.--Lat $28^{\circ} 19^{\prime} 2^{\prime \prime}$, long $82^{\circ} 23^{\prime} 03^{\prime \prime}$, in SW sec.11, T.25 S., R. 19 E., Pasco County, Hydrologic Unit 03100205, at center on downstream side of box culverts on State Highway 52, 3.3. mi downstream from Bee Treee Branch, $6.8 \mathrm{mi}$ west of San Antonio, $12 \mathrm{mi}$ west of Dade City, and $25 \mathrm{mi}$ upstream from mouth.

DRAINAGE AREA. $--56.0 \mathrm{mi}^{2}$.

PERIOD OF RECORD ANALYZED.--July 1963 to June 1978.

Recurrence
interval
in years
2
5
10
20

MAGNITUDE AND FREQUENCY OF ANNUAL LOW FLOWS Lowest average flow, in cubic feet per second,

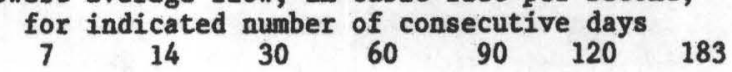

$\begin{array}{llllllll}1 & 7 & 14 & 30 & 60 & 90 & 120 & 183\end{array}$

\footnotetext{
* Less than $0.05 \mathrm{ft}^{3} / \mathrm{s}$.
} 
LOCATION.--Lat $28^{\circ} 05^{\prime} 20^{\prime \prime}$, long $82^{\circ} 24^{\prime} 33^{\prime \prime}$, in SE⿺ sec.33, T.27 S., R.19 E., Hillsborough County, Hydrologic Unit 03100205 , near center of span on downstream side of bridge on State Highway 581, 1.2 mi downstream from Thirteenmile Run, $2.5 \mathrm{mi}$ upstream from mouth, and 5.0 mi northeast of Sulphur Springs.

DRAINAGE AREA.--160 mi ${ }^{2}$, approximately.

PERIOD OF RECORD ANALYZED.--July 1964 to June 1977.

Recurrence
interval
in years
2
5
10
20

MAGNITUDE AND FREQUENCY OF ANNUAL LOW FLOWS

Lowest average flow, in cubic feet per second, for indicated number of consecutive days

$\begin{array}{lllllll}7 & 14 & 30 & 60 & 90 & 120 & 183\end{array}$

$\begin{array}{llllllll}1 & 0 & 0 & 0 & 0.20 & 3.2 & 14 & 27\end{array}$

$\begin{array}{llllllll}0 & 0 & 0 & 0 & (*) & .40 & 6.4 & 14\end{array}$

$\begin{array}{llllllll}0 & 0 & 0 & 0 & 0 & .14 & 4.6 & 10\end{array}$

* Less than $0.05 \mathrm{ft}^{3} / \mathrm{s}$. 
LOCATION.--Lat $28^{\circ} 02^{\prime} 35^{\prime \prime}$, long $82^{\circ} 30^{\prime} 42^{\prime \prime}$, in SW $\frac{3}{4}$ sec.16, T.28 S., R.18 E., Hillsborough County, Hydrologic Unit 03100206, $25 \mathrm{ft}$ upstream from culverts on private road, $160 \mathrm{ft}$ upstream from Gunn Highway, 1.7 mi downstream from Lake Ellen, $3.5 \mathrm{mi}$ west of intersection of Interstate 75 and Busch Boulevard at Sulphur Springs.

DRAINAGE AREA. $--7.43 \mathrm{mi}^{2}$.

PERIOD OF RECORD ANALYZED.--July 1952 to June 1970, July 1972 to June 1977.

REMARKS.--Flow affected by regulation of control structures above station. Since February 10, 1953, flow occasionally diverted from Hillsborough River basin into the upper portion of Sweetwater Creek basin.

Since January 1970, flow has been diverted from basin (below station) through Channel G to Rocky Creek.

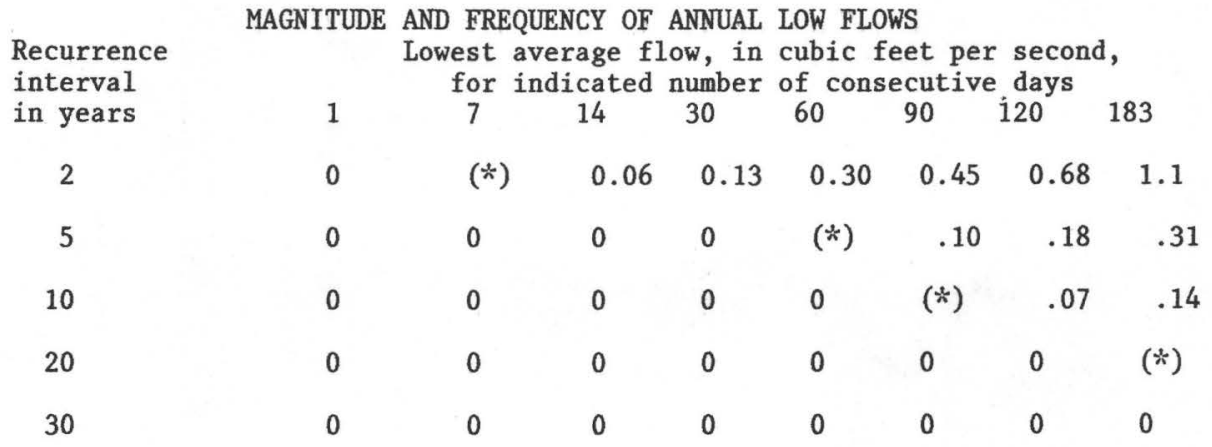

02307000 Rocky Creek near Sulphur Springs, Fla.

LOCATION.--Lat $28^{\circ} 02^{\prime} 12^{\prime \prime}$, long $82^{\circ} 34^{\prime} 34^{\prime \prime}$, in NWh sec.23, T.28 S., R.17 E., Hillsborough County, Hydrologic Unit 03100206 , on left bank 75 ft upstream from concrete control, 2.8 mi downstream from Brushy Creek, $5.8 \mathrm{mi}$ upstream from mouth, and $7.4 \mathrm{mi}$ west of intersection of Interstate 75 and Busch Boulevard at Sulphur Springs.

DRAINAGE AREA.--35 $\mathrm{mi}^{2}$, approximately.

PERIOD OF RECORD ANALYZED.--July 1953 to June 1977.

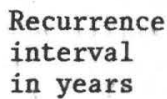

2

5

10

20

30

MAGNITUDE AND FREQUENCY OF ANNUAL LOW FLOWS

Lowest average flow, in cubic feet per second, for indicated number of consecutive days

$\begin{array}{llllllll}1 & 7 & 14 & 30 & 60 & 90 & 120 & 183\end{array}$

5

10

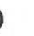

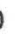

(2)
1.1

.42

.20

(*)

0
1.4

.54

.28

.05

0
1.6

.64

.35

.08

(*)
2.0

2.7

$1.1 \quad 1.6$

.54

.12

$(*)$

.85

1.0
8.7

$\begin{array}{lll}2.2 & 3.0 & 3.7\end{array}$

$\begin{array}{ll}1.3 & 1.7\end{array}$

$2.2 \quad 3.2$

1.3

1.7

2.8

2.5

* Less than $0.05 \mathrm{ft}^{3} / \mathrm{s}$. 
LOCATION.--Lat $28^{\circ} 08^{\prime} 05^{\prime \prime}$, long $82^{\circ} 35^{\prime} 40^{\prime \prime}$, in sec.10, T.27 S., R.17 E., Hillsborough County, Hydrologic Unit 03100206, on left bank $20 \mathrm{ft}$ upstream from bridge on State Highway $232,30 \mathrm{ft}$ downstream from outlet of Keystone Lake, and $3.2 \mathrm{mi}$ south of Odessa.

DRAINAGE AREA. $--10 \mathrm{mi}^{2}$, approximately.

PERIOD OF RECORD ANALYZED.--July 1946 to June 1956.

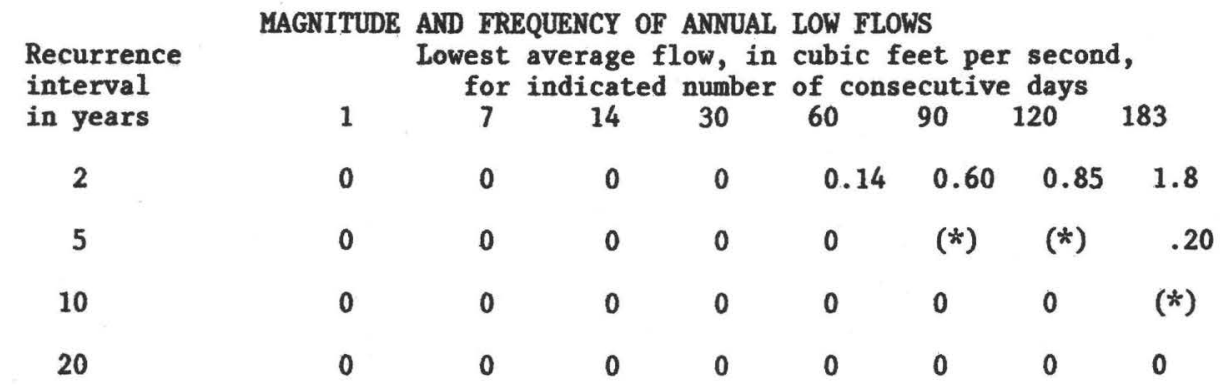

02307359 Brooker Creek near Tarpon Springs, Fla.

LOCATION.--Iat $28^{\circ} 05^{\prime} 4^{\prime \prime}$, long $82^{\circ} 41^{\prime} 15^{\prime \prime}$, in NE $\frac{1}{4}$ sec.34, T.27 S., R.16 E., Pinellas County, Hydrologic Unit 03100206, on right bank, 1.9 mi upstream from mouth, and 5 mi southeast of Tarpon Springs.

DRAINAGE AREA. $--30 \mathrm{mi}^{2}$, approximately.

PERIOD OF RECORD ANALYZED.--July 1951 to June 1977.

\begin{tabular}{cccccccrrr}
$\begin{array}{l}\text { Recurrence } \\
\text { interval } \\
\text { in years }\end{array}$ & 1 & \multicolumn{7}{c}{$\begin{array}{c}\text { Lowest average flow, in cubic feet per second, } \\
\text { for indicated number of consecutive days }\end{array}$} \\
2 & 0 & 7 & 14 & 30 & 60 & 90 & 120 & 183 \\
5 & 0 & 0 & 0 & $(*)$ & 0.30 & 1.6 & 4.4 \\
10 & 0 & 0 & 0 & 0 & 0 & $(*)$ & .23 & .90 \\
20 & 0 & 0 & 0 & 0 & 0 & 0 & .10 & .37 \\
30 & 0 & 0 & 0 & 0 & 0 & 0 & $(*)$ & .14 \\
& 0 & 0 & 0 & 0 & 0 & 0 & $(*)$ & $(*)$
\end{tabular}

MAGNITUDE AND FREQUENCY OF ANNUAL LOW FLOWS

* Less than $0.05 \mathrm{ft}^{3} / \mathrm{s}$. 
TAMPA BAY AND COASTAL AREAS

02307697 Alligator Creek at Safety Harbor, Fla.

LOCATION.--Lat $27^{\circ} 58^{\prime} 45^{\prime \prime}$, long $82^{\circ} 41^{\prime} 43^{\prime \prime}$, in SW sec.10, T.29 S., R.16 E., Pinellas County, Hydrologic Unit 03100206 , on right upstream wingwall of concrete control structure, 30 ft upstream from bridge on Bayshore Drive and $0.8 \mathrm{mi}$ southwest of Safety Harbor.

DRAINAGE AREA.--9.0 $\mathrm{mi}^{2}$, approximately.

PERIOD OF RECORD ANALYZED.--July 1950 to June 1974.

REMARKS.--Some diversions may occur above station for irrigation.

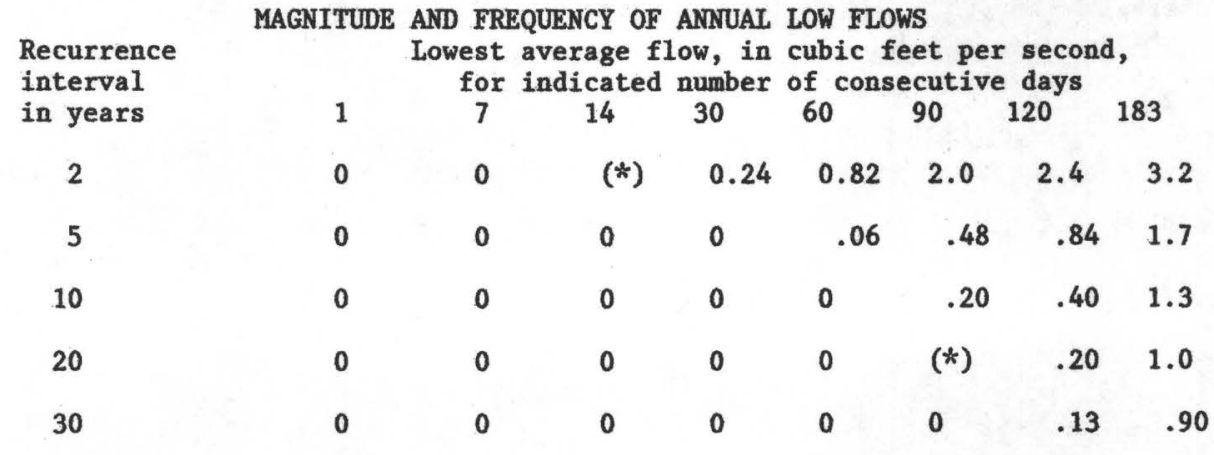

* Less than $0.05 \mathrm{ft}^{3} / \mathrm{s}$. 


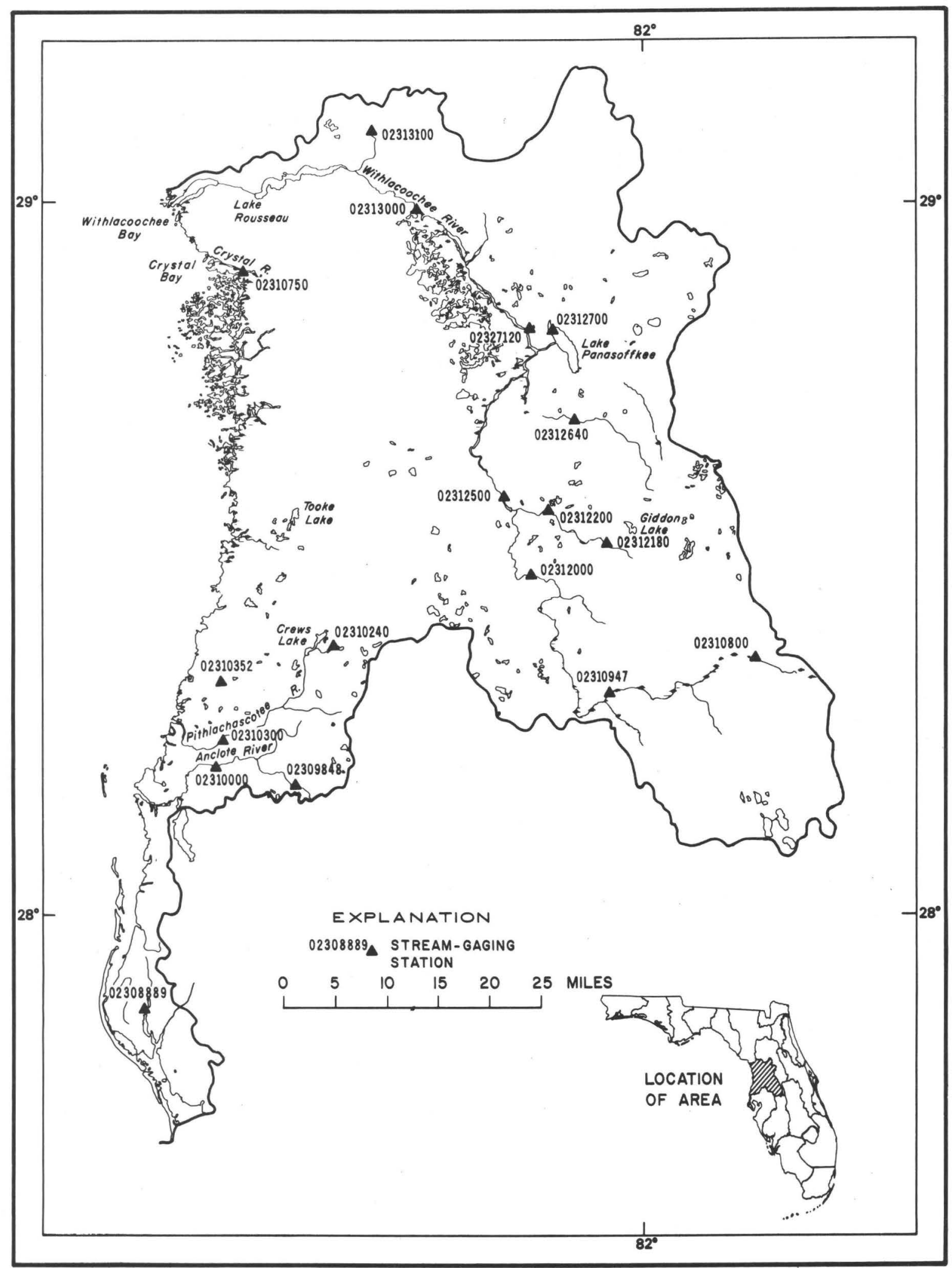

Figure 11.--Location of stream-gaging stations in the coastal area from Tampa Bay to Withlacoochee River and the Withlacoochee River basin. 
LOCATION.--Lat $27^{\circ} 50^{\prime} 20^{\prime \prime}$, long $82^{\circ} 46^{\prime} 50^{\prime \prime}$, in sec.27, T.30 S., R. 15 E., Pinellas County, Hydrologic Unit 03100207 , on south shore of Seminole Lake, $250 \mathrm{ft}$ west of highway bridge across spillway channel, and $5.2 \mathrm{mi}$ south of Largo.

DRAINAGE AREA.--14 $\mathrm{mi}^{2}$, approximately.

PERIOD OF RECORD ANALYZED.--July 1951 to June 1971.

REMARKS.--Greater part of inflow to Seminole Lake is regulated by pumps at north dam 3.0 mi above station. Pumpage at north dam represents natural flow of tributary above dam.

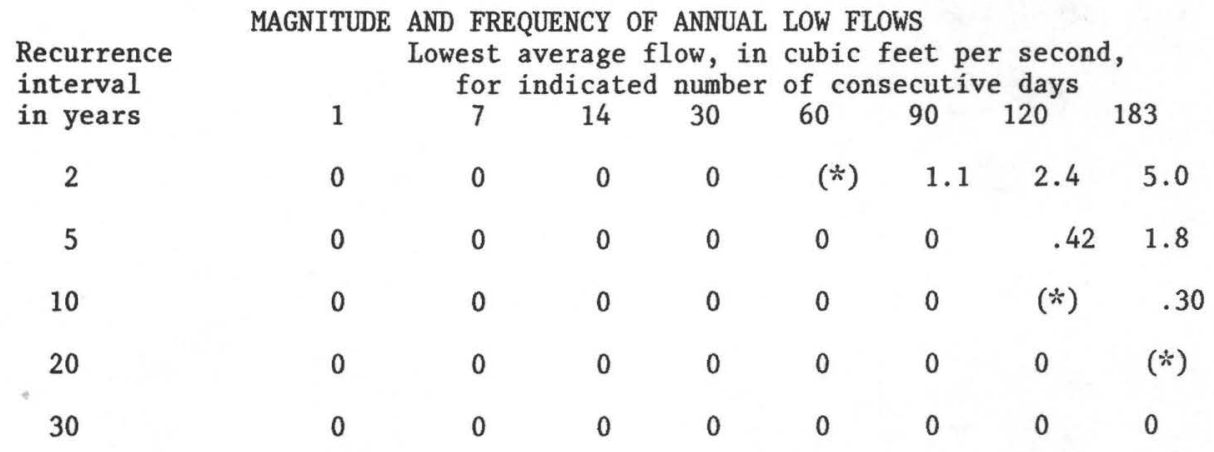

02309848 South Branch Anclote River near Odessa, Fla.

LOCATION.--Lat $28^{\circ} 11^{\prime} 08^{\prime \prime}$, long $82^{\circ} 33^{\prime} 13^{\prime \prime}$, in SE $\frac{1}{4}$ sec.25, T.26 S., R.17 E., Pasco County, Hydrologic Unit 03100207, near left bank $30 \mathrm{ft}$ downstream from bridge on State Highway 54, $2.5 \mathrm{mi}$ east of 0 dessa, $3.0 \mathrm{mi}$ upstream from unnamed tributary, and $5.2 \mathrm{mi}$ upstream from mouth.

DRAINAGE AREA. $--17.1 \mathrm{mi}^{2}$.

PERIOD OF RECORD ANALYZED.--July 1970 to June 1978.

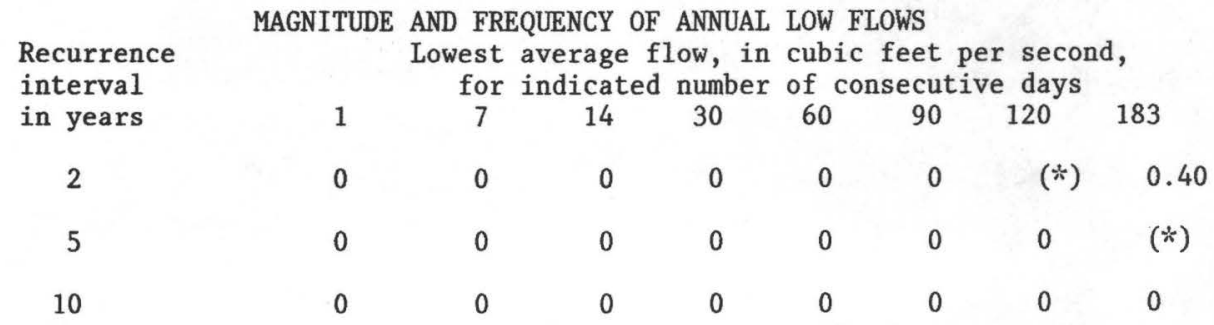

* Less than $0.05 \mathrm{ft}^{3} / \mathrm{s}$ : 


\section{COASTAL AREA FROM TAMPA BAY TO WITHLACOOCHEE RIVER}

02310000 Anclote River near Elfers, Fla.

LOCATION.--Lat $28^{\circ} 12^{\prime} 50^{\prime \prime}$, long $82^{\circ} 40^{\prime} 00^{\prime \prime}$, in NE $\frac{3}{4}$ sec.23, T.26 S., R.16 E., Pasco County, Hydrologic Unit 03100207 , on left bank $40 \mathrm{ft}$ downstream from bridge on State Highway 54, 3.5 mi east of Elfers, and 16 mi upstream from mouth.

DRAINAGE AREA. $--72.5 \mathrm{mi}^{2}$.

PERIOD OF RECORD ANALYZED.--July 1946 to June 1977.

\begin{tabular}{|c|c|c|c|c|c|c|c|c|}
\hline $\begin{array}{l}\text { Recurrence } \\
\text { interval }\end{array}$ & MAGNITUDE & $\begin{array}{l}\text { FREQL } \\
\text { west a } \\
\text { for in }\end{array}$ & $\begin{array}{l}\text { ICY OF } \\
\text { rage f } \\
\text { cated }\end{array}$ & $\begin{array}{l}\text { ANNUAL } \\
\text { ow, in } \\
\text { number }\end{array}$ & $\begin{array}{l}\text { LOW F } \\
\text { cubic } \\
\text { of }\end{array}$ & $\begin{array}{l}\text { WS } \\
\text { eet p } \\
\text { ecuti }\end{array}$ & $\begin{array}{l}\text { secor } \\
\text { e days }\end{array}$ & \\
\hline in years & 1 & 7 & 14 & 30 & 60 & 90 & $120^{\circ}$ & 183 \\
\hline 2 & 2.4 & 2.6 & 2.8 & 3.1 & 3.5 & 4.5 & 9.4 & 16 \\
\hline 5 & 1.6 & 2.0 & 2.2 & 2.4 & 2.9 & 3.4 & 4.0 & 8.6 \\
\hline 10 & 1.3 & 1.6 & 1.8 & 2.1 & 2.7 & 3.1 & 3.6 & 6.0 \\
\hline 20 & 1.0 & 1.2 & 1.3 & 1.8 & 2.5 & 2.9 & 3.3 & 4.3 \\
\hline 30 & .90 & 1.0 & 1.1 & 1.6 & 2.4 & 2.8 & 3.1 & 3.6 \\
\hline 50 & .80 & .90 & 1.0 & 1.5 & 2.3 & 2.6 & 3.0 & 3.2 \\
\hline
\end{tabular}

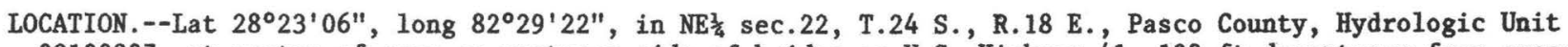
03100207 , at center of span on upstream side of bridge on U.S. Highway 41, 100 ft downstream from concrete control structure at outlet of Pasco Lake, $0.3 \mathrm{mi}$ north of Loyce, $2.7 \mathrm{mi}$ upstream from mouth, and $4.4 \mathrm{mi}$ southwest of Masaryktown.

DRAINAGE AREA. $--43 \mathrm{mi}^{2}$, approximately.

PERIOD OF RECORD ANALYZED.--July 1964 to June 1977.

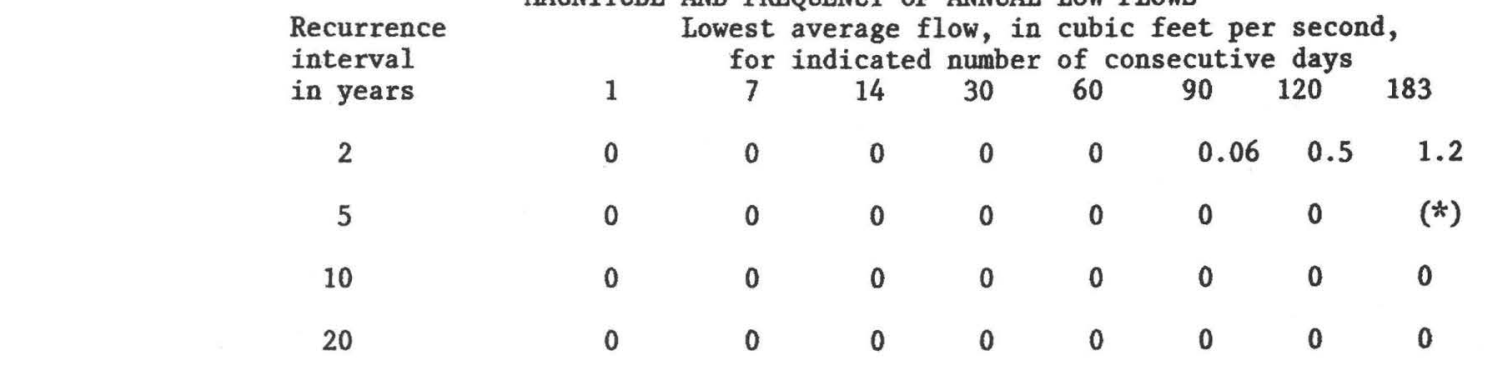

MAGNITUDE AND FREQUENCY OF ANNUAL LOW FLOWS

$*$ Less than $0.05 \mathrm{ft}^{3} / \mathrm{s}$. 
LOCATION.--Lat $28^{\circ} 15^{\prime} 19^{\prime \prime}$, long $82^{\circ} 39^{\prime} 37^{\prime \prime}$, in NW/ sec.1, T.26 S., R.16 E., Pasco County, Hydrologic Unit 03100207 , on left bank downstream from end of private road, $3.8 \mathrm{mi}$ east of New Port Richey, and $9.4 \mathrm{mi}$ upstream from mouth.

DRAINAGE AREA. - - $182 \mathrm{mi}^{2}$.

PERIOD OF RECORD ANALYZED.--July 1963 to June 1977.

\begin{tabular}{|c|c|c|c|c|c|c|c|c|}
\hline \multirow{2}{*}{$\begin{array}{l}\text { Recurrence } \\
\text { interval } \\
\text { in years }\end{array}$} & MAGNITUDE & $\begin{array}{l}\text { FREQU } \\
\text { west av } \\
\text { for in }\end{array}$ & $\begin{array}{l}\text { ICY OF } \\
\text { cage fl } \\
\text { cated }\end{array}$ & $\begin{array}{l}\text { ANNUAL } \\
\text { OW, in } \\
\text { number }\end{array}$ & $\begin{array}{l}\text { LOW FLC } \\
\text { cubic } \\
\text { of cons }\end{array}$ & $\begin{array}{l}\text { WS } \\
\text { eet pe: } \\
\text { ecutiv }\end{array}$ & $\begin{array}{l}\text { secon } \\
\text { days }\end{array}$ & \\
\hline & 1 & 7 & 14 & 30 & 60 & 90 & 120 & 183 \\
\hline 2 & 0.77 & 0.82 & 0.86 & 1.0 & 1.7 & 3.4 & 6.5 & 11 \\
\hline 5 & .55 & .58 & .65 & .77 & 1.0 & 1.6 & 3.2 & 5.6 \\
\hline 10 & .46 & .49 & .58 & .72 & .77 & 1.1 & 2.2 & 4.2 \\
\hline 20 & .38 & .42 & .54 & .70 & .65 & .83 & 1.7 & 3.3 \\
\hline
\end{tabular}

02310352 Bear Creek at Plaza Drive near Hudson, Fla.

LOCATION.--Lat $28^{\circ} 19^{\prime} 38^{\prime \prime}$, long $82^{\circ} 39^{\prime} 59^{\prime \prime}$, in NW/ sec.12, T.25 S., R.16 E., Pasco County, Hydrologic Unit 03100207 , on right bank $45 \mathrm{ft}$ upstream from culverts on Plaza Drive, 0.3 mi upstream from Bear Sink, and $3.0 \mathrm{mi}$ southeast of Hudson.

DRAINAGE AREA. $--29.2 \mathrm{mi}^{2}$,

PERIOD OF RECORD ANALYZED.--July 1970 to June 1977.

Recurrence
interval
in years
2
5
10

MAGNITUDE AND FREQUENCY OF ANNUAL LOW FLOWS

Lowest average flow, in cubic feet per second, $\begin{array}{cccccc}\text { for indicated number of consecutive days } & \\ 7 & 14 & 30 & 60 & 90 & 120\end{array}$

$\begin{array}{rrrrrrrrrr}2 & 0 & 0.05 & 0.10 & 0.48 & 1.0 & 2.2 & 4.0 & 6.1 \\ 5 & 0 & 0 & 0 & (*) & .22 & .55 & 1.0 & 2.1 \\ 10 & 0 & 0 & 0 & (*) & .12 & .30 & .46 & 1.0\end{array}$

* Less than $0.05 \mathrm{ft}^{3} / \mathrm{s}$. 
COASTAL AREA FROM TAMPA BAY TO WITHLACOOCHEE RIVER

02310750 Crystal River near Crystal River Fla.

LOCATION.--Lat $28^{\circ} 54^{\prime} 17^{\prime \prime}$, long $82^{\circ} 38^{\prime} 13^{\prime \prime}$, in SE $\frac{1}{4}$ sec.13, T.18 S., R.16 E., Citrus County, Hydrologic Unit 03100207, at left bank, 0.1 mi upstream from Salt River, 2.7 mi west of town of Crystal River, and $4.0 \mathrm{mi}$ upstream from mouth.

DRAINAGE AREA.---Indeterminate.

PERIOD OF RECORD ANALYZED.--July 1964 to June 1977.

REMARKS.--Flow affected by tide. Negative flow at times; negative discharges treated as zero values.

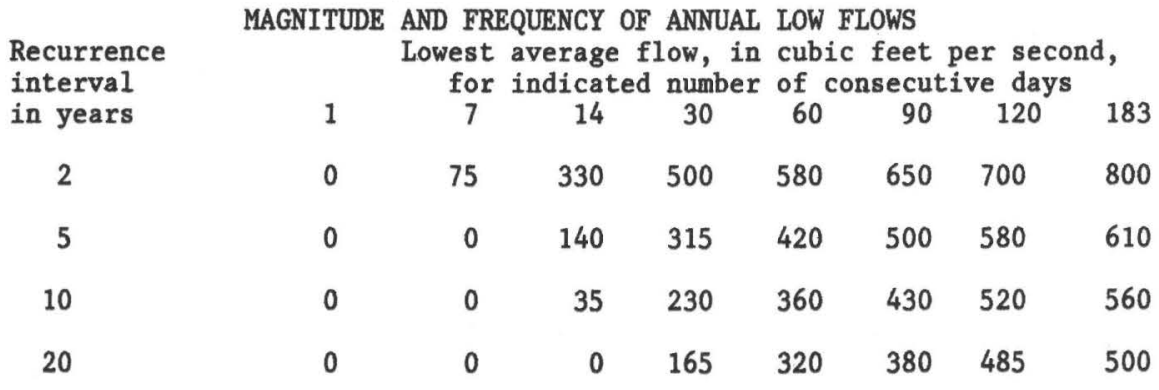


LOCATION.--Lat $28^{\circ} 21^{\prime} 38^{\prime \prime}$, long $81^{\circ} 49^{\prime} 08^{\prime \prime}$, in NW/ sec.33, T.24 S., R.25 E., Polk County, Hydrologic Unit 03100208 , near center of span on upstream side of bridge on State Highway 33, $2.5 \mathrm{mi}$ north of Eva, $6.5 \mathrm{mi}$ upstream from small tributary, and $138 \mathrm{mi}$ upstream from mouth.

DRAINAGE AREA. --130 $\mathrm{mi}^{2}$, approximately.

PERIOD OF RECORD ANALYZED.--July 1959 to June 1977.

\begin{tabular}{ccccccccr}
$\begin{array}{l}\text { Recurrence } \\
\text { interval }\end{array}$ & MAGNITUDE AND FREQUENCY OF ANNUAL LOW FLOWS \\
in years & \multicolumn{7}{c}{$\begin{array}{c}\text { Lowest average flow, in cubic feet per second, } \\
\text { for indicated number of consecutive days }\end{array}$} \\
2 & 1 & 7 & 14 & 30 & 60 & 90 & 120 & 183 \\
2 & 0 & 0 & $(*)$ & 0.09 & 0.74 & 1.9 & 5.0 & 9.9 \\
5 & 0 & 0 & 0 & 0 & .09 & .39 & 1.0 & 2.1 \\
10 & 0 & 0 & 0 & 0 & $(*)$ & .18 & .43 & .88 \\
20 & 0 & 0 & 0 & 0 & 0 & .09 & .20 & .41
\end{tabular}

02310947 Withlacoochee River near Cumpressco, Fla.

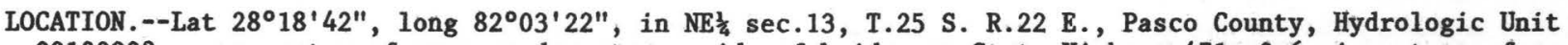
03100208 , near center of span on downstream side of bridge on State Highway $471,0.6 \mathrm{mi}$ upstream from Gator Creek, $3.4 \mathrm{mi}$ south of Cumpressco, $5.8 \mathrm{mi}$ east of Richland, and $120 \mathrm{mi}$ upstream from mouth.

DRAINAGE AREA. --280 $\mathrm{mi}^{2}$, approximately.

PERIOD OF RECORD ANALYZED.--July 1967 to June 1977.

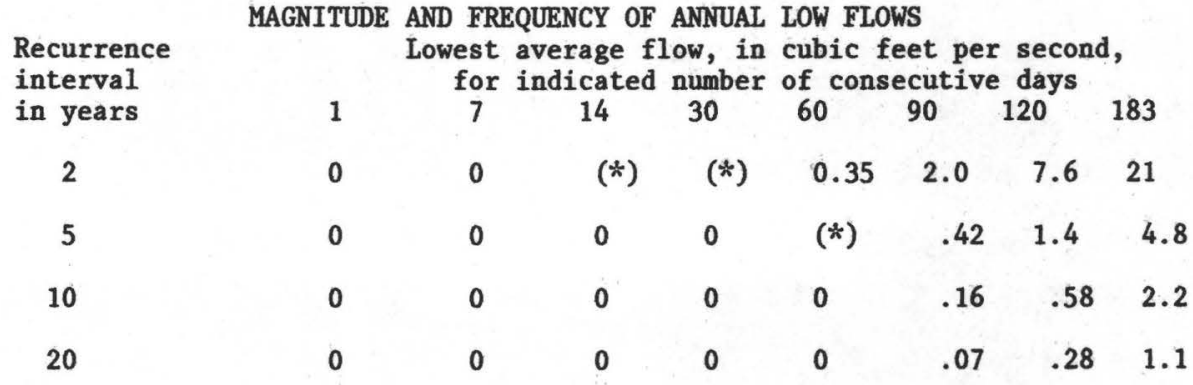

* Less than $0.05 \mathrm{ft}^{3} / \mathrm{s}$. 
LOCATION.--Lat $28^{\circ} 28^{\prime} 47^{\prime \prime}$, long $82^{\circ} 10^{\prime} 40^{\prime \prime}$, in SE多 sec.14, T.23 S., R.21 E., Hernando County, Hydrologic Unit 03100208 , on right bank at downstream side of bridge on U.S. Highway 301, 1.6 mi northeast of Trilby, 10 mi upstream from Little Withlacoochee River, and 93 mi upstream from mouth.

DRAINAGE AREA. $--570 \mathrm{mi}^{2}$, approximately.

PERIOD OF RECORD ANALYZED.--July 1930 to June 1977.

REMARKS.--Records include considerable amount of waste water diverted from ground-water supplies during packing season by Evans and Pasco Packing Companies, in SW sec.23, T.24 S., R.21 E., 5 mi upstream from Withlacoochee River.

\begin{tabular}{|c|c|c|c|c|c|c|c|c|}
\hline $\begin{array}{l}\text { Recurrence } \\
\text { interval }\end{array}$ & MAGNITUDE & $\begin{array}{l}\text { ND FRE } \\
\text { owest } \\
\text { for }\end{array}$ & $\begin{array}{l}\text { NCY OF } \\
\text { rage f } \\
\text { icated }\end{array}$ & $\begin{array}{l}\text { ANNUAL } \\
\text { ow, in } \\
\text { number }\end{array}$ & $\begin{array}{l}\text { LOW } \\
\text { cubi } \\
\text { of }\end{array}$ & $\begin{array}{l}\text { WS } \\
\text { eet } \\
\text { ecut }\end{array}$ & $\begin{array}{l}\text { r sec } \\
\text { e day }\end{array}$ & \\
\hline in years & 1 & 7 & 14 & 30 & 60 & 90 & 120 & 183 \\
\hline 2 & 26 & 28 & 31 & 37 & 48 & 64 & 83 & 121 \\
\hline 5 & 15 & 16 & 18 & 21 & 26 & 33 & 41 & 60 \\
\hline 10 & 11 & 12 & 13 & 16 & 19 & 23 & 29 & 42 \\
\hline 20 & 8.8 & 9.8 & 11 & 13 & 14 & 17 & 21 & 32 \\
\hline 30 & 7.8 & 8.7 & 9.6 & 11 & 12 & 14 & 18 & 27 \\
\hline 50 & 6.9 & 7.6 & 8.2 & 9.8 & 11 & 12 & 15 & 23 \\
\hline
\end{tabular}

LOCATION.--Lat $28^{\circ} 31^{\prime} 17^{\prime \prime}$, long $82^{\circ} 03^{\prime} 18^{\prime \prime}$, in NE $\frac{1}{4}$ sec.1, T.23 S., R.22 E., Sumter County, Hydrologic Unit 03100208 , near center of span on downstream side of bridge on State Highway $471,2.3$ mi south of Tarrytown, $3.1 \mathrm{mi}$ southwest of Linden, and $14 \mathrm{mi}$ upstream from mouth.

DRAINAGE AREA.--85 $\mathrm{mi}^{2}$, approximately.

PERIOD OF RECORD ANALYZED.--July 1967 to June 1977.

Recurrence
interval
in years
2
5
10
20

MAGNITUDE AND FREQUENCY OF ANNUAL LOW FLOWS Lowest average flow, in cubic feet per second, for indicated number of consecutive days

* Less than $0.05 \mathrm{ft}^{3} / \mathrm{s}$. 
LOCATION.--Lat $28^{\circ} 34^{\prime} 21^{\prime \prime}$, long $82^{\circ} 09^{\prime} 20^{\prime \prime}$, in SE⿺ sec.13, T.22 S., R.21 E., Hernando County, Hydrologic Unit 03100208 , near center of span on downstream side of bridge on U.S. Highway $301,0.2$ mi north of Rerdell and $4.8 \mathrm{mi}$ upstream from mouth.

DRAINAGE AREA. --145 $\mathrm{mi}^{2}$, approximately.

PERIOD OF RECORD ANALYZED.--July 1959 to June 1977.

\begin{tabular}{|c|c|c|c|c|c|c|c|c|}
\hline \multirow{2}{*}{$\begin{array}{l}\text { Recurrence } \\
\text { interval } \\
\text { in years }\end{array}$} & \multicolumn{4}{|c|}{$\begin{array}{l}\text { MAGNITUDE AND FREQUENCY OF ANNUAL } \\
\text { Lowest average flow, in } \\
\text { for indicated number }\end{array}$} & \multicolumn{4}{|c|}{$\begin{array}{l}\text { LOW FLOWS } \\
\text { cubic feet per second, } \\
\text { of consecutive days }\end{array}$} \\
\hline & 1 & 7 & 14 & 30 & 60 & 90 & 120 & 183 \\
\hline 2 & 1.5 & 2.3 & 2.8 & 3.0 & 4.8 & 9.3 & 15 & 24 \\
\hline 5 & .10 & .20 & .32 & .50 & 1.2 & 2.4 & 4.5 & 7.6 \\
\hline 10 & 0 & (*) & $(*)$ & .05 & .20 & .85 & 2.2 & 4.0 \\
\hline 20 & 0 & 0 & 0 & 0 & 0 & .32 & 1.1 & 2.3 \\
\hline
\end{tabular}

02312500 Withlacoochee River at Croom, Fla.

LOCATION.--Lat $28^{\circ} 35^{\prime} 3^{\prime \prime}$, long $82^{\circ} 13^{\prime} 20^{\prime \prime}$, in NE $\frac{1}{4}$ sec.8, T.22 S., R.21 E., Hernando County, Hydrologic Unit 03100208 , on left.bank at upstream side of abandoned highway bridge, 0.4 mi northwest of Croom, $2.3 \mathrm{mi}$ downstream from Little Withlacoochee River, $4.5 \mathrm{mi}$ southeast of Nobleton, and $77 \mathrm{mi}$ upstream from mouth.

DRAINAGE AREA. $--810 \mathrm{mi}^{2}$, approximately.

PERIOD OF RECORD ANALYZED.--July 1940 to June 1977.

REMARKS.--Records include considerable amount of waste water diverted from ground-water supplies during packing season by packing companies upstream (see sta 02312000 Withlacoochee River at Trilby).

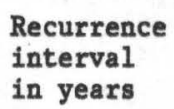

2

5

10

20

30

50
MAGNITUDE AND FREQUENCY OF ANNUAL LOW FLOWS

Lowest average flow, in cubic feet per second, for indicated number of consecutive days

1

$7 \quad 1$

66

70

73

30

60

$90 \quad 120$

183

39

41

43

47

93

$116 \quad 140$

191

29

31

33

36

55

$\begin{array}{ll}66 & 79\end{array}$

107

20

22

25

27

33

4960

81

17

18

21

23

29

39

47

65

15

$16 \quad 18$

20

26

34

41

57

30

37

51

* Less than $0.05 \mathrm{ft}^{3} / \mathrm{s}$. 


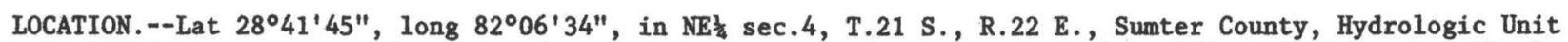
03100208 , near center of span on downstream side of bridge on State Highway $475,2.2$ mi north of Bushnell and $10 \mathrm{mi}$ upstream from mouth.

DRAINAGE AREA. $--40 \mathrm{mi}^{2}$, approximately.

PERIOD OF RECORD ANALYZED.--July 1964 to June 1977.

\begin{tabular}{|c|c|c|c|c|c|c|c|c|}
\hline \multirow{2}{*}{$\begin{array}{l}\text { Recurrence } \\
\text { interval } \\
\text { in years }\end{array}$} & \multirow{2}{*}{$\begin{array}{c}\text { MAGNITUDE } \\
1\end{array}$} & $\begin{array}{l}\text { D FREC } \\
\text { owest a } \\
\text { for }\end{array}$ & $\begin{array}{l}\text { NCY of } \\
\text { rage } t \\
\text { icated }\end{array}$ & $\begin{array}{l}\text { ANNUAL } \\
\text { ow, in } \\
\text { number }\end{array}$ & $\begin{array}{l}\text { LOW FI } \\
\text { cubic } \\
\text { of cor }\end{array}$ & & $\begin{array}{l}r \text { sec } \\
\text { e day }\end{array}$ & \\
\hline & & 7 & 14 & 30 & 60 & 90 & 120 & 183 \\
\hline 2 & 9.9 & 13 & 14 & 15 & 16 & 17 & 19 & 20 \\
\hline 5 & 7.8 & 10 & 11 & 11 & 13 & 13 & 14 & 15 \\
\hline 10 & 6.4 & 8.4 & 9.0 & 9.4 & 11 & 11 & 13 & 13 \\
\hline 20 & 5.5 & 7.2 & 7.8 & 8.0 & 9.4 & 10 & 11 & 12 \\
\hline
\end{tabular}

02312700 Outlet River at Panacoochee Retreats, Fla.

LOCATION.--Lat $28^{\circ} 49^{\prime} 01^{\prime \prime}$, long $82^{\circ} 08^{\prime} 40^{\prime \prime}$, in SE $\frac{1}{4}$ sec.19, T.19 S., R.22 E., Sumter County, Hydrologic Unit 03100208 , on west shore of Lake Panasoffkee, $0.8 \mathrm{mi}$ north of outlet, $1.3 \mathrm{mi}$ north of Panacoochee Retreats, $2.0 \mathrm{mi}$ upstream from mouth, and $5.1 \mathrm{mi}$ northwest of town of Lake Panasoffkee.

DRAINAGE AREA. $--420 \mathrm{mi}^{2}$, approximately.

PERIOD OF RECORD ANALYZED.--July 1964 to June 1977.

Recurrence
interval
in years
2
5
10
20

20
MAGNITUDE AND FREQUENCY OF ANNUAL LOW FLOWS Lowest average flow, in cubic feet per second, for indicated number of consecutive days

\begin{tabular}{rrrrrrrrr} 
& \multicolumn{2}{c}{ for } & indicated number & of consecutive days \\
1 & 7 & 14 & 30 & 60 & 90 & 120 & 183 \\
99 & 102 & 105 & 110 & 117 & 130 & 141 & 155 \\
52 & 62 & 67 & 78 & 87 & 100 & 111 & 124 \\
34 & 44 & 51 & 62 & 72 & 83 & 96 & 107 \\
23 & 32 & 39 & 50 & 60 & 70 & 84 & 90
\end{tabular}


LOCATION.--Lat $28^{\circ} 49^{\prime} 23^{\prime \prime}$, long $82^{\circ} 11^{\prime} 00^{\prime \prime}$, in NW/ sec.23, T.19 S., R.21 E., Sumter County, Hydrologic Unit 03100208 , at downstream end of left wall of lock of Wysong Dam, at Carlson, 1.8 mi downstream from outlet River, $2.7 \mathrm{mi}$ southeast of Rutland, and $55 \mathrm{mi}$ upstream from mouth.

DRAINAGE AREA. $--1,520 \mathrm{mi}^{2}$, approximately .

PERIOD OF RECORD ANALYZED.--July 1966 to June 1977.

REMARKS.--Since August 1965, some regulation at station by manipulation of inflatable fabri-dam. Some diversions above station at times into Tsala Apopka Lake. Data adjusted by use of a flow correlation analysis with gaging station 02312500 , Withlacoochee River at Croom.

\begin{tabular}{|c|c|c|c|c|c|c|c|c|}
\hline $\begin{array}{l}\text { Recurrence } \\
\text { interval }\end{array}$ & MAGNITU & $\begin{array}{l}\text { AND FF } \\
\text { Lowest } \\
\text { for }\end{array}$ & $\begin{array}{l}\text { QUENCY } \\
\text { average } \\
\text { indicat }\end{array}$ & $\begin{array}{l}\text { ANNUAL } \\
\text { low, in } \\
\text { number }\end{array}$ & $\begin{array}{l}\text { LOW } \\
\text { cubi } \\
\text { of }\end{array}$ & $\begin{array}{l}\text { cows } \\
\text { feet } \\
\text { ssecut }\end{array}$ & $\begin{array}{l}\text { r sed } \\
\text { e day }\end{array}$ & \\
\hline in years & 1 & 7 & 14 & 30 & 60 & 90 & 120 & 183 \\
\hline 2 & 197 & 203 & 208 & 216 & 235 & 264 & 291 & 342 \\
\hline 5 & 150 & 154 & 158 & 165 & 179 & 197 & 216 & 253 \\
\hline 10 & 128 & 133 & 137 & 144 & 156 & 169 & 187 & 219 \\
\hline 20 & 106 & 111 & 119 & 124 & 137 & 150 & 165 & 195 \\
\hline
\end{tabular}

02313000 Withlacoochee River near Holder, Fla.

LOCATION.--Lat $28^{\circ} 59^{\prime} 19^{\prime \prime}$, long $82^{\circ} 20^{\prime} 59^{\prime \prime}$, in NW/ sec.30, T.17 S., R.20 E., Marion County, Hydrologic Unit 03100208 , near right bank on downstream side of bridge on State Highway $200,4.5$ mi northeast of Holder and $38 \mathrm{mi}$ upstream from mouth.

DRAINAGE AREA. $--1,825 \mathrm{mi}^{2}$, approximately.

PERIOD OF RECORD ANALYZED.--July 1932 to June 1977.

\begin{tabular}{|c|c|c|c|c|c|c|c|c|}
\hline $\begin{array}{l}\text { Recurrence } \\
\text { interval }\end{array}$ & MAGNITL & $\begin{array}{l}\text { ND FR } \\
\text { owest } \\
\text { for }\end{array}$ & $\begin{array}{l}\text { QUENCY } \\
\text { average } \\
\text { indicat }\end{array}$ & $\begin{array}{l}\text { ANNUAL } \\
\text { flow, in } \\
\text { number }\end{array}$ & $\begin{array}{l}\text { LOW } \\
\text { cubi } \\
\text { of }\end{array}$ & $\begin{array}{l}\text { LOWS } \\
\text { feet } \\
\text { isecut }\end{array}$ & $\begin{array}{l}\text { er sec } \\
\text { ve day }\end{array}$ & \\
\hline in years & 1 & 7 & 14 & 30 & 60 & 90 & 120 & 183 \\
\hline 2 & 340 & 360 & 370 & 390 & 440 & 490 & 550 & 660 \\
\hline 5 & 180 & 198 & 229 & 249 & 260 & 295 & 330 & 400 \\
\hline 10 & 150 & 158 & 183 & 198 & 200 & 240 & 270 & 340 \\
\hline 20 & 130 & 134 & 151 & 164 & 170 & 190 & 230 & 290 \\
\hline 30 & 120 & 123 & 136 & 142 & 155 & 177 & 210 & 275 \\
\hline 50 & 110 & 112 & 122 & 131 & 140 & 165 & 190 & 260 \\
\hline
\end{tabular}


LOCATION.--Lat $29^{\circ} 06^{\prime} 08^{\prime \prime}$, long $82^{\circ} 26^{\prime} 16^{\prime \prime}$, in SE交 sec.12, T.16 S., R.18 E., Marion County, Hydrologic Unit 03100208 , at head of springs, $3.9 \mathrm{mi}$ north of Dunnellon and $5.7 \mathrm{mi}$ upstream from mouth.

DRAINAGE AREA. --Indeterminate.

PERIOD OF RECORD ANALYZED.--July 1965 to June 1977.

REMARKS.--Data adjusted by use of a flow correlation analysis with gaging station 02239500, Silver Springs near Ocala.

\begin{tabular}{|c|c|c|c|c|c|c|c|c|}
\hline \multirow{2}{*}{$\begin{array}{l}\text { Recurrence } \\
\text { interval } \\
\text { in years }\end{array}$} & \multicolumn{2}{|c|}{ Lowest } & $\begin{array}{l}\text { Terage } \\
\text { dicat }\end{array}$ & $\begin{array}{l}\text { low, } \\
\text { numt }\end{array}$ & cubi & $\begin{array}{l}\text { feet } \\
\text { secut }\end{array}$ & \multicolumn{2}{|c|}{$\begin{array}{l}\text { second, } \\
\text { days }\end{array}$} \\
\hline & 1 & 7 & 14 & 30 & 60 & 90 & 120 & 183 \\
\hline 2 & 618 & 621 & 623 & 628 & 636 & 648 & 659 & 67 \\
\hline 5 & 565 & 568 & 568 & 572 & 579 & 587 & 595 & 60 \\
\hline 10 & 538 & 541 & 542 & 545 & 551 & 558 & 564 & \\
\hline 20 & 518 & 520 & 522 & 525 & 530 & 535 & 539 & \\
\hline
\end{tabular}




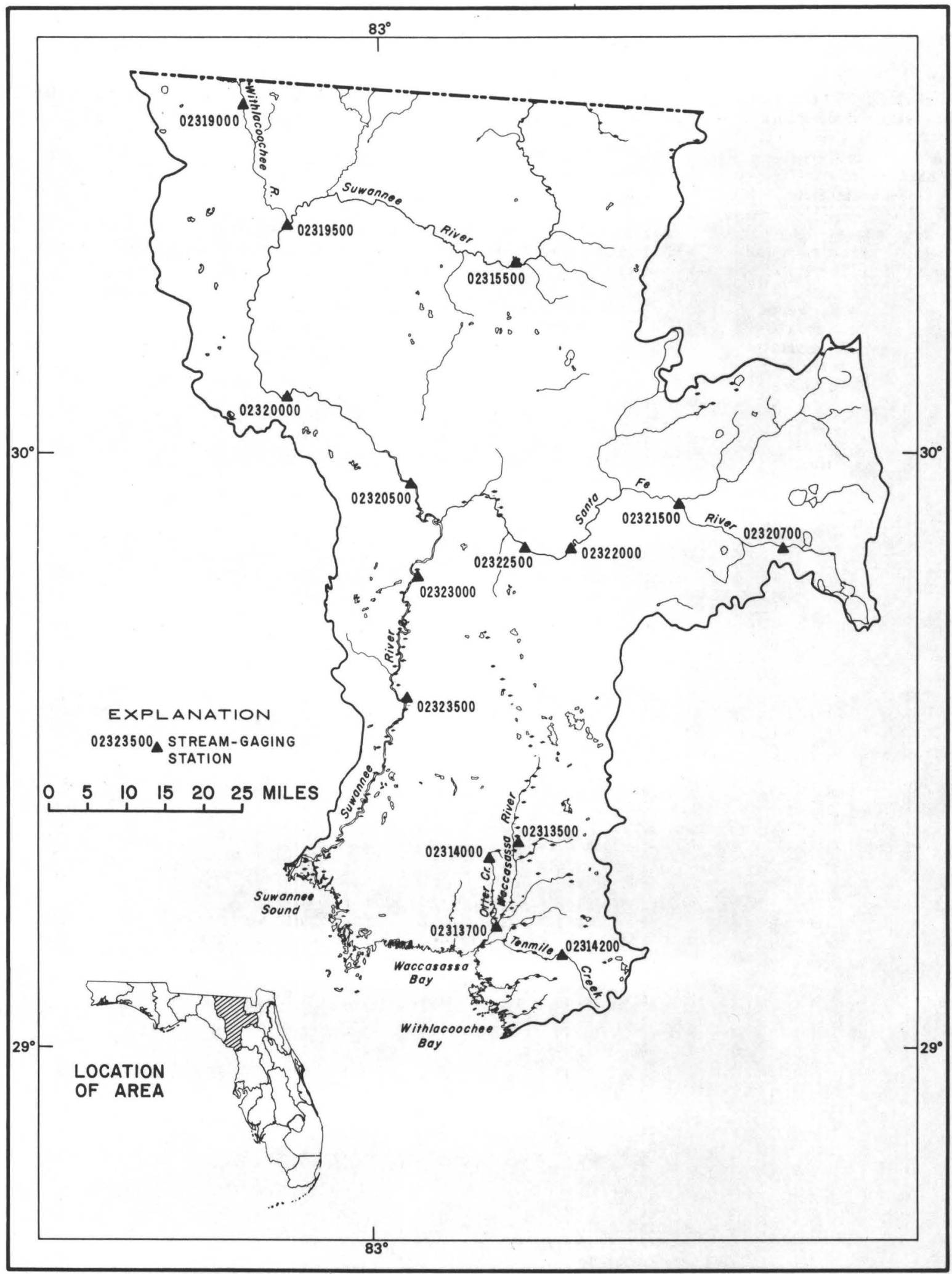

Figure 12.--Location of stream-gaging stations in the Waccasassa and Suwannee River basins. 
LOCATION.--Lat $29^{\circ} 12^{\prime} 14^{\prime \prime}$, long $82^{\circ} 46^{\prime} 09^{\prime \prime}$, in SW sec.2, T.15 S., R.15 E., Levy County, Hydrologic Unit 03110101 , near right bank at abandoned railroad grade, 0.5 mi upstream from Otter Creek, $3.6 \mathrm{mi}$ upstream from mouth, and $4 \mathrm{mi}$ southwest of Gulf Hammock.

DRAINAGE AREA.--480 $\mathrm{mi}^{2}$, approximately, including that of Otter Creek.

PERIOD OF RECORD ANALYZED.--July 1964 to June 1977.

REMARKS.--Flow affected by tide. Negative flow at times. Negative discharges treated as zero values. Records include the flow of Otter Creek.

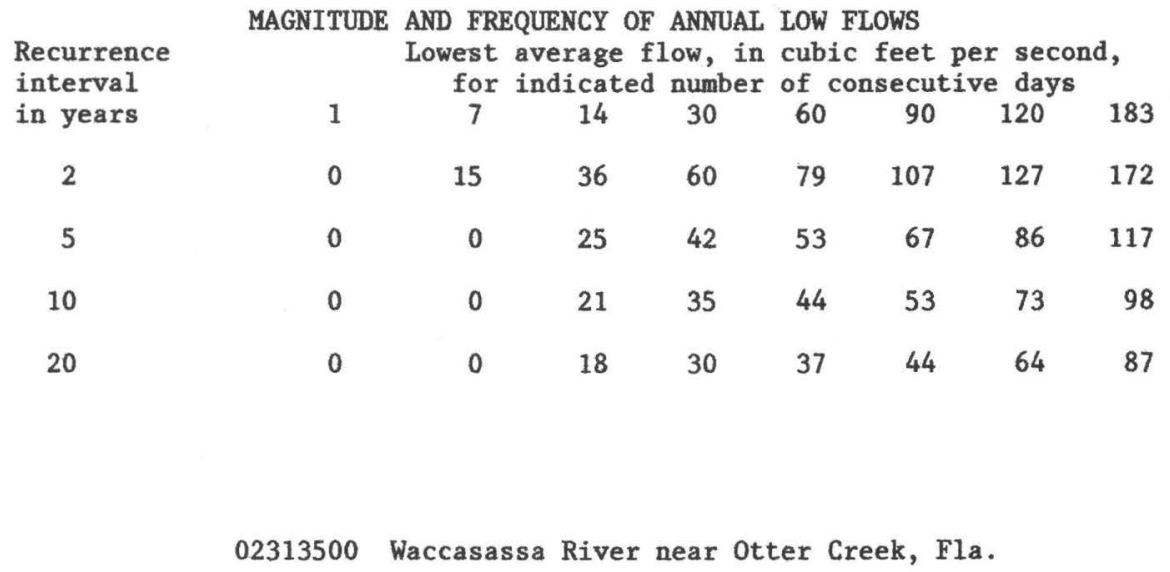

LOCATION.--Lat $29^{\circ} 21^{\prime} 15^{\prime \prime}$, long $82^{\circ} 44^{\prime} 06^{\prime \prime}$, in NW/ sec.17, T.13 S., R.16 E., Levy County, Hydrologic Unit 03110101, near right bank at upstream side of bridge on State Highway 24, 3.0 mi northeast of village of Otter Creek, and 14.0 mi upstream from mouth of Otter Creek.

DRAINAGE AREA.--Indeterminate. The combined drainage area of Waccasassa River near Otter Creek and Otter Creek (station 02314000) is $300 \mathrm{mi}^{2}$, approximately.

PERIOD OF RECORD ANALYZED. --July 1946 to June 1953.

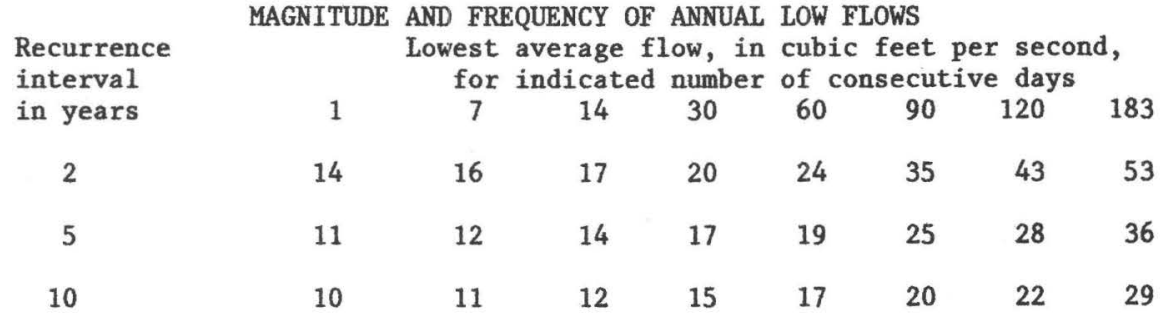


LOCATION.--Lat $29^{\circ} 19^{\prime} 08^{\prime \prime}$, long $82^{\circ} 47^{\prime} 03^{\prime \prime}$, in SW sec. 26 , T.13 S., R.15 E., Levy County, Hydrologic Unit 03110101 , near right bank at upstream side of bridge on State Highway $24,0.8$ mi southwest of village of Otter Creek, $1.1 \mathrm{mi}$ southwest of U.S. Highway 98 , and $9.7 \mathrm{mi}$ upstream from mouth.

DRAINAGE AREA.--Indeterminate. The combined drainage area of Otter Creek near 0tter Creek and Waccasassa River near Otter Creek (station 02313500) is $300 \mathrm{mi}^{2}$, approximately.

PERIOD OF RECORD ANALYZED.--July 1945 to June 1953.

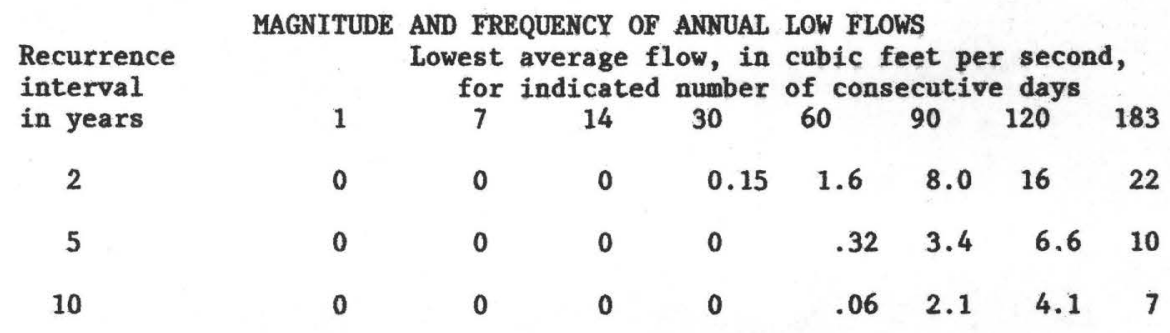

02314200 Tenmile Creek at Lebanon Station, Fla.

LOCATION.--Lat $29^{\circ} 09^{\prime} 39^{\prime \prime}$, long $82^{\circ} 38^{\prime} 21^{\prime \prime}$, in SE考 sec.24, T.15 S., R.16 E., Levy County, Hydrologic Unit 03110101 , near center of span on downstream side of bridge on U.S. Highway 19 and 98 , just downstream from North Prong Tenmile Creek, $0.2 \mathrm{mi}$ south of Lebanon Station, $9.4 \mathrm{mi}$ upstream from mouth, and $13 \mathrm{mi}$ northwest of Dunnellon.

DRAINAGE AREA.--26 $\mathrm{mi}^{2}$, approximately; $34 \mathrm{mi}^{2}$, approximately, including that of Horse Hole Creek.

PERIOD OF RECORD ANALYZED.--July 1964 to June 1978.

REMARKS.--Records do not include considerable amount of water diverted naturally above station into Horse Hole Creek basin.

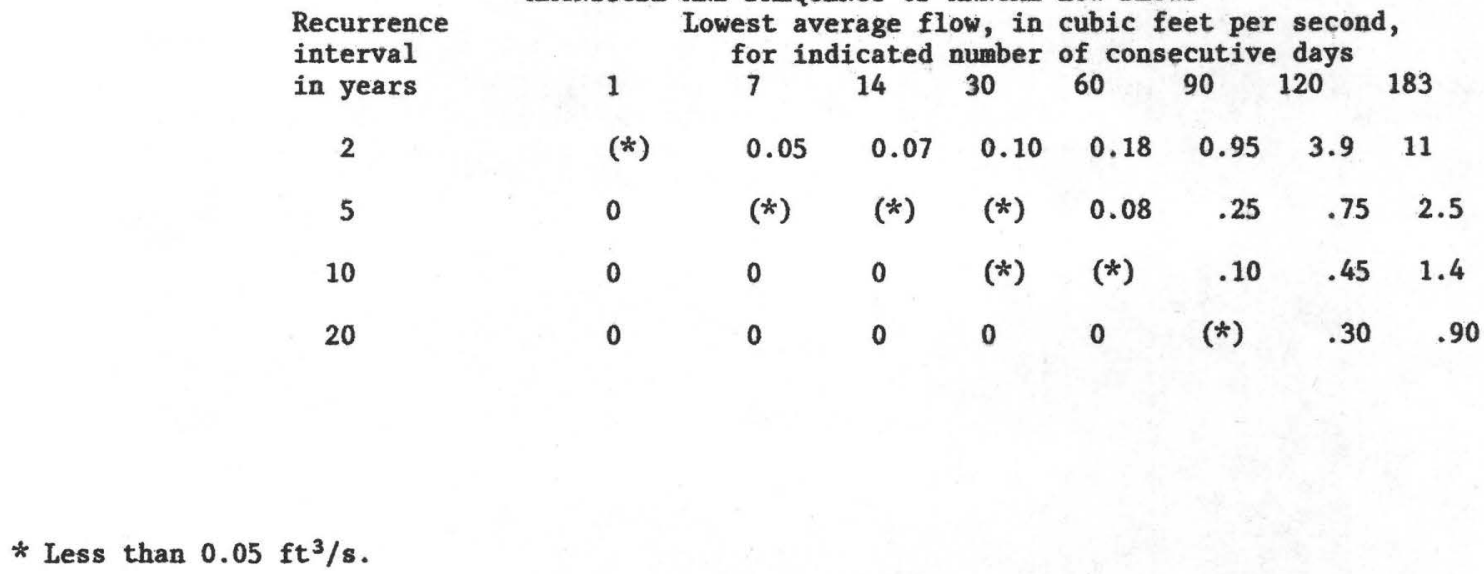

MAGNITUDE AND FREQUENCY OF ANNUAL LOW FLOWS 
LOCATION.--Lat $30^{\circ} 19^{\prime} 32^{\prime \prime}$, long $82^{\circ} 44^{\prime} 18^{\prime \prime}$, in SW/ sec.8, T.2 S., R.16 E., Columbia County, Hydrologic Unit 03110201 , on left bank at downstream side of bridge on U.S. Highway 41, 1.0 mi southeast of White Springs and 171 mi upstream from mouth.

DRAINAGE AREA.--2,430 $\mathrm{mi}^{2}$, approximately, includes part of watershed in Okefenokee Swamp which is indeterminate. PERIOD OF RECORD ANALYZED.--April 1907 to March 1908, April 1927 to March 1978.

\begin{tabular}{|c|c|c|c|c|c|c|c|c|}
\hline $\begin{array}{l}\text { Recurrence } \\
\text { interval }\end{array}$ & MAGNITUD & $\begin{array}{l}\text { ID FREC } \\
\text { owest } \\
\text { for }\end{array}$ & $\begin{array}{l}\text { rage } \\
\text { ragated } \\
\text { icate }\end{array}$ & $\begin{array}{l}\text { ANNUAL } \\
\text { low, in } \\
\text { number }\end{array}$ & $\begin{array}{l}\text { LOW FI } \\
\text { cubic } \\
\text { of cor }\end{array}$ & $\begin{array}{l}\text { WS } \\
\text { Eeet p } \\
\text { secuti }\end{array}$ & $\begin{array}{l}\text { secor } \\
\text { e days }\end{array}$ & \\
\hline in years & 1 & 7 & 14 & 30 & 60 & 90 & 120 & 183 \\
\hline 2 & 75 & 80 & 88 & 120 & 160 & 240 & 365 & 770 \\
\hline 5 & 15 & 15 & 17 & 22 & 34 & 56 & 84 & 190 \\
\hline 10 & 9.2 & 10 & 11 & 13 & 17 & 25 & 38 & 8 \\
\hline 20 & 6.7 & 7.4 & 8.2 & 10 & 11 & 15 & 20 & 3 \\
\hline 30 & 5.8 & 6.3 & 7.0 & 8.6 & 9.7 & 12 & 15 & 2 \\
\hline 50 & 4.8 & 5.2 & 5.7 & 7.4 & 8.5 & 9.0 & 9.5 & 16 \\
\hline
\end{tabular}

02319000 Withlacoochee River near Pinetta, Fla.

LOCATION.--Lat $30^{\circ} 35^{\prime} 43^{\prime \prime}$, long $83^{\circ} 15^{\prime} 35^{\prime \prime}$, in NW/ sec.7, T.2 N., R.11 E., Madison County, Hydrologic Unit 03110203 , on right bank $30 \mathrm{ft}$ downstream from bridge, $0.1 \mathrm{mi}$ downstream from small tributary, $0.3 \mathrm{mi}$ west of Bellville, $5.6 \mathrm{mi}$ east of Pinetta, and $22 \mathrm{mi}$ upstream from mouth.

DRAINAGE AREA. $--2,120 \mathrm{mi}^{2}$, approximately.

PERIOD OF RECORD ANALYZED.--April 1932 to March 1978.

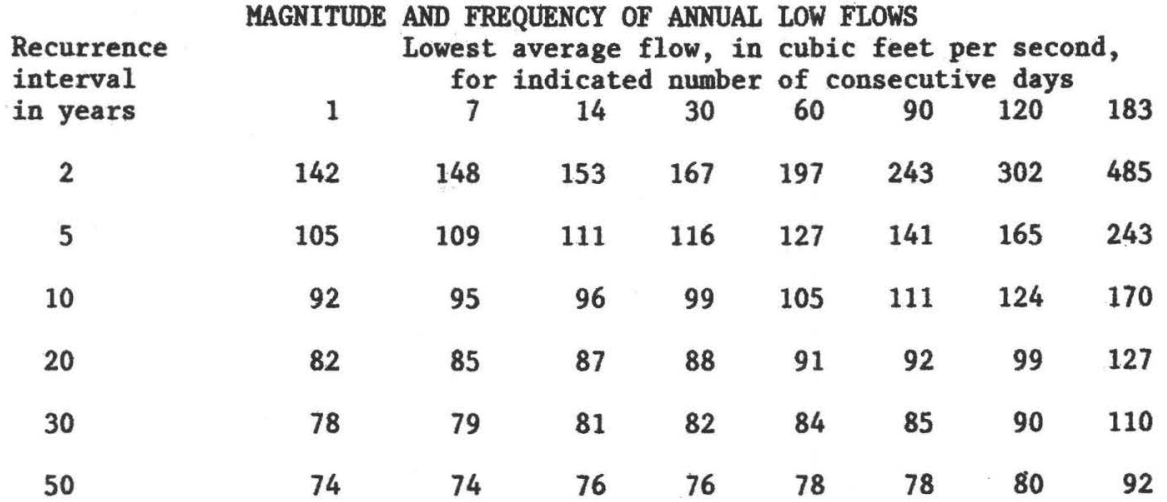


LOCATION.--Lat $30^{\circ} 23^{\prime} 04^{\prime \prime}$, long $83^{\circ} 10^{\prime} 19^{\prime \prime}$, in NEz sec.24, T.1 S., R.11 E., Suwannee County, Hydrologic Unit 03110205, on left bank at Bllaville, $100 \mathrm{ft}$ upstream from Seaboard Air Line Railroad bridge, $200 \mathrm{ft}$ downstream from Withlacoochee River, $900 \mathrm{ft}$ upstream from bridge on U.S. Highway 90, and 127 mi upstream from mouth.

DRAINAGE AREA.- -6,970 $\mathrm{mi}^{2}$, approximately, includes part of watershed in Okefenokee Swamp which is indeterminate. PERIOD OF RECORD ANALYZED.--April 1927 to March 1978.

\begin{tabular}{|c|c|c|c|c|c|c|c|c|}
\hline $\begin{array}{l}\text { Recurrence } \\
\text { interval }\end{array}$ & MAGNITUI & $\begin{array}{l}\text { AND FR } \\
\text { Lowest } \\
\text { for }\end{array}$ & $\begin{array}{l}\text { QUENCY } \\
\text { average } \\
\text { indicat }\end{array}$ & $\begin{array}{l}\text { F ANDL } \\
\text { flow, } \\
\text { d numb }\end{array}$ & $\begin{array}{l}\text { I } \text { IOW } \\
\text { n cubi } \\
r \text { of }\end{array}$ & $\begin{array}{l}\text { Lows } \\
\text { feet } \\
\text { nsecut }\end{array}$ & $\begin{array}{l}\text { er se } \\
\text { ve day }\end{array}$ & ad, \\
\hline in years & 1 & 7 & 14 & 30 & 60 & 90 & 120 & 183 \\
\hline 2 & 1680 & 1700 & 1740 & 1830 & 2020 & 2240 & 2510 & 3250 \\
\hline 5 & 1220 & 1230 & 1250 & 1280 & 1360 & 1470 & 1610 & 2000 \\
\hline 10 & 1050 & 1060 & 1070 & 1080 & 1130 & 1200 & 1300 & 1570 \\
\hline 20 & 936 & 946 & 952 & 960 & 976 & 1030 & 1110 & 1290 \\
\hline 30 & 880 & 890 & 900 & 905 & 915 & 950 & 1020 & 1170 \\
\hline 50 & 831 & 840 & 844 & 850 & 860 & 880 & 933 & 1050 \\
\hline
\end{tabular}

02320000 Suwannee River at Luraville, Fla.

LOCATION.--Iat $30^{\circ} 06^{\prime}$, long $83^{\circ} 10^{\prime}$, in sec.30, T.4 S., R.12 E., Suwannee County, Hydrologic Unit 02319500, at highway bridge $1 \mathrm{mi}$ south of Luraville and $3 \mathrm{mi}$ upstream from Grants Ferry Shoals. A large spring discharges into river $500 \mathrm{ft}$ upstream from gage on left bank.

DRAINAGE AREA. $--7,330 \mathrm{mi}^{2}$, approximately

PERIOD OF RECORD ANALYZED.--April 1927 to March 1937.

REMARKS.--Data adjusted by use of a flow correlation analysis with gaging station 02319500, Suwannee River at Ellaville.

Recurrence interval

in years

2

5

10

20

MAGNITUDE AND FREQUENCY OF ANNUAL LOW FLOWS

Lowest average flow, in cubic feet per second, for indicated number of consecutive days

$\begin{array}{rrrrrrrr}1 & 7 & 14 & 30 & 60 & 90 & 120 & 183 \\ 120 & 2140 & 2190 & 2300 & 2520 & 2780 & 3090 & 3940 \\ 1570 & 1580 & 1610 & 1640 & 1740 & 1870 & 2040 & 2500 \\ 360 & 1370 & 1390 & 1400 & 1460 & 1540 & 1670 & 1990 \\ 120 & 1240 & 1240 & 1250 & 1270 & 1340 & 1440 & 1650\end{array}$




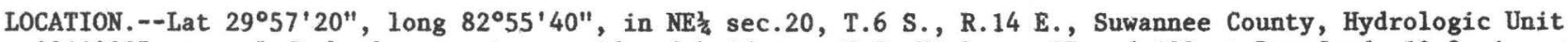
03110205 , near left bank on upstream side of bridge on U.S. Highways 27 and 129 at Branford, 10.8 mi upstream from Santa Fe River and 75 mi upstream from mouth.

DRAINAGE AREA. --7,880 $\mathrm{mi}^{2}$, includes part of watershed in Okefenokee Swamp which in indeterminate.

PERIOD OF RECORD ANALYZED.--April 1932 to March 1978.

\begin{tabular}{|c|c|c|c|c|c|c|c|c|}
\hline \multirow{2}{*}{$\begin{array}{l}\text { Recurrence } \\
\text { interval } \\
\text { in years }\end{array}$} & MAGNITUD & $\begin{array}{l}\text { AND FR } \\
\text { Lowest } \\
\text { for }\end{array}$ & $\begin{array}{l}\text { QUENCY } \\
\text { average } \\
\text { indicat }\end{array}$ & $\begin{array}{l}\text { F ANN } \\
\text { flow, } \\
\text { d numb }\end{array}$ & $\begin{array}{l}\text { L LOW } \\
\text { n cubi } \\
r \text { of }\end{array}$ & $\begin{array}{l}\text { Lows } \\
\text { feet } \\
\text { nsecu }\end{array}$ & $\begin{array}{l}\text { er sec } \\
\text { ve day }\end{array}$ & \\
\hline & 1 & 7 & 14 & 30 & 60 & 90 & $120^{\circ}$ & 183 \\
\hline 2 & 2600 & 2640 & 2670 & 2750 & 2950 & 3170 & 3420 & 4180 \\
\hline 5 & 2000 & 2030 & 2050 & 2080 & 2180 & 2290 & 2440 & 2870 \\
\hline 10 & 1770 & 1790 & 1800 & 1820 & 1880 & 1970 & 2080 & 2380 \\
\hline 20 & 1600 & 1620 & 1630 & 1650 & 1680 & 1750 & 1840 & 2040 \\
\hline 30 & 1520 & 1540 & 1550 & 1560 & 1580 & 1650 & 1730 & 1880 \\
\hline 50 & 1440 & 1460 & 1470 & 1480 & 1490 & 1550 & 1620 & 1730 \\
\hline
\end{tabular}

02320700 Santa Fe River near Graham, Fla.

LOCATION.--Lat $29^{\circ} 50^{\prime} 46^{\prime \prime}$, long $82^{\circ} 13^{\prime} 11^{\prime \prime}$, in NE多 sec.32 T.7 S., R.21 E., Alachua County, Hydrologic Unit 03110206, near left bank on upstream side of bridge on State Highway 225, $1.0 \mathrm{mi}$ south of Graham, $1.5 \mathrm{mi}$ upstream from Sampson River, and $71 \mathrm{mi}$ upstream from mouth.

DRAINAGE AREA. - $-94.9 \mathrm{mi}^{2}$.

PERIOD OF RECORD ANALYZED.--April 1958 to March 1978.

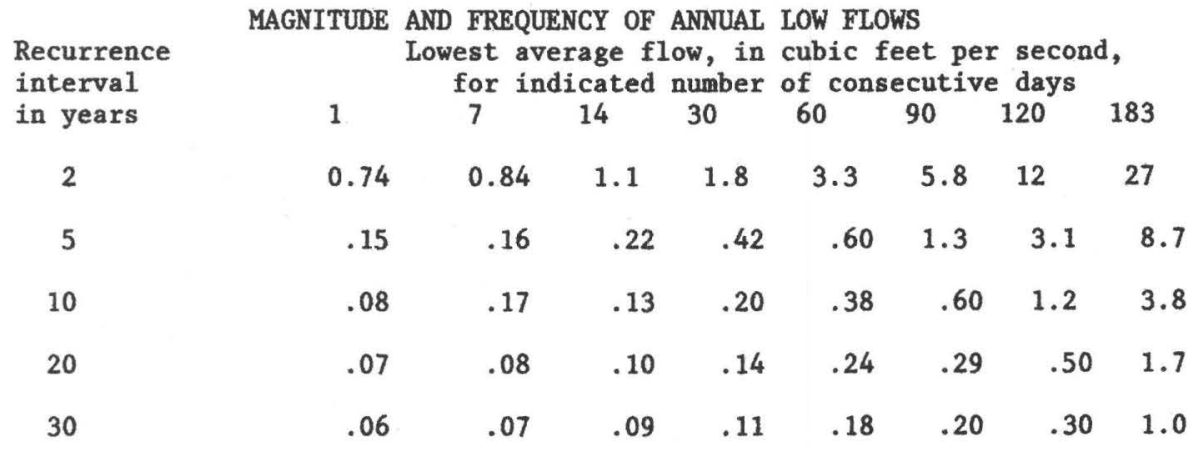


LOCATION.--Lat 29 $55^{\prime} 18^{\prime \prime}$, long $82^{\circ} 25^{\prime} 35^{\prime \prime}$, in SE $\frac{1}{4}$ sec.32, T.6 S., R.19 E., Alachua County, Hydrologic Unit 03110206 , near center of span on downstream side of bridge on State Highway $121,0.5$ mi south of Worthington Springs, $0.8 \mathrm{mi}$ downstream from New River, and $51 \mathrm{mi}$ upstream from mouth.

DRAINAGE AREA. $--575 \mathrm{mi}^{2}$.

PERIOD OF RECORD ANALYZED.--April 1932 to March 1979.

\begin{tabular}{|c|c|c|c|c|c|c|c|c|}
\hline $\begin{array}{l}\text { Recurrence } \\
\text { interval }\end{array}$ & MAGNITUDE & $\begin{array}{l}\text { D FREQ } \\
\text { west } \\
\text { for }\end{array}$ & $\begin{array}{l}\text { OF } \\
\text { cage } f \\
\text { icated }\end{array}$ & $\begin{array}{l}\text { ANNUAL } \\
\text { ow, in } \\
\text { number }\end{array}$ & $\begin{array}{l}\text { LOW FL } \\
\text { cubic } \\
\text { of con }\end{array}$ & $\begin{array}{l}\text { WS } \\
\text { eet pe } \\
\text { ecutiv }\end{array}$ & $\begin{array}{l}r \text { sec } \\
\text { e day }\end{array}$ & \\
\hline in years & 1 & 7 & 14 & 30 & 60 & 90 & 120 & 183 \\
\hline 2 & 10 & 12 & 15 & 20 & 32 & 51 & 86 & 197 \\
\hline 5 & 4.0 & 5.3 & 6.9 & 9.4 & 15 & 24 & 39 & 90 \\
\hline 10 & 2.3 & 3.3 & 4.4 & 5.8 & 9.8 & 16 & 25 & 58 \\
\hline 20 & 1.3 & 2.0 & 2.8 & 3.8 & 6.8 & 11 & 17 & 40 \\
\hline 30 & 1.0 & 1.5 & 2.2 & 3.2 & 5.2 & 9.0 & 14 & 32 \\
\hline 50 & .72 & 1.2 & 1.8 & 2.4 & 4.4 & 7.0 & 11 & 26 \\
\hline
\end{tabular}

02322000 Santa Fe River near High Springs, Fla.

LOCATION.--Lat $29^{\circ} 50^{\prime} 33^{\prime \prime}$, long $82^{\circ} 37^{\prime} 52^{\prime \prime}$, in NE光 sec.32, T.7 S., R. 17 E., Columbia County, Hydrologic Unit 03110206, near right bank at downstream side of bridge on U.S. Highway 27, 100 ft upstream from Seaboard Coast Line Railroad bridge, and 2 mi northwest of High Springs.

DRAINAGE AREA. --868 $\mathrm{mi}^{2}$, approximately.

PERIOD OF RECORD ANALYZED.--April 1931 to March 1971.

\begin{tabular}{|c|c|c|c|c|c|c|c|c|}
\hline $\begin{array}{l}\text { Recurrence } \\
\text { interval }\end{array}$ & MAGNITU & $\begin{array}{l}\text { ND FR } \\
\text { owest } \\
\text { for }\end{array}$ & $\begin{array}{l}\text { QUENCY } \\
\text { average } \\
\text { indicat }\end{array}$ & $\begin{array}{l}\text { ANNUAL } \\
\text { low, in } \\
\text { number }\end{array}$ & $\begin{array}{l}\text { LOW } \\
\text { cubi } \\
\text { of }\end{array}$ & $\begin{array}{l}\text { OWS } \\
\text { feet } \\
\text { secut }\end{array}$ & $\begin{array}{ll}\text { r sec } \\
\text { je day }\end{array}$ & \\
\hline in years & 1 & 7 & 14 & 30 & 60 & 90 & 120 & 183 \\
\hline 2 & 223 & 230 & 235 & 248 & 265 & 312 & 357 & 542 \\
\hline 5 & 119 & 123 & 126 & 133 & 147 & 172 & 198 & 284 \\
\hline 10 & 76 & 80 & 89 & 94 & 106 & 123 & 142 & 193 \\
\hline 20 & 48 & 50 & 52 & 54 & 72 & 82 & 107 & 130 \\
\hline 30 & 39 & 41 & 43 & 45 & 60 & 68 & 88 & 100 \\
\hline 50 & 30 & 32 & 35 & 38 & 48 & 53 & 70 & 76 \\
\hline
\end{tabular}




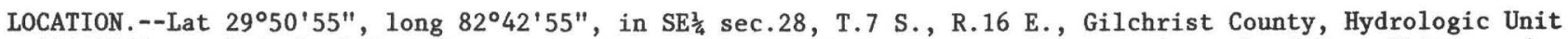
03110206 , on left bank 2.1 mi upstream from bridge on State Highway 47, $5.1 \mathrm{mi}$ south of Fort White, and $18 \mathrm{mi}$ upstream froum mouth.

DRAINAGE AREA. --1,017 $\mathrm{mi}^{2}$.

PERIOD OF RECORD ANALYZED.--April 1928 to March 1929, April 1933 to March 1978.

\begin{tabular}{|c|c|c|c|c|c|c|c|c|}
\hline \multirow{2}{*}{$\begin{array}{l}\text { Recurrence } \\
\text { interval } \\
\text { in years }\end{array}$} & MAGNITUD & $\begin{array}{l}\text { ND FR } \\
\text { Lowest } \\
\text { for }\end{array}$ & $\begin{array}{l}\text { UENCY } \\
\text { verage } \\
\text { ndicat }\end{array}$ & $\begin{array}{l}\text { ANN } \\
\text { low, } \\
\text { numl }\end{array}$ & $\begin{array}{l}\text { I LOW } \\
\text { n cubi } \\
r \text { of }\end{array}$ & $\begin{array}{l}\text { LOWS } \\
\text { feet } \\
\text { nsecut }\end{array}$ & $\begin{array}{l}\text { er sec } \\
\text { ve day }\end{array}$ & \\
\hline & 1 & 7 & 14 & 30 & 60 & 90 & 120 & 183 \\
\hline 2 & 954 & 962 & 970 & 994 & 1020 & 1070 & 1130 & 1320 \\
\hline 5 & 797 & 803 & 809 & 822 & 843 & 890 & 919 & 1030 \\
\hline 10 & 720 & 730 & 741 & 751 & 771 & 813 & 834 & 905 \\
\hline 20 & 650 & 670 & 680 & 700 & 720 & 761 & 775 & 818 \\
\hline 30 & 620 & 630 & 635 & 660 & 690 & 730 & 745 & 775 \\
\hline 50 & 590 & 600 & 610 & 620 & 670 & 710 & 717 & 732 \\
\hline
\end{tabular}

LOCATION.--Lat $29^{\circ} 48^{\prime}$, long $82^{\circ} 55^{\prime}$, in secs. 16 or 17 , T.8 S., R.14 E., Hydrologic Unit 03110205, on left

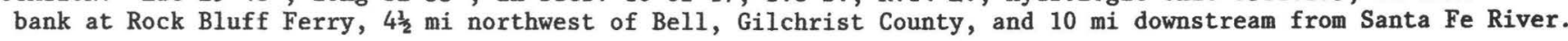
DRAINAGE AREA.--9,390 $\mathrm{mi}^{2}$, approximately, includes part of watershed in Okefenokee Swamp which is indeterminate. PERIOD OF RECORD ANALYZED.--April 1933 to March 1956.

\begin{tabular}{|c|c|c|c|c|c|c|c|c|}
\hline \multirow{3}{*}{$\begin{array}{l}\text { Recurrence } \\
\text { interval } \\
\text { in years }\end{array}$} & \multirow[t]{2}{*}{ MAGNITUD } & \multicolumn{7}{|c|}{ AND FREQUENCY OF ANNUAL LOW FLOWS } \\
\hline & & $\begin{array}{l}\text { Lowest } \\
\text { for }\end{array}$ & $\begin{array}{l}\text { average } \\
\text { indicat }\end{array}$ & $\begin{array}{l}\text { flow, } \\
\text { d numb }\end{array}$ & $\begin{array}{l}\text { n cubi } \\
r \text { of } c\end{array}$ & $\begin{array}{l}\text { feet } \\
\text { nsecut }\end{array}$ & $\begin{array}{l}\text { er sec } \\
\text { ve day }\end{array}$ & \\
\hline & 1 & 7 & 14 & 30 & 60 & 90 & $120^{\circ}$ & 183 \\
\hline 2 & 4050 & 4120 & 4170 & 4260 & 4490 & 4730 & 5000 & 5900 \\
\hline 5 & 3220 & 3300 & 3330 & 3380 & 3500 & 3630 & 3810 & 4330 \\
\hline 10 & 2890 & 2960 & 3000 & 3030 & 3110 & 3200 & 3350 & 3690 \\
\hline 20 & 2650 & 2730 & 2760 & 2790 & 2850 & 2910 & 3020 & 3240 \\
\hline 30 & 2530 & 2600 & 2640 & 2670 & 2700 & 2760 & 2860 & 3000 \\
\hline
\end{tabular}


LOCATION.--Lat $29^{\circ} 35^{\prime} 22^{\prime \prime}$, long $82^{\circ} 56^{\prime} 12^{\prime \prime}$, in NW/ sec.29, T.10 S., R.14 E., Levy County, Hydrologic Unit 03110205, on left bank about $400 \mathrm{ft}$ downstream from Fort Fannin Bridge on U.S. Highway $19,2.0 \mathrm{mi}$ southwest of Wilcox and $33 \mathrm{mi}$ upstream from mouth.

DRAINAGE AREA.--9,640 $\mathrm{mi}^{2}$, approximately, includes part of watershed in Okefenokee Swamp which is indeterminate. PERIOD OF RECORD ANALYZED.--April 1942 to March 1977.

\begin{tabular}{|c|c|c|c|c|c|c|c|c|}
\hline $\begin{array}{l}\text { Recurrence } \\
\text { interval }\end{array}$ & MAGNIT & $\begin{array}{l}\text { AND } \\
\text { Lowest } \\
\text { for }\end{array}$ & $\begin{array}{l}\text { QUENCY } \\
\text { average } \\
\text { indicat }\end{array}$ & $\begin{array}{l}\text { ANN } \\
\text { flow, } \\
\text { d numl }\end{array}$ & $\begin{array}{l}\text { LOW } \\
\text { cub } \\
\text { r of }\end{array}$ & $\begin{array}{l}\text { LOWS } \\
\text { feet } \\
\text { nsecu }\end{array}$ & $\begin{array}{l}\text { er se } \\
\text { ve da }\end{array}$ & nd, \\
\hline in years & 1 & 7 & 14 & 30 & 60 & 90 & 120 & 183 \\
\hline 2 & 5110 & 5410 & 5510 & 5650 & 5940 & 6280 & 6610 & 7530 \\
\hline 5 & 4150 & 4420 & 4510 & 4680 & 4710 & 4860 & 5040 & 5560 \\
\hline 10 & 3770 & 4020 & 4100 & 4140 & 4210 & 4290 & 4410 & 4750 \\
\hline 20 & 3500 & 3732 & 3800 & 3820 & 3850 & 3890 & 3960 & 4170 \\
\hline 30 & 3350 & 3550 & 3650 & 3680 & 3700 & 3710 & 3720 & 3790 \\
\hline 50 & 3250 & 3450 & 3500 & 3500 & 3500 & 3510 & 3530 & 3610 \\
\hline
\end{tabular}




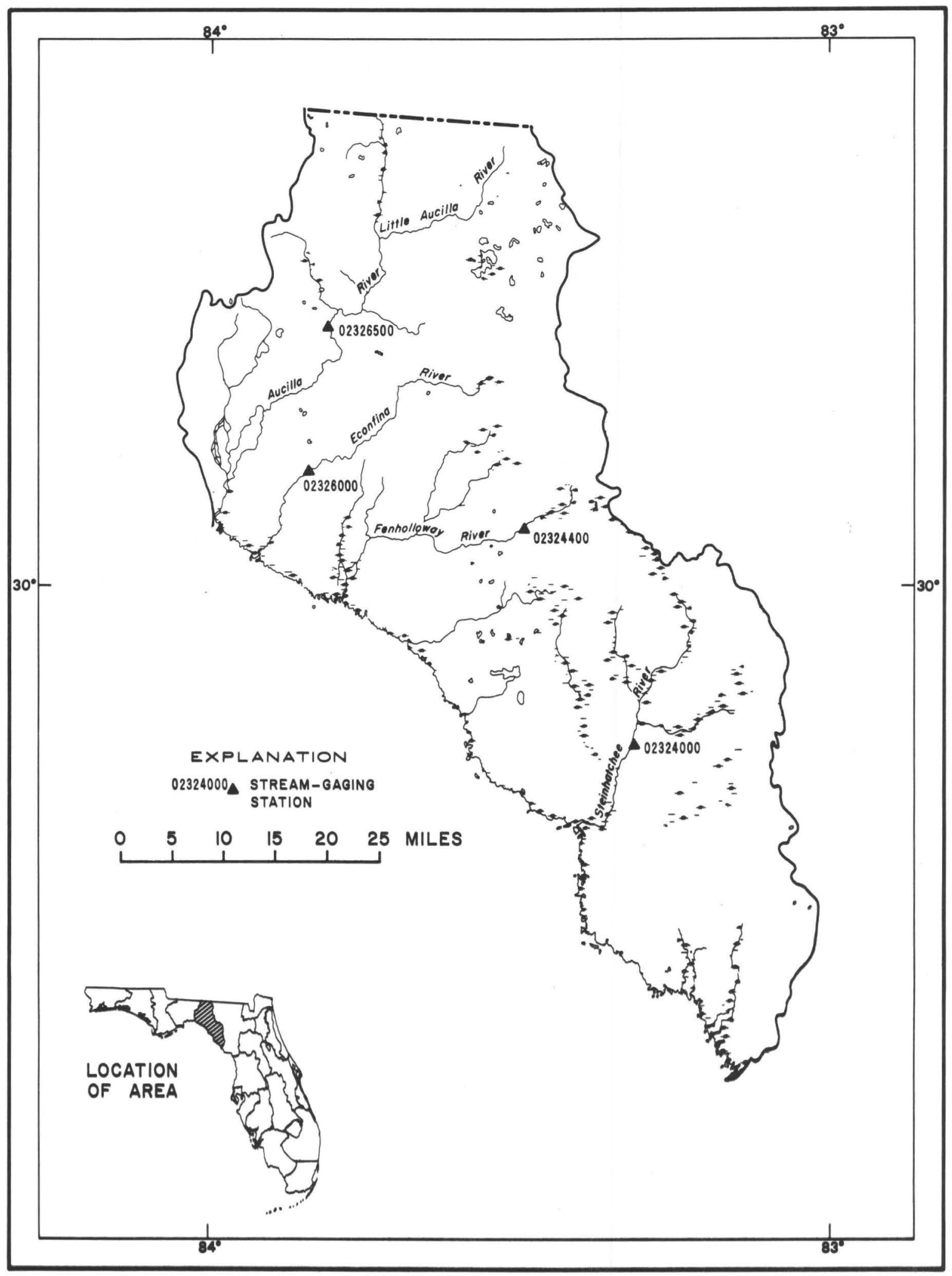

Figure 13.--Location of stream-gaging stations in the coastal area between the Suwannee and Aucilla Rivers, and the Aucilla River basin. 
LOCATION.--Lat $29^{\circ} 47^{\prime} 11^{\prime \prime}$, long $83^{\circ} 19^{\prime} 18^{\prime \prime}$, in NE $\frac{3}{4}$ sec. 16, T.8 S., R. 10 E., Taylor County, Hydrologic Unit 03110102 , on right bank $0.7 \mathrm{mi}$ downstream from Atlantic Coast Line Railroad bridge, $0.7 \mathrm{mi}$ south of Clara, 13 mi upstream from mouth, and 16 mi northwest of Cross City.

DRAINAGE AREA.--350 $\mathrm{mi}^{2}$, approximately.

PERIOD OF RECORD ANALYZED.--April 1950 to March 1978.

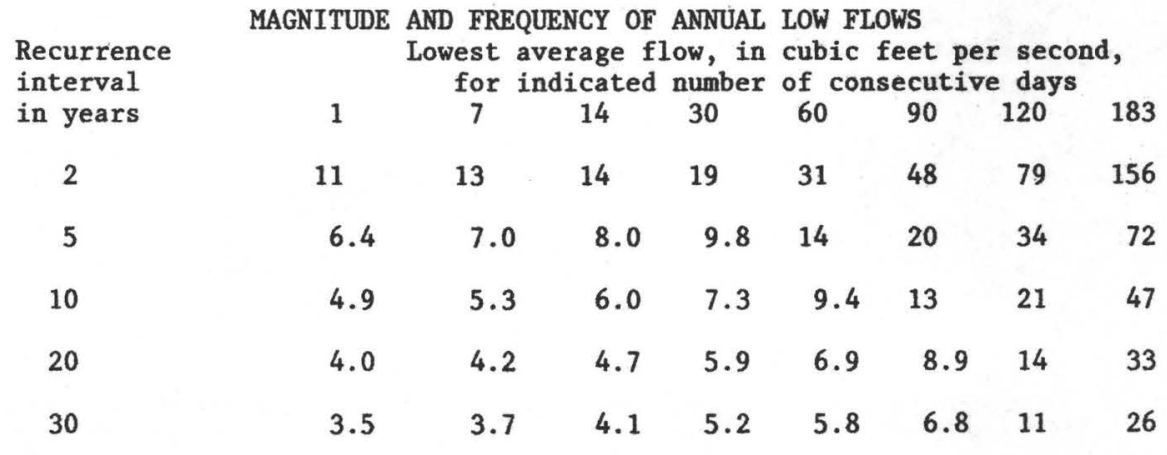

02324400 Fenholloway River near Foley, Fla.

LOCATION.--Lat $30^{\circ} 05^{\prime} 53^{\prime \prime}$, long $83^{\circ} 28^{\prime} 19^{\prime \prime}$, in NE光 sec.36, T.4 S., R.8 E., Taylor County, Hydrologic Unit 03110102 , near left bank at downstream side of bridge on U.S. Highway $27,1.8$ mi upstream from small tributary, 4 mi northeast of Foley, and 32 mi upstream from mouth.

DRAINAGE AREA. $--60 \mathrm{mi}^{2}$, approximately.

PERIOD OF RECORD ANALYZED.--April 1956 to March 1977.

\begin{tabular}{|c|c|c|c|c|c|c|c|c|}
\hline $\begin{array}{l}\text { Recurrence } \\
\text { interval }\end{array}$ & MAGNITUDE & $\begin{array}{l}\text { D FREQ } \\
\text { west a } \\
\text { for } i\end{array}$ & $\begin{array}{l}\text { CY of } \\
\text { age } f 1 \\
\text { cated }\end{array}$ & $\begin{array}{l}\text { ANNUAL } \\
\text { OW, in } \\
\text { number }\end{array}$ & $\begin{array}{l}\text { LOW FL } \\
\text { cubic } \\
\text { of cons }\end{array}$ & $\begin{array}{l}\text { WS } \\
\text { eet pe } \\
\text { ecutiv }\end{array}$ & $\begin{array}{l}r \text { secor } \\
\text { e days }\end{array}$ & \\
\hline in years & 1 & 7 & 14 & 30 & 60 & 90 & 120 & 183 \\
\hline 2 & 1.8 & 2.0 & 2.1 & 2.6 & 4.0 & 6.7 & 9.9 & 19 \\
\hline 5 & 1.2 & 1.2 & 1.3 & 1.4 & 1.8 & 2.4 & 3.5 & 6.4 \\
\hline 10 & 1.0 & 1.0 & 1.1 & 1.1 & 1.2 & 1.5 & 2.0 & 3.3 \\
\hline 20 & .80 & .84 & .85 & .88 & .90 & 1.0 & 1.2 & 1.9 \\
\hline 30 & .72 & .74 & .75 & .76 & .80 & .85 & .9 & 1.0 \\
\hline
\end{tabular}


COASTAL AREA BETWEEN SUWANNEE RIVER AND AUCILLA RIVERS

02326000 Econfina River near Perry, Fla.

LOCATION.--Lat $30^{\circ} 10^{\prime} 14^{\prime \prime}$, long $83^{\circ} 49^{\prime} 26^{\prime \prime}$, in NE光 sec.4, T.4 S., R.5 E., Taylor County, Hydrologic Unit 03110102 , on right bank $10 \mathrm{ft}$ downstream from highway bridge, $3.0 \mathrm{mi}$ downstream from Natural Well Branch, $3.9 \mathrm{mi}$ upstream from bridge on U.S. Highway $98,14 \mathrm{mi}$ upstream from mouth, and 14.7 mi northwest of Perry.

DRAINAGE AREA. - - $198 \mathrm{mi}^{2}$.

PERIOD OF RECORD ANALYZED.--April 1947 to March 1977.

Recurrence interval in years

2

5

10

20

30
MAGNITUDE AND FREQUENCY OF ANNUAL LOW FLOWS

Lowest average flow, in cubic feet per second, for indicated number of consecutive days

$\begin{array}{llllllll}1 & 7 & 14 & 30 & 60 & 90 & 120 & 183\end{array}$

20

20

21

$22 \quad 25$

32

38

54

11

11

11

$12 \quad 13$

15

17

24

$$
7.3
$$

7.6

7.8

8.4

9.210

12

16

5.1

5.3

5.7

6.2

6.8

7.5

$8.5 \quad 11$

4.0

4.4

4.7

5.2

5.8

6.3

7.19 .5 


\section{AUCILLA RIVER BASIN}

02326500 Aucilla River at Lamont, Fla.

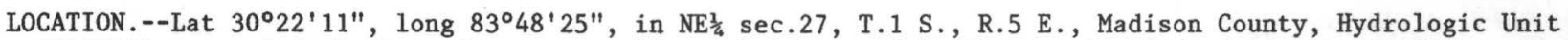
03110103, near left bank on downstream side of bridge on U.S. Highway 19, 0.6 mi southeast of Lamont, and 34 mi upstream from mouth.

DRAINAGE AREA. $--747 \mathrm{mi}^{2}$.

PERIOD OF RECORD ANALYZED.--April 1950 to March 1978.

REMARKS.--Pumpage above and below station for irrigation during dry seasons.

Recurrence interval

in years

2

5

10

20

30

MAGNITUDE AND FREQUENCY OF ANNUAL LOW FLOWS

Lowest average flow, in cubic feet per second, for indicated number of consecutive days

$\begin{array}{llllllll}1 & 7 & 14 & 30 & 60 & 90 & 120 & 183\end{array}$

14

15

17

19

24

33

$42 \quad 65$

1.6

1.8

2.0

3.0

4.0

5.6

7.6

12

0

0

0

$$
.10
$$

1.3

1.9

2.6

\section{0}

0

0

0

.42

.68

1.0

1.7

0

0

0

$.20 \quad .50 \quad .70$

1.1 


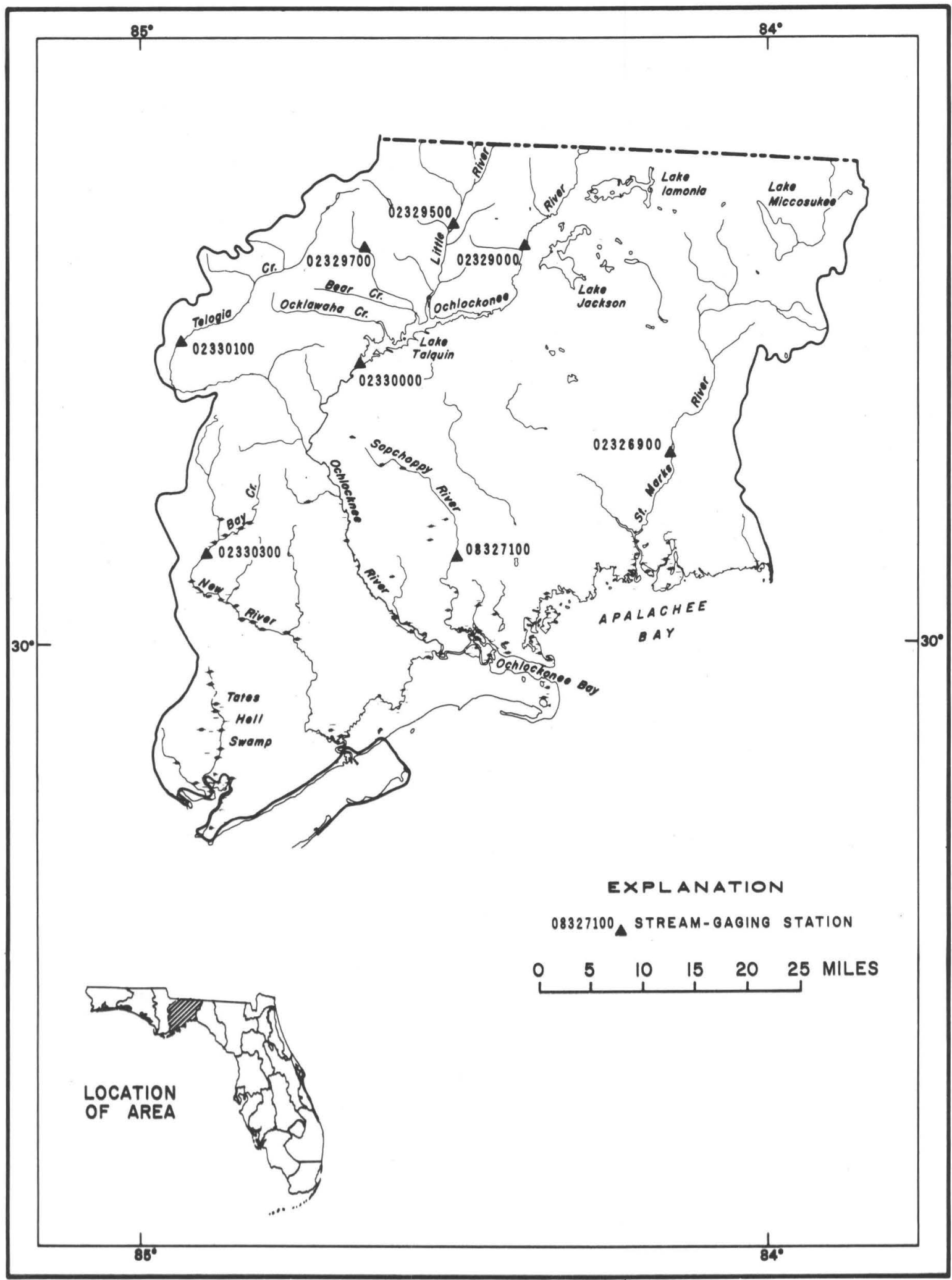

Figure 14.--Location of stream-gaging stations in the St. Marks and Ochlockonee River basins and the coastal area between the 0chlockonee and Apalachicola Rivers. 
ST. MARKS RIVER BASIN

02326900 St. Marks River near Newport, F1a.

LOCATION.--Lat $30^{\circ} 16^{\prime} 00^{\prime \prime}$, long $84^{\circ} 09^{\prime} 00^{\prime \prime}$, in SE $\frac{1}{4} \mathrm{sec} .32$, T.2 S., R.2 E., Wakulla County, Hydrologic Unit 03120001 , on left bank 0.9 mi downstream from Rhodes Springs, 6 mi north of Newport, 11 mi upstream from Wakulla River, and $14 \mathrm{mi}$ upstream from mouth.

DRAINAGE AREA.--535 $\mathrm{mi}^{2}$ includes drainage area for Lake Miccosukee, $290 \mathrm{mi}^{2}$, which contributes to St. Marks River at high stages.

PERIOD OF RECORD ANALYZED.--April 1950 to March 1978.

Recurrence

interval

in years

2

5

10

20

30

MAGNITUDE AND FREQUENCY OF ANNUAL LOW FLOWS

Lowest average flow, in cubic feet per second, for indicated number of consecutive days

$\begin{array}{llllllll}1 & 7 & 14 & 30 & 60 & 90 & 120 & 183\end{array}$

$\begin{array}{llllllll}404 & 411 & 419 & 441 & 462 & 490 & 507 & 559\end{array}$

$\begin{array}{llllllll}346 & 354 & 363 & 378 & 391 & 409 & 422 & 457\end{array}$

$\begin{array}{llllllll}323 & 332 & 342 & 353 & 362 & 374 & 388 & 415\end{array}$

$\begin{array}{llllllll}308 & 318 & 329 & 335 & 341 & 349 & 364 & 385\end{array}$

$\begin{array}{llllllll}300 & 311 & 322 & 326 & 330 & 335 & 352 & 360\end{array}$


LOCATION.--Lat $30^{\circ} 07^{\prime} 4^{\prime \prime}$, long $84^{\circ} 29^{\prime} 40^{\prime \prime}$ in NW/ sec.24, T.4 S., R.3 W., Wakulla County, Hydrologic Unit

03120003 , Apalachicola National Forest, near left bank on downstream side of bridge on U.S. Forest Road 346A, $4.7 \mathrm{mi}$ north of Sopchoppy, $5.2 \mathrm{mi}$ upstream from Duval Branch, and $24 \mathrm{mi}$ upstream from mouth.

DRAINAGE AREA. $--102 \mathrm{mi}^{2}$.

PERIOD OF RECORD ANALYZED.--April 1965 to March 1978.

\begin{tabular}{|c|c|c|c|c|c|c|c|c|}
\hline \multirow{2}{*}{$\begin{array}{l}\text { Recurrence } \\
\text { interval } \\
\text { in years }\end{array}$} & MAGNITUDE & $\begin{array}{l}\text { FRE } \\
\text { west } \\
\text { for }\end{array}$ & $\begin{array}{l}\text { QUENCY OF } \\
\text { average } f \\
\text { indicated }\end{array}$ & $\begin{array}{l}\text { ANNUAL } \\
\text { Low, in } \\
\text { number }\end{array}$ & $\begin{array}{l}\text { LOW FI } \\
\text { cubic } \\
\text { of cor }\end{array}$ & $\begin{array}{l}\text { oWS } \\
\text { feet pe } \\
\text { secutiv }\end{array}$ & $\begin{array}{l}\text { r seco } \\
\text { e days }\end{array}$ & \\
\hline & 1 & 7 & 14 & 30 & 60 & 90 & $120^{\circ}$ & 183 \\
\hline 2 & 2.0 & 2.3 & 2.6 & 3.2 & 7.1 & 21 & 51 & 105 \\
\hline 5 & 1.4 & 1.6 & 1.7 & 2.2 & 3.4 & 7.8 & 22 & 60 \\
\hline 10 & 1.1 & 1.3 & 1.5 & 1.8 & 2.6 & 4.8 & 13 & 44 \\
\hline 20 & 1.0 & 1.1 & 1.3 & 1.7 & 2.1 & 3.2 & 8.3 & 32 \\
\hline
\end{tabular}

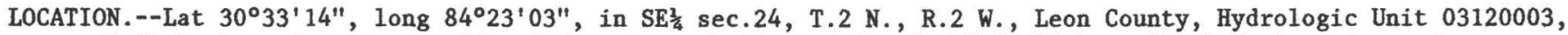
near left bank on downstream side of downstream bridge on divided U.S. Highway $27,0.8$ mi upstream from Seaboard Air Line Railroad bridge, $4.0 \mathrm{mi}$ downstream from Mill Creek, $5.0 \mathrm{mi}$ southeast of Havana, and $94 \mathrm{mi}$ upstream from mouth.

DRAINAGE AREA. --1,140 $\mathrm{mi}^{2}$, approximately. At site used prior to January 1929, 1,220 mi², approximately.

PERIOD OF RECORD ANALYZED.--April 1927 to March 1978.

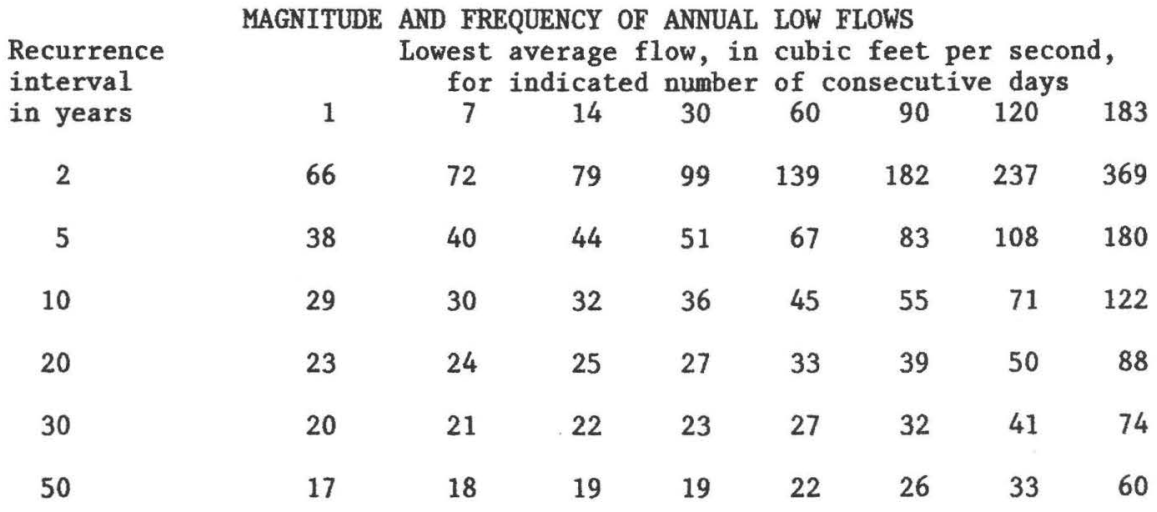


LOCATION.--Lat $30^{\circ} 35^{\prime} 14^{\prime \prime}$, long $84^{\circ} 29^{\prime} 48^{\prime \prime}$, in NW 03120003, near right bank at downstream side of bridge on State Highway 12, 0.5 mi southwest of Shady Rest, $1.1 \mathrm{mi}$ downstream from confluence of Willacoochee and Attapulgus Creeks, $4.5 \mathrm{mi}$ east of Quincy, and $12 \mathrm{mi}$ upstream from mouth.

DRAINAGE AREA. - - $237 \mathrm{mi}^{2}$.

PERIOD OF RECORD ANALYZED.--April 1950 to March 1968.

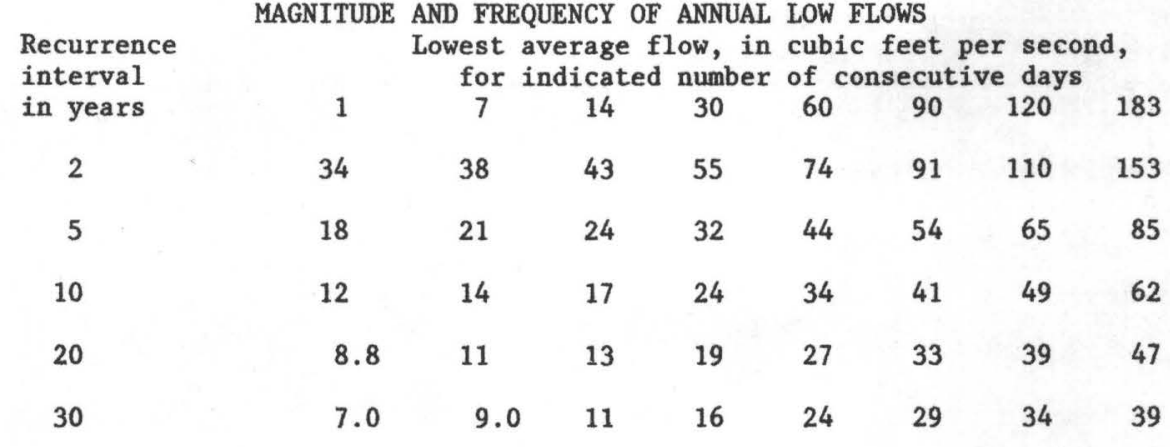

02329700 Rocky Comfort Creek near Quincy, Fla.

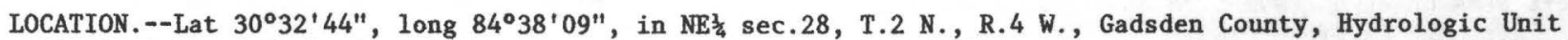
03120003, on left bank $15 \mathrm{ft}$ upstream from bridge on State Highway 274, $1.3 \mathrm{mi}$ upstream from Vote Creek, $4.5 \mathrm{mi}$ southwest of Quincy, and $9.2 \mathrm{mi}$ upstream from mouth.
\end{abstract}

DRAINAGE AREA. $--9.46 \mathrm{mi}^{2}$.

PERIOD OF RECORD ANALYZED.--April 1965 to March 1978.

REMARKS.--Data adjusted by use of a flow correlation analysis with gaging station 02329500, Little River near Quincy.

Recurrence
interval
in years
2
5
10
20

MAGNITUDE AND FREQUENCY OF ANNUAL LOW FLOWS

Lowest average flow, in cubic feet per second, for indicated number of consecutive days

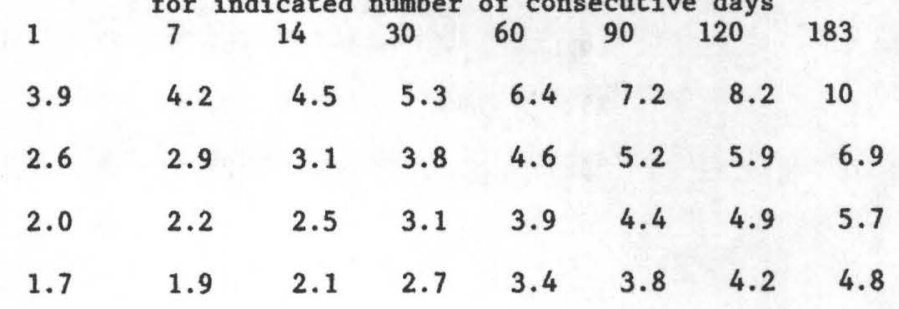


LOCATION.--Lat $30^{\circ} 25^{\prime} 35^{\prime \prime}$, long $84^{\circ} 55^{\prime} 40^{\prime \prime}$, in NW/ sec.3, T.1 S., R.7 W., Liberty County, Hydrologic Unit 03120003 , near left bank at downstream side of bridge on State Highway 20,600 ft upstream from White Branch, $3.0 \mathrm{mi}$ east of Bristol, and $33 \mathrm{mi}$ upstream from mouth.

DRAINAGE AREA. $--126 \mathrm{mi}^{2}$.

PERIOD OF RECORD ANALYZED.--April 1950 to March 1971, April 1975 to March 1978.

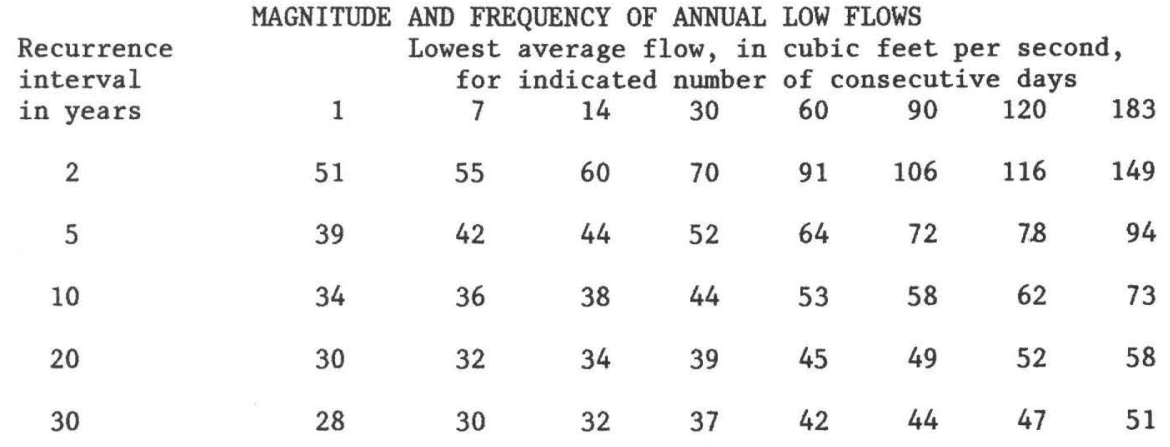


LOCATION.--Lat $30^{\circ} 07^{\prime} 40^{\prime \prime}$, long $84^{\circ} 53^{\prime} 45^{\prime \prime}$, in SW $\frac{1}{4}$ sec.13, T.4 S., R.7 W., Liberty County, Hydrologic Unit 03130013, Apalachicola National Forest, near center on downstream side of Carr Bridge on U.S. Forest Road 13, $2.2 \mathrm{mi}$ upstream from West Prong New River, $4.5 \mathrm{mi}$ southeast of Wilma, and $40 \mathrm{mi}$ upstream from mouth.

DRAINAGE AREA. $--81.7 \mathrm{mi}^{2}$.

PERIOD OF RECORD ANALYZED.--April 1965 to March 1978.

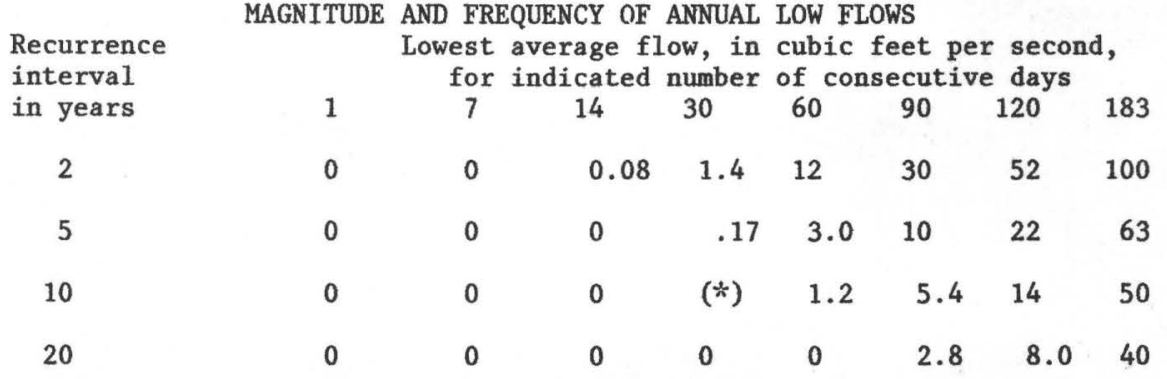

* Less than $0.05 \mathrm{ft}^{3} / \mathrm{s}$. 


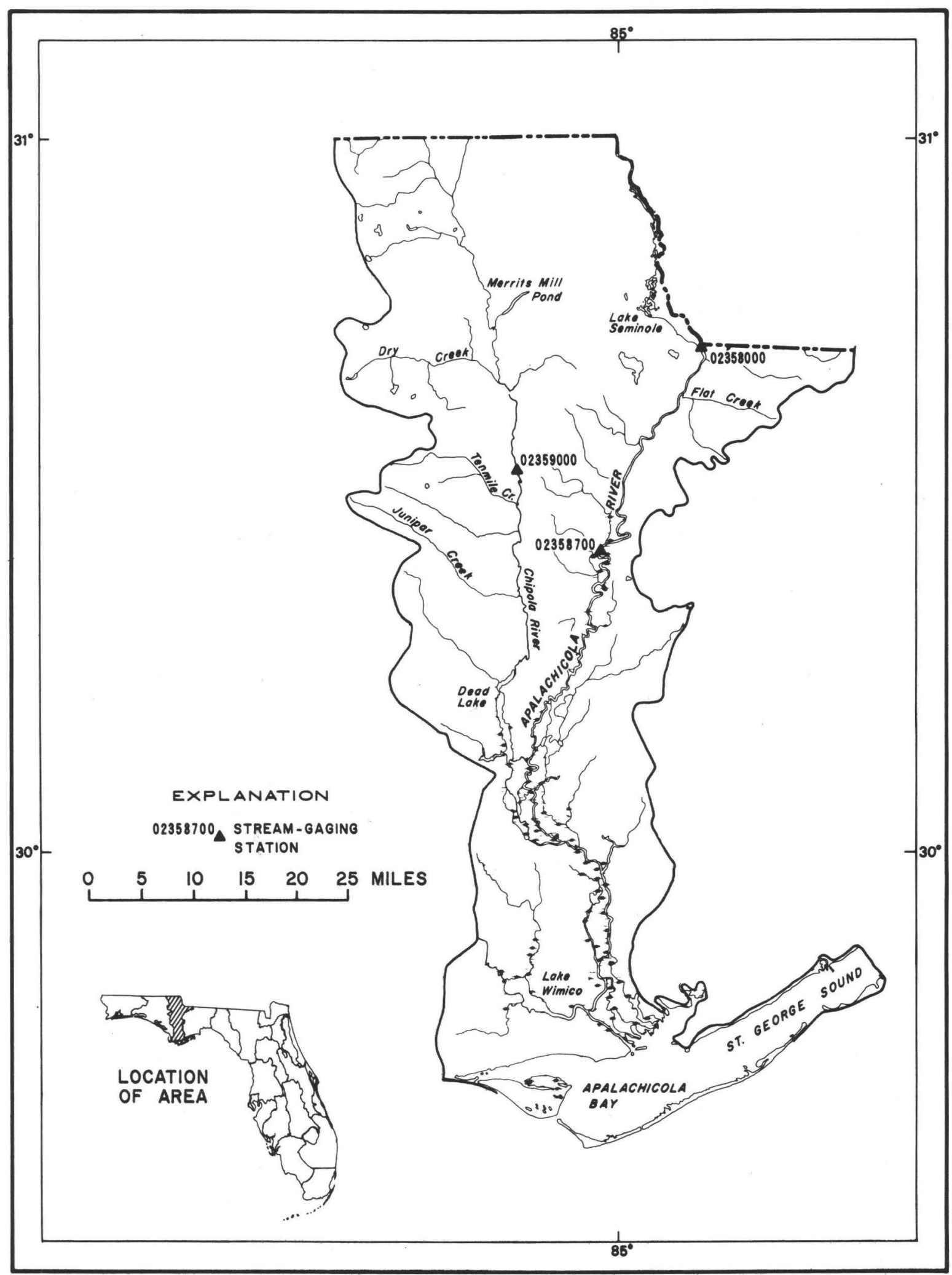

Figure 15.--Location of stream-gaging stations in the Apalachicola River basin including the Chipola River basin. 
LOCATION.--Lat $30^{\circ} 42^{\prime} 03^{\prime \prime}$, long $84^{\circ} 51^{\prime} 33^{\prime \prime}$, in NW/ sec.32, T.4 N., R.6 W., Jackson County, Hydrologic Unit

03130011, on downstream side of right main pier on U.S. Highway 90, 0.6 mi downstream from Jim Woodruff Dam, $0.6 \mathrm{mi}$ upstream from Mosquito Creek, $1.0 \mathrm{mi}$ west of Chattahoochee, and $106 \mathrm{mi}$ upstream from mouth.

DRAINAGE AREA. --17,200 $\mathrm{mi}^{2}$, approximately.

PERIOD OF RECORD ANALYZED.--April 1957 to March 1978.

REMARKS.--Flow regulated by Lake Seminole Reservoir (02357500) 0.6 mi upstream since Feb. 4, 1957. Walter F. George Reservoir (02343240) since 1962, Bartlett's Ferry Reservoir (02341000) since 1926, and Lake Sidney Lanier Reservoir (02324400) since 1956.

\begin{tabular}{|c|c|c|c|c|c|c|c|c|}
\hline $\begin{array}{l}\text { Recurrence } \\
\text { interval }\end{array}$ & & $\begin{array}{l}\text { Lowes } \\
\text { fo }\end{array}$ & $\begin{array}{l}\text { average } \\
\text { indicat }\end{array}$ & flow, & in of & $\begin{array}{l}\text { c feet } \\
\text { onsecu }\end{array}$ & $\begin{array}{l}\text { per se } \\
\text { ive day }\end{array}$ & nd, \\
\hline in years & 1 & 7 & 14 & 30 & 60 & 90 & 120 & 183 \\
\hline 2 & 9360 & 9700 & 9930 & 10300 & 10700 & 11400 & 12200 & 13600 \\
\hline 5 & 7200 & 8000 & 8200 & 8540 & 8700 & 9490 & 10200 & $11 \lesssim 00$ \\
\hline 10 & 6200 & 7000 & 7300 & 7730 & 8000 & 9000 & 9440 & 10800 \\
\hline 20 & 5500 & 6300 & 6600 & 7100 & 7400 & 8500 & 8950 & 10300 \\
\hline 30 & 5200 & 5900 & 6200 & 6500 & 7000 & 8100 & 8600 & 9700 \\
\hline
\end{tabular}

02358700 Apalachicola River near Blountstown, Fla.

LOCATION.--Lat $30^{\circ} 25^{\prime} 30^{\prime \prime}$, long $85^{\circ} 01^{\prime} 53^{\prime \prime}$, in NE $\frac{3}{4}$ sec.3, T.1 S., R. 8 W., Calhoun County, Hydrologic Unit 03130011 , on right bank $500 \mathrm{ft}$ upstream from Neal Lumber Company Landing at McNeal, $0.5 \mathrm{mi}$ upstream from old River cutoff, $1.5 \mathrm{mi}$ southeast of Blountstown, and $78 \mathrm{mi}$ upstream from mouth.

DRAINAGE AREA. $--17,600 \mathrm{mi}^{2}$, approximately.

PERIOD OF RECORD ANALYZED.--April 1958 to March 1977.

\begin{tabular}{|c|c|c|c|c|c|c|c|c|}
\hline $\begin{array}{l}\text { Recurrence } \\
\text { interval }\end{array}$ & MAGNITU & $\begin{array}{l}\text { AND FR } \\
\text { Lowest } \\
\text { for }\end{array}$ & $\begin{array}{l}\text { QUENCY } \\
\text { average } \\
\text { indicat }\end{array}$ & $\begin{array}{l}\text { OF ANN } \\
\text { flow, } \\
\text { ed numl }\end{array}$ & $\begin{array}{l}\text { AL LOW } \\
\text { in cub } \\
\text { er of }\end{array}$ & $\begin{array}{l}\text { FLOWS } \\
\text { c feet } \\
\text { onsecu }\end{array}$ & $\begin{array}{l}\text { per sec } \\
\text { ive day }\end{array}$ & $\begin{array}{l}\text { ond, } \\
\text { s }\end{array}$ \\
\hline in years & 1 & 7 & 14 & 30 & 60 & 90 & $120^{\circ}$ & 183 \\
\hline 2 & 9980 & 10410 & 10670 & 11040 & 11530 & 12320 & 13180 & 14690 \\
\hline 5 & 8100 & 8540 & 8780 & 9150 & 9530 & 10090 & 10790 & 12260 \\
\hline 10 & 7170 & 7660 & 7910 & 8290 & 8730 & 9240 & 9800 & 11290 \\
\hline 20 & 6460 & 6980 & 7260 & 7640 & 8180 & 8670 & 9080 & 10610 \\
\hline
\end{tabular}


LOCATION.--Lat $30^{\circ} 32^{\prime} 02^{\prime \prime}$, long $85^{\circ} 09^{\prime} 55^{\prime \prime}$, in NW/ sec.32, T.2 N., R.9 W., Calhoun County, Hydrologic Unit 03130012 , on right bank on downstream side of bridge on State Highway 274, 0.9 mi downstream from Holliman Branch, $3.5 \mathrm{mi}$ southwest of Altha, and 54 mi upstream from mouth.

DRAINAGE AREA. $--781 \mathrm{mi}^{2}$.

REVISED RECORDS.--WSP 1384: Drainage area. WSP 1504: 1924, 1925 (m), 1926.

PERIOD OF RECORD ANALYZED.--April 1922 to March 1927, April 1930 to March 1931, April 1943 to March 1978.

Recurrence

interval

in years

2

5

10

20

30

50
MAGNITUDE AND FREQUENCY OF ANNUAL LOW FLOWS

Lowest average flow, in cubic feet per second, for indicated number of consecutive days

$\begin{array}{lllllll}7 & 14 & 30 & 60 & 90 & 120 & 183\end{array}$

$\begin{array}{lllllll}636 & 653 & 688 & 741 & 789 & 841 & 952\end{array}$

$\begin{array}{lllllll}491 & 506 & 536 & 571 & 597 & 629 & 702\end{array}$

$\begin{array}{llllllll}461 & 491 & 506 & 536 & 571 & 597 & 629 & 702\end{array}$

$\begin{array}{llllllll}401 & 427 & 441 & 470 & 500 & 520 & 544 & 606\end{array}$

$\begin{array}{llllllll}357 & 379 & 393 & 422 & 448 & 466 & 484 & 539\end{array}$

$\begin{array}{llllllll}335 & 355 & 369 & 400 & 422 & 440 & 455 & 507\end{array}$

$\begin{array}{llllllll}312 & 330 & 344 & 374 & 397 & 413 & 426 & 475\end{array}$




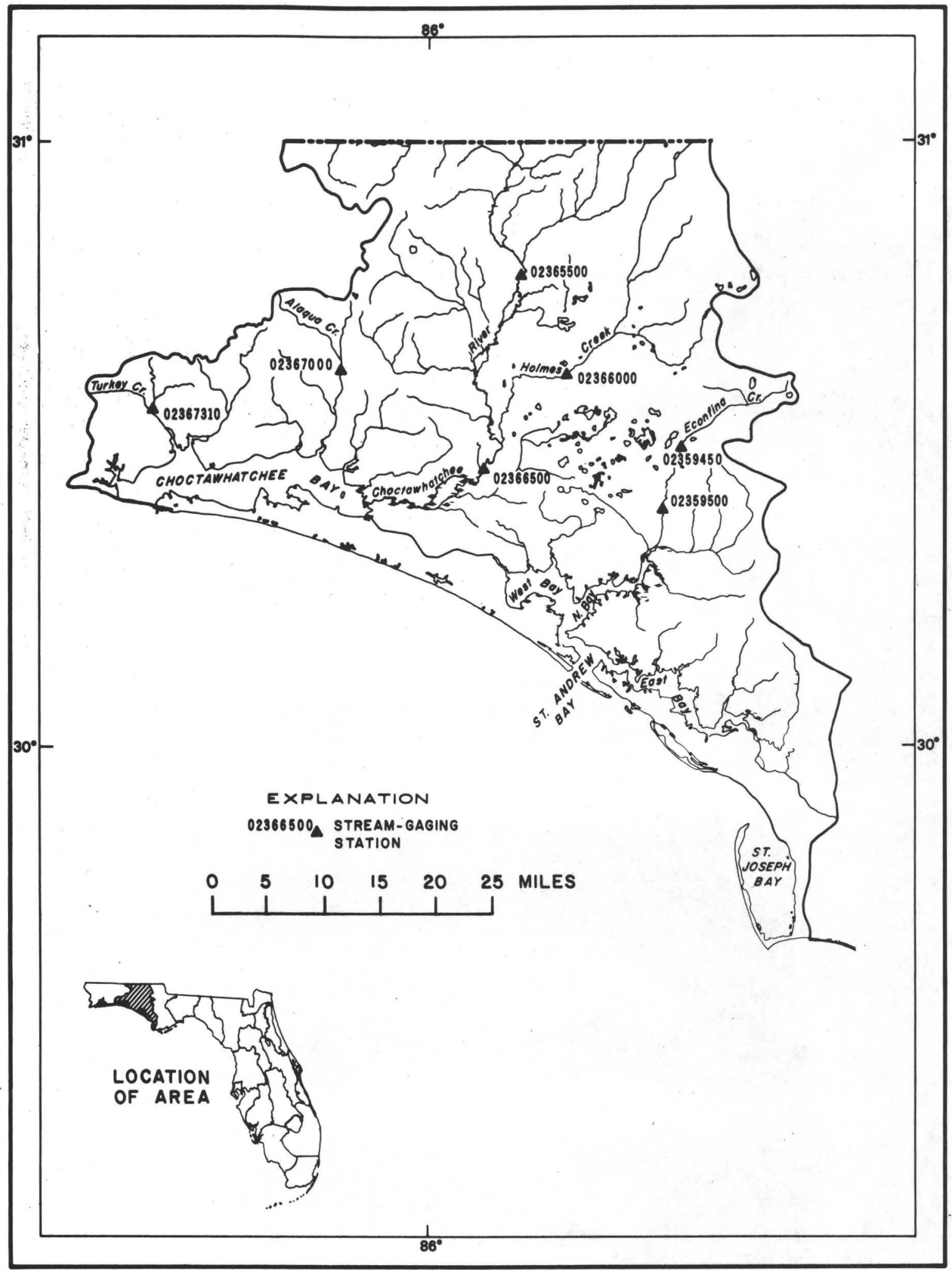

Figure 16.--Location of stream-gaging stations in Choctawhatchee River basin below Pea River, and inflow areas for St. Andrew and Choctawhatchee Bays. 
ST. ANDREW BAY, INFLOW AREA

02359450 Econfina Creek near Fountain, Fla.

LOCATION.--Lat $30^{\circ} 28^{\prime} 55^{\prime \prime}$, long $85^{\circ} 31^{\prime} 30^{\prime \prime}$, in SE光 sec.15, T.1 N., R.13 W., Washington County, Hydrologic Unit 03140101, near left bank $15 \mathrm{ft}$ downstream from Walsingham Bridge on county road, $0.2 \mathrm{mi}$ upstream from Mitchel1 Mill Creek, $6.0 \mathrm{mi}$ west of Fountain, and $23 \mathrm{mi}$ upstream from mouth.

DRAINAGE AREA. $--70.2 \mathrm{mi}^{2}$.

PERIOD OF RECORD ANALYZED.--April 1965 to March 1978.

REMARKS.--Data adjusted by use of a flow correlation analysis with gaging station 02359500, Econfina Creek near Bennett.

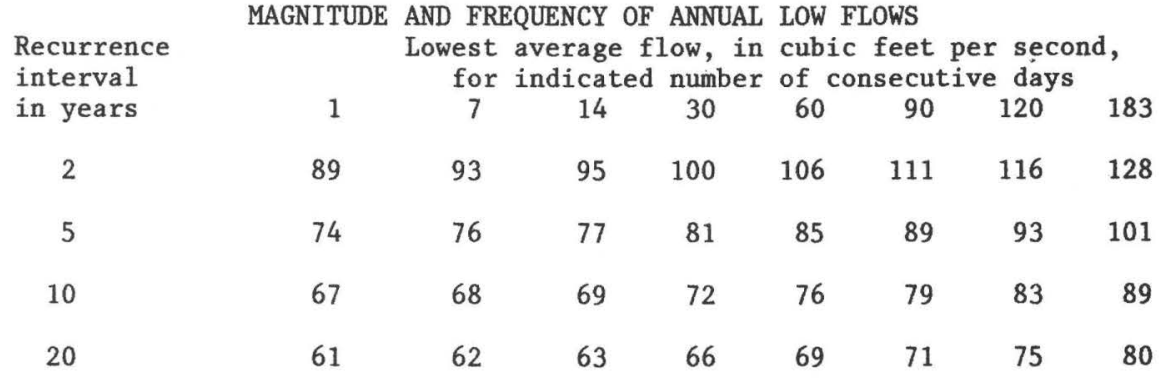

02359500 Econfina Creek near Bennett, Fla.

LOCATION.--Lat $30^{\circ} 23^{\prime} 04^{\prime \prime}$, long $85^{\circ} 33^{\prime} 24^{\prime \prime}$, in SE $\frac{1}{4}$ sec.20, T.1 S., R.13 W., Bay County, Hydrologic Unit 03140101, near center of span on downstream side of bridge on State Highway $388,0.5$ mi downstream from 0ld Mill Branch, $1.6 \mathrm{mi}$ southwest of Bennett, and $11 \mathrm{mi}$ upstream from mouth.

DRAINAGE AREA. --122 $\mathrm{mi}^{2}$.

PERIOD OF RECORD ANALYZED.--April 1936 to March 1978.

Recurrence interval

in years

2

5

10

20

30

50

MAGNITUDE AND FREQUENCY OF ANNUAL LOW FLOWS

Lowest average flow, in cubic feet per second, for indicated number of consecutive days

$\begin{array}{lllllll}7 & 14 & 30 & 60 & 90 & 120 & 183\end{array}$

$\begin{array}{llllllll}402 & 412 & 418 & 429 & 443 & 455 & 466 & 491\end{array}$

$\begin{array}{llllllll}362 & 368 & 372 & 381 & 393 & 402 & 412 & 432\end{array}$

$\begin{array}{llllllll}342 & 346 & 350 & 358 & 368 & 376 & 386 & 402\end{array}$

$\begin{array}{llllllll}326 & 329 & 332 & 340 & 348 & 356 & 365 & 378\end{array}$

$\begin{array}{llllllll}317 & 320 & 323 & 330 & 336 & 345 & 355 & 365\end{array}$

$\begin{array}{llllllll}309 & 311 & 313 & 320 & 326 & 335 & 344 & 352\end{array}$




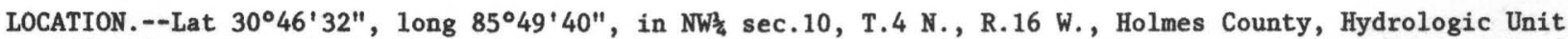
03140203, near right bank on downstream side of bridge on U.S. Highway 90,300 ft downstream from Louisville and Nashville Railroad bridge, $0.8 \mathrm{mi}$ west of Caryville, $1.8 \mathrm{mi}$ downstream from Wrights Creek, and $64 \mathrm{mi}$ upstream from mouth.

DRAINAGE AREA. $--3,499 \mathrm{mi}^{2}$.

PERIOD OF RECORD ANALYZED.--April 1930 to March 1978.

\begin{tabular}{|c|c|c|c|c|c|c|c|c|}
\hline \multirow{2}{*}{$\begin{array}{l}\text { Recurrence } \\
\text { interval } \\
\text { in years }\end{array}$} & MAGNITUD & $\begin{array}{l}\text { AND FF } \\
\text { Lowest } \\
\text { for }\end{array}$ & $\begin{array}{l}\text { UENCY } \\
\text { verage } \\
\text { ndicat }\end{array}$ & $\begin{array}{l}\text { F ANN } \\
\text { flow, } \\
\text { d numl }\end{array}$ & $\begin{array}{l}\text { L LOW } \\
\text { n cub } \\
\text { r of }\end{array}$ & $\begin{array}{l}\text { LOWS } \\
\text { feet } \\
\text { nsecut }\end{array}$ & $\begin{array}{l}\text { per se } \\
\text { ive da }\end{array}$ & nd, \\
\hline & 1 & 7 & 14 & 30 & 60 & 90 & 120 & 183 \\
\hline 2 & 1210 & 1280 & 1350 & 1520 & 1794 & 2110 & 2350 & 2820 \\
\hline 5 & 939 & 985 & 1040 & 1160 & 1330 & 1520 & 1670 & 2010 \\
\hline 10 & 837 & 874 & 921 & 1020 & 1150 & 1300 & 1410 & 1700 \\
\hline 20 & 767 & 799 & 845 & 925 & 1030 & 1140 & 1240 & 1490 \\
\hline 30 & 730 & 765 & 810 & 880 & 980 & 1050 & 1150 & 1400 \\
\hline 50 & 702 & 729 & 775 & 839 & 925 & 993 & 1070 & 1300 \\
\hline
\end{tabular}

02366000 Holmes Creek at Vernon, Fla.

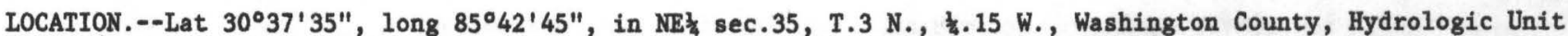
03140203 , near left bank on downstream side of bridge on State Highway 79 at Vernon, 0.2 mi downstream from Pippin Mill Creek, and 25 mi upstream from mouth.

DRAINAGE AREA. $--386 \mathrm{mi}^{2}$.

PERIOD OF RECORD ANALYZED.--April 1950 to March 1978.

Recurrence
interval
in years
2
5
10
20
30

MAGNITUDE AND FREQUENCY OF ANNUAL LOW FLOWS

Lowest average flow, in cubic feet per second, for indicated number of consecutive days

$\begin{array}{llllllll}1 & 7 & 14 & 30 & 60 & 90 & 120 & 183\end{array}$

$\begin{array}{llllllll}310 & 315 & 319 & 328 & 335 & 351 & 371 & 413\end{array}$

$\begin{array}{llllllll}280 & 284 & 286 & 290 & 297 & 303 & 310 & 333\end{array}$

$\begin{array}{llllllll}265 & 269 & 271 & 275 & 285 & 288 & 291 & 307\end{array}$

$\begin{array}{llllllll}253 & 257 & 260 & 265 & 279 & 279 & 281 & 290\end{array}$

$\begin{array}{llllllll}245 & 250 & 254 & 260 & 276 & 276 & 278 & 282\end{array}$ 


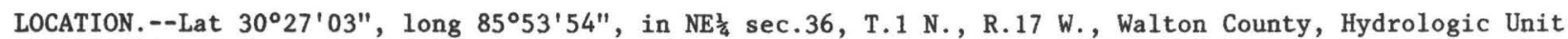
03140203, on downstream fender pile at center swing pier of bridge on State Highway $20,4.0$ mi southeast of Bruce, $5.8 \mathrm{mi}$ downstream from Holmes Creek, and $21 \mathrm{mi}$ upstream from mouth.

DRAINAGE AREA. $--4,384 \mathrm{mi}^{2}$.

PERIOD OF RECORD ANALYZED.--April 1931 to March 1978.

\begin{tabular}{|c|c|c|c|c|c|c|c|c|}
\hline $\begin{array}{l}\text { Recurrence } \\
\text { interval }\end{array}$ & 28082 & $\begin{array}{r}\text { Lowest } \\
\text { for }\end{array}$ & $\begin{array}{l}\text { average } \\
\text { indicat }\end{array}$ & $\begin{array}{l}\text { F ANN } \\
\text { flow, } \\
\text { d numb }\end{array}$ & $\begin{array}{l}\text { LOW } \\
\text { cubi }\end{array}$ & $\begin{array}{l}\text { feet } \\
\text { nsecut }\end{array}$ & $\begin{array}{l}\text { er sec } \\
\text { ve day }\end{array}$ & nd, \\
\hline in years & 1 & 7 & 14 & 30 & 60 & 90 & 120 & 183 \\
\hline 2 & 2120 & 2180 & 2280 & 2470 & 2800 & 3190 & 3490 & 4050 \\
\hline 5 & 1750 & 1780 & 1840 & 1960 & 2170 & 2400 & 2580 & 2960 \\
\hline 10 & 1600 & 1630 & 1670 & 1770 & 1930 & 2090 & 2230 & 2540 \\
\hline 20 & 1500 & 1530 & 1560 & 1630 & 1760 & 1870 & 1980 & 2250 \\
\hline 30 & 1440 & 1470 & 1500 & 1570 & 1670 & 1760 & 1850 & 2100 \\
\hline 50 & 1410 & 1440 & 1450 & 1500 & 1600 & 1660 & 1750 & 1980 \\
\hline
\end{tabular}




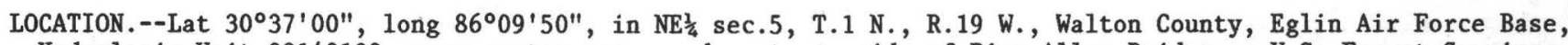
Hydrologic Unit 03140102 , near center span on downstream side of Pine Allen Bridge on U.S. Forest Service road 200, $0.8 \mathrm{mi}$ upstream from Davis Branch, $8.0 \mathrm{mi}$ southwest of DeFuniak Springs, and $11 \mathrm{mi}$ upstream from mouth.

DRAINAGE AREA. $--65.6 \mathrm{mi}^{2}$.

PERIOD OF RECORD ANALYZED.--April 1952 to March 1978.

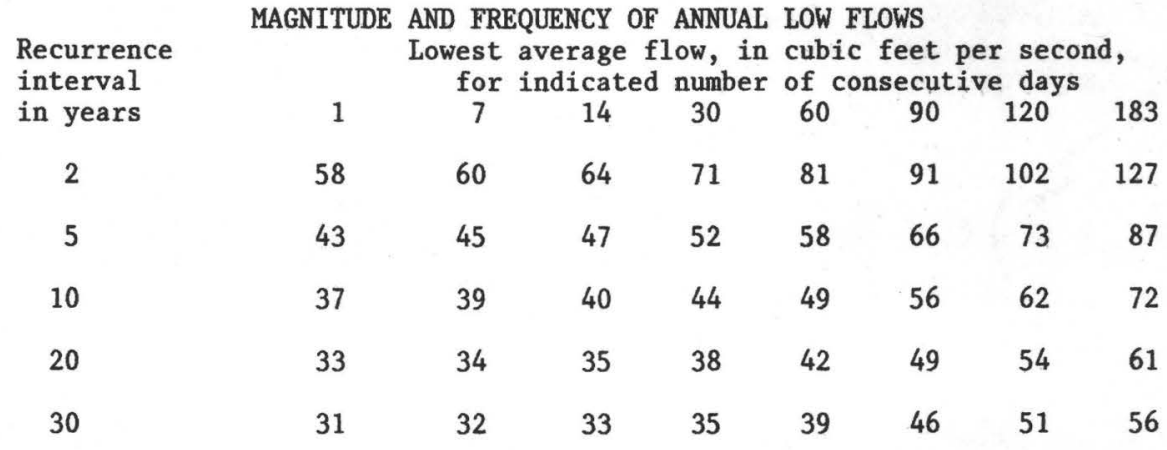

02367310 Juniper Creek at State Highway 85, near Niceville, Fla.

LOCATION.--Lat $30^{\circ} 33^{\prime} 26^{\prime \prime}$, long $86^{\circ} 31^{\prime} 11^{\prime \prime}$, in NW $\frac{3}{4}$ sec.26, T.1 N., R.23 W., Okaloosa County, Hydrologic Unit 03140102 , on left bank $20 \mathrm{ft}$ downstream from southbound bridge on State Highway $85,0.8 \mathrm{mi}$ upstream from mouth at Turkey Creek, and 3.0 mi northwest of Niceville. Re-established, May 11, 1977.

DRAINAGE AREA. $--27.6 \mathrm{mi}^{2}$.

PERIOD OF RECORD ANALYZED.--April 1966 to March 1975.

REMARKS.--Data adjusted by use of a flow correlation analysis with gaging station 02367000, Alaqua Creek near DeFuniak Springs.

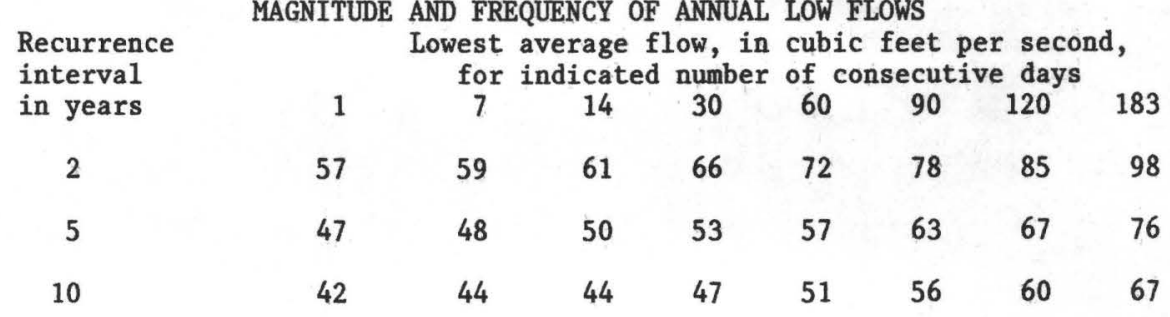




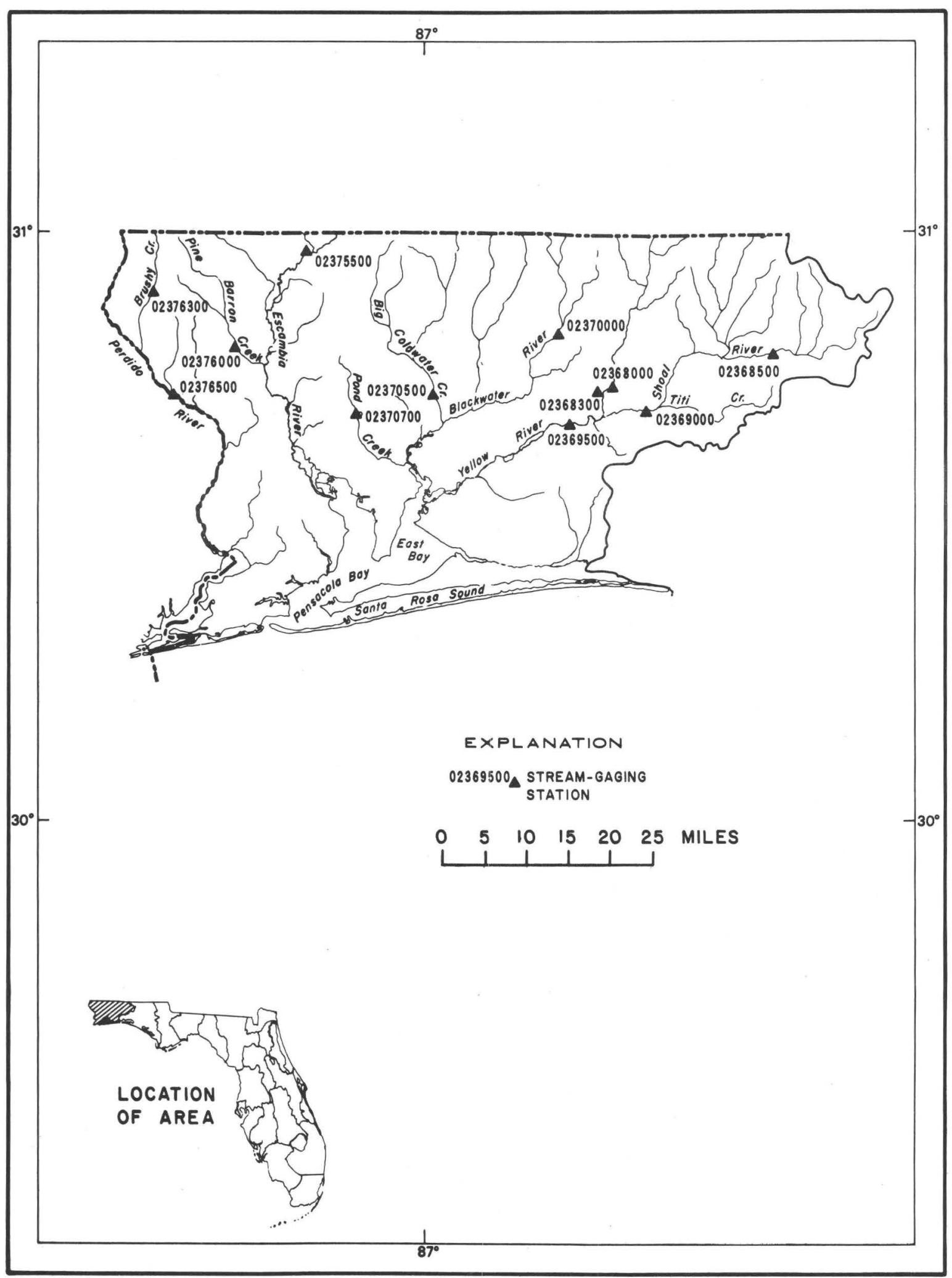

Figure 17.--Location of stream-gaging stations in the Yellow, Blackwater, Escambia and Perdido River basins. 


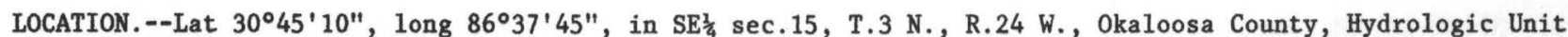
03140103, near center on downstream side of bridge of U.S. Highway 90, 0.5 mi east of Milligan, $0.5 \mathrm{mi}$ upstream from Trammel Creek, $6.7 \mathrm{mi}$ upstream from Shoal River, an 40 mi upstream from mouth.

DRAINAGE AREA. $--624 \mathrm{mi}^{2}$.

PERIOD OF RECORD ANALYZED.--April 1939 to March 1978.

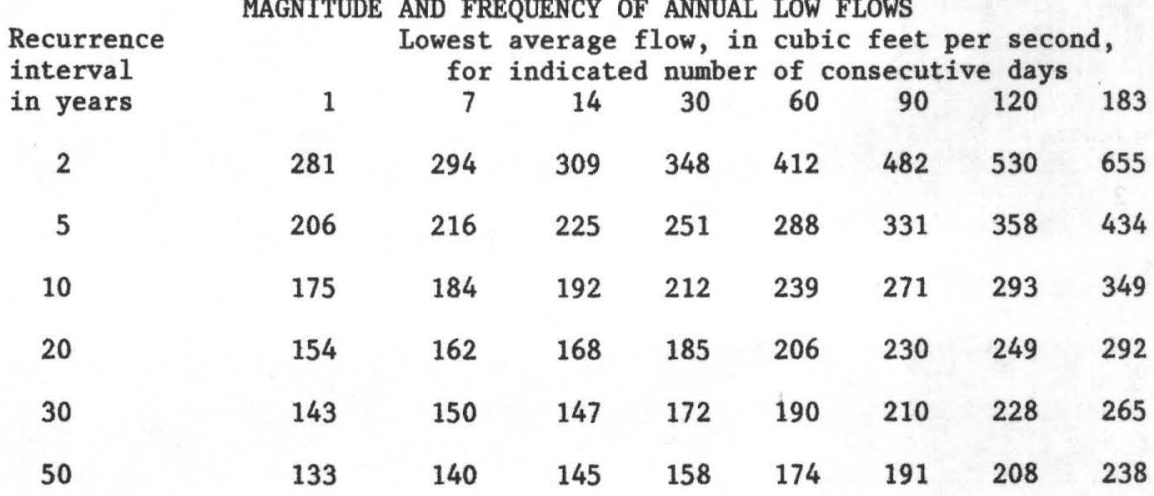

02368300 Baggett Creek near Milligan, Fla.

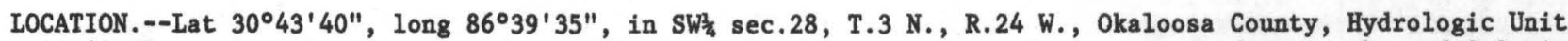
03140103 , at left downstream side of culvert on U.S. Highway $90,1.2$ mi upstream from mouth, and 2.0 mi southwest of Milligan.

DRAINAGE AREA. $--7.77 \mathrm{mi}^{2}$.

PERIOD OF RECORD ANALYZED.--April 1964 to March 1978.

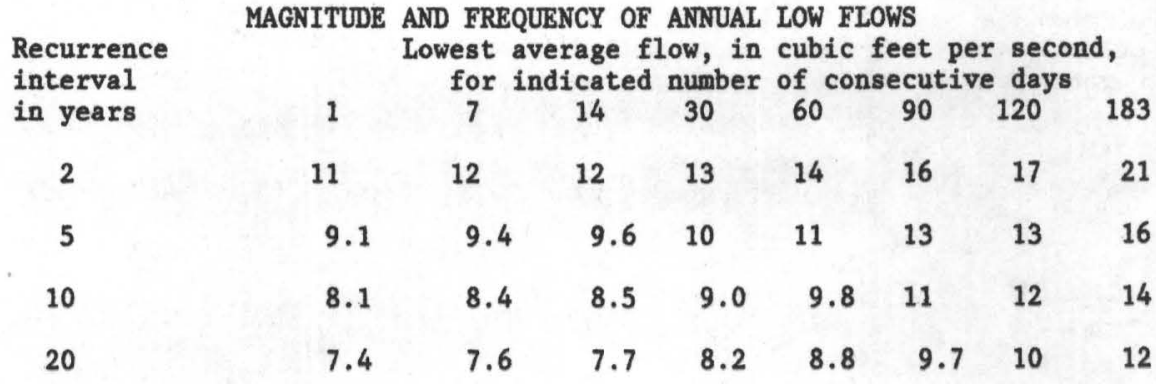


LOCATION.--Lat $30^{\circ} 47^{\prime} 45^{\prime \prime}$, long $86^{\circ} 18^{\prime} 25^{\prime \prime}$, in SW/ sec.36, T.4 N., R.21 W., Walton County, Hydrologic Unit 03140103 , near center span on downstream side of bridge on State Highway 285, about 200 ft downstream from Machine Branch, $3.9 \mathrm{mi}$ north of Mossy Head, and 34 mi upstream from mouth.

DRAINAGE AREA. --123 $\mathrm{mi}^{2}$.

PERIOD OF RECORD ANALYZED.--April 1951 to March 1978.

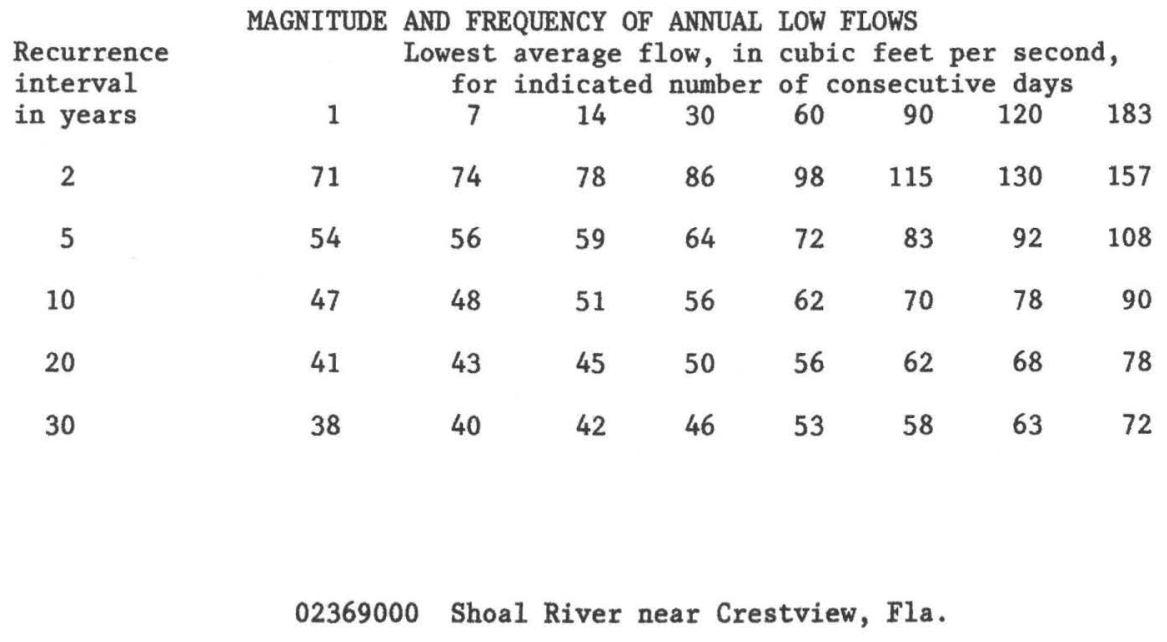

LOCATION.--Iat $30^{\circ} 41^{\prime} 50^{\prime \prime}$, long $86^{\circ} 34^{\prime} 15^{\prime \prime}$, in SW sec.5, T.2 N., R.23 W., Okaloosa County, Hydrologic Unit 03140103 , near center of bridge on downstream side of downstream bridge on State Highway $85,3.5$ mi downstream from Titi Creek, $4.2 \mathrm{mi}$ south of Crestview, and $7 \mathrm{mi}$ upstream from mouth.

DRAINAGE AREA. $--474 \mathrm{mi}^{2}$.

PERIOD OF RECORD ANALYZED.--April 1939 to March 1978.

\begin{tabular}{|c|c|c|c|c|c|c|c|c|}
\hline $\begin{array}{l}\text { Recurrence } \\
\text { interval }\end{array}$ & 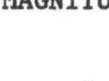 & $\begin{array}{l}\text { owest } \\
\text { for }\end{array}$ & average $f$ & $\begin{array}{l}\text { low, in } \\
\text { number }\end{array}$ & $\begin{array}{l}\text { cubi } \\
\text { of } c\end{array}$ & $\begin{array}{l}\text { feet } \\
\text { isecut }\end{array}$ & $\begin{array}{l}x \text { sec } \\
\text { re day }\end{array}$ & \\
\hline in years & 1 & 7 & 14 & 30 & 60 & 90 & $120^{\circ}$ & 183 \\
\hline 2 & 396 & 420 & 441 & 487 & 545 & 620 & 675 & 785 \\
\hline 5 & 315 & 330 & 344 & 371 & 407 & 455 & 489 & 567 \\
\hline 10 & 280 & 291 & 303 & 324 & 351 & 386 & 413 & 477 \\
\hline 20 & 255 & 263 & 273 & 289 & 313 & 337 & 360 & 413 \\
\hline 30 & 240 & 250 & 258 & 270 & 295 & 312 & 335 & 380 \\
\hline 50 & 230 & 235 & 243 & 256 & 276 & 288 & 308 & 351 \\
\hline
\end{tabular}




\section{YELLOW RIVER BASIN}

02369500 Yellow River near Holt, Fla.

LOCATION.--Lat $30^{\circ} 40^{\prime} 2^{\prime \prime}$, long $86^{\circ} 4^{\prime} 50^{\prime \prime}$, in sec.16, T.2 N., R.25 W., Okaloosa County, Hydrologic Unit 03140103, near right bank on upstream side of county highway bridge $2 \frac{1}{2}$ mi south of Holt.

DRAINAGE AREA. $--1,210 \mathrm{mi}^{2}$.

PERIOD OF RECORD ANALYZED.--April 1934 to March 1941.

Recurrence

interval

in years

2

5

10
MAGNITUDE AND FREQUENCY OF ANNUAL LOW FLOWS

Lowest average flow, in cubic feet per second, for indicated number of consecutive days

$\begin{array}{llllllll}1 & 7 & 14 & 30 & 60 & 90 & 120 & 183\end{array}$

$\begin{array}{llllllll}923 & 967 & 995 & 1040 & 1180 & 1290 & 1390 & 1690\end{array}$

$\begin{array}{llllllll}854 & 874 & 893 & 928 & 1010 & 1080 & 1160 & 1380\end{array}$

$\begin{array}{llllllll}821 & 836 & 853 & 881 & 928 & 980 & 1390 & 1250\end{array}$




\section{BLACKWATER RIVER BASIN}

02370000 Blackwater River near Baker, Fla.

LOCATION.--Lat $30^{\circ} 50^{\prime} 00^{\prime \prime}$, long $86^{\circ} 4^{\prime} 05^{\prime \prime}$, in SW sec.22, T.4 N., R.25 W., Okaloosa County, Blackwater River State Forest, Hydrologic Unit 03140104, near right bank on downstream side of bridge on State Highway 4, $0.3 \mathrm{mi}$ downstream from Red Wash Branch, $3.8 \mathrm{mi}$ northwest of Baker, and $35 \mathrm{mi}$ upstream from mouth.

DRAINAGE AREA. $--205 \mathrm{mi}^{2}$.

PERIOD OF RECORD ANALYZED.--April 1950 to March 1978.

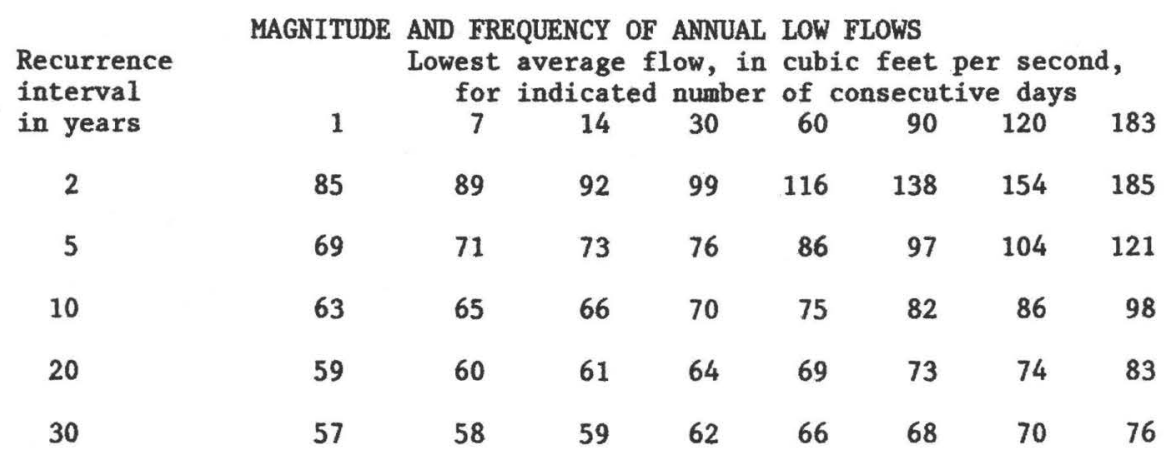

02370200 Big Juniper Creek near Munson, F1a.

LOCATION.--Lat $30^{\circ} 51^{\prime} 50^{\prime \prime}$, long $86^{\circ} 54^{\prime} 20^{\prime \prime}$, in SW sec.12, T.4 N., R.27 W., Santa Rosa County, Hydrologic Unit 03140104 , on right bank $300 \mathrm{ft}$ upstream from bridge on State Highway 4, 0.3 mi downstream from Gunstock Branch, $2 \mathrm{mi}$ west of Munson, and $3.7 \mathrm{mi}$ upstream from Sweetwater Creek.

DRAINAGE AREA. $--36 \mathrm{mi}^{2}$, approximately.

PERIOD OF RECORD ANALYZED.--April 1958 to March 1966.

REMARKS.--Data adjusted by use of a flow correlation analysis with gaging station 02370000, Blackwater River near Baker.

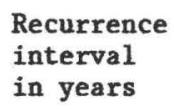

2

5

10

MAGNITUDE AND FREQUENCY OF ANNUAL LOW FLOWS

Lowest average flow, in cubic feet per second, for indicated number of consecutive days

$\begin{array}{llllllll}1 & 7 & 14 & 30 & 60 & 90 & 120 & 183\end{array}$

19

$20 \quad 20$

22

$24 \quad 28$

30

35

16

17

17

18

19

$21 \quad 23$

25

15

16

16

17

18

$19 \quad 19$

22 
LOCATION.--Lat $30^{\circ} 42^{\prime} 30^{\prime \prime}$, long $86^{\circ} 58^{\prime} 20^{\prime \prime}$, in SW/ sec.5, T.2 N., R. 27 W., Santa Rosa County, Hydrologic Unit 03140104 , at right bank on downstream side of bridge on State Highway 191, 3 mi upstream from mouth, and $6.5 \mathrm{mi}$ northeast of Milton.

DRAINAGE AREA. --237 $\mathrm{mi}^{2}$.

PERIOD OF RECORD ANALYZED.--April 1939 to March 1978.

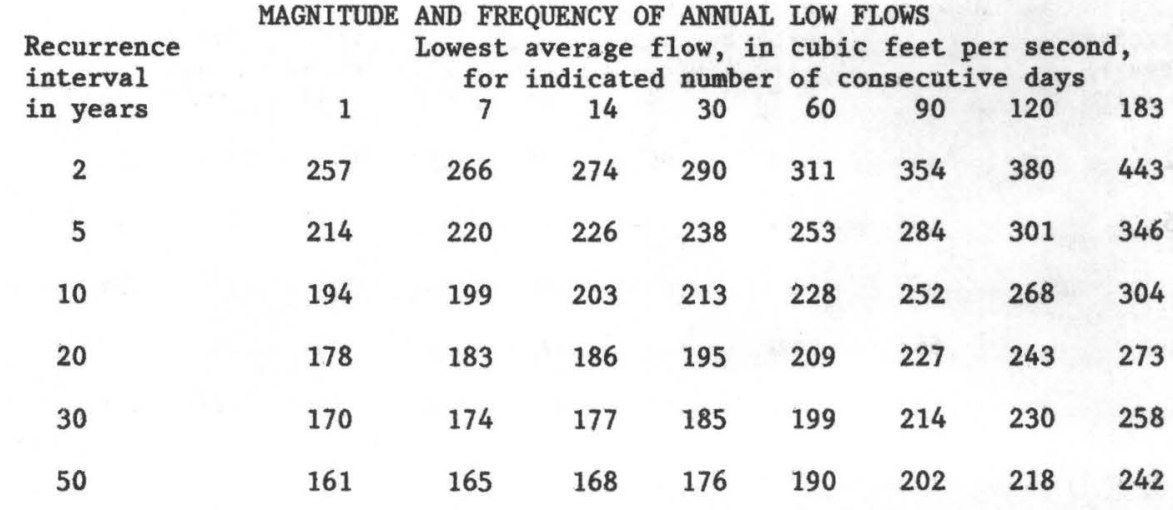

02370700 Pond Creek near Milton, Fla.

LOCATION.--Lat $30^{\circ} 40^{\prime} 50^{\prime \prime}$, long $87^{\circ} 07^{\prime} 55^{\prime \prime}$, in SE $\frac{1}{4}$ sec.15, T.2 N., R.29 W., Santa Rosa County, Hydrologic Unit 03140104 , near center of span on downstream side of bridge on State Highway $191,0.6 \mathrm{mi}$ downstream from Reader Creek, $6.4 \mathrm{mi}$ northwest of Milton, and $10 \mathrm{mi}$ upstream from mouth.

DRAINAGE AREA. $--58.7 \mathrm{mi}^{2}$.

PERIOD OF RECORD ANALYZED.--April 1958 to March 1978.

Recurrence
interval
in years
2
5
10
20

MAGNITUDE AND FREQUENCY OF ANNUAL LOW FLOWS Lowest average flow, in cubic feet per second, $\begin{array}{ccccccc}\text { for indicated number } & \text { of consecutive days } & \\ 7 & 14 & 30 & 60 & 90 & 120 & 183\end{array}$

1

47

$\begin{array}{lllllll}48 & 49 & 51 & 56 & 60 & 63 & 67\end{array}$

38

39

40

34

30

34

$\begin{array}{lllllll}34 & 35 & 36 & 38 & 40 & 42 & 44 \\ 30 & 31 & 32 & 33 & 35 & 36 & 38\end{array}$

30

31

41

44

47

$49 \quad 51$

30




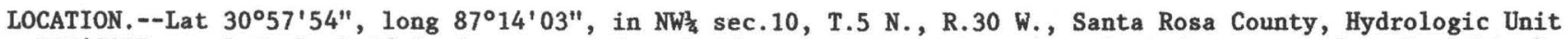
03140305, on left bank $16 \mathrm{ft}$ downstream from bridge on State Highway 4, $1.2 \mathrm{mi}$ downstream from Escambia Creek, $1.7 \mathrm{mi}$ east of Century, and $52 \mathrm{mi}$ upstream from mouth.

DRAINAGE AREA.--3,817 $\mathrm{mi}^{2}$.

PERIOD OF RECORD ANALYZED.--April 1935 to March 1978.

\begin{tabular}{|c|c|c|c|c|c|c|c|c|}
\hline \multirow{2}{*}{$\begin{array}{l}\text { Recurrence } \\
\text { interval } \\
\text { in years }\end{array}$} & MAGNITUD & $\begin{array}{l}\text { AND FT } \\
\text { Lowest } \\
\text { for }\end{array}$ & $\begin{array}{l}\text { UENCY } \\
\text { verag } \\
\text { ndica }\end{array}$ & $\begin{array}{l}\text { E ANNL } \\
\text { flow, } \\
\text { numb }\end{array}$ & $\begin{array}{l}\text { LOW } \\
\text { cubi } \\
\text { of }\end{array}$ & $\begin{array}{l}\text { LoWS } \\
\text { feet } \\
\text { asecut }\end{array}$ & $\begin{array}{l}\text { er sec } \\
\text { ve day }\end{array}$ & nd, \\
\hline & 1 & 7 & 14 & 30 & 60 & 90 & 120 & 183 \\
\hline 2 & 1100 & 1190 & 1240 & 1380 & 1630 & 1940 & 2160 & 2680 \\
\hline 5 & 836 & 889 & 917 & 1000 & 1130 & 1330 & 1480 & 1830 \\
\hline 10 & 735 & 777 & 794 & 859 & 947 & 1100 & 1240 & 1530 \\
\hline 20 & 666 & 701 & 710 & 761 & 826 & 949 & 1080 & 1330 \\
\hline 30 & 630 & 665 & 675 & 710 & 770 & 860 & 1000 & 1210 \\
\hline 50 & 601 & 630 & 631 & 669 & 715 & 803 & 938 & 1140 \\
\hline
\end{tabular}

02376000 Pine Barren Creek near Barth, Fla.

LOCATION.--Lat $30^{\circ} 47^{\prime} 55^{\prime \prime}$, long $87^{\circ} 22^{\prime} 05^{\prime \prime}$, in SW sec.5, T.3 N., R.31 W., Escambia County, Hydrologic Unit 03140305 , near right bank $10 \mathrm{ft}$ downstream from Wiggins Bridge on private road, $0.3 \mathrm{mi}$ upstream from Blue Water Creek, $4.0 \mathrm{mi}$ northwest of Barth, and $7.3 \mathrm{mi}$ upstream from mouth.

DRAINAGE AREA. $--75.3 \mathrm{mi}^{2}$.

PERIOD OF RECORD ANALYZED.--April 1953 to March 1978.

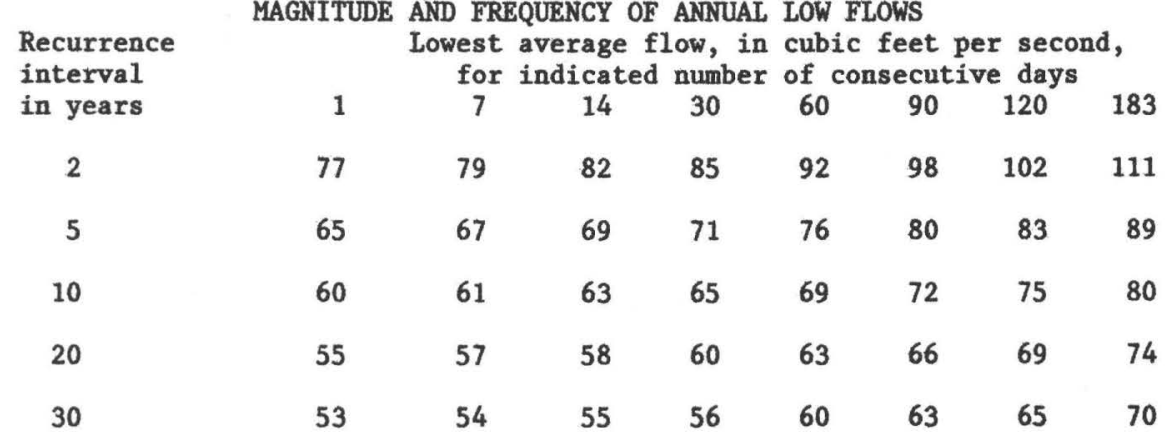


LOCATION.--Lat $30^{\circ} 53^{\prime} 21^{\prime \prime}$, long $87^{\circ} 32^{\prime} 24^{\prime \prime}$, in SE $\frac{3}{4}$ sec.4, T.4 N., R.33 W., Escambia County, Hydrologic Unit 03140106, near right bank on downstream side of county road bridge, 1,000 ft downstream from Rocky Creek, $2.0 \mathrm{mi}$ west of Walnut Hill, and $7.9 \mathrm{mi}$ upstream from mouth.

DRAINAGE AREA. --49 $\mathrm{mi}^{2}$, approximately.

PERIOD OF RECORD ANALYZED.--April 1958 to March 1977.

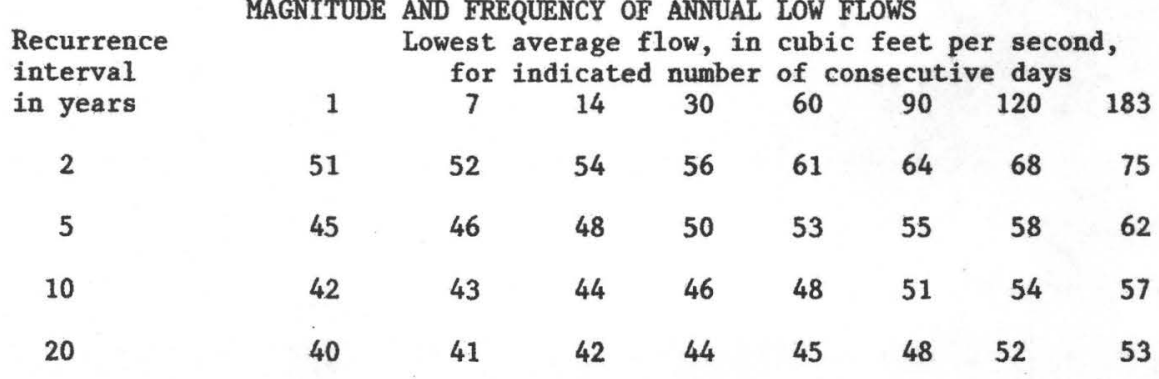

02376500 Perdido River at Barrineau Park, Fla.

LOCATION.--Lat $30^{\circ} 41^{\prime} 25^{\prime \prime}$, long $87^{\circ} 26^{\prime} 25^{\prime \prime}$, in NW/ sec.23, T.4 S., R.6 E., Baldwin County, Ala., Hydrologic Unit

03140106 , on right bank $25 \mathrm{ft}$ downstream from county highway bridge, 1,000 ft downstream from Alligator Creek, 0.5 mi southwest of Barrineau Park, and 27 mi upstream from mouth.

DRAINAGE AREA. $--394 \mathrm{mi}^{2}$.

PERIOD OF RECORD ANALYZED.--April 1942 to March 1977.

\begin{tabular}{|c|c|c|c|c|c|c|c|c|}
\hline \multirow{2}{*}{$\begin{array}{l}\text { Recurrence } \\
\text { interval } \\
\text { in years }\end{array}$} & MAGNITUL & $\begin{array}{l}\mathrm{ND} F \\
\text { owest } \\
\text { for }\end{array}$ & $\begin{array}{l}\text { ENCY } \\
\text { erage } \\
\text { dicat }\end{array}$ & $\begin{array}{l}\text { ANN } \\
\text { Low, } \\
\text { numb }\end{array}$ & $\begin{array}{l}\text { LOW } \\
\text { cub } \\
\text { of }\end{array}$ & $\begin{array}{l}\text { OWS } \\
\text { feet } \\
\text { secut }\end{array}$ & \multicolumn{2}{|c|}{$\begin{array}{l}\text { second, } \\
\text { e days }\end{array}$} \\
\hline & 1 & 7 & 14 & 30 & 60 & 90 & 120 & 183 \\
\hline 2 & 270 & 283 & 294 & 319 & 364 & 413 & 457 & 519 \\
\hline 5 & 230 & 241 & 247 & 261 & 288 & 319 & 344 & 39 \\
\hline 10 & 210 & 221 & 226 & 234 & 253 & 276 & 295 & 336 \\
\hline 20 & 200 & 205 & 208 & 214 & 227 & 244 & 260 & 296 \\
\hline 30 & 190 & 195 & 199 & 201 & 210 & 225 & 240 & 270 \\
\hline 50 & 185 & 189 & 190 & 193 & 199 & 211 & 225 & 256 \\
\hline
\end{tabular}


UNITED STATES DEPARTMENT OF THE INTERIOR GEOLOGICAL SURVEY

325 John Knox Rd--Suite F240

Tallahassee, Florida 32303
POSTAGE AND FEES PAID

U.S. DEPARTMENT OF THE INTERIOR INT. 413 